\title{
Asymmetric conjugate addition of ethylene sulfonyl fluorides to 3-amido-2-oxindoles: synthesis of chiral spirocyclic oxindole sultams
}

\author{
Jie Chen, Bao-qin Huang, Zeng-qing Wang, Xue-jing Zhang, Ming Yan* \\ Guangdong Provincial Key Laboratory of Chiral Molecules and Drug Discovery, School of \\ Pharmaceutical Sciences, Sun Yat-sen University, Guangzhou 510006, China. \\ E-mail: yanming@mail.sysu.edu.cn
}

Supporting Information

Table of Contents

\begin{tabular}{|c|c|}
\hline 1. General information............ & $\mathrm{S} 2$ \\
\hline 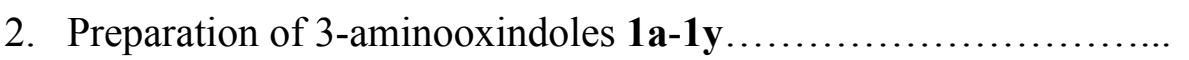 & $\mathrm{S} 2$ \\
\hline 3. Typical procedures and characterization data for products $\mathbf{3 a - 3 y} \ldots$ & $\mathrm{S} 13$ \\
\hline 4. Typical procedures and characterization data for products $5 \ldots \ldots$. & S45 \\
\hline 5. Typical procedures and characterization data for products 6ab-6ai & S49 \\
\hline 6. Procedure for the gram-scale synthesis of $\mathbf{3 a} \ldots$. & S59 \\
\hline 7. Procedures for the transformations of $\mathbf{3 a} \ldots \ldots \ldots \ldots \ldots \ldots \ldots \ldots \ldots \ldots \ldots$ & S60 \\
\hline 8. The X-ray crystal data of products $3 \mathbf{v}$ and $\mathbf{6 a b} \ldots \ldots \ldots \ldots \ldots \ldots$ & S62 \\
\hline 9. References............... & S64 \\
\hline 10. NMR spectra... & S65 \\
\hline
\end{tabular}




\section{General information}

${ }^{1} \mathrm{H}$ NMR and ${ }^{13} \mathrm{C}$ NMR spectra were recorded on Bruker $400 \mathrm{MHz}$ or $500 \mathrm{MHz}$ spectrometer in $\mathrm{CDCl}_{3}$ or DMSO- $d_{6}$ with tetramethylsilane (TMS) as the internal standard. Melting points were measured on a WRS-2A melting point apparatus and are uncorrected. Chemical shifts of protons are reported in parts per million downfield from tetramethylsilane and are referenced to residual protium in the NMR solvent $\left(\mathrm{CDCl}_{3}: \delta 7.26\right.$; DMSO- $\left.d_{6}: \delta 2.50\right)$. Chemical shifts of carbon are referenced to the carbon resonances of the solvent $\left(\mathrm{CDCl}_{3}: \delta 77.0 ; \mathrm{DMSO}-d_{6}: \delta 39.5\right)$. Peaks are labeled as singlet (s), broad singlet (br), doublet (d), triplet (t), double doublet (dd), multiplet (m). The enantiomeric excesses were determined by HPLC with Daicel CHIRALCEL, CHIRALPAK and Nu-Analytical HPLC columns. The chiral HPLC methods were calibrated with the corresponding racemic mixtures. All products were further characterized by HRMS (high resolution mass spectra). Copies of their ${ }^{1} \mathrm{H}$ NMR and ${ }^{13} \mathrm{C}$ NMR spectra were provided.

All reagents were used as received from commercial suppliers without further purification unless otherwise noted. All solvents were dried and distilled prior to use according to the standard protocols. $N$-Bn-isatin oximes ${ }^{1}$, ethenesulfonyl fluoride ${ }^{2}, \beta$-arylethenesulfonyl fluorides ${ }^{3-4}$ and organocatalysts $\mathbf{4 b}-\mathbf{4} \mathbf{i}^{5-9}$ were synthesized in accordance with the reported procedures.

\section{Preparation of 3-amidooxindoles (1a-1y)}

\subsection{Typical procedures for the synthesis of substrates $1 \mathrm{a}-1 \mathrm{q}$}

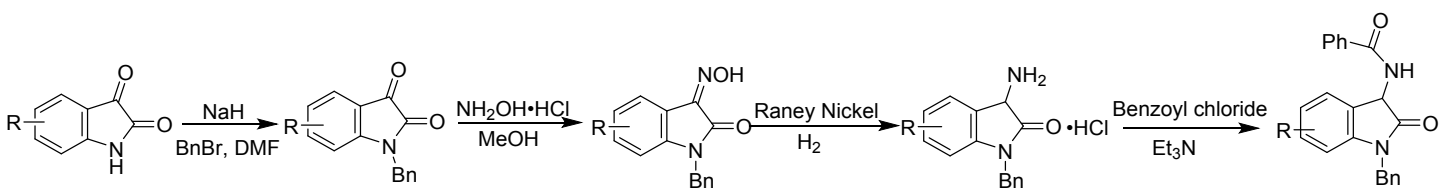

A solution of isatin $(11 \mathrm{mmol})$ in DMF $(30 \mathrm{~mL})$ was cooled under an ice bath. $\mathrm{NaH}(60 \%$ dispersion in mineral oil, $12 \mathrm{mmol}$ ) was added portionwise. The color of solution changed from orange to deep purple. When the gas evolution stopped, benzyl bromide $(13 \mathrm{mmol})$ was added slowly, whereupon the mixture was turned red-brown. After the reaction mixture was stirred for $15 \mathrm{~min}$ at room temperature, $\mathrm{H}_{2} \mathrm{O}(100 \mathrm{~mL})$ was added to precipitate $N$-Bn-isatin.

To a solution of $\mathrm{N}$-Bn isatin $(5 \mathrm{mmol})$ in $\mathrm{MeOH}(100 \mathrm{~mL})$ was added hydroxylamine hydrochloride $(6.0 \mathrm{mmol})$ and potassium carbonate $(6.0 \mathrm{mmol})$. The resulting mixture was stirred under reflux overnight. The reaction mixture was concentrated under reduced pressure, and the residue was purified by column chromatography on silica gel (pentane/EtOAc $=2: 1$ ) to give isatin oxime as a yellow solid. 
To a suspension of isatin oxime $(5 \mathrm{mmol})$ in $\mathrm{MeOH}(200 \mathrm{~mL})$ was added Raney Nickel at room temperature in $100 \mathrm{~mL}$ high pressure reactor. The reaction mixture was stirred under $\mathrm{H}_{2}$ atmosphere $(8$ atm) for $10 \mathrm{~h}$. Then, concentrated $\mathrm{HCl}(20 \mathrm{~mL})$ was added to the mixture. After Raney Nickel was removed by the filtration, the filtrate was concentrated under reduced pressure. The crude product was purified by washing with dichloromethane $(100 \mathrm{~mL})$ to provide 3 -amino-oxindole hydrochloride as a white solid.

To a solution of 3-amino-oxindole hydrochloride $(2.5 \mathrm{mmol})$ in $\mathrm{CH}_{2} \mathrm{Cl}_{2}(20 \mathrm{~mL})$ was added $\mathrm{Et}_{3} \mathrm{~N}$ $(0.55 \mathrm{~mL}, 3.75 \mathrm{mmol})$ at $0{ }^{\circ} \mathrm{C}$ under $\mathrm{N}_{2}$ atmosphere. The resulting mixture was vigorously stirred for $15 \mathrm{~min}$, and then benzoyl chloride $(2.75 \mathrm{mmol})$ was added slowly via a syringe. The mixture was stirred for $1 \mathrm{~h}$ and concentrated. The residue was purified by flash chromatography to furnish the product as a white solid.

\subsection{Typical procedures for the synthesis of substrates $1 \mathrm{r}-1 \mathrm{y}$}<smiles>[R]C(=O)NC1C(=O)N(Cc2ccccc2)c2ccccc21</smiles>

To a solution of 3-amino-oxindole hydrochloride $(2.5 \mathrm{mmol})$ in $\mathrm{CH}_{2} \mathrm{Cl}_{2}(20 \mathrm{~mL})$ was added $\mathrm{Et}_{3} \mathrm{~N}(0.55$ $\mathrm{mL}, 3.75 \mathrm{mmol}$ ) at $0{ }^{\circ} \mathrm{C}$ under $\mathrm{N}_{2}$ atmosphere. The resulting mixture was vigorously stirred for $15 \mathrm{~min}$, and then acyl chloride $(2.75 \mathrm{mmol})$ was added slowly via a syringe. The mixture was stirred for $1 \mathrm{~h}$ and concentrated. The residue was purified by flash chromatography to furnish the product as a white solid.

\subsection{Characterizations of substrates 1a-1y}

\section{$N$-(1-Benzyl-2-oxoindolin-3-yl)benzamide (1a)}<smiles>O=C(NC1C(=O)N(Cc2ccccc2)c2ccccc21)c1ccccc1</smiles>

This compound was prepared according to typical procedure 2.1. Petroleum ether/ethyl acetate $=5: 1$ was used as the eluent for column chromatography. White solid (684 mg, 80\%); m.p. 199.7-200.6 ${ }^{\circ} \mathrm{C}$; ${ }^{1} \mathbf{H}$ NMR $\left(500 \mathrm{MHz}, \mathrm{CDCl}_{3}\right) \delta 7.81(\mathrm{~d}, J=7.5 \mathrm{~Hz}, 2 \mathrm{H}), 7.46(\mathrm{t}, J=7.5 \mathrm{~Hz}, 1 \mathrm{H}), 7.42-7.34(\mathrm{~m}, 6 \mathrm{H}), 7.34-$ $7.30(\mathrm{~m}, 2 \mathrm{H}), 7.28-7.23(\mathrm{~m}, 1 \mathrm{H}), 7.18(\mathrm{t}, J=7.5 \mathrm{~Hz}, 1 \mathrm{H}), 7.01(\mathrm{t}, J=7.5 \mathrm{~Hz}, 1 \mathrm{H}), 6.73(\mathrm{~d}, J=8.0 \mathrm{~Hz}$, 
$1 \mathrm{H}), 5.47(\mathrm{~d}, J=7.0 \mathrm{~Hz}, 1 \mathrm{H}), 5.00(\mathrm{~d}, J=15.5 \mathrm{~Hz}, 1 \mathrm{H}), 4.87(\mathrm{~d}, J=15.5 \mathrm{~Hz}, 1 \mathrm{H}) .{ }^{13} \mathbf{C}$ NMR $(126 \mathrm{MHz}$, $\left.\mathrm{CDCl}_{3}\right) \delta 174.72,167.60,142.95,135.47,133.06,131.92,129.11,128.87,128.53,127.74,127.36$, 126.61, 124.55, 123.13, 109.41, 53.33, 44.22. HRMS (ESI) $m / z$ : Calcd. for $\mathrm{C}_{22} \mathrm{H}_{19} \mathrm{~N}_{2} \mathrm{O}_{2},[\mathrm{M}+\mathrm{H}]^{+}$, 341.1269; found: 341.1243 .

$N$-(2-Oxo-1-phenylindolin-3-yl)benzamide (1b)<smiles>O=C(NC1C(=O)N(c2ccccc2)c2ccccc21)c1ccccc1</smiles>

This compound was prepared according to typical procedure 2.1. Petroleum ether/ethyl acetate $=5: 1$ was used as the eluent for column chromatography. White solid (680 mg, 83\%); m.p. 204.5-206.6 ${ }^{\circ} \mathrm{C}$; ${ }^{1}$ H NMR $\left(400 \mathrm{MHz}\right.$, DMSO- $\left.d_{6}\right) \delta 9.57(\mathrm{~d}, J=7.6 \mathrm{~Hz}, 1 \mathrm{H}), 7.93(\mathrm{~d}, J=7.2 \mathrm{~Hz}, 2 \mathrm{H}), 7.63-7.56(\mathrm{~m}, 3 \mathrm{H})$, 7.54-7.46 (m, 5H), $7.32(\mathrm{~d}, J=7.2 \mathrm{~Hz}, 1 \mathrm{H}), 7.26(\mathrm{t}, J=7.6 \mathrm{~Hz}, 1 \mathrm{H}), 7.08(\mathrm{t}, J=7.2 \mathrm{~Hz}, 1 \mathrm{H}), 6.76(\mathrm{~d}, J$ $=8.0 \mathrm{~Hz}, 1 \mathrm{H}), 5.46(\mathrm{~d}, J=7.6 \mathrm{~Hz}, 1 \mathrm{H}) .{ }^{13} \mathbf{C}$ NMR $\left(101 \mathrm{MHz}, \mathrm{DMSO}-d_{6}\right) \delta 173.94,166.48,144.49$, 135.23, 133.62, 132.25, 130.07, 129.01, 128.96, 128.42, 127.87, 127.67, 127.15, 124.21, 123.18, 109.19, 53.18. HRMS (ESI) $m / z$ : Calcd. for $\mathrm{C}_{21} \mathrm{H}_{17} \mathrm{~N}_{2} \mathrm{O}_{2},[\mathrm{M}+\mathrm{H}]^{+}, 329.1285$; found: 329.1274 .

$N$-(1-Methyl-2-oxoindolin-3-yl)benzamide (1c)<smiles>CN1C(=O)C(NC(=O)c2ccccc2)c2ccccc21</smiles>

This compound was prepared according to typical procedure 2.1. Petroleum ether/ethyl acetate $=5: 1$ was used as the eluent for column chromatography. White solid (532 mg, 80\%); m.p. 190.5-192.3 ${ }^{\circ} \mathrm{C}$; ${ }^{1} \mathbf{H}$ NMR $\left(500 \mathrm{MHz}, \mathrm{CDCl}_{3}\right) \delta 7.81(\mathrm{~d}, J=7.5 \mathrm{~Hz}, 2 \mathrm{H}), 7.51-7.45(\mathrm{~m}, 1 \mathrm{H}), 7.43-7.36(\mathrm{~m}, 3 \mathrm{H}), 7.35-7.30$ (m, 1H), $7.15(\mathrm{~d}, J=7.0 \mathrm{~Hz}, 1 \mathrm{H}), 7.06(\mathrm{t}, J=7.5 \mathrm{~Hz}, 1 \mathrm{H}), 6.86(\mathrm{~d}, J=8.0 \mathrm{~Hz}, 1 \mathrm{H}), 5.49$ (d, $J=7.0 \mathrm{~Hz}$, 1H), 3.23 (s, 3H). ${ }^{13} \mathbf{C}$ NMR (101 MHz, $\left.\mathrm{CDCl}_{3}\right) \delta 174.54,167.69,143.77,133.09,131.93,129.27,128.54$, 127.28, 126.65, 124.68, 123.16, 108.36, 53.14, 26.56. HRMS (ESI) $m / z$ : Calcd. for $\mathrm{C}_{16} \mathrm{H}_{15} \mathrm{~N}_{2} \mathrm{O}_{2},[\mathrm{M}+\mathrm{H}]^{+}$, 267.1128; found: 267.1127 .

\section{$N$-(1-Benzyl-5-fluoro-2-oxoindolin-3-yl)benzamide (1d)}<smiles>O=C(NC1C(=O)N(c2ccccc2)c2ccc(F)cc21)c1ccccc1</smiles> 
This compound was prepared according to typical procedure 2.1 . Petroleum ether/ethyl acetate $=5: 1$ was used as the eluent for column chromatography. White solid (720 mg, 80\%); m.p.198.9-201.3 ${ }^{\circ} \mathrm{C} ;{ }^{1} \mathbf{H}$ NMR $\left(400 \mathrm{MHz}, \mathrm{CDCl}_{3}\right) \delta 7.82(\mathrm{~d}, J=7.6 \mathrm{~Hz}, 2 \mathrm{H}), 7.54-7.49(\mathrm{~m}, 1 \mathrm{H}), 7.45-7.28(\mathrm{~m}, 7 \mathrm{H}), 7.25-7.12$ $(\mathrm{m}, 2 \mathrm{H}), 6.89(\mathrm{td}, J=9.2,2.4 \mathrm{~Hz}, 1 \mathrm{H}), 6.65(\mathrm{dd}, J=8.8,4.0 \mathrm{~Hz}, 1 \mathrm{H}), 5.43(\mathrm{~d}, J=3.6 \mathrm{~Hz}, 1 \mathrm{H}), 5.00(\mathrm{~d}$, $J=15.6 \mathrm{~Hz}, 1 \mathrm{H}), 4.92(\mathrm{~d}, J=15.6 \mathrm{~Hz}, 1 \mathrm{H}) .{ }^{13} \mathbf{C} \mathbf{N M R}\left(101 \mathrm{MHz}, \mathrm{CDCl}_{3}\right) \delta 174.27,167.65,159.46(\mathrm{~d}$, $\left.J_{\mathrm{C}-\mathrm{F}}=241.8 \mathrm{~Hz}\right), 138.81,135.13,132.80,132.16,128.95,128.65,128.13\left(\mathrm{~d}, J_{\mathrm{C}-\mathrm{F}}=8.4 \mathrm{~Hz}\right), 127.87$, $127.35,127.31,115.41\left(\mathrm{~d}, J_{\mathrm{C}-\mathrm{F}}=23.6 \mathrm{~Hz}\right), 112.85\left(\mathrm{~d}, J_{\mathrm{C}-\mathrm{F}}=25.2 \mathrm{~Hz}\right), 110.04\left(\mathrm{~d}, J_{\mathrm{C}-\mathrm{F}}=8.0 \mathrm{~Hz}\right), 53.62$, 44.42. ${ }^{19} \mathbf{F}$ NMR (471 MHz, $\left.\mathrm{CDCl}_{3}\right) \delta$-119.64. HRMS (ESI) $m / z$ : Calcd. for $\mathrm{C}_{22} \mathrm{H}_{18} \mathrm{~N}_{2} \mathrm{O}_{2} \mathrm{~F},[\mathrm{M}+\mathrm{H}]^{+}$, 361.1347; found: 361.1336 .

\section{$N$-(1-Benzyl-5-chloro-2-oxoindolin-3-yl)benzamide (1e)}<smiles>O=C(NC1C(=O)N(Cc2ccccc2)c2ccc(Cl)cc21)c1ccccc1</smiles>

This compound was prepared according to typical procedure 2.1. Petroleum ether/ethyl acetate $=5: 1$ was used as the eluent for column chromatography. White solid (760 mg, 81\%); m.p. 225.4-227.5 ${ }^{\circ} \mathrm{C}$; ${ }^{1} \mathbf{H}$ NMR (500 MHz, DMSO-d $d_{6} \delta 9.57$ (d, $\left.J=7.5 \mathrm{~Hz}, 1 \mathrm{H}\right), 7.97-7.89$ (m, 2H), 7.61-7.56 (m, 1H), 7.51 $(\mathrm{t}, J=7.5 \mathrm{~Hz}, 2 \mathrm{H}), 7.45(\mathrm{~d}, J=7.5 \mathrm{~Hz}, 2 \mathrm{H}), 7.35(\mathrm{t}, J=7.5 \mathrm{~Hz}, 2 \mathrm{H}), 7.31-7.23(\mathrm{~m}, 3 \mathrm{H}), 6.81(\mathrm{~d}, J=8.0$ $\mathrm{Hz}, 1 \mathrm{H}), 5.39$ (d, $J=7.5 \mathrm{~Hz}, 1 \mathrm{H}), 5.00(\mathrm{~d}, J=16.0 \mathrm{~Hz}, 1 \mathrm{H}), 4.91(\mathrm{~d}, J=16.0 \mathrm{~Hz}, 1 \mathrm{H}) .{ }^{13} \mathbf{C} \mathbf{N M R}(126$ MHz, DMSO- $\left.d_{6}\right) \delta 174.21,166.49,142.43,136.41,133.50,132.28,130.02,129.00,128.93,128.50$, 127.96, 127.78, 127.67, 126.78, 123.71, 110.79, 53.29, 43.41. HRMS (ESI) m/z: Calcd. for $\mathrm{C}_{22} \mathrm{H}_{18} \mathrm{~N}_{2} \mathrm{O}_{2} \mathrm{Cl},[\mathrm{M}+\mathrm{H}]^{+}, 377.1051$; found: 377.1048 .

$N$-(1-Benzyl-5-bromo-2-oxoindolin-3-yl)benzamide (1f)<smiles>O=C(NC1C(=O)N(Cc2ccccc2)c2ccc(Br)cc21)c1ccccc1</smiles>

This compound was prepared according to typical procedure 2.1. Petroleum ether/ethyl acetate $=5: 1$ was used as the eluent for column chromatography. White solid $(840 \mathrm{mg}, 80 \%)$; m.p. $226.4-228.2^{\circ} \mathrm{C}$; ${ }^{1}$ H NMR $\left(400 \mathrm{MHz}, \mathrm{DMSO}-d_{6}\right) \delta 9.56(\mathrm{~d}, J=7.6 \mathrm{~Hz}, 1 \mathrm{H}), 7.96-7.89$ (m, 2H), 7.62-7.56 (m, 1H), 7.54$7.48(\mathrm{~m}, 2 \mathrm{H}), 7.47-7.42(\mathrm{~m}, 2 \mathrm{H}), 7.41-7.32(\mathrm{~m}, 4 \mathrm{H}), 7.30-7.25(\mathrm{~m}, 1 \mathrm{H}), 6.80-6.74(\mathrm{~m}, 1 \mathrm{H}), 5.39(\mathrm{~d}, J=$ $7.2 \mathrm{~Hz}, 1 \mathrm{H}), 4.99(\mathrm{~d}, J=16.4 \mathrm{~Hz}, 1 \mathrm{H}), 4.90(\mathrm{~d}, J=16.4 \mathrm{~Hz}, 1 \mathrm{H}) .{ }^{13} \mathbf{C}$ NMR $\left(101 \mathrm{MHz}, \mathrm{DMSO}-d_{6}\right) \delta$ 174.10, 166.49, 142.84, 136.38, 133.49, 132.29, 131.37, 130.39, 129.00, 128.94, 127.97, 127.78, 127.66, 126.37, 114.47, 111.34, 53.22, 43.38. HRMS (ESI) $m / z$ : Calcd. for $\mathrm{C}_{22} \mathrm{H}_{18} \mathrm{~N}_{2} \mathrm{O}_{2} \mathrm{Br},[\mathrm{M}+\mathrm{H}]^{+}, 421.0546$; found: 421.0545 .

N-(1-Benzyl-5-methyl-2-oxoindolin-3-yl) benzamide (1g) 
<smiles>Cc1ccc2c(c1)C(NC(=O)c1ccccc1)C(=O)N2Cc1ccccc1</smiles>

This compound was prepared according to typical procedure 2.1. Petroleum ether/ethyl acetate $=5: 1$ was used as the eluent for column chromatography. White solid (756 mg, 85\%); m.p. 204.9-206.5 ${ }^{\circ} \mathrm{C}$; ${ }^{1}$ H NMR $\left(500 \mathrm{MHz}, \mathrm{DMSO}-d_{6}\right) \delta 9.48(\mathrm{~d}, J=7.5 \mathrm{~Hz}, 1 \mathrm{H}), 7.94(\mathrm{~d}, J=7.5 \mathrm{~Hz}, 2 \mathrm{H}), 7.61-7.55(\mathrm{~m}, 1 \mathrm{H})$, $7.51(\mathrm{t}, J=7.5 \mathrm{~Hz}, 2 \mathrm{H}), 7.44$ (d, $J=7.5 \mathrm{~Hz}, 2 \mathrm{H}), 7.34$ (t, $J=7.5 \mathrm{~Hz}, 2 \mathrm{H}), 7.29-7.24(\mathrm{~m}, 1 \mathrm{H}), 7.06$ (s, $1 \mathrm{H}), 6.99$ (d, $J=8.0 \mathrm{~Hz}, 1 \mathrm{H}), 6.68(\mathrm{~d}, J=8.0 \mathrm{~Hz}, 1 \mathrm{H}), 5.39$ (d, $J=7.5 \mathrm{~Hz}, 1 \mathrm{H}) 4.96(\mathrm{~d}, J=16.0 \mathrm{~Hz}$, 1H), $4.88(\mathrm{~d}, J=16.0 \mathrm{~Hz}, 1 \mathrm{H}), 2.22(\mathrm{~s}, 3 \mathrm{H}) .{ }^{3} \mathbf{C}$ NMR $\left(126 \mathrm{MHz}, \mathrm{DMSO}-d_{6}\right) \delta 174.36,166.42,141.13$, 136.81, 133.75, 132.17, 131.71, 128.94, 128.91, 127.92, 127.79, 127.69, 124.43, 109.22, 53.13, 43.36, 21.01. HRMS (ESI) $m / z$ : Calcd. for $\mathrm{C}_{23} \mathrm{H}_{21} \mathrm{~N}_{2} \mathrm{O}_{2},[\mathrm{M}+\mathrm{H}]^{+}, 357.1598$; found: 357.1586 .

$N$-(1-Benzyl-5-methoxy-2-oxoindolin-3-yl)benzamide (1h)<smiles>COc1ccc2c(c1)C(NC(=O)c1ccccc1)C(=O)N2Cc1ccccc1</smiles>

This compound was prepared according to typical procedure 2.1. Petroleum ether/ethyl acetate $=5: 1$ was used as the eluent for column chromatography. White solid (790 mg, 85\%); m.p. 201.4-202.9 ${ }^{\circ} \mathrm{C}$; ${ }^{1}$ H NMR $\left(500 \mathrm{MHz}, \mathrm{DMSO}-d_{6}\right) \delta 9.48(\mathrm{~d}, J=8.0 \mathrm{~Hz}, 1 \mathrm{H}), 7.93(\mathrm{~d}, J=7.5 \mathrm{~Hz}, 2 \mathrm{H}), 7.61-7.55(\mathrm{~m}, 1 \mathrm{H})$, 7.53-7.48 (m, 2H), $7.44(\mathrm{~d}, J=7.0 \mathrm{~Hz}, 2 \mathrm{H}), 7.34$ (t, $J=7.5 \mathrm{~Hz}, 2 \mathrm{H}), 7.29-7.24(\mathrm{~m}, 1 \mathrm{H}), 6.86(\mathrm{~s}, 1 \mathrm{H})$, $6.77(\mathrm{dd}, J=8.5,2.5 \mathrm{~Hz}, 1 \mathrm{H}), 6.70(\mathrm{~d}, J=8.5 \mathrm{~Hz}, 1 \mathrm{H}), 5.39(\mathrm{~d}, J=8.0 \mathrm{~Hz}, 1 \mathrm{H}), 4.95$ (d, $J=16.0 \mathrm{~Hz}$, $1 \mathrm{H}), 4.87(\mathrm{~d}, J=16.0 \mathrm{~Hz}, 1 \mathrm{H}), 3.66(\mathrm{~s}, 3 \mathrm{H}) .{ }^{13} \mathbf{C}$ NMR $\left(126 \mathrm{MHz}, \mathrm{DMSO}-d_{6}\right) \delta 174.13,166.47,155.92$, $136.87,136.82,133.76,132.17,129.07,128.95,128.90,127.94,127.72,127.68,113.22,110.93,109.89$, 55.98, 53.44, 43.40. HRMS (ESI) $m / z$ : Calcd. for $\mathrm{C}_{23} \mathrm{H}_{21} \mathrm{~N}_{2} \mathrm{O}_{3},[\mathrm{M}+\mathrm{H}]^{+}, 373.1547$; found: 373.1536 .

\section{N-(1-Benzyl-6-fluoro-2-oxoindolin-3-yl)benzamide (1i)}<smiles>O=C(NC1C(=O)N(Cc2ccccc2)c2cc(F)ccc21)c1ccccc1</smiles>

This compound was prepared according to typical procedure 2.1. Petroleum ether/ethyl acetate $=5: 1$ was used as the eluent for column chromatography. White solid (747 mg, 83\%); m.p. 202-203.5 ${ }^{\circ} \mathrm{C} ;{ }^{1} \mathbf{H}$ NMR $\left(500 \mathrm{MHz}, \mathrm{DMSO}-d_{6}\right) \delta 9.51(\mathrm{~d}, J=7.5 \mathrm{~Hz}, 1 \mathrm{H}), 7.92(\mathrm{~d}, J=7.5 \mathrm{~Hz}, 2 \mathrm{H}), 7.58(\mathrm{t}, J=7.5 \mathrm{~Hz}$, 1H), 7.53-7.45 (m, 4H), $7.36(\mathrm{t}, J=7.5 \mathrm{~Hz}, 2 \mathrm{H}), 7.31-7.22(\mathrm{~m}, 2 \mathrm{H}), 6.82-6.73(\mathrm{~m}, 2 \mathrm{H}), 5.36(\mathrm{~d}, J=7.5$ 
$\mathrm{Hz}, 1 \mathrm{H}), 4.99(\mathrm{~d}, J=15.5 \mathrm{~Hz}, 1 \mathrm{H}), 4.91(\mathrm{~d}, J=16.0 \mathrm{~Hz}, 1 \mathrm{H}) .{ }^{13} \mathbf{C}$ NMR $\left(126 \mathrm{MHz}\right.$, DMSO- $\left.d_{6}\right) \delta 174.90$, $166.44,162.93\left(\mathrm{~d}, J_{\mathrm{C}-\mathrm{F}}=241.9 \mathrm{~Hz}\right), 145.29\left(\mathrm{~d}, J_{\mathrm{C}-\mathrm{F}}=12.0 \mathrm{~Hz}\right), 136.45,133.62,132.23,129.00,128.93$, 127.90, 127.83, $124.97\left(\mathrm{~d}, J_{\mathrm{C}-\mathrm{F}}=9.8 \mathrm{~Hz}\right), 123.56\left(\mathrm{~d}, J_{\mathrm{C}-\mathrm{F}}=2.4 \mathrm{~Hz}\right), 108.43\left(\mathrm{~d}, J_{\mathrm{C}-\mathrm{F}}=22.1 \mathrm{~Hz}\right), 98.05(\mathrm{~d}$, $\left.J_{\mathrm{C}-\mathrm{F}}=27.8 \mathrm{~Hz}\right), 52.74,43.44 .{ }^{19} \mathbf{F}$ NMR $\left(471 \mathrm{MHz}, \mathrm{DMSO}-d_{6}\right) \delta-112.60$. HRMS (ESI) $\mathrm{m} / \mathrm{z}$ : Calcd. for $\mathrm{C}_{22} \mathrm{H}_{18} \mathrm{~N}_{2} \mathrm{O}_{2} \mathrm{~F},[\mathrm{M}+\mathrm{H}]^{+}, 361.1347$; found: 361.1344 .

$\mathrm{N}$-(1-Benzyl-6-chloro-2-oxoindolin-3-yl)benzamide (1j)<smiles>O=C(NC1C(=O)N(c2ccccc2)c2cc(Cl)ccc21)c1ccccc1</smiles>

$1 \mathrm{j}$

This compound was prepared according to typical procedure 2.1. Petroleum ether/ethyl acetate $=5: 1$ was used as the eluent for column chromatography. White solid (799 mg, 85\%); m.p. $216.6-218.5^{\circ} \mathrm{C} ;{ }^{1} \mathbf{H}$ NMR $\left(500 \mathrm{MHz}\right.$, DMSO- $\left.d_{6}\right) \delta 9.57$ (d, $\left.J=7.5 \mathrm{~Hz}, 1 \mathrm{H}\right), 7.94-7.89(\mathrm{~m}, 2 \mathrm{H}), 7.61-7.55(\mathrm{~m}, 1 \mathrm{H}), 7.53-7.45$ (m, 4H), $7.36(\mathrm{t}, J=7.5 \mathrm{~Hz}, 2 \mathrm{H}), 7.30-7.23(\mathrm{~m}, 2 \mathrm{H}), 7.03(\mathrm{dd}, J=8.0,1.5 \mathrm{~Hz}, 1 \mathrm{H}), 6.92(\mathrm{~d}, J=1.5 \mathrm{~Hz}$, $1 \mathrm{H}), 5.35(\mathrm{~d}, J=7.5 \mathrm{~Hz}, 1 \mathrm{H}), 5.01(\mathrm{~d}, J=16.0 \mathrm{~Hz}, 1 \mathrm{H}), 4.93(\mathrm{~d}, J=16.0 \mathrm{~Hz}, 1 \mathrm{H}) \cdot{ }^{13} \mathbf{C} \mathbf{N M R}(126 \mathrm{MHz}$, DMSO- $\left.d_{6}\right) \delta 174.61,166.43,145.07,136.41,133.51,133.13,132.27,129.01,128.94,127.91,127.81$, 127.71, 126.77, 125.00, 122.22, 109.61, 52.90, 43.33. HRMS (ESI) m/z: Calcd. for $\mathrm{C}_{22} \mathrm{H}_{18} \mathrm{~N}_{2} \mathrm{O}_{2} \mathrm{Cl}$, $[\mathrm{M}+\mathrm{H}]^{+}, 377.1051$; found: 377.1046 .

\section{$N$-(1-Benzyl-6-bromo-2-oxoindolin-3-yl)benzamide (1k)}<smiles>O=C(NC1C(=O)N(Cc2ccccc2)c2cc(Br)ccc21)c1ccccc1</smiles>

This compound was prepared according to typical procedure 2.1. Petroleum ether/ethyl acetate $=5: 1$ was used as the eluent for column chromatography. White solid ( $892 \mathrm{mg}, 85 \%)$; m.p. $215.6-217.1^{\circ} \mathrm{C}$; ${ }^{1}$ H NMR $\left(400 \mathrm{MHz}\right.$, DMSO- $\left.d_{6}\right) \delta 9.56(\mathrm{~d}, J=7.6 \mathrm{~Hz}, 1 \mathrm{H}), 7.95-7.88(\mathrm{~m}, 2 \mathrm{H}), 7.60-7.55(\mathrm{~m}, 1 \mathrm{H}), 7.54-$ $7.48(\mathrm{~m}, 2 \mathrm{H}), 7.46(\mathrm{~d}, J=7.2 \mathrm{~Hz}, 2 \mathrm{H}), 7.40-7.33(\mathrm{~m}, 2 \mathrm{H}), 7.33-7.27(\mathrm{~m}, 1 \mathrm{H}), 7.21-7.13(\mathrm{~m}, 2 \mathrm{H}), 7.04(\mathrm{~s}$, 1H), $5.33(\mathrm{~d}, J=7.6 \mathrm{~Hz}, 1 \mathrm{H}), 5.01(\mathrm{~d}, J=16.0 \mathrm{~Hz}, 1 \mathrm{H}), 4.93(\mathrm{~d}, J=16.0 \mathrm{~Hz}, 1 \mathrm{H}) .{ }^{13} \mathbf{C} \mathbf{N M R}(101 \mathrm{MHz}$, DMSO- $\left.d_{6}\right) \delta 174.50,166.44,145.21,136.41,133.50,132.28,129.01,128.95,127.90,127.81,127.68$, 127.23, 125.38, 125.17, 121.37, 112.29, 52.96, 43.30. HRMS (ESI) $m / z$ : Calcd. for $\mathrm{C}_{22} \mathrm{H}_{18} \mathrm{~N}_{2} \mathrm{O}_{2} \mathrm{Br}$, $[\mathrm{M}+\mathrm{H}]^{+}, 421.0546$; found: 421.0549 .

\section{$N$-(1-Benzyl-6-methoxy-2-oxoindolin-3-yl)benzamide (1I)}


<smiles>COc1ccc2c(c1)N(Cc1ccccc1)C(=O)C2NC(=O)c1ccccc1</smiles>

This compound was prepared according to typical procedure 2.1. Petroleum ether/ethyl acetate $=5: 1$ was used as the eluent for column chromatography. White solid (772 mg, 83\%); m.p. 201.4-203.2 ${ }^{\circ} \mathrm{C}$; ${ }^{1} \mathbf{H}$ NMR $\left(400 \mathrm{MHz}, \mathrm{CDCl}_{3}\right) \delta 7.83(\mathrm{~d}, J=7.2 \mathrm{~Hz}, 2 \mathrm{H}), 7.53-7.47(\mathrm{~m}, 1 \mathrm{H}), 7.41(\mathrm{t}, J=7.6 \mathrm{~Hz}, 2 \mathrm{H}), 7.38$ $7.27(\mathrm{~m}, 6 \mathrm{H}), 7.02(\mathrm{~d}, J=7.2 \mathrm{~Hz}, 1 \mathrm{H}), 6.51(\mathrm{dd}, J=8.4,2.4 \mathrm{~Hz}, 1 \mathrm{H}), 6.33(\mathrm{~d}, J=2.0 \mathrm{~Hz}, 1 \mathrm{H}), 5.51(\mathrm{~d}$, $J=7.2 \mathrm{~Hz}, 1 \mathrm{H}), 4.98(\mathrm{~d}, J=15.6 \mathrm{~Hz}, 1 \mathrm{H}), 4.85(\mathrm{~d}, J=15.6 \mathrm{~Hz}, 1 \mathrm{H}), 3.72(\mathrm{~s}, 3 \mathrm{H}) .{ }^{13} \mathbf{C}$ NMR $(101 \mathrm{MHz}$, $\left.\mathrm{CDCl}_{3}\right) \delta 175.02,167.65,160.80,144.32,135.41,133.25,131.93,128.87,128.56,127.77,127.40$, 127.29, 125.51, 118.44, 106.56, 97.71, 55.47, 52.77, 44.25. HRMS (ESI) $m / z$ : Calcd. for $\mathrm{C}_{23} \mathrm{H}_{21} \mathrm{~N}_{2} \mathrm{O}_{3}$, $[\mathrm{M}+\mathrm{H}]^{+}, 373.1547$; found: 373.1536 .

\section{$N$-(1-Benzyl-7-fluoro-2-oxoindolin-3-yl)benzamide (1m)}<smiles>O=C(NC1C(=O)N(Cc2ccccc2)c2c(F)cccc21)c1ccccc1</smiles>

This compound was prepared according to typical procedure 2.1. Petroleum ether/ethyl acetate $=5: 1$ was used as the eluent for column chromatography. White solid (765 mg, 85\%); m.p.184.6-186.3 ${ }^{\circ} \mathrm{C} ;{ }^{1} \mathbf{H}$ NMR $\left(500 \mathrm{MHz}\right.$, DMSO- $\left.d_{6}\right) \delta 9.61(\mathrm{~d}, J=7.5 \mathrm{~Hz}, 1 \mathrm{H}), 7.96-7.91(\mathrm{~m}, 2 \mathrm{H}), 7.60-7.56(\mathrm{~m}, 1 \mathrm{H}), 7.53-7.49$ (m, 2H), $7.42(\mathrm{~d}, J=7.5 \mathrm{~Hz}, 2 \mathrm{H}), 7.37-7.24(\mathrm{~m}, 4 \mathrm{H}), 7.12-7.06(\mathrm{~m}, 2 \mathrm{H}), 7.04-6.97(\mathrm{~m}, 1 \mathrm{H}), 5.43(\mathrm{~d}, J=$ $7.5 \mathrm{~Hz}, 1 \mathrm{H}), 5.08(\mathrm{~d}, J=16.0 \mathrm{~Hz}, 1 \mathrm{H}), 4.98(\mathrm{~d}, J=16.5 \mathrm{~Hz}, 1 \mathrm{H}) \cdot{ }^{13} \mathbf{C}$ NMR $\left(126 \mathrm{MHz}\right.$, DMSO- $\left.d_{6}\right) \delta$ 174.35, 166.46, $147.06\left(\mathrm{~d}, J_{\mathrm{C}-\mathrm{F}}=242.3 \mathrm{~Hz}\right), 137.71,133.50,132.30,131.06\left(\mathrm{~d}, J_{\mathrm{C}-\mathrm{F}}=3.0 \mathrm{~Hz}\right), 129.86$ $\left(\mathrm{d}, J_{\mathrm{C}-\mathrm{F}}=8.8 \mathrm{~Hz}\right), 128.95,128.88,127.92,127.58,127.07,123.68\left(\mathrm{~d}, J_{\mathrm{C}-\mathrm{F}}=6.3 \mathrm{~Hz}\right), 119.92\left(\mathrm{~d}, J_{\mathrm{C}-\mathrm{F}}=\right.$ $2.1 \mathrm{~Hz}), 116.81\left(\mathrm{~d}, J_{\mathrm{C}-\mathrm{F}}=19.2 \mathrm{~Hz}\right), 53.43,45.19,45.16 .{ }^{19} \mathbf{F}$ NMR $\left(471 \mathrm{MHz}, \mathrm{DMSO}-d_{6}\right) \delta-135.74$. HRMS (ESI) $m / z$ : Calcd. for $\mathrm{C}_{22} \mathrm{H}_{18} \mathrm{~N}_{2} \mathrm{O}_{2} \mathrm{~F}$, [M+H]+, 361.1347; found: 361.1341 .

$\mathrm{N}$-(1-Benzyl-7-chloro-2-oxoindolin-3-yl)benzamide (1n)<smiles>O=C(NC1C(=O)N(Cc2ccccc2)c2c(Cl)cccc21)c1ccccc1</smiles>

This compound was prepared according to typical procedure 2.1. Petroleum ether/ethyl acetate $=5: 1$ was used as the eluent for column chromatography. White solid (752 mg, 80\%); m.p. $222.8-223.4{ }^{\circ} \mathrm{C}$; 
${ }^{1}$ H NMR $\left(500 \mathrm{MHz}\right.$, DMSO- $\left.d_{6}\right) \delta 9.63(\mathrm{~d}, J=7.5 \mathrm{~Hz}, 1 \mathrm{H}), 7.96-7.89(\mathrm{~m}, 2 \mathrm{H}), 7.61-7.56(\mathrm{~m}, 1 \mathrm{H}), 7.54-$ $7.48(\mathrm{~m}, 2 \mathrm{H}), 7.39(\mathrm{~d}, J=7.5 \mathrm{~Hz}, 2 \mathrm{H}), 7.36-7.30(\mathrm{~m}, 2 \mathrm{H}), 7.28-7.19(\mathrm{~m}, 3 \mathrm{H}), 7.02(\mathrm{dd}, J=8.0,7.5 \mathrm{~Hz}$, 1H), $5.42(\mathrm{~d}, J=7.5 \mathrm{~Hz}, 1 \mathrm{H}), 5.31(\mathrm{~d}, J=17.0 \mathrm{~Hz}, 1 \mathrm{H}), 5.24(\mathrm{~d}, J=16.5 \mathrm{~Hz}, 1 \mathrm{H}) \cdot{ }^{13} \mathbf{C}$ NMR $(126 \mathrm{MHz}$, DMSO- $\left.d_{6}\right) \delta 175.18,166.41,139.34,138.40,133.44,132.33,131.23,130.89,128.97,128.83,127.92$, 127.25, 126.58, 124.09, 122.58, 114.36, 53.11, 44.87. HRMS (ESI) m/z: Calcd. for $\mathrm{C}_{22} \mathrm{H}_{18} \mathrm{~N}_{2} \mathrm{O}_{2} \mathrm{Cl}$, $[\mathrm{M}+\mathrm{H}]^{+}, 377.1051$; found: 377.1049 .

$N$-(1-Benzyl-7-bromo-2-oxoindolin-3-yl)benzamide (10)<smiles>O=C(NC1C(=O)N(Cc2ccccc2)c2c(Br)cccc21)c1ccccc1</smiles>

This compound was prepared according to typical procedure 2.1. Petroleum ether/ethyl acetate $=5: 1$ was used as the eluent for column chromatography. White solid (840 mg, 80\%); m.p. 209.6-211.8 ${ }^{\circ} \mathrm{C}$; ${ }^{1}$ H NMR (400 MHz, DMSO- $\left.d_{6}\right) \delta 9.63(\mathrm{~d}, J=7.2 \mathrm{~Hz}, 1 \mathrm{H}), 7.95-7.90(\mathrm{~m}, 2 \mathrm{H}), 7.60-.57(\mathrm{~m}, 1 \mathrm{H}), 7.54-$ $7.49(\mathrm{~m}, 2 \mathrm{H}), 7.41-7.37(\mathrm{~m}, 3 \mathrm{H}), 7.36-7.32(\mathrm{~m}, 2 \mathrm{H}), 7.29-7.24(\mathrm{~m}, 2 \mathrm{H}), 7.00-6.93(\mathrm{~m}, 1 \mathrm{H}), 5.43(\mathrm{~d}, J=$ $7.2 \mathrm{~Hz}, 1 \mathrm{H}), 5.35(\mathrm{~d}, J=17.2 \mathrm{~Hz}, 1 \mathrm{H}), 5.29$ (d, $J=16.8 \mathrm{~Hz}, 1 \mathrm{H}) \cdot{ }^{13} \mathbf{C}$ NMR $\left(101 \mathrm{MHz}\right.$, DMSO- $\left.d_{6}\right) \delta$ 175.37, 166.41, 140.76, 138.32, 134.25, 133.44, 132.33, 131.57, 128.97, 128.80, 127.91, 127.19, 126.59, 124.49, 123.04, 101.80, 53.06, 44.60. HRMS (ESI) $m / z$ : Calcd. for $\mathrm{C}_{22} \mathrm{H}_{18} \mathrm{~N}_{2} \mathrm{O}_{2} \mathrm{Br}$, [M+H]+, 421.0546; found: 421.0550 .

\section{$N$-(1-Benzyl-2-oxo-7-(trifluoromethyl)indolin-3-yl)benzamide (1p)}<smiles>O=C(NC1C(=O)N(Cc2ccccc2)c2c1cccc2C(F)(F)F)c1ccccc1</smiles>

This compound was prepared according to typical procedure 2.1. Petroleum ether/ethyl acetate $=5: 1$ was used as the eluent for column chromatography. White solid (768 mg, 75\%); m.p. $183.5-186.4{ }^{\circ} \mathrm{C}$; ${ }^{1} \mathbf{H}$ NMR $\left(400 \mathrm{MHz}, \mathrm{CDCl}_{3}\right) \delta 7.80(\mathrm{~d}, J=7.6 \mathrm{~Hz}, 1 \mathrm{H}), 7.70(\mathrm{~d}, J=7.6 \mathrm{~Hz}, 2 \mathrm{H}), 7.56(\mathrm{~d}, J=8.4 \mathrm{~Hz}$, $1 \mathrm{H}), 7.51(\mathrm{~d}, J=7.6 \mathrm{~Hz}, 1 \mathrm{H}), 7.42(\mathrm{t}, J=7.6 \mathrm{~Hz}, 1 \mathrm{H}), 7.31-7.21(\mathrm{~m}, 6 \mathrm{H}), 7.20-7.15(\mathrm{~m}, 1 \mathrm{H}), 7.11(\mathrm{t}, J$ $=7.6 \mathrm{~Hz}, 1 \mathrm{H}), 5.22(\mathrm{~s}, 2 \mathrm{H}), 5.20(\mathrm{~s}, 1 \mathrm{H}) .{ }^{13} \mathbf{C} \mathbf{N M R}\left(101 \mathrm{MHz}, \mathrm{CDCl}_{3}\right) \delta 176.42,167.37,140.82,136.06$, 132.31, 132.02, 129.47, 128.50, 128.44, 127.85, 127.29, 127.14 (d, $\left.J_{\mathrm{C}-\mathrm{F}}=6.2 \mathrm{~Hz}\right), 126.96,125.80,122.76$, $113.15\left(\mathrm{~d}, J_{\mathrm{C}-\mathrm{F}}=32.9 \mathrm{~Hz}\right), 52.14,46.17 .{ }^{19} \mathbf{F}$ NMR $\left(376 \mathrm{MHz}, \mathrm{CDCl}_{3}\right) \delta-54.72$. HRMS (ESI) $\mathrm{m} / \mathrm{z}$ : Calcd. for $\mathrm{C}_{23} \mathrm{H}_{18} \mathrm{~N}_{2} \mathrm{O}_{2} \mathrm{~F}_{3},[\mathrm{M}+\mathrm{H}]^{+}, 411.1315$; found: 411.1313 .

$N$-(1-Benzyl-5,7-dimethyl-2-oxoindolin-3-yl)benzamide (1q) 
<smiles>Cc1cc(C)c2c(c1)C(NC(=O)c1ccccc1)C(=O)N2Cc1ccccc1</smiles>

This compound was prepared according to typical procedure 2.1. Petroleum ether/ethyl acetate $=5: 1$ was used as the eluent for column chromatography. White solid $(749 \mathrm{mg}, 81 \%)$; m.p. $230.1-232.5^{\circ} \mathrm{C}$; ${ }^{1} \mathbf{H}$ NMR $\left(400 \mathrm{MHz}, \mathrm{CDCl}_{3}\right) \delta 7.82(\mathrm{~d}, J=7.6 \mathrm{~Hz}, 2 \mathrm{H}), 7.47(\mathrm{t}, J=7.2 \mathrm{~Hz}, 1 \mathrm{H}), 7.41-7.30$ (m, 5H), 7.26$7.22(\mathrm{~m}, 2 \mathrm{H}), 7.08(\mathrm{~s}, 1 \mathrm{H}), 6.78(\mathrm{~s}, 1 \mathrm{H}), 5.46(\mathrm{~d}, J=7.2 \mathrm{~Hz}, 1 \mathrm{H}), 5.27-5.12(\mathrm{~m}, 2 \mathrm{H}), 2.22(\mathrm{~d}, J=3.2 \mathrm{~Hz}$, 6H). ${ }^{13}$ C NMR (101 MHz, $\left.\mathrm{CDCl}_{3}\right) \delta 175.61,167.53,138.43,137.39,133.51,133.12,132.85,131.87$, $128.92,128.51,127.43,127.37,127.24,125.76,123.15,119.82,53.08,45.44,20.69,18.54$. HRMS (ESI) $m / z$ : Calcd. for $\mathrm{C}_{24} \mathrm{H}_{23} \mathrm{~N}_{2} \mathrm{O}_{2},[\mathrm{M}+\mathrm{H}]^{+}, 371.1754$; found: 371.1745 .

\section{$N$-(1-Benzyl-2-oxoindolin-3-yl)-2-naphthamide (1r)}<smiles>O=C(NC1C(=O)N(Cc2ccccc2)c2ccccc21)c1ccc2ccccc2c1</smiles>

This compound was prepared according to typical procedure 2.2. Petroleum ether/ethyl acetate $=4: 1$ was used as the eluent for column chromatography. White solid (755 mg, 76\%); m.p. 190.4-191.7 ${ }^{\circ} \mathrm{C}$; ${ }^{1}$ H NMR (400 MHz, DMSO- $\left.d_{6}\right) \delta 9.67$ (d, $J=7.6$ Hz, 1H), 8.55 (s, 1H), 8.06-7.97 (m, 4H), 7.67-7.59 (m, 2H), $7.48(\mathrm{~d}, J=7.2 \mathrm{~Hz}, 2 \mathrm{H}), 7.39-7.33(\mathrm{~m}, 2 \mathrm{H}), 7.31-7.25(\mathrm{~m}, 2 \mathrm{H}), 7.24-7.18(\mathrm{~m}, 1 \mathrm{H}), 7.05-6.97(\mathrm{~m}$ , 1H), $6.82(\mathrm{~d}, J=7.6 \mathrm{~Hz}, 1 \mathrm{H}), 5.50(\mathrm{~d}, J=7.6 \mathrm{~Hz}, 1 \mathrm{H}), 5.01(\mathrm{~d}, J=16.0 \mathrm{~Hz}, 1 \mathrm{H}), 4.92(\mathrm{~d}, J=16.0 \mathrm{~Hz}$, $1 \mathrm{H}) .{ }^{13} \mathbf{C}$ NMR $\left(101 \mathrm{MHz}, \mathrm{DMSO}-d_{6}\right) \delta 174.51,166.59,143.57,136.75,134.79,132.59,131.14,129.41$, 128.99, 128.86, 128.54, 128.41, 128.34, 128.15, 127.79, 127.74, 127.36, 124.59, 123.83, 122.70, 109.48, 53.18, 43.38. HRMS (ESI) $m / z$ : Calcd. for $\mathrm{C}_{26} \mathrm{H}_{21} \mathrm{~N}_{2} \mathrm{O}_{2}$, [M+H] $]^{+}, 393.1598$; found:393.1599.

\section{$N$-(1-Benzyl-2-oxoindolin-3-yl)-1H-indole-3-carboxamide (1s)}<smiles>O=C(NC1C(=O)N(Cc2ccccc2)c2ccccc21)c1c[nH]c2ccccc12</smiles> 
This compound was prepared according to typical procedure 2.2. Petroleum ether/ethyl acetate $=4: 1$ was used as the eluent for column chromatography. White solid (695 mg, 73\%); m.p. 185.5-186.4 ${ }^{\circ} \mathrm{C}$; ${ }^{1} \mathbf{H}$ NMR (500 MHz, $\left.\mathrm{CDCl}_{3}\right) \delta 9.27(\mathrm{~s}, 1 \mathrm{H}), 8.07(\mathrm{~d}, J=7.5 \mathrm{~Hz}, 1 \mathrm{H}), 7.46(\mathrm{~s}, 1 \mathrm{H}), 7.35(\mathrm{~d}, J=7.5 \mathrm{~Hz}$, 2H), 7.31-7.27 (m, 3H), 7.25-7.20 (m, 1H), 7.17 (d, $J=8.0 \mathrm{~Hz}, 2 \mathrm{H}), 7.15-7.05(\mathrm{~m}, 3 \mathrm{H}), 6.98(\mathrm{t}, J=7.5$ $\mathrm{Hz}, 1 \mathrm{H}), 6.84(\mathrm{~d}, J=7.5 \mathrm{~Hz}, 1 \mathrm{H}), 6.71(\mathrm{~d}, J=7.5 \mathrm{~Hz}, 1 \mathrm{H}), 5.35(\mathrm{~d}, J=7.5 \mathrm{~Hz}, 1 \mathrm{H}), 4.95(\mathrm{~d}, J=15.5 \mathrm{~Hz}$, $1 \mathrm{H}), 4.79(\mathrm{~d}, J=16.0 \mathrm{~Hz}, 1 \mathrm{H}) .{ }^{13} \mathbf{C} \mathbf{N M R}\left(126 \mathrm{MHz}, \mathrm{CDCl}_{3}\right) \delta 175.80,165.57,142.66,136.15,135.48$, $128.86,128.82$, 128.68, 127.69, 127.28, 126.92, 125.28, 124.17, 123.18, 122.65, 121.50, 120.55, 112.06, 109.78, 109.40, 53.16, 44.11. HRMS (ESI) $m / z$ : Calcd. for $\mathrm{C}_{24} \mathrm{H}_{20} \mathrm{~N}_{3} \mathrm{O}_{2},[\mathrm{M}+\mathrm{H}]^{+}, 382.1550$; found: 382.1536.

\section{$N$-(1-Benzyl-2-oxoindolin-3-yl)thiophene-2-carboxamide (1t)}<smiles>O=C(NC1C(=O)N(Cc2ccccc2)c2ccccc21)c1cccs1</smiles>

This compound was prepared according to typical procedure 2.2. Petroleum ether/ethyl acetate $=5: 1$ was used as the eluent for column chromatography. White solid (609 mg, 70\%); m.p. 189.5-190.1 ${ }^{\circ} \mathrm{C}$; ${ }^{1}$ H NMR (400 MHz, DMSO- $\left.d_{6}\right) \delta 9.49(\mathrm{~d}, J=8.0 \mathrm{~Hz}, 1 \mathrm{H}), 7.86(\mathrm{dd}, J=4.0,1.2 \mathrm{~Hz}, 1 \mathrm{H}), 7.82(\mathrm{dd}, J=$ 5.2, 1.2 Hz, 1H), 7.48-7.41 (m, 2H), 7.38-7.31 (m, 2H), 7.30-7.17 (m, 4H), 7.04-6.97 (m, 1H), $6.82(\mathrm{~d}, J$ $=7.6 \mathrm{~Hz}, 1 \mathrm{H}), 5.42(\mathrm{~d}, J=7.6 \mathrm{~Hz}, 1 \mathrm{H}), 4.96(\mathrm{~d}, J=16.0 \mathrm{~Hz}, 1 \mathrm{H}), 4.90(\mathrm{~d}, J=16.0 \mathrm{~Hz}, 1 \mathrm{H}) .{ }^{13} \mathbf{C} \mathbf{N M R}$ $\left(101 \mathrm{MHz}, \mathrm{DMSO}-d_{6}\right) \delta 174.36,161.53,143.49,139.22,136.71,132.01,129.41,128.99,128.93,128.57$, 127.74, 127.57, 123.86, 122.73, 109.51, 52.91, 43.37. HRMS (ESI) $m / z$ : Calcd. for $\mathrm{C}_{20} \mathrm{H}_{17} \mathrm{~N}_{2} \mathrm{O}_{2} \mathrm{~S}$, $[\mathrm{M}+\mathrm{H}]^{+}, 349.1005$; found: 349.0990 .

\section{$N$-(1-Benzyl-2-oxoindolin-3-yl)furan-2-carboxamide (1u)}<smiles>O=C(NC1C(=O)N(Cc2ccccc2)c2ccccc21)c1ccco1</smiles>

This compound was prepared according to typical procedure 2.2. Petroleum ether/ethyl acetate $=5: 1$ was used as the eluent for column chromatography. White solid (581 mg, 70\%); m.p. 187.6-189.4 ${ }^{\circ} \mathrm{C}$; ${ }^{1}$ H NMR (400 MHz, DMSO- $\left.d_{6}\right) \delta 9.38(\mathrm{~d}, J=8.0 \mathrm{~Hz}, 1 \mathrm{H}), 7.90(\mathrm{dd}, J=1.6,0.8 \mathrm{~Hz}, 1 \mathrm{H}), 7.46-7.41(\mathrm{~m}$, 2H), 7.36-7.30 (m, 2H), 7.29-7.16 (m, 4H), 7.01-6.96 (m, 1H), $6.78(\mathrm{~d}, J=7.6 \mathrm{~Hz}, 1 \mathrm{H}), 6.66(\mathrm{dd}, J=$ 3.6, 1.6 Hz, 1H), 5.39 (d, $J=8.0 \mathrm{~Hz}, 1 \mathrm{H}), 4.97(\mathrm{~d}, J=16.0 \mathrm{~Hz}, 1 \mathrm{H}), 4.87(\mathrm{~d}, J=16.0 \mathrm{~Hz}, 1 \mathrm{H}) .{ }^{13} \mathbf{C}$ NMR $\left(101 \mathrm{MHz}, \mathrm{DMSO}-d_{6}\right) \delta 174.30,158.07,147.61,146.00,143.49,136.67,128.95,128.88,127.70,127.56$, 123.81, 122.67, 114.75, 112.49, 109.49, 52.45, 43.36. HRMS (ESI) $m / z$ : Calcd. for $\mathrm{C}_{20} \mathrm{H}_{17} \mathrm{~N}_{2} \mathrm{O}_{3},[\mathrm{M}+\mathrm{H}]^{+}$, 331.1088; found: 331.1087 . 
<smiles>O=C(NC1C(=O)N(Cc2ccccc2)c2ccccc21)C1CC1</smiles>

This compound was prepared according to typical procedure 2.2. Petroleum ether/ethyl acetate $=4: 1$ was used as the eluent for column chromatography. White solid (482 mg, 63\%); m.p. 190.4-191.7 ${ }^{\circ} \mathrm{C}$; ${ }^{1}$ H NMR $\left(400 \mathrm{MHz}, \mathrm{CDCl}_{3}\right) \delta$ 7.38-7.29 (m, 5H), 7.21-715 (m, 1H), 7.05-6.99 (m, 1H), 6.71 (d, $J=8.0$ $\mathrm{Hz}, 1 \mathrm{H}), 6.49$ (d, $J=7.2 \mathrm{~Hz}, 1 \mathrm{H}), 5.43$ (d, $J=7.6 \mathrm{~Hz}, 1 \mathrm{H}), 4.98$ (d, $J=16.0 \mathrm{~Hz}, 1 \mathrm{H}), 4.84$ (d, $J=15.6$ $\mathrm{Hz}, 1 \mathrm{H}), 1.54-1.46(\mathrm{~m}, 1 \mathrm{H}), 1.09-1.00(\mathrm{~m}, 2 \mathrm{H}), 0.86-0.77(\mathrm{~m}, 2 \mathrm{H}) .{ }^{13} \mathbf{C} \mathbf{N M R}\left(101 \mathrm{MHz}, \mathrm{CDCl}_{3}\right) \delta 174.56$, 174.20, 142.83, 135.46, 129.08, 128.83, 127.72, 127.34, 126.74, 124.68, 123.05, 109.32, 52.89, 44.14, 14.62, 7.97, 7.90. HRMS (ESI) $m / z$ : Calcd. for $\mathrm{C}_{19} \mathrm{H}_{17} \mathrm{~N}_{2} \mathrm{O}_{2},[\mathrm{M}-\mathrm{H}]^{-}, 305.1296$; found: 305.1297 .

\section{$N$-(1-Benzyl-2-oxoindolin-3-yl)cyclopentanecarboxamide (1w)}<smiles>O=C(NC1C(=O)N(Cc2ccccc2)c2ccccc21)C1CCCC1</smiles>

This compound was prepared according to typical procedure 2.2. Petroleum ether/ethyl acetate $=4: 1$ was used as the eluent for column chromatography. White solid (492 mg, 59\%); m.p. 187.5-189.7 ${ }^{\circ} \mathrm{C}$; ${ }^{1} \mathbf{H}$ NMR $\left(400 \mathrm{MHz}, \mathrm{DMSO}-d_{6}\right) \delta 8.71(\mathrm{~d}, J=8.0 \mathrm{~Hz}, 1 \mathrm{H}), 7.39(\mathrm{~d}, J=7.2 \mathrm{~Hz}, 2 \mathrm{H}), 7.34-7.29(\mathrm{~m}, 2 \mathrm{H})$, 7.28-7.23 (m, 1H), 7.20-7.13 (m, 2H), 7.01-6.95 (m, 1H), $6.77(\mathrm{~d}, J=7.6 \mathrm{~Hz}, 1 \mathrm{H}), 5.23(\mathrm{~d}, J=8.0 \mathrm{~Hz}$, $1 \mathrm{H}), 4.92(\mathrm{~d}, J=16.0 \mathrm{~Hz}, 1 \mathrm{H}), 4.86$ (d, $J=16.0 \mathrm{~Hz}, 1 \mathrm{H}), 2.71-2.61(\mathrm{~m}, 1 \mathrm{H}), 1.84-1.74(\mathrm{~m}, 2 \mathrm{H}), 1.72-$ $1.59(\mathrm{~m}, 4 \mathrm{H}), 1.55-1.46(\mathrm{~m}, 2 \mathrm{H}) .{ }^{13} \mathrm{C}$ NMR (101 MHz, DMSO- $\left.d_{6}\right) \delta 175.97,174.59,143.34,136.74$, $128.96,128.73,128.04,127.69,123.60,122.66,109.40,52.45,44.23,43.25,30.39,26.18,26.15$. HRMS (ESI) $m / z$ : Calcd. for $\mathrm{C}_{21} \mathrm{H}_{23} \mathrm{~N}_{2} \mathrm{O}_{2}$, [M+H] $]^{+}, 335.1754$; found: 335.1741 .

\section{$N$-(1-Benzyl-2-oxoindolin-3-yl)tetrahydro-2H-pyran-4-carboxamide (1x)}<smiles>[X]c1ccccc1N1C(=O)C(NC(=O)C2CCOCC2)c2ccccc21</smiles>

This compound was prepared according to typical procedure 2.2. Petroleum ether/ethyl acetate $=4: 1$ was used as the eluent for column chromatography. White solid (116 mg, 33\%); m.p. 174.6-176.9 ${ }^{\circ} \mathrm{C}$; 
${ }^{1} \mathbf{H}$ NMR $\left(500 \mathrm{MHz}, \mathrm{CDCl}_{3}\right) \delta$ 7.36-7.27 (m, 6H), 7.23-7.16 (m, 1H), 7.06-6.99 (m, 1H), $6.73(\mathrm{~d}, J=7.5$ $\mathrm{Hz}, 1 \mathrm{H}), 6.29$ (d, $J=5.0 \mathrm{~Hz}, 1 \mathrm{H}), 5.41$ (d, $J=6.5 \mathrm{~Hz}, 1 \mathrm{H}), 4.97$ (d, $J=16.0 \mathrm{~Hz}, 1 \mathrm{H}), 4.84$ (d, $J=15.5$ $\mathrm{Hz}, 1 \mathrm{H}), 4.07-3.96(\mathrm{~m}, 2 \mathrm{H}), 3.46-3.36(\mathrm{~m}, 2 \mathrm{H}), 2.54-2.43(\mathrm{~m}, 1 \mathrm{H}), 1.92-1.79(\mathrm{~m}, 4 \mathrm{H}) .{ }^{13} \mathbf{C}$ NMR $(126$ $\left.\mathrm{MHz}, \mathrm{CDCl}_{3}\right) \delta 174.80,174.35,142.88,135.38,129.18,128.85,127.77,127.34,126.53,124.43,123.12$, 109.40, 67.16, 67.14, 52.60, 44.15, 41.77, 29.24, 29.10. HRMS (ESI) m/z: Calcd. for $\mathrm{C}_{21} \mathrm{H}_{23} \mathrm{~N}_{2} \mathrm{O}_{3}$, $[\mathrm{M}+\mathrm{H}]^{+}, 351.1703$; found: 351.1710 .

\section{Ethyl (1-benzyl-2-oxoindolin-3-yl)carbamate (1y)}<smiles>CCOC(=O)NC1C(=O)N(Cc2ccccc2)c2ccccc21</smiles>

This compound was prepared according to typical procedure 2.1. Petroleum ether/ethyl acetate $=5: 1$ was used as the eluent for column chromatography. White solid (1.02 g, 83\%); m.p.152.5-153.9 ${ }^{\circ} \mathrm{C} .{ }^{1} \mathbf{H}$ NMR (400 MHz, $\left.\mathrm{CDCl}_{3}\right) \delta$ 7.39-7.29 (m, 5H), 7.28-7.22 (m, 1H), $7.18(\mathrm{t}, J=7.6 \mathrm{~Hz}, 1 \mathrm{H}), 7.05-7.00(\mathrm{~m}$, $1 \mathrm{H}), 6.71(\mathrm{~d}, J=8.0 \mathrm{~Hz}, 1 \mathrm{H}), 5.47(\mathrm{~d}, J=6.8 \mathrm{~Hz}, 1 \mathrm{H}), 5.16(\mathrm{~d}, J=4.8 \mathrm{~Hz}, 1 \mathrm{H}), 4.96(\mathrm{~d}, J=16.0 \mathrm{~Hz}$, $1 \mathrm{H}), 4.86(\mathrm{~d}, J=15.6 \mathrm{~Hz}, 1 \mathrm{H}), 4.17(\mathrm{~d}, J=6.8 \mathrm{~Hz}, 2 \mathrm{H}), 1.25(\mathrm{~s}, 3 \mathrm{H}) \cdot{ }^{13} \mathbf{C} \mathbf{N M R}\left(126 \mathrm{MHz}, \mathrm{CDCl}_{3}\right) \delta$ 174.46, 156.47, 142.84, 135.46, 129.20, 128.80, 127.70, 127.34, 126.43, 124.41, 122.98, 109.35, 61.65, 53.87, 44.10, 14.49. HRMS (ESI) $m / z$ : Calcd. for $\mathrm{C}_{18} \mathrm{H}_{19} \mathrm{~N}_{2} \mathrm{O}_{3}$, [M+H $]^{+}, 311.1390$; found: 311.1395 .

\section{Typical procedures and characterization data for compounds 3a-3y}

\subsection{General procedure for the synthesis of compounds 3a-3y}

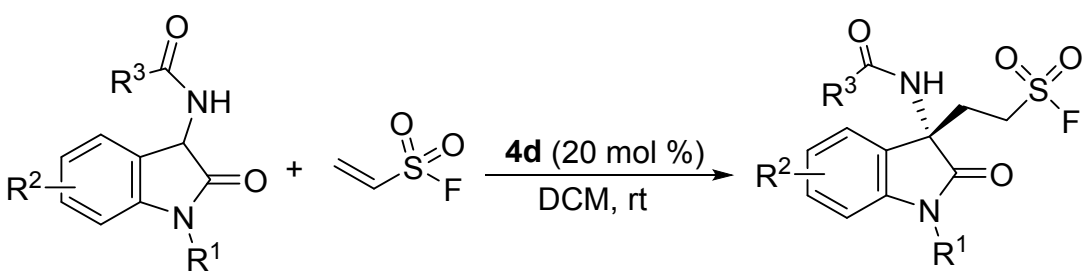

A solution of 3-benzamido-2-oxindole 1a-1y (0.1 mmol), ethylene sulfonyl fluoride $\mathbf{2 a}(13.2 \mathrm{mg}, 0.12$ mmol) and catalyst $4 \mathbf{d}(12.6 \mathrm{mg}, 0.02 \mathrm{mmol})$ in $\mathrm{CH}_{2} \mathrm{Cl}_{2}(1.0 \mathrm{~mL})$ was stirred at $\mathrm{rt}$ for $12 \mathrm{~h}$. After the solvent was evaporated under vacuum, the residue was purified by flash column chromatography over silica gel (Petroleum ether/ethyl acetate $=8: 1$ ) to afford the product $\mathbf{3 a}-\mathbf{3 y}$.

3.2 General procedure for the synthesis of racemic products $3 a-3 y$<smiles>[R][R]1ccc2c(c1)C(NC([R])=O)C(=[OH+])N2[R]</smiles>

A solution of 3-benzamido-2-oxindole 1a-1y (0.1 mmol), ethylene sulfonyl fluoride $2 \mathbf{a}(13.2 \mathrm{mg}, 0.12$ $\mathrm{mmol}), \mathrm{NaHCO}_{3}(16.8 \mathrm{mg}, 0.2 \mathrm{mmol})$ and $\mathrm{Et}_{3} \mathrm{~N}(3 \mathrm{mg}, 0.02 \mathrm{mmol})$ in $\mathrm{CH}_{2} \mathrm{Cl}_{2}(1.0 \mathrm{~mL})$ was stirred at $\mathrm{rt}$ for $12 \mathrm{~h}$. After the solvent was evaporated under vacuum, the residue was purified by flash column 
chromatography over silica gel (Petroleum ether/ethyl acetate $=8: 1$ ) to afford the racemic products 3a$3 y$.

3.3 Characterizations of products $3 a-3 y$

(S)-2-(3-Benzamido-1-benzyl-2-oxoindolin-3-yl)ethane-1-sulfonyl fluoride (3a)<smiles>O=C(N[C@]1(CCS(=O)(=O)F)C(=O)N(Cc2ccccc2)c2ccccc21)c1ccccc1</smiles>

$3 a$

Petroleum ether/ethyl acetate $=8: 1$ was used as the eluent for column chromatography. White solid (44.5 mg, 98\%), m.p. 172.1-173.5 ${ }^{\circ} \mathrm{C} .{ }^{1} \mathbf{H}$ NMR (500 MHz, $\left.\mathrm{CDCl}_{3}\right) \delta$ 7.79-7.72 (m, 2H), 7.51-7.46 (m, $1 \mathrm{H}), 7.43-7.34(\mathrm{~m}, 7 \mathrm{H}), 7.33-7.26(\mathrm{~m}, 2 \mathrm{H}), 7.25-7.20(\mathrm{~m}, 1 \mathrm{H}), 7.05(\mathrm{td}, J=7.5,0.5 \mathrm{~Hz}, 1 \mathrm{H}), 6.79(\mathrm{~d}, J$ $=8.0 \mathrm{~Hz}, 1 \mathrm{H}), 5.02(\mathrm{~d}, J=16.0 \mathrm{~Hz}, 1 \mathrm{H}), 4.96(\mathrm{~d}, J=16.0 \mathrm{~Hz}, 1 \mathrm{H}), 3.72-3.63(\mathrm{~m}, 1 \mathrm{H}), 3.49-3.40(\mathrm{~m}$, 1H), $2.82(\mathrm{ddd}, J=14.0,10.0,5.0 \mathrm{~Hz}, 1 \mathrm{H}), 2.49(\mathrm{ddd}, J=14.5,10.0,5.0 \mathrm{~Hz}, 1 \mathrm{H}) .{ }^{13} \mathrm{C} \mathrm{NMR}(126 \mathrm{MHz}$, $\left.\mathrm{CDCl}_{3}\right) \delta 175.02,166.71,142.19,135.28,132.35,132.26,129.81,128.99,128.63,128.31,127.89$, 127.34, 127.29, 123.60, 123.01, 109.99, 59.92, $44.95\left(\mathrm{~d}, J_{\mathrm{C}-\mathrm{F}}=18.1 \mathrm{~Hz}\right), 44.39,30.40 .{ }^{19} \mathbf{F}$ NMR $(471$ $\left.\mathrm{MHz}, \mathrm{CDCl}_{3}\right) \delta$ 53.31. HRMS (ESI) $m / z$ : Calcd. for $\mathrm{C}_{24} \mathrm{H}_{21} \mathrm{FN}_{2} \mathrm{O}_{4} \mathrm{SNa},[\mathrm{M}+\mathrm{Na}]^{+}, 475.3274$; found: 475.3276. $[\alpha]_{\mathrm{D}}{ }^{20}=+25.8\left(\mathrm{c} 1.0, \mathrm{CH}_{2} \mathrm{Cl}_{2}\right)$. Chiral HPLC analysis (Chiral pak AS-H, $i$-PrOH/n-hexane $=$ $20: 80$, flow rate $=0.5 \mathrm{~mL} / \mathrm{min}$, wave length $=254 \mathrm{~nm}), \mathrm{t}_{\mathrm{R}}($ minor enantiomer $)=16.959 \mathrm{~min}, \mathrm{t}_{\mathrm{R}}($ major enantiomer) $=32.849 \mathrm{~min}, 99 \%$ ee. 


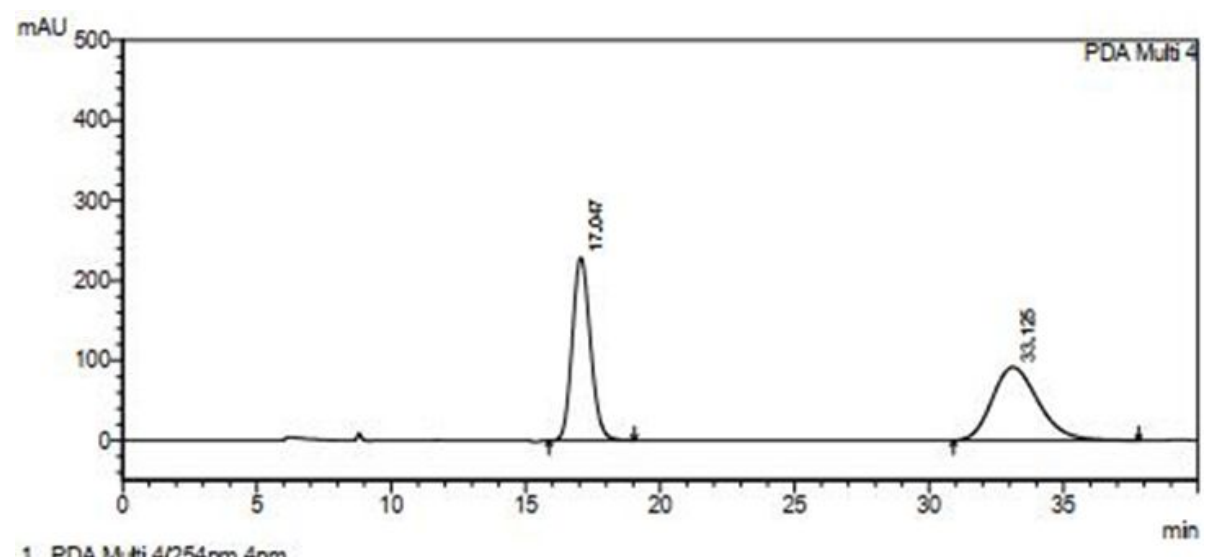

1 PDA Muti $4 / 254 \mathrm{~mm} 4 \mathrm{~nm}$

PeakTable
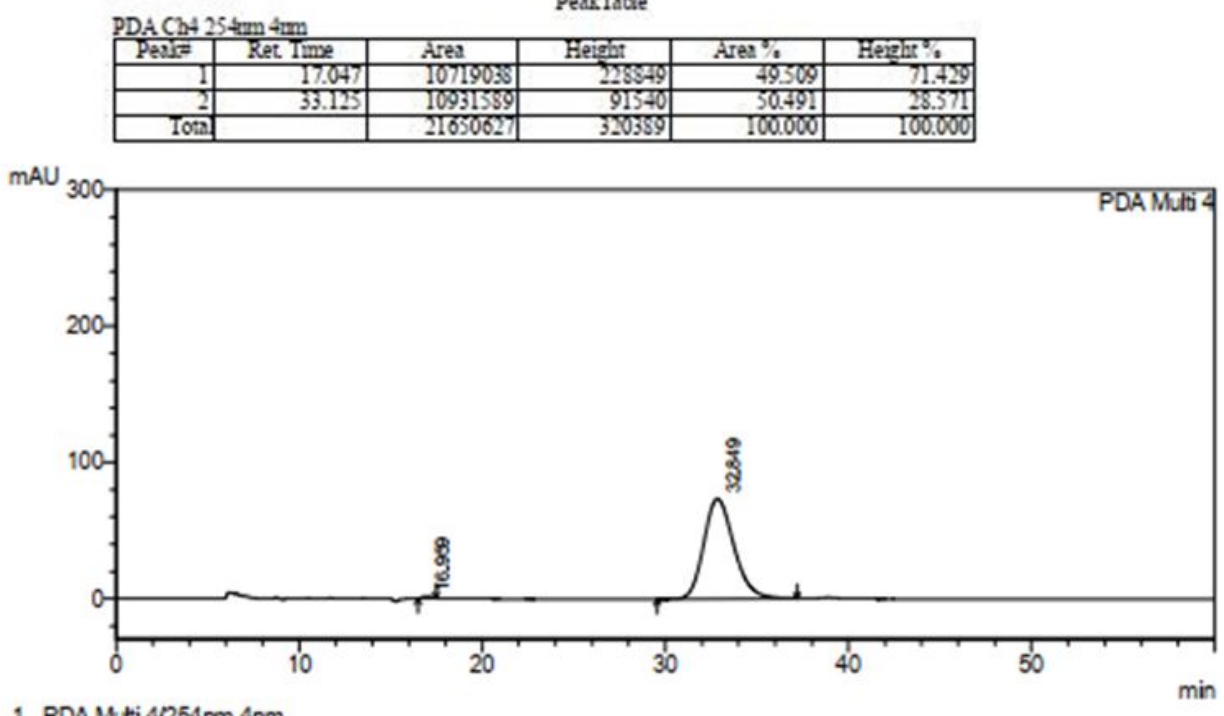

PevikTable

\begin{tabular}{|c|c|c|c|c|c|}
\hline & & 71702 & 752 & 0.207 & \\
\hline & & & & 0.491 & $\begin{array}{ll}1.079 \\
321\end{array}$ \\
\hline Tot & & 3405956 & 74624 & 100000 & 100000 \\
\hline
\end{tabular}

(S)-2-(3-Benzamido-2-oxo-1-phenylindolin-3-yl)ethane-1-sulfonyl fluoride (3b)<smiles>O=C(N[C@]1(CCS(=O)(=O)F)C(=O)N(c2ccccc2)c2ccccc21)c1ccccc1</smiles>

$3 \mathbf{b}$

Petroleum ether/ethyl acetate $=8: 1$ was used as the eluent for column chromatography. White solid (38.5 mg, 88\%), m. p. $183.6-184.3^{\circ} \mathrm{C} .{ }^{1} \mathbf{H}$ NMR $\left(500 \mathrm{MHz}, \mathrm{CDCl}_{3}\right) \delta$ 7.79-7.74 (m, 2H), 7.55-7.49 (m, 4H), 7.49-7.42 (m, 3H), 7.37 (t, $J=7.5 \mathrm{~Hz}, 2 \mathrm{H}), 7.32-7.25(\mathrm{~m}, 2 \mathrm{H}), 7.12-7.07(\mathrm{~m}, 1 \mathrm{H}), 6.83(\mathrm{~d}, J=8.0$ $\mathrm{Hz}, 1 \mathrm{H}), 3.86-3.77(\mathrm{~m}, 1 \mathrm{H}), 3.57-3.48(\mathrm{~m}, 1 \mathrm{H}), 2.87-2.78(\mathrm{~m}, 1 \mathrm{H}), 2.60-2.51(\mathrm{~m}, 1 \mathrm{H}) .{ }^{13} \mathbf{C}$ NMR $(126$ $\left.\mathrm{MHz}, \mathrm{CDCl}_{3}\right) \delta 174.68,166.91,143.60,134.14,132.33,132.30,129.84,128.69,128.66,128.04,127.41$, $126.90,123.93,123.04,110.13,59.92,44.99\left(\mathrm{~d}, J_{\mathrm{C}-\mathrm{F}}=18.1 \mathrm{~Hz}\right), 30.21 .{ }^{19} \mathbf{F} \mathbf{N M R}\left(471 \mathrm{MHz}, \mathrm{CDCl}_{3}\right) \delta$ 53.38. HRMS (ESI) $\mathrm{m} / z$ : Calcd. for $\mathrm{C}_{23} \mathrm{H}_{19} \mathrm{FN}_{2} \mathrm{O}_{4} \mathrm{SNa}$, [M+Na] $]^{+}$, 461.0942; found: 461.0946. $[\alpha]_{\mathrm{D}^{20}}=+$ $26.2\left(\mathrm{c} 1.0, \mathrm{CH}_{2} \mathrm{Cl}_{2}\right)$. Chiral HPLC analysis (Chiral pak AS-H, $i-\mathrm{PrOH} / n$-hexane $=20: 80$, flow rate $=$ 
$0.5 \mathrm{~mL} / \mathrm{min}$, wave length $=254 \mathrm{~nm}), \mathrm{t}_{\mathrm{R}}($ minor enantiomer $)=13.335 \mathrm{~min}, \mathrm{t}_{\mathrm{R}}($ major enantiomer $)=30.536$ $\min , 99 \%$ ee.

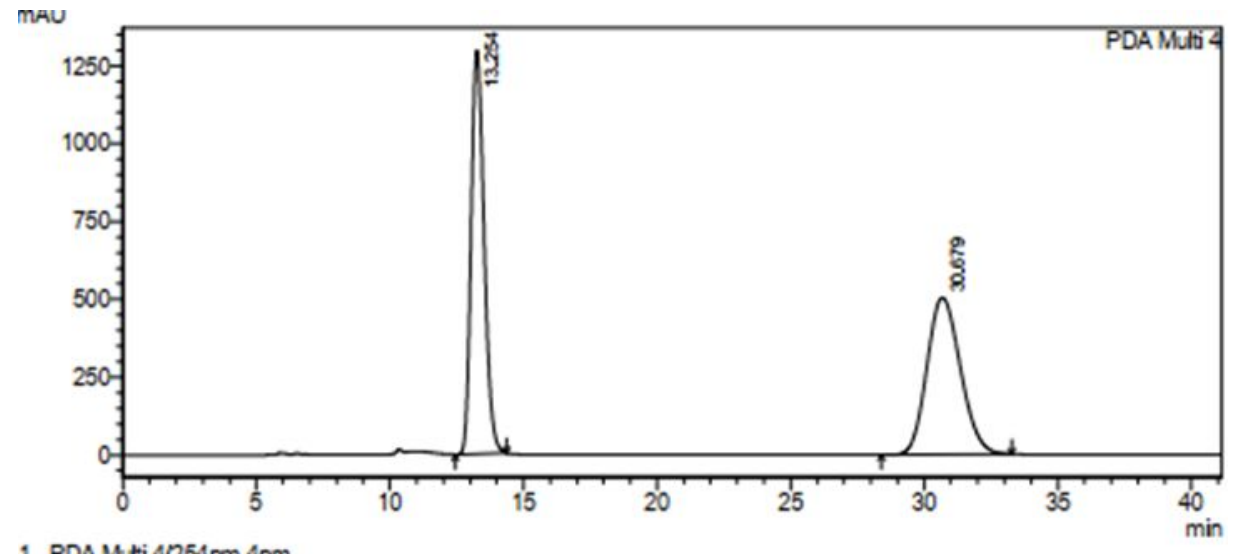

1 PDA Muti $4 / 254 \mathrm{~mm} 4 \mathrm{~nm}$
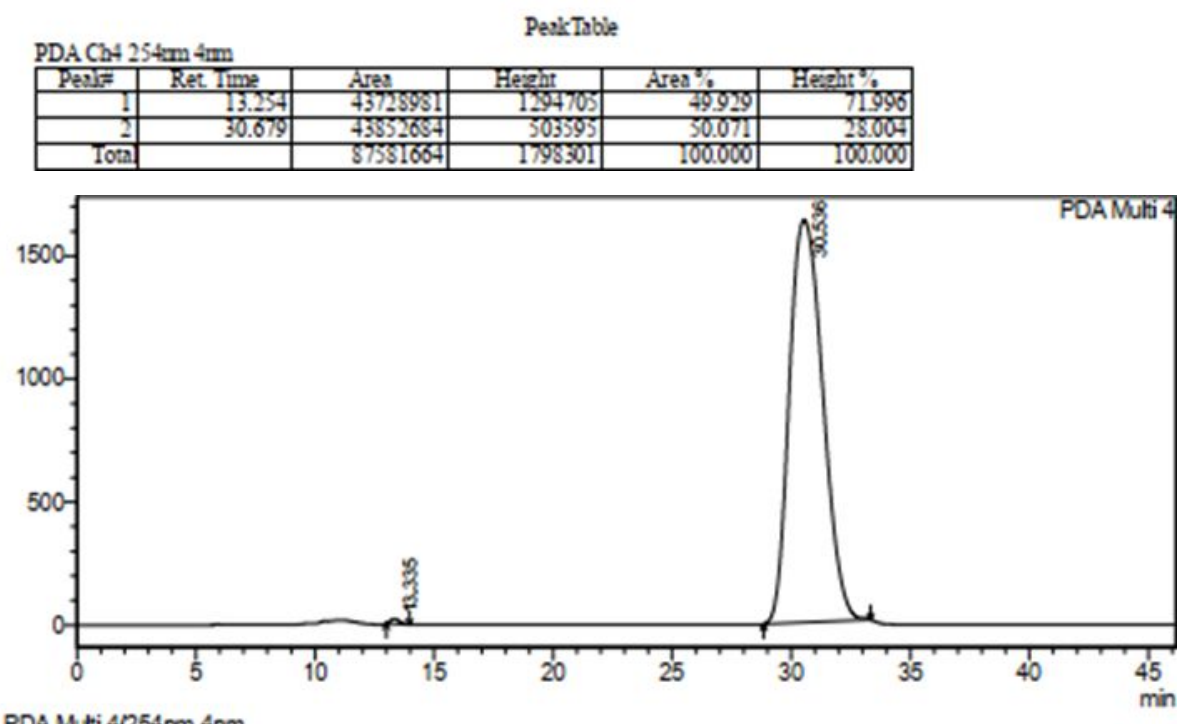

PDA Muti $4 / 254 \mathrm{~mm} 4 \mathrm{~nm}$

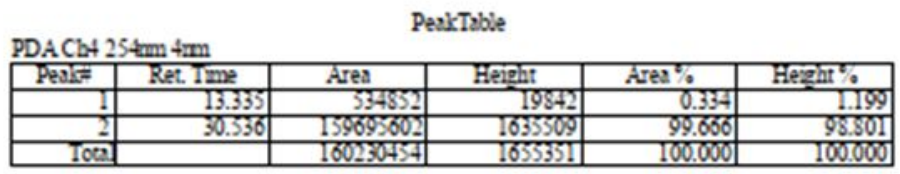

(S)-2-(3-Benzamido-1-methyl-2-oxoindolin-3-yl)ethane-1-sulfonyl fluoride (3c)<smiles>CN1C(=O)C(CCS(=O)(=O)F)(NC(=O)c2ccccc2)c2ccccc21</smiles>

Petroleum ether/ethyl acetate $=8: 1$ was used as the eluent for column chromatography. White solid (32 mg, $85 \%$ ), m. p. $187.3-188.9^{\circ} \mathrm{C} .{ }^{1} \mathbf{H}$ NMR $\left(400 \mathrm{MHz}, \mathrm{CDCl}_{3}\right) \delta 7.77(\mathrm{~d}, J=7.6 \mathrm{~Hz}, 2 \mathrm{H}), 7.55-7.35(\mathrm{~m}$, $5 \mathrm{H}), 7.16-7.09(\mathrm{~m}, 1 \mathrm{H}), 7.04(\mathrm{~s}, 1 \mathrm{H}), 6.94(\mathrm{~d}, J=8.0 \mathrm{~Hz}, 1 \mathrm{H}), 3.76(\mathrm{ddd}, J=15.6,10.8,6.0 \mathrm{~Hz}, 1 \mathrm{H})$, 3.54-3.44 (m, 1H), 3.32 (s, 3H), $2.88(\mathrm{ddd}, J=14.4,9.6,5.2 \mathrm{~Hz}, 1 \mathrm{H}), 2.49-2.38(\mathrm{~m}, 1 \mathrm{H}) .{ }^{13} \mathbf{C}$ NMR $(101$ $\left.\mathrm{MHz}, \mathrm{CDCl}_{3}\right) \delta 174.86,166.87,143.19,132.43,132.31,130.01,128.67,128.34,127.34,123.61,123.20$, 108.93, 59.91, $45.04\left(\mathrm{~d}, J_{\mathrm{C}-\mathrm{F}}=18.0 \mathrm{~Hz}\right), 29.96,26.80 .{ }^{19} \mathbf{F}$ NMR $\left(376 \mathrm{MHz}, \mathrm{CDCl}_{3}\right) \delta 53.16 . \mathbf{H R M S}$ 
(ESI) $\mathrm{m} / \mathrm{z}$ : Calcd. for $\mathrm{C}_{18} \mathrm{H}_{17} \mathrm{~N}_{2} \mathrm{O}_{4} \mathrm{FSNa},[\mathrm{M}+\mathrm{Na}]^{+}$, 399.0785; found: 399.0787. $[\alpha]_{\mathrm{D}}{ }^{20}=+32.5$ (c 1.0, $\mathrm{CH}_{2} \mathrm{Cl}_{2}$ ). Chiral HPLC analysis (Chiral pak AS-H, $i-\mathrm{PrOH} / n$-hexane $=20: 80$, flow rate $=0.5 \mathrm{~mL} / \mathrm{min}$, wave length $=254 \mathrm{~nm}), t_{R}($ minor enantiomer $)=13.405 \mathrm{~min}, t_{R}($ major enantiomer $)=30.599 \min , 99 \%$ ee.

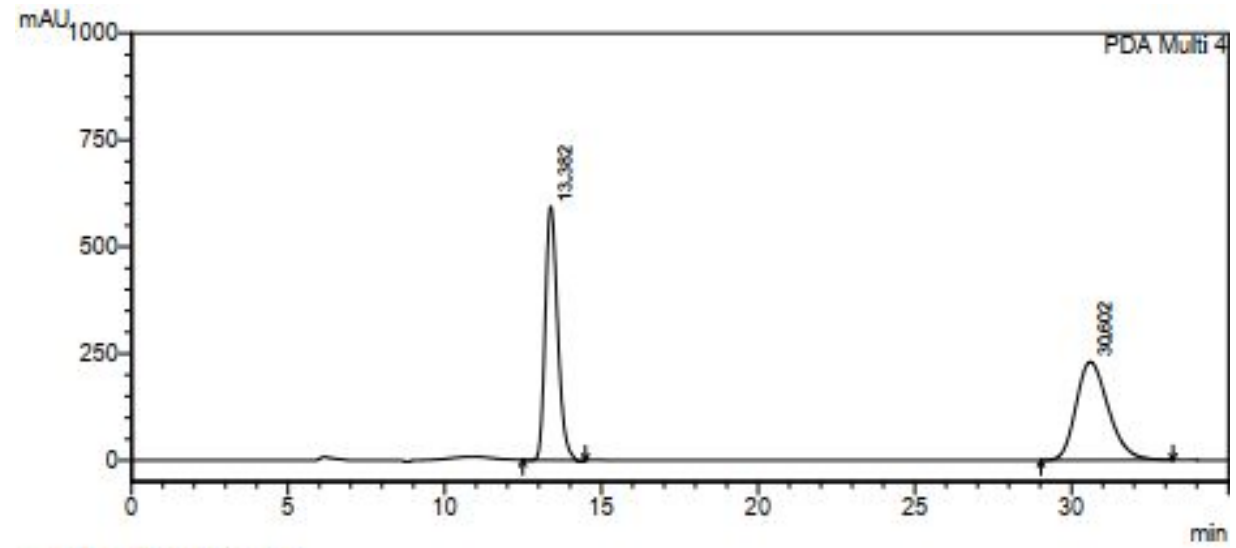

1 PDA Multi 4/254nm 4nm

PeakTable

PDA Cb4 254 am Anm
\begin{tabular}{|r|r|r|r|r|r|}
\hline Pealk & Rec. Time & Area & Height & Area \% & Height \% \\
\hline 1 & 13.382 & 16271949 & 594225 & 49.685 & 72.098 \\
\hline 2 & 30.002 & 16478272 & 229971 & 50.315 & 27.902 \\
\hline Tocal & & 32750221 & 824196 & 100.000 & 100.000 \\
\hline
\end{tabular}
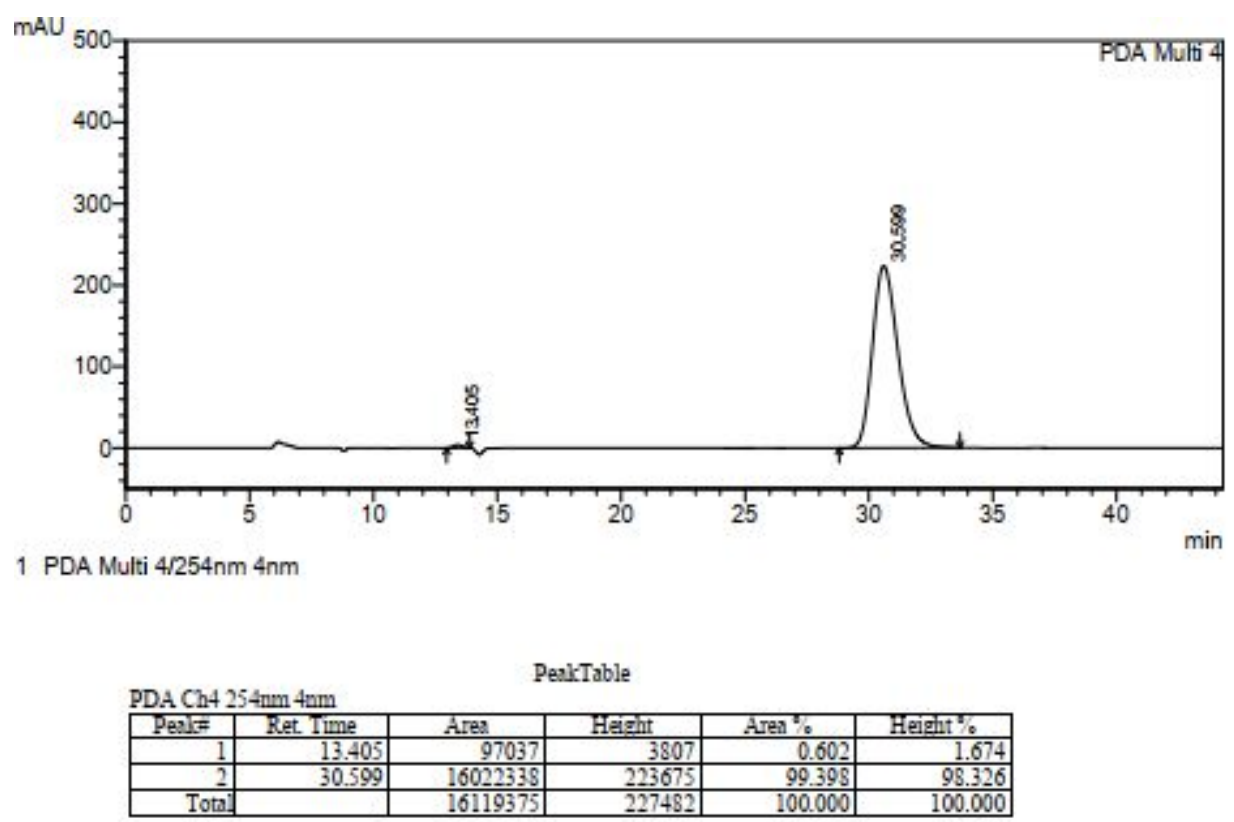

(S)-2-(3-Benzamido-1-benzyl-5-fluoro-2-oxoindolin-3-yl)ethane-1-sulfonyl fluoride (3d)<smiles>O=C(N[C@]1(CCS(=O)(=O)F)C(=O)N(Cc2ccccc2)c2ccc(F)cc21)c1ccccc1</smiles>

$3 d$ 
Petroleum ether/ethyl acetate $=8: 1$ was used as the eluent for column chromatography. White solid (45.6 mg, 97\%), m. p. $227.5-228.5^{\circ} \mathrm{C}^{1}{ }^{1} \mathbf{H}$ NMR (400 MHz, $\left.\mathrm{CDCl}_{3}\right) \delta 7.79-7.71(\mathrm{~m}, 2 \mathrm{H}), 7.51(\mathrm{t}, J=7.6$ $\mathrm{Hz}, 1 \mathrm{H}), 7.43-7.27(\mathrm{~m}, 8 \mathrm{H}), 7.14(\mathrm{dd}, J=7.6,2.8 \mathrm{~Hz}, 1 \mathrm{H}), 6.92(\mathrm{td}, J=8.8,2.8 \mathrm{~Hz}, 1 \mathrm{H}), 6.70(\mathrm{dd}, J=$ 8.4, 4.0 Hz, 1H), 5.02-4.93 (m, 2H), 3.77-3.67 (m, 1H), 3.53-3.42 (m, 1H), 2.87-2.76 (m, 1H), 2.52-2.41 $(\mathrm{m}, 1 \mathrm{H}) .{ }^{13} \mathrm{C}$ NMR $\left(101 \mathrm{MHz}, \mathrm{CDCl}_{3}\right) \delta 174.91,166.86,159.62\left(\mathrm{~d}, J_{\mathrm{C}-\mathrm{F}}=243.2 \mathrm{~Hz}\right), 138.06\left(\mathrm{~d}, J_{\mathrm{C}-\mathrm{F}}=\right.$ $2.1 \mathrm{~Hz}), 134.94,132.46,132.09,130.05\left(\mathrm{~d}, J_{\mathrm{C}-\mathrm{F}}=7.8 \mathrm{~Hz}\right), 129.10,128.73,128.05,127.38,127.28$, $116.16\left(\mathrm{~d}, J_{\mathrm{C}-\mathrm{F}}=23.5 \mathrm{~Hz}\right), 111.35\left(\mathrm{~d}, J_{\mathrm{C}-\mathrm{F}}=25.3 \mathrm{~Hz}\right), 110.81\left(\mathrm{~d}, J_{\mathrm{C}-\mathrm{F}}=8.0 \mathrm{~Hz}\right), 60.12,44.82\left(\mathrm{~d}, J_{\mathrm{C}-\mathrm{F}}=\right.$ 18.3 Hz), 44.57, 30.27. ${ }^{19}$ F NMR (376 $\left.\mathrm{MHz} \mathrm{CDCl}_{3}\right) \delta 53.40,-118.35$. HRMS (ESI) $\mathrm{m} / \mathrm{z}$ : Calcd. for

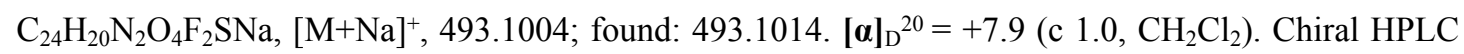
analysis (Chiral pak AS-H, $i-\mathrm{PrOH} / n$-hexane $=20: 80$, flow rate $=0.5 \mathrm{~mL} / \mathrm{min}$, wave length $=254 \mathrm{~nm}$ ), $\mathrm{t}_{\mathrm{R}}($ minor enantiomer $)=14.508 \mathrm{~min}, \mathrm{t}_{\mathrm{R}}($ major enantiomer $)=27.752 \mathrm{~min}, 98 \%$ ee.

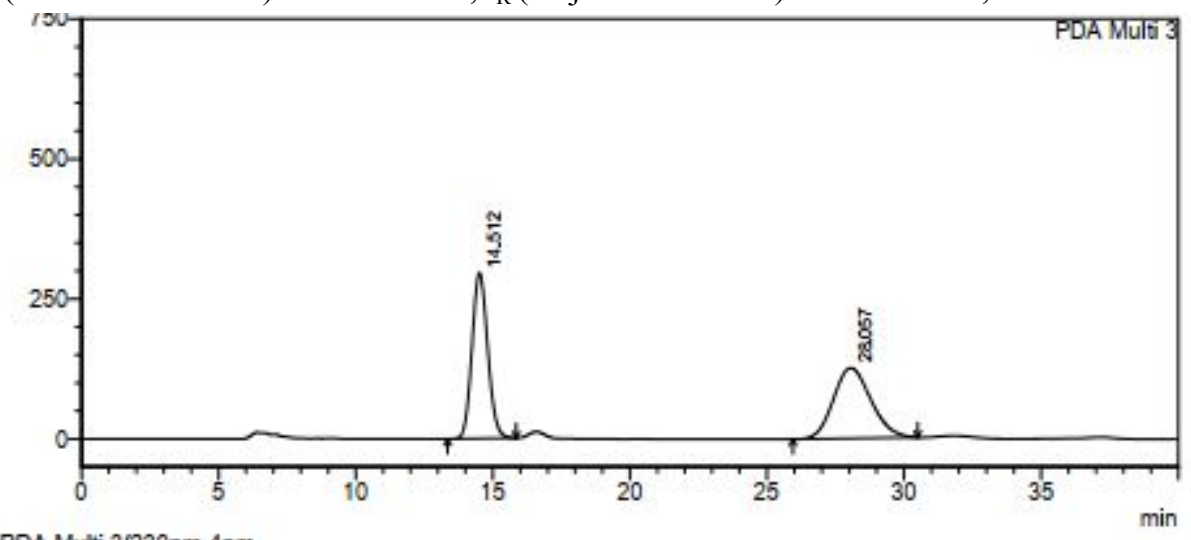

PDA Multi 3/230nm 4nm

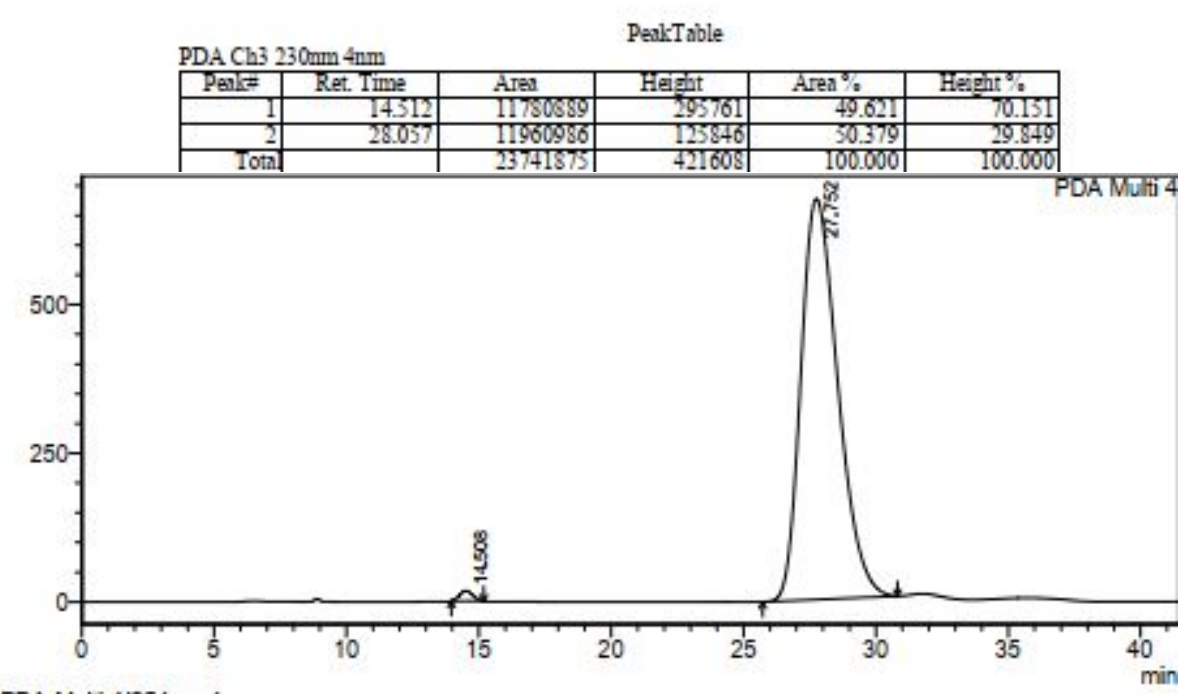

PDA Multi 4/254nm 4nm

PeakTable

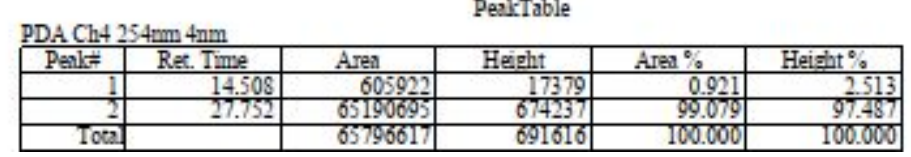

(S)-2-(3-Benzamido-1-benzyl-5-chloro-2-oxoindolin-3-yl)ethane-1-sulfonyl fluoride (3e) 
<smiles>O=C(N[C@]1(CCS(=O)(=O)F)C(=O)Nc2ccc(Cl)cc21)c1ccccc1</smiles>

Petroleum ether/ethyl acetate $=8: 1$ was used as the eluent for column chromatography. White solid (48.5 mg, 99\%), m. p. $246.7-247.5{ }^{\circ} \mathrm{C} .{ }^{1} \mathbf{H}$ NMR $\left(400 \mathrm{MHz}, \mathrm{CDCl}_{3}\right) \delta 7.75(\mathrm{~d}, J=7.6 \mathrm{~Hz}, 2 \mathrm{H}), 7.54-$ $7.48(\mathrm{~m}, 1 \mathrm{H}), 7.44-7.28(\mathrm{~m}, 9 \mathrm{H}), 7.20(\mathrm{~d}, J=8.0 \mathrm{~Hz}, 1 \mathrm{H}), 6.70(\mathrm{~d}, J=8.4 \mathrm{~Hz}, 1 \mathrm{H}), 4.97(\mathrm{~s}, 2 \mathrm{H}), 3.81-$ $3.69(\mathrm{~m}, 1 \mathrm{H}), 3.55-3.44(\mathrm{~m}, 1 \mathrm{H}), 2.85-2.73(\mathrm{~m}, 1 \mathrm{H}), 2.50-2.39(\mathrm{~m}, 1 \mathrm{H}) .{ }^{13} \mathbf{C} \mathbf{~ N M R}\left(101 \mathrm{MHz}, \mathrm{CDCl}_{3}\right) \delta$ 174.69, 166.82, 140.71, 134.83, 132.50, 132.01, 130.15, 129.78, 129.11, 128.75, 128.09, 127.41, 127.27, 123.41, 111.07, 59.83, 44.75 (d, $\left.J_{\mathrm{C}-\mathrm{F}}=18.2 \mathrm{~Hz}\right), 44.55,30.27 .{ }^{19} \mathbf{F}$ NMR $\left(376 \mathrm{MHz}, \mathrm{CDCl}_{3}\right) \delta 53.40$.

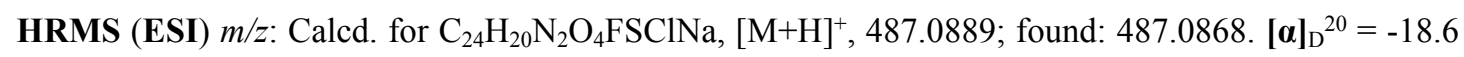
(c 1.0, $\mathrm{CH}_{2} \mathrm{Cl}_{2}$ ). Chiral HPLC analysis (Chiral pak AS-H, $i-\mathrm{PrOH} / n$-hexane $=20: 80$, flow rate $=0.5$ $\mathrm{mL} / \mathrm{min}$, wave length $=230 \mathrm{~nm}), \mathrm{t}_{\mathrm{R}}($ minor enantiomer $)=13.165 \mathrm{~min}, \mathrm{t}_{\mathrm{R}}($ major enantiomer $)=25.218$ $\min , 98 \% e e$.

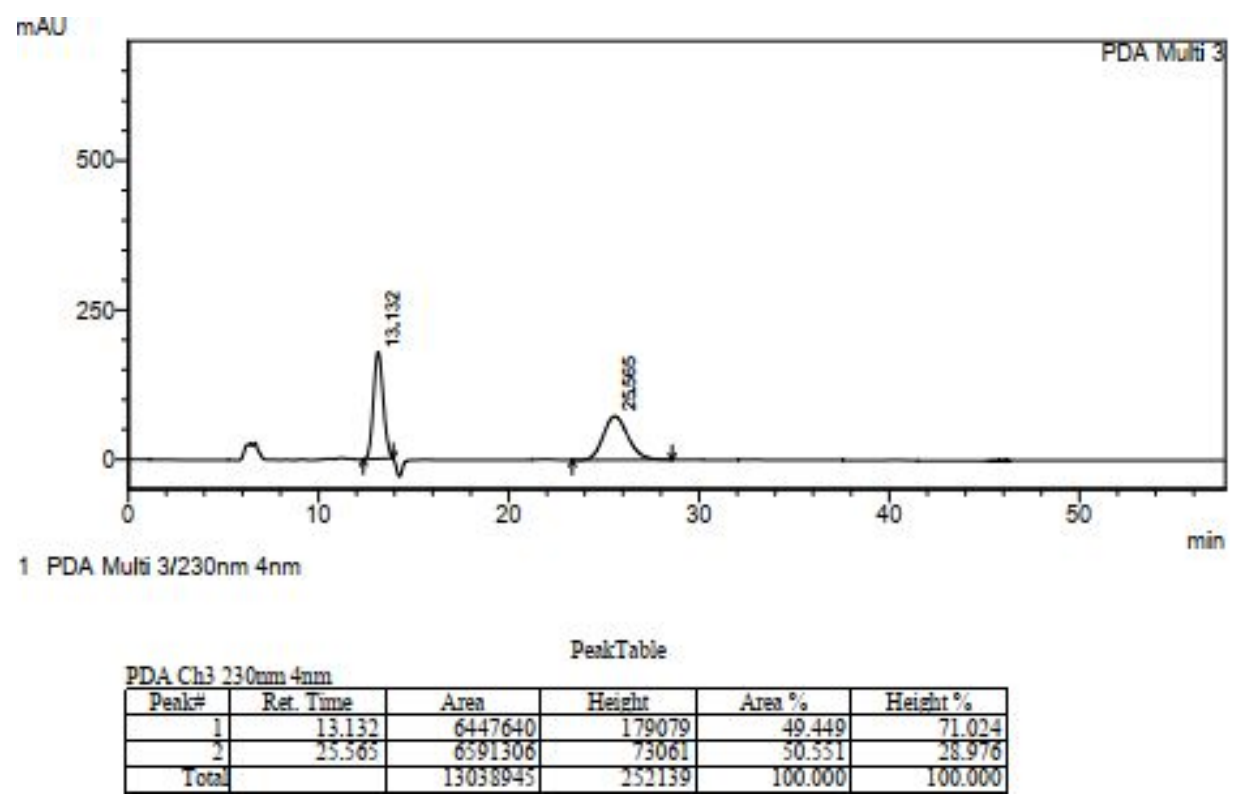




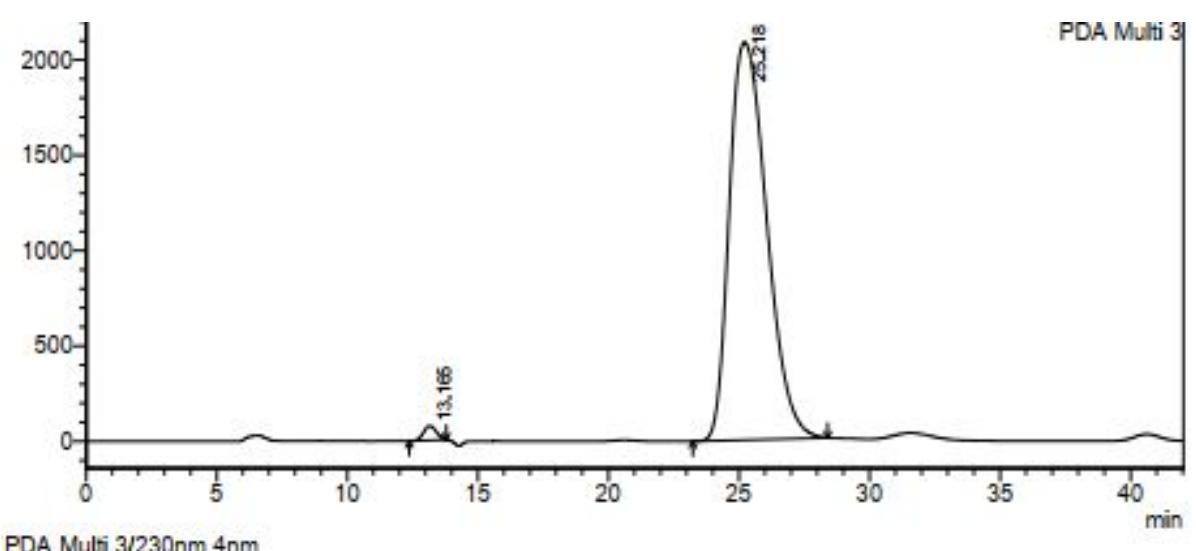

PDA Multi $3 / 230 \mathrm{~nm} 4 \mathrm{~nm}$

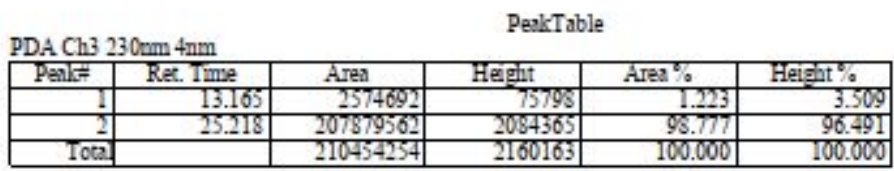

(S)-2-(3-Benzamido-1-benzyl-5-bromo-2-oxoindolin-3-yl)ethane-1-sulfonyl fluoride (3f)<smiles>O=C1N(Cc2ccccc2)c2ccc(Br)cc2[C@]1(CCS(=O)(=O)F)NC(=O)P(=O)(F)F</smiles>

Petroleum ether/ethyl acetate $=8: 1$ was used as the eluent for column chromatography. White solid (51.6 mg, 98\%), m. p. $201.8-203.4^{\circ} \mathrm{C} .{ }^{1} \mathbf{H}$ NMR $\left(500 \mathrm{MHz}, \mathrm{CDCl}_{3}\right) \delta 7.79(\mathrm{~d}, J=7.5 \mathrm{~Hz}, 2 \mathrm{H}), 7.57-$ $7.49(\mathrm{~m}, 2 \mathrm{H}), 7.43(\mathrm{t}, J=7.5 \mathrm{~Hz}, 2 \mathrm{H}), 7.41-7.33(\mathrm{~m}, 5 \mathrm{H}), 7.33-7.28(\mathrm{~m}, 1 \mathrm{H}), 7.23(\mathrm{~s}, 1 \mathrm{H}), 6.66(\mathrm{~d}, J=$ $8.5 \mathrm{~Hz}, 1 \mathrm{H}), 4.99$ (s, $2 \mathrm{H}), 3.87-3.78(\mathrm{~m}, 1 \mathrm{H}), 3.56-3.47$ (m, 1H), 2.83 (ddd, $J=14.5,9.0,5.5 \mathrm{~Hz}, 1 \mathrm{H}$ ), 2.42 (ddd, $J=15.0,9.5,6.0 \mathrm{~Hz}, 1 \mathrm{H}) .{ }^{13} \mathbf{C}$ NMR $\left(126 \mathrm{MHz}, \mathrm{CDCl}_{3}\right) \delta 174.43,166.74,141.19,134.79$, $132.67,132.52,132.04,130.52,129.09,128.75,128.06,127.36,127.25,126.17,116.31,111.49,59.60$, $44.67\left(\mathrm{~d}, J_{\mathrm{C}-\mathrm{F}}=18.3 \mathrm{~Hz}\right), 44.50,30.24 .{ }^{19} \mathbf{F}$ NMR $\left(471 \mathrm{MHz}, \mathrm{CDCl}_{3}\right) \delta 53.33$. HRMS (ESI) $\mathrm{m} / z$ : Calcd. for $\mathrm{C}_{24} \mathrm{H}_{20} \mathrm{~N}_{2} \mathrm{O}_{4} \mathrm{FSBrNa}$, [M+Na] $]^{+}$, 553.0203; found: 553.0206. $[\alpha]_{\mathrm{D}}{ }^{20}=-14.0\left(\mathrm{c} 0.5, \mathrm{CH}_{2} \mathrm{Cl}_{2}\right)$. Chiral HPLC analysis (Chiral pak AS-H, $i-\mathrm{PrOH} / n$-hexane $=20: 80$, flow rate $=0.5 \mathrm{~mL} / \mathrm{min}$, wave length $=$ $254 \mathrm{~nm}), \mathrm{t}_{\mathrm{R}}($ minor enantiomer $)=14.052 \mathrm{~min}, \mathrm{t}_{\mathrm{R}}($ major enantiomer $)=28.872 \mathrm{~min}, 99 \% \mathrm{ee}$. 


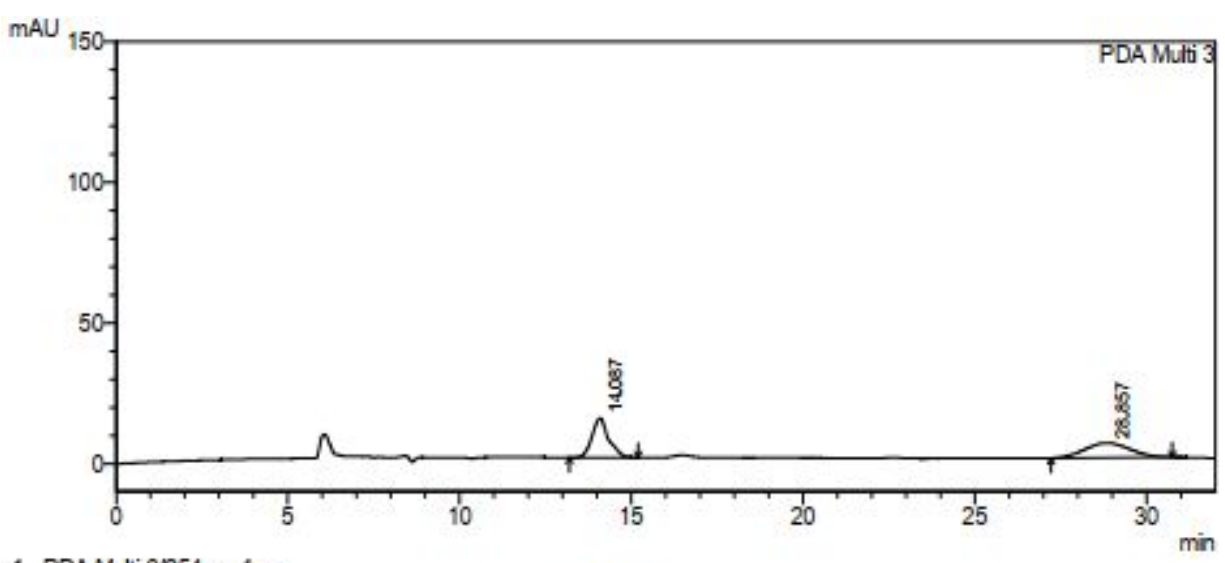

1. PDA Muti $3 / 254 \mathrm{rm} 4 \mathrm{~nm}$
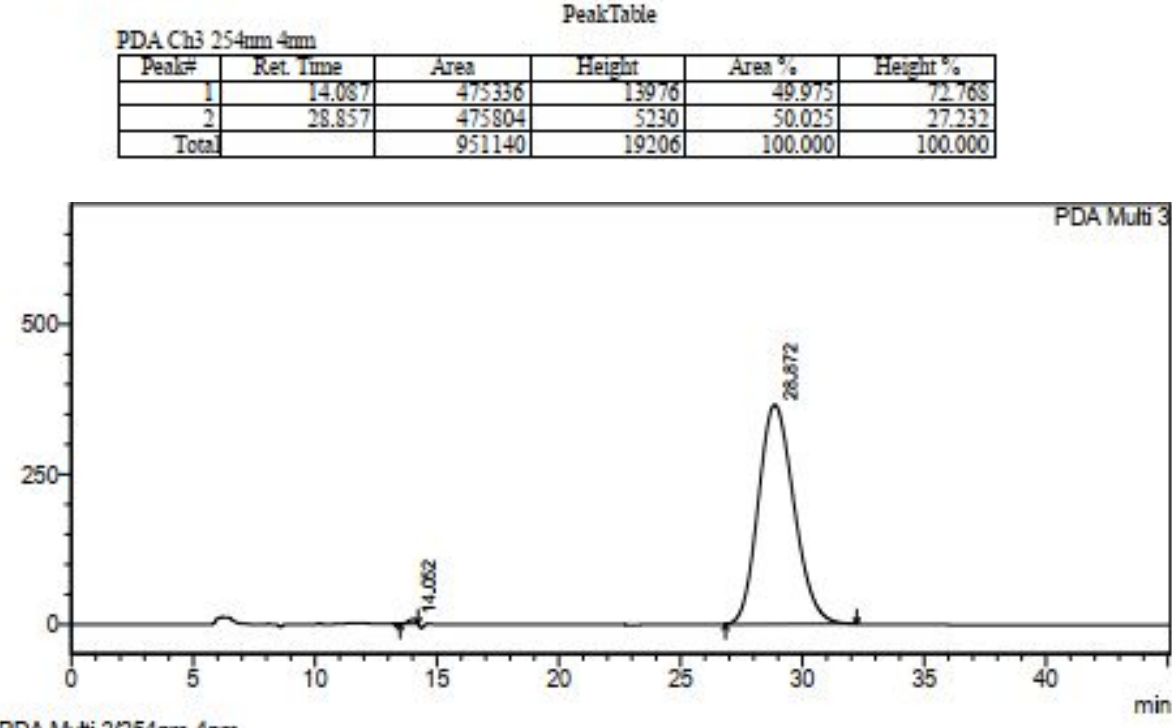

PDA Mutti $3 / 254 \mathrm{~mm} 4 \mathrm{~nm}$

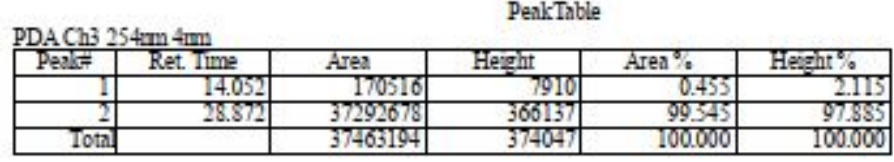

(S)-2-(3- Benzamido-1-benzyl-5-methyl-2-oxoindolin-3-yl)ethane-1-sulfonyl fluoride (3g)<smiles>Cc1ccc2c(c1)[C@](CCS(=O)(=O)F)(CCS(=O)(=O)NC(=O)O)C(=O)N2Cc1ccccc1</smiles>

Petroleum ether/ethyl acetate $=8: 1$ was used as the eluent for column chromatography. White solid (45 mg, 98\%), m. p. $221.5-223.1^{\circ} \mathrm{C} .{ }^{1} \mathbf{H}$ NMR (400 MHz, $\left.\mathrm{CDCl}_{3}\right) \delta$ 7.83-7.76 (m, 2H), 7.56-7.49 (m, $\left.1 \mathrm{H}\right)$, 7. $45-7.39(\mathrm{~m}, 4 \mathrm{H}), 7.39-7.34(\mathrm{~m}, 2 \mathrm{H}), 7.32-7.27(\mathrm{~m}, 1 \mathrm{H}), 7.24(\mathrm{~s}, 1 \mathrm{H}), 7.12(\mathrm{~s}, 1 \mathrm{H}), 7.03(\mathrm{dd}, J=8.0$, $0.8 \mathrm{~Hz}, 1 \mathrm{H}), 6.68(\mathrm{~d}, J=8.0 \mathrm{~Hz}, 1 \mathrm{H}), 5.06-4.92(\mathrm{~m}, 2 \mathrm{H}), 3.79-3.65(\mathrm{~m}, 1 \mathrm{H}), 3.52-3.41(\mathrm{~m}, 1 \mathrm{H}), 2.86$ (ddd, $J=14.4,9.6,5.2 \mathrm{~Hz}, 1 \mathrm{H}), 2.47$ (ddd, $J=14.4,10.0,5.6 \mathrm{~Hz}, 1 \mathrm{H}), 2.28(\mathrm{~s}, 3 \mathrm{H}) .{ }^{13} \mathrm{C}$ NMR $(101$ $\left.\mathrm{MHz}, \mathrm{CDCl}_{3}\right) \delta 174.80,166.66,139.74,135.38,133.35,132.49,132.29,130.17,128.96,128.67,128.31$, $127.85,127.33,127.30,123.98,109.76,59.95,44.97\left(\mathrm{~d}, J_{\mathrm{C}-\mathrm{F}}=18.1 \mathrm{~Hz}\right), 44.41,30.44,21.12 .{ }^{19} \mathbf{F}$ NMR $\left(376 \mathrm{MHz}, \mathrm{CDCl}_{3}\right) \delta$ 53.19. HRMS (ESI) $m / z$ : Calcd. for $\mathrm{C}_{25} \mathrm{H}_{23} \mathrm{~N}_{2} \mathrm{O}_{4} \mathrm{FSNa}$, [M+Na] $]^{+}$, 489.1255; found: 
489.1271. $[\alpha]_{\mathrm{D}}{ }^{20}=-4.4\left(\mathrm{c} 0.5, \mathrm{CH}_{2} \mathrm{Cl}_{2}\right)$. Chiral HPLC analysis (Chiral pak AS-H, $i-\mathrm{PrOH} / n$-hexane $=20$ : 80 , flow rate $=0.5 \mathrm{~mL} / \mathrm{min}$, wave length $=254 \mathrm{~nm}), \mathrm{t}_{\mathrm{R}}($ minor enantiomer $)=14.919 \mathrm{~min}, \mathrm{t}_{\mathrm{R}}($ major enantiomer) $=27.823 \mathrm{~min}, 99 \%$ ee

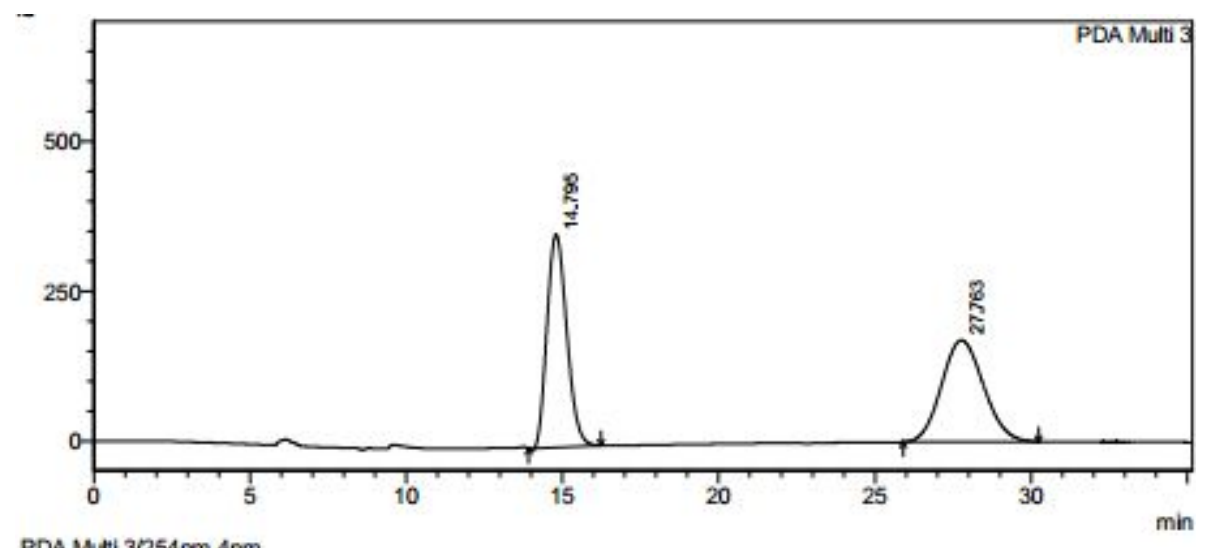

PDA Multi $3 / 254 \mathrm{~mm} 4 \mathrm{~nm}$

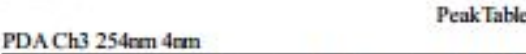

\begin{tabular}{|r|r|r|r|r|r|}
\hline \multicolumn{1}{|c|}{ Peakiki } & Ret. Time & \multicolumn{1}{|c|}{ Area } & Heisht & Area\% & \multicolumn{1}{|c|}{ Height \% } \\
\hline 1 & 14.795 & 15616064 & 3558.30 & 49.635 & 67.729 \\
\hline 2 & 27.763 & 15845765 & 169541 & 50.365 & 32.271 \\
\hline Total & & 31461830 & 525370 & 100.000 & 100.000 \\
\hline
\end{tabular}

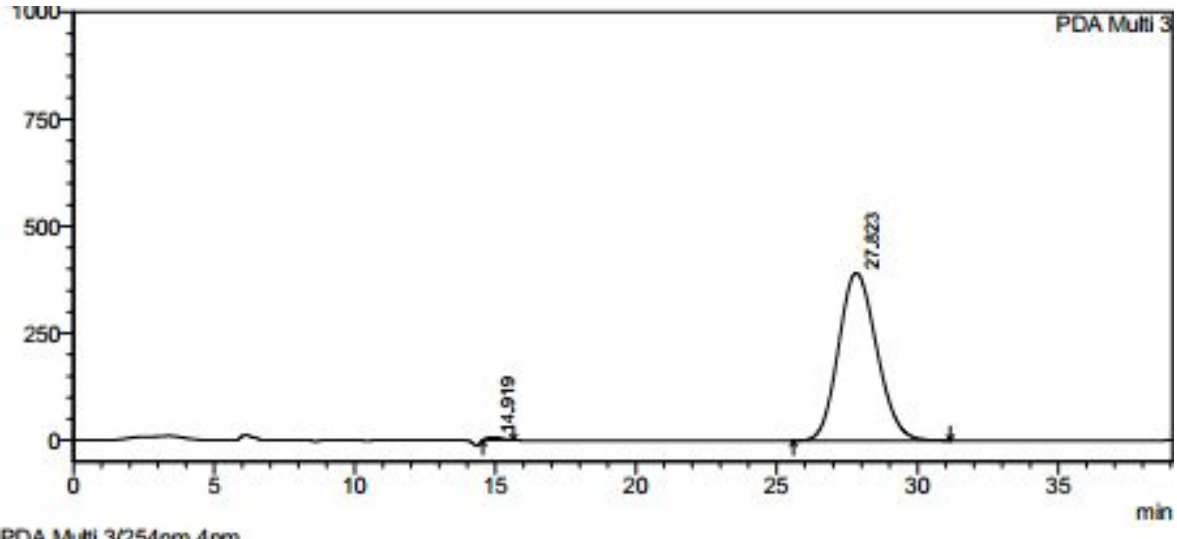

PDA Mutii 3/254nm 4m

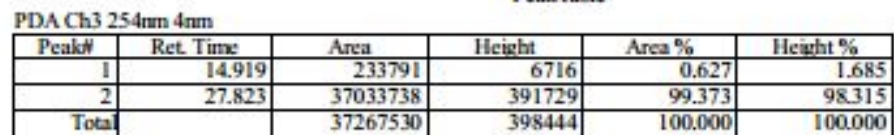

(S)-2-(3-Benzamido-1-benzyl-5-methoxy-2-oxoindolin-3-yl)ethane-1-sulfonyl fluoride (3h)<smiles>COc1ccc2c(c1)[C@](CCS(=O)(=O)F)(NC(=O)c1ccccc1)C(=O)N2Cc1ccccc1</smiles>

3h

Petroleum ether/ethyl acetate $=8: 1$ was used as the eluent for column chromatography. White solid (47.5 mg, 99\%), m. p. 204.6-205.6 ${ }^{\circ} \mathrm{C} .{ }^{1} \mathbf{H}$ NMR (400 MHz, $\left.\mathrm{CDCl}_{3}\right) \delta$ 7.81-7.77 (m, 2H), 7.56-7.50 $(\mathrm{m}, 1 \mathrm{H}), 7.46-7.36(\mathrm{~m}, 7 \mathrm{H}), 7.34-7.30(\mathrm{~m}, 1 \mathrm{H}), 7.23(\mathrm{~s}, 1 \mathrm{H}), 7.07(\mathrm{~d}, J=2.4 \mathrm{~Hz}, 1 \mathrm{H}), 6.77(\mathrm{dd}, J=8.8$, $2.4 \mathrm{~Hz}, 1 \mathrm{H}), 6.72(\mathrm{~d}, J=8.4 \mathrm{~Hz}, 1 \mathrm{H}), 5.00(\mathrm{~s}, 2 \mathrm{H}), 3.75(\mathrm{~s}, 3 \mathrm{H}), 3.73-3.67(\mathrm{~m}, 1 \mathrm{H}), 3.53-3.43(\mathrm{~m}, 1 \mathrm{H})$, 2.93-2.83 (m, 1H), 2.56-2.47 (m, 1H). ${ }^{13} \mathbf{C}$ NMR (101 MHz, $\left.\mathrm{CDCl}_{3}\right) \delta 174.65,166.74,156.64,135.41$, 135.34, 132.45, 132.27, 129.66, 128.98, 128.65, 127.87, 127.34, 127.31, 113.98, 110.66, 110.52, 60.30, 
55.75, $45.00\left(\mathrm{~d}, J_{\mathrm{C}-\mathrm{F}}=18.3 \mathrm{~Hz}\right), 44.50,30.45 .{ }^{19} \mathbf{F}$ NMR $\left(471 \mathrm{MHz}, \mathrm{CDCl}_{3}\right) \delta 53.30$. HRMS (ESI) $\mathrm{m} / \mathrm{z}$ :

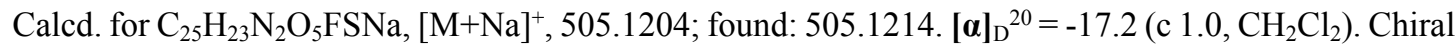
HPLC analysis (Chiral pak AS-H, $i$-PrOH $/ n$-hexane $=20: 80$, flow rate $=0.5 \mathrm{~mL} / \mathrm{min}$, wave length $=$ $254 \mathrm{~nm}), \mathrm{t}_{\mathrm{R}}($ minor enantiomer $)=8.296 \mathrm{~min}, \mathrm{t}_{\mathrm{R}}($ major enantiomer $)=23.530 \mathrm{~min}, 98 \%$ ee .

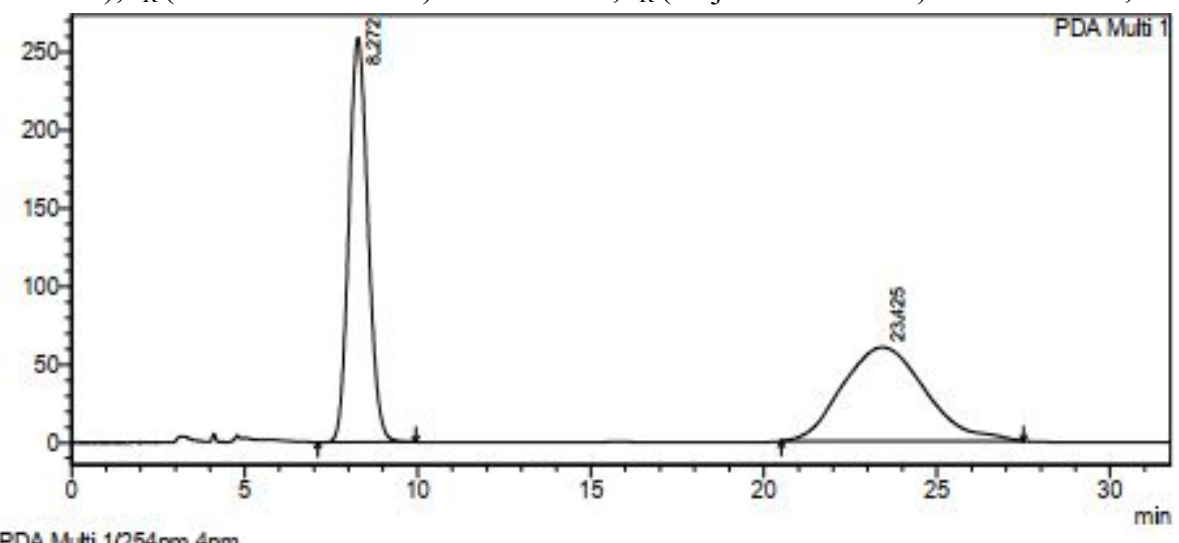

PDA Muti $1 / 254 \mathrm{~mm} 4 \mathrm{~nm}$
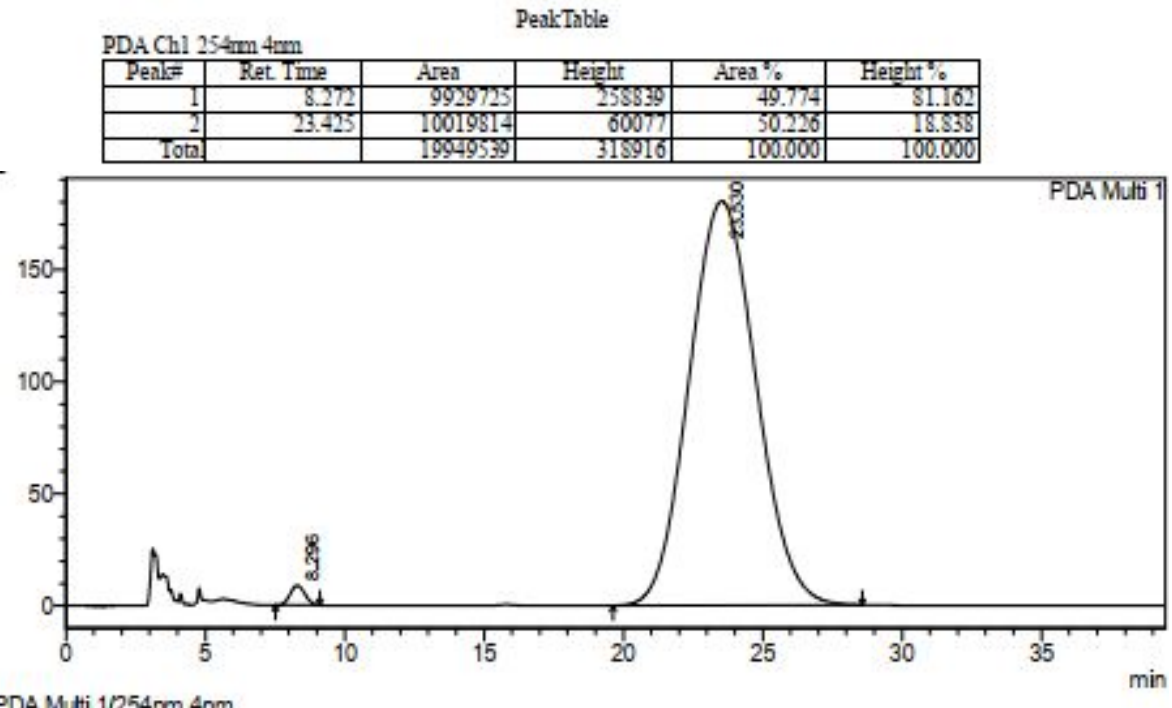

PDA Muti $1 / 254 \mathrm{rm} 4 \mathrm{~nm}$

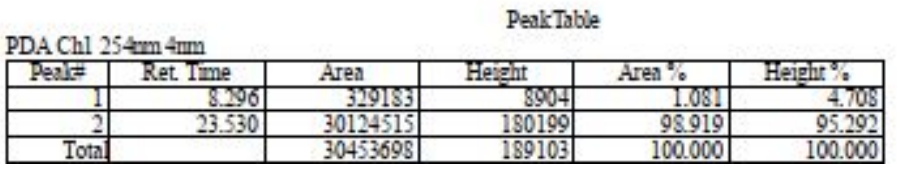

(S)-2-(3-Benzamido-1-benzyl-6-fluoro-2-oxoindolin-3-yl)ethane-1-sulfonyl fluoride (3i)<smiles>O=C(N[C@]1(CCS(=O)(=O)F)C(=O)N(Cc2ccccc2)c2cc(F)ccc21)c1ccccc1</smiles>

$3 i$

Petroleum ether/ethyl acetate $=8: 1$ was used as the eluent for column chromatography. White solid (42.6 mg, 91\%), m. p. 241.5-242.2 ${ }^{\circ} \mathrm{C} .{ }^{1} \mathbf{H}$ NMR (400 MHz, $\left.\mathrm{CDCl}_{3}\right) \delta$ 7.81-7.74 (m, 2H), 7.55-7.50 (m, 1H), 7.44-7.29 (m, 8H), 7.27 (s, 1H), 6.73 (ddd, $J=9.2,8.4,2.4 \mathrm{~Hz}, 1 \mathrm{H}), 6.60-6.50$ (m, 1H), 5.00 (d, $J$

$=16.0 \mathrm{~Hz}, 1 \mathrm{H}), 4.93(\mathrm{~d}, J=15.6 \mathrm{~Hz}, 1 \mathrm{H}), 3.79-3.68(\mathrm{~m}, 1 \mathrm{H}), 3.54-3.43(\mathrm{~m}, 1 \mathrm{H}), 2.82(\mathrm{ddd}, J=14.8$, 
10.0, 5.6 Hz, 1H), 2.51-2.40 (m, 1H). ${ }^{13} \mathbf{C}$ NMR $\left(101 \mathrm{MHz}, \mathrm{CDCl}_{3}\right) \delta 175.27,166.79,163.67\left(\mathrm{~d}, J_{\mathrm{C}-\mathrm{F}}=\right.$ $247.8 \mathrm{~Hz}), 143.87\left(\mathrm{~d}, J_{\mathrm{C}-\mathrm{F}}=11.7 \mathrm{~Hz}\right), 134.76,132.45,132.24,129.15,128.74,128.15,127.34,127.32$, $124.36\left(\mathrm{~d}, J_{\mathrm{C}-\mathrm{F}}=10.0 \mathrm{~Hz}\right), 123.80\left(\mathrm{~d}, J_{\mathrm{C}-\mathrm{F}}=2.9 \mathrm{~Hz}\right), 109.83\left(\mathrm{~d}, J_{\mathrm{C}-\mathrm{F}}=22.7 \mathrm{~Hz}\right), 98.96\left(\mathrm{~d}, J_{\mathrm{C}-\mathrm{F}}=27.9\right.$ $\mathrm{Hz}), 59.44,44.85$ (d, $\left.J_{\mathrm{C}-\mathrm{F}}=18.2 \mathrm{~Hz}\right), 44.62,30.35,29.72 .{ }^{19} \mathbf{F}$ NMR $\left(376 \mathrm{MHz}, \mathrm{CDCl}_{3}\right) \delta 53.34 .{ }^{19} \mathbf{F}$ NMR (376 MHz, $\left.\mathrm{CDCl}_{3}\right) \delta$-109.06. HRMS (ESI) $\mathrm{m} / z$ : Calcd. for $\mathrm{C}_{24} \mathrm{H}_{20} \mathrm{~N}_{2} \mathrm{O}_{4} \mathrm{~F}_{2} \mathrm{SNa},[\mathrm{M}+\mathrm{Na}]^{+}$, 493.1004; found: 493.1007. $[\alpha]_{\mathrm{D}}{ }^{20}=+11.4$ (c 1.0, $\mathrm{CH}_{2} \mathrm{Cl}_{2}$ ). Chiral HPLC analysis (Chiral pak AS-H, $i$ $\mathrm{PrOH} / n$-hexane $=20: 80$, flow rate $=0.5 \mathrm{~mL} / \mathrm{min}$, wave length $=254 \mathrm{~nm}), \mathrm{t}_{\mathrm{R}}($ minor enantiomer $)=$ $14.657 \mathrm{~min}, \mathrm{t}_{\mathrm{R}}$ (major enantiomer) $=22.642 \mathrm{~min}, 99 \%$ ee.

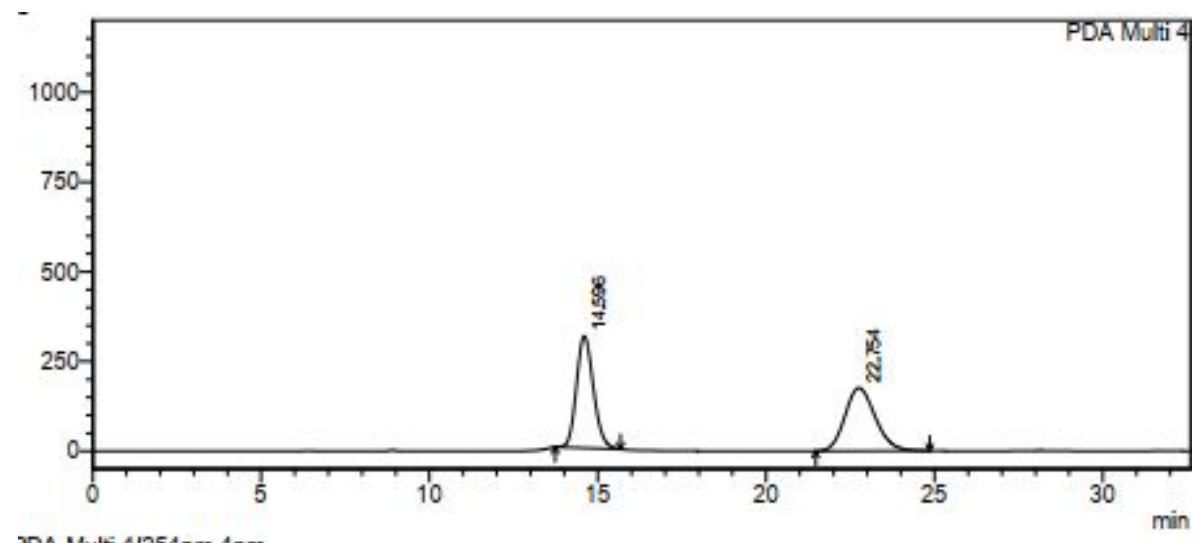

'DA Multi 4/254nm 4nm
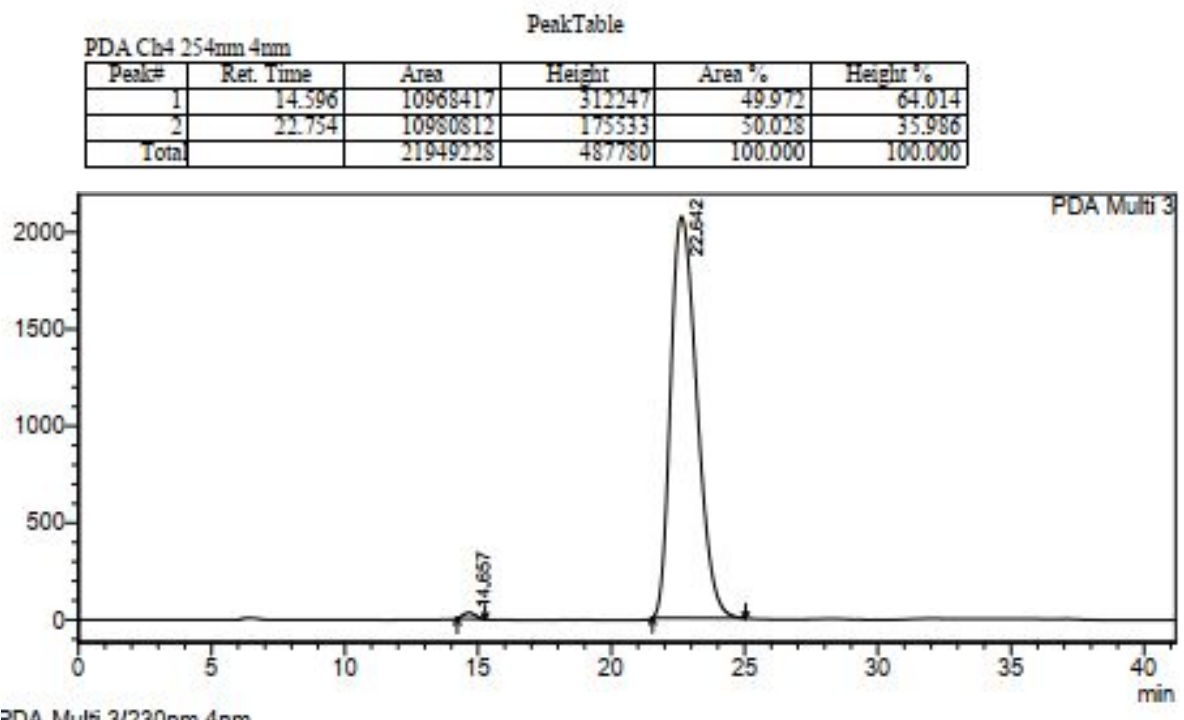

गDA Multi $3 / 230 \mathrm{~nm} 4 \mathrm{~nm}$

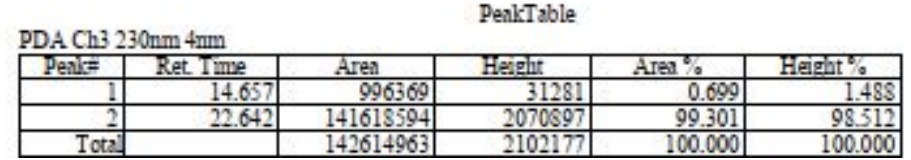

(S)-2-(3-Benzamido-1-benzyl-6-chloro-2-oxoindolin-3-yl)ethane-1-sulfonyl fluoride (3j)<smiles>O=C(N[C@]1(CCS(=O)(=O)F)C(=O)N(Cc2ccccc2)c2cc(Cl)ccc21)c1ccccc1</smiles> 
Petroleum ether/ethyl acetate $=8: 1$ was used as the eluent for column chromatography. White solid (44.8 mg, 92\%), m. p. 207.9-208.4 ${ }^{\circ} \mathrm{C} .{ }^{1} \mathbf{H}$ NMR (400 MHz, $\left.\mathrm{CDCl}_{3}\right) \delta 7.76(\mathrm{~d}, J=7.6 \mathrm{~Hz}, 2 \mathrm{H}), 7.54-$ $7.49(\mathrm{~m}, 1 \mathrm{H}), 7.45-7.26(\mathrm{~m}, 9 \mathrm{H}), 7.03(\mathrm{dd}, J=8.0,1.6 \mathrm{~Hz}, 1 \mathrm{H}), 6.79(\mathrm{~d}, J=1.2 \mathrm{~Hz}, 1 \mathrm{H}), 5.00(\mathrm{~d}, J=$ $15.6 \mathrm{~Hz}, 1 \mathrm{H}), 4.92(\mathrm{~d}, J=15.6 \mathrm{~Hz}, 1 \mathrm{H}), 3.80-3.67(\mathrm{~m}, 1 \mathrm{H}), 3.51-3.40$ (m, 1H), 2.79 (ddd, $J=14.4,9.6$, $5.2 \mathrm{~Hz}, 1 \mathrm{H}), 2.50-2.38(\mathrm{~m}, 1 \mathrm{H}) .{ }^{13} \mathbf{C} \mathbf{N M R}\left(101 \mathrm{MHz}, \mathrm{CDCl}_{3}\right) \delta 175.01,166.78,143.45,135.69,134.75$, $132.49,132.10,129.16,128.74,128.16,127.35,127.28,126.79,123.95,123.54,110.66,59.48,44.78$ $\left(\mathrm{d}, J_{\mathrm{C}-\mathrm{F}}=18.4 \mathrm{~Hz}\right), 44.56,30.22 .{ }^{19} \mathbf{F}$ NMR $\left(376 \mathrm{MHz}, \mathrm{CDCl}_{3}\right) \delta 53.39$. HRMS (ESI) $\mathrm{m} / \mathrm{z}$ : Calcd. for

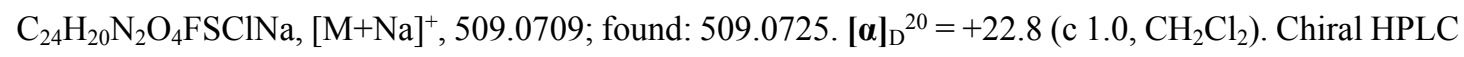
analysis (Chiral pak AS-H, $i-\mathrm{PrOH} / n$-hexane $=20: 80$, flow rate $=0.5 \mathrm{~mL} / \mathrm{min}$, wave length $=254 \mathrm{~nm}$ ), $\mathrm{t}_{\mathrm{R}}($ minor enantiomer $)=14.289 \mathrm{~min}, \mathrm{t}_{\mathrm{R}}($ major enantiomer $)=21.853 \mathrm{~min}, 97 \%$ ee.

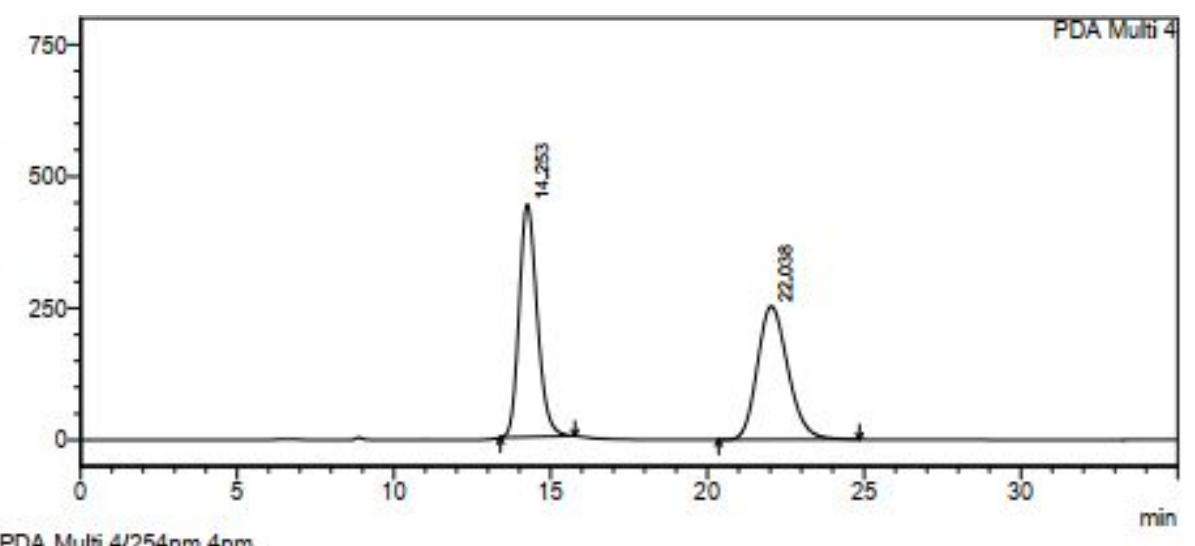

PDA Multi $4 / 254 \mathrm{~nm} 4 \mathrm{~nm}$

PeskTable
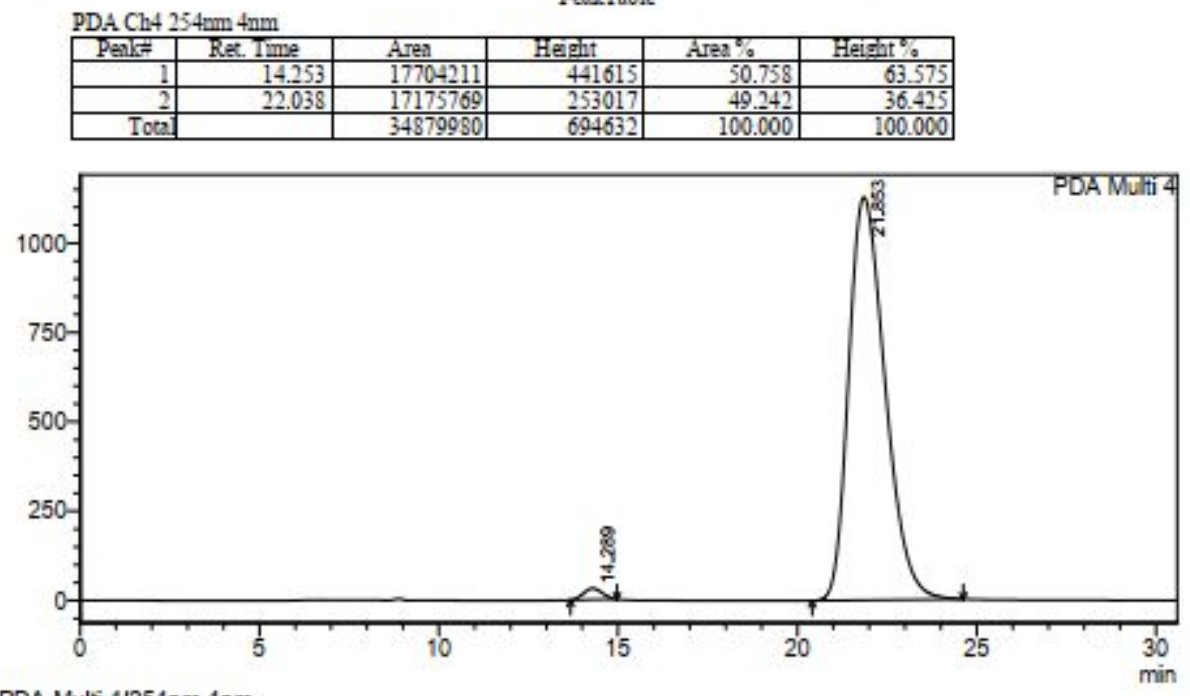

PDA Multi 4/254nm 4nm

Peni:Table

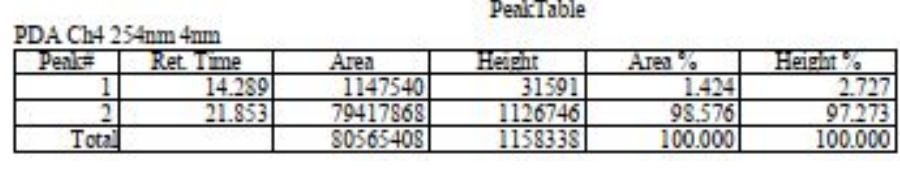

(S)-2-(3-Benzamido-1-benzyl-6-bromo-2-oxoindolin-3-yl)ethane-1-sulfonyl fluoride (3k) 
<smiles>O=C(N[C@]1(CCS(=O)(=O)F)C(=O)N(Cc2ccccc2)c2cc(Br)ccc21)Pc1ccccc1</smiles>

3k

Petroleum ether/ethyl acetate $=8: 1$ was used as the eluent for column chromatography. White solid (49 mg, $93 \%$ ), m. p. $188.7-189^{\circ} \mathrm{C} .{ }^{1} \mathbf{H}$ NMR $\left(400 \mathrm{MHz}, \mathrm{CDCl}_{3}\right) \delta 7.77(\mathrm{~d}, J=7.2 \mathrm{~Hz}, 2 \mathrm{H}), 7.57-7.49$ (m, 1H), 7.46-7.28 (m, 7H), 7.25-7.14 (m, 2H), $6.94(\mathrm{~d}, J=1.2 \mathrm{~Hz}, 1 \mathrm{H}), 5.00(\mathrm{~d}, J=16.0 \mathrm{~Hz}, 1 \mathrm{H}), 4.93(\mathrm{~d}$, $J=16.0 \mathrm{~Hz}, 1 \mathrm{H}), 3.83-3.69(\mathrm{~m}, 1 \mathrm{H}), 3.53-3.40(\mathrm{~m}, 1 \mathrm{H}), 2.80$ (ddd, $J=14.8,9.6,5.6 \mathrm{~Hz}, 1 \mathrm{H}), 2.51-2.35$ (m, 1H). ${ }^{13} \mathbf{C}$ NMR $\left(101 \mathrm{MHz}, \mathrm{CDCl}_{3}\right) \delta 174.86,166.75,143.56,134.74,132.51,132.08,129.17,128.76$, 128.16, 127.34, 127.27, 126.49, 124.29, 123.57, 113.38, 59.49, 44.74 (d, $\left.J_{\mathrm{C}-\mathrm{F}}=18.3 \mathrm{~Hz}\right), 44.54,30.13$. ${ }^{19} \mathbf{F}$ NMR $\left(376 \mathrm{MHz}, \mathrm{CDCl}_{3}\right) \delta$ 53.39. HRMS (ESI) $\mathrm{m} / \mathrm{z}$ : Calcd. for $\mathrm{C}_{24} \mathrm{H}_{20} \mathrm{~N}_{2} \mathrm{O}_{4} \mathrm{FSBrNa}$, $[\mathrm{M}+\mathrm{Na}]^{+}$, 553.0203; found: 553.0214. $[\alpha]_{\mathrm{D}}^{20}=+26.0\left(\mathrm{c} 1.0, \mathrm{CH}_{2} \mathrm{Cl}_{2}\right)$. Chiral HPLC analysis (Chiral pak AS-H, $i$ $\mathrm{PrOH} / n$-hexane $=20: 80$, flow rate $=0.5 \mathrm{~mL} / \mathrm{min}$, wave length $=254 \mathrm{~nm}), \mathrm{t}_{\mathrm{R}}($ minor enantiomer $)=$ $14.069 \mathrm{~min}, \mathrm{t}_{\mathrm{R}}$ (major enantiomer) $=21.708 \mathrm{~min}, 99 \%$ ee.

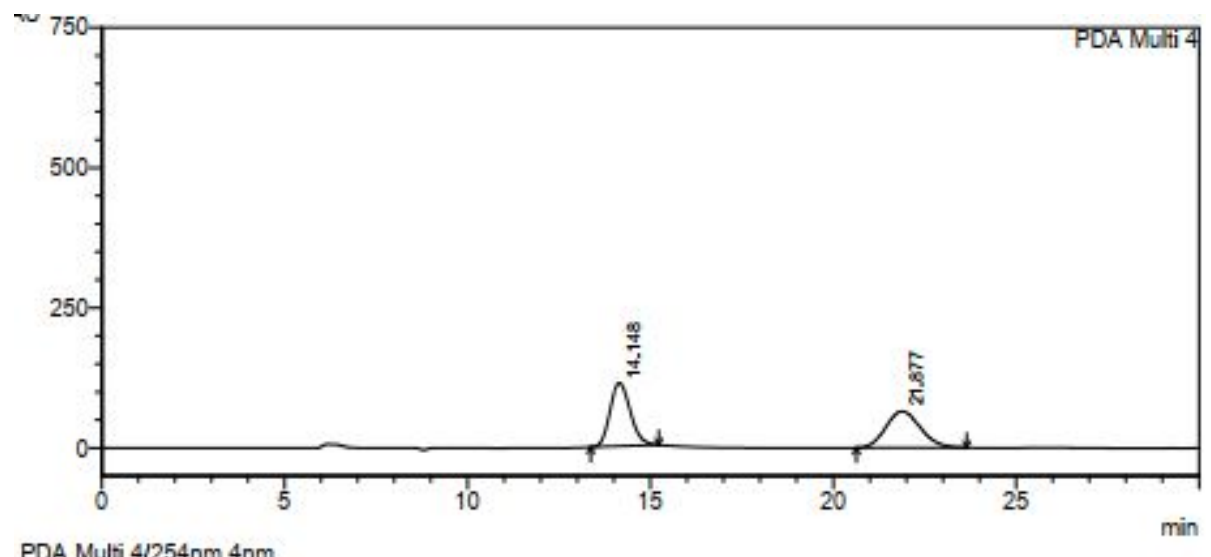

PDA Multi 4/254nm 4nm

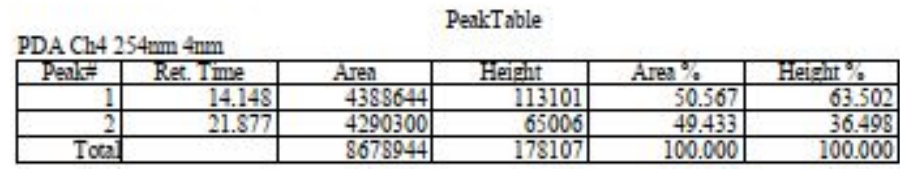




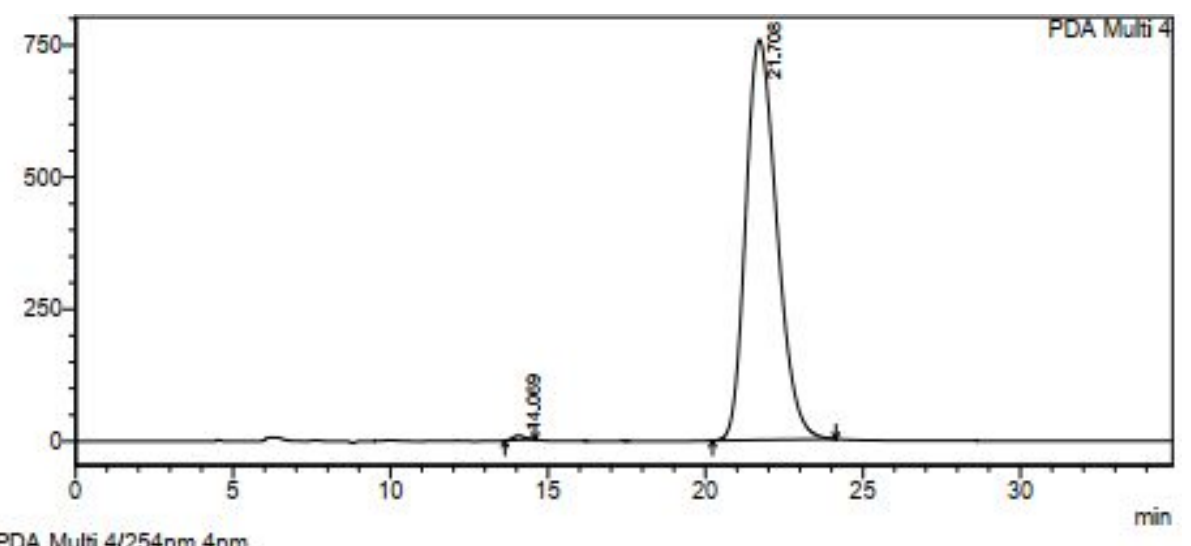

PDA Multi $4 / 254 \mathrm{~nm} 4 \mathrm{~nm}$

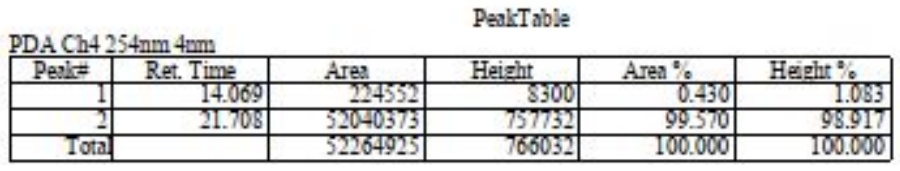

(S)-2-(3-Benzamido-1-benzyl-6-methoxy-2-oxoindolin-3-yl)ethane-1-sulfonyl fluoride (3I)<smiles>COc1ccc2c(c1)N(Cc1ccccc1)C(=O)[C@]2(CCS(=O)(=O)F)NC(=O)c1ccccc1</smiles>

3I

Petroleum ether/ethyl acetate $=8: 1$ was used as the eluent for column chromatography. White solid (47.5 mg, 99\%), m. p. 189.4-190.4 ${ }^{\circ} \mathrm{C} .{ }^{1} \mathbf{H}$ NMR (400 MHz, $\left.\mathrm{CDCl}_{3}\right) \delta$ 7.81-7.75 (m, 2H), 7.54-7.48 (m, 1H), 7.45-7.37 (m, 7H), 7.35-7.29 (m, 2H), $6.55(\mathrm{dd}, J=8.4,2.4 \mathrm{~Hz}, 1 \mathrm{H}), 6.41$ (d, $J=2.0 \mathrm{~Hz}, 1 \mathrm{H}), 5.02$ $(\mathrm{d}, J=15.6 \mathrm{~Hz}, 1 \mathrm{H}), 4.93(\mathrm{~d}, J=15.6 \mathrm{~Hz}, 1 \mathrm{H}), 3.74(\mathrm{~s}, 3 \mathrm{H}), 3.64-3.54(\mathrm{~m}, 1 \mathrm{H}), 3.49-3.40$ (m, 1H), 2.86$2.77(\mathrm{~m}, 1 \mathrm{H}), 2.57-2.48(\mathrm{~m}, 1 \mathrm{H}) .{ }^{13} \mathbf{C}$ NMR $\left(101 \mathrm{MHz}, \mathrm{CDCl}_{3}\right) \delta 175.35,166.70,161.17,143.61,135.25$, 132.60, 132.28, 129.03, 128.68, 127.96, 127.35, 127.28, 124.38, 120.04, 107.20, 98.11, 59.62, 55.48, $45.08\left(\mathrm{~d}, J_{\mathrm{C}-\mathrm{F}}=18.1 \mathrm{~Hz}\right), 44.46,30.50 .{ }^{19} \mathbf{F}$ NMR $\left(376 \mathrm{MHz}, \mathrm{CDCl}_{3}\right) \delta 53.23$. HRMS (ESI) $\mathrm{m} / z$ : Calcd. for $\mathrm{C}_{25} \mathrm{H}_{23} \mathrm{~N}_{2} \mathrm{O}_{5} \mathrm{FSNa}$, [M+Na] $]^{+}$, 505.1204; found: 505.1229. $[\alpha]_{\mathrm{D}}{ }^{20}=+12.4$ (c 1.0, $\mathrm{CH}_{2} \mathrm{Cl}_{2}$ ). Chiral HPLC analysis (Chiral pak AS-H, $i-\mathrm{PrOH} / n$-hexane $=20: 80$, flow rate $=0.5 \mathrm{~mL} / \mathrm{min}$, wave length $=230 \mathrm{~nm}$ ), $t_{R}($ minor enantiomer $)=20.979 \mathrm{~min}, t_{R}($ major enantiomer $)=39.970 \mathrm{~min}, 98 \%$ ee . 


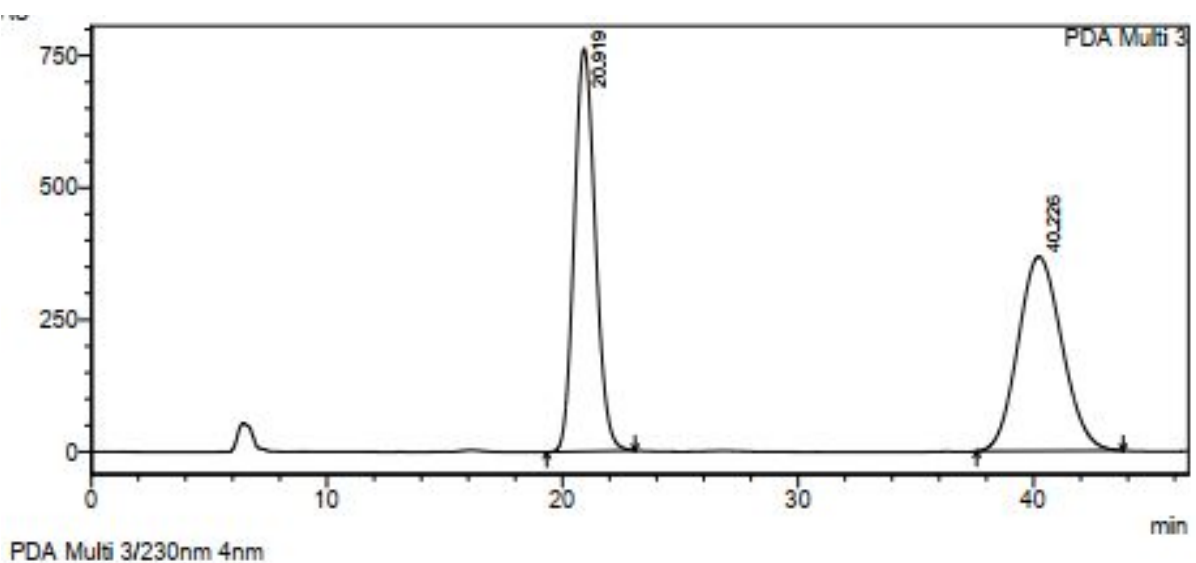

PDA Multi $3 / 230 \mathrm{~nm} 4 \mathrm{~nm}$

PeakTable
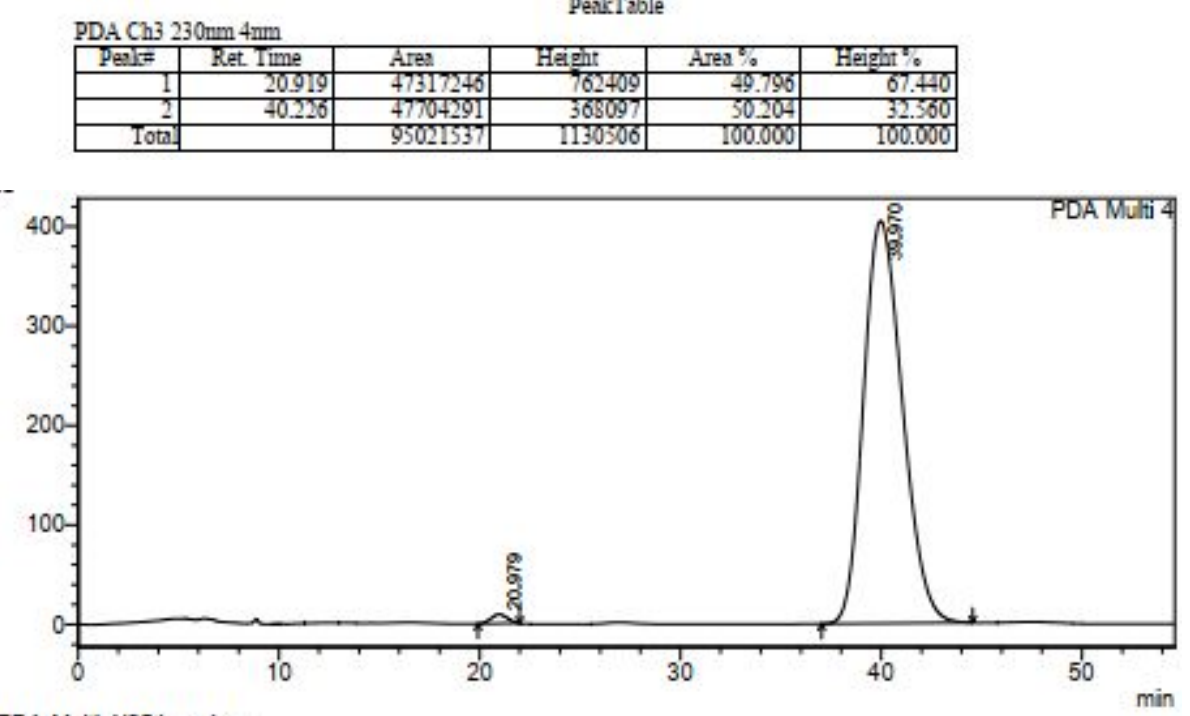

PDA Multi $4 / 254 \mathrm{~nm} 4 \mathrm{~nm}$

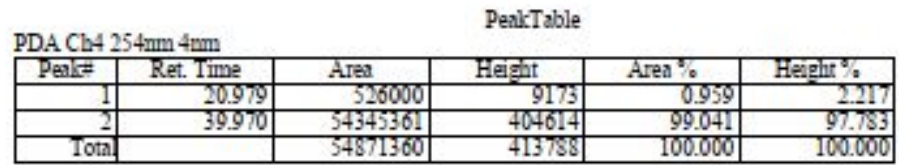

(S)-2-(3-Benzamido-1-benzyl-7-fluoro-2-oxoindolin-3-yl)ethane-1-sulfonyl fluoride (3m)<smiles>O=C1N(Cc2ccccc2)c2c(F)cccc2[C@]1(CCS(=O)(=O)F)NC(=O)P(=O)(F)F</smiles>

Petroleum ether/ethyl acetate $=8: 1$ was used as the eluent for column chromatography. White solid (46.8 mg, 99\%), m. p. $187.4-188.7{ }^{\circ} \mathrm{C} .{ }^{1} \mathbf{H}$ NMR (400 MHz, $\left.\mathrm{CDCl}_{3}\right) \delta$ 7.82-7.71 (m, 2H), 7.54-7.48 (m, 1H), 7.46-7.32 (m, 6H), 7.33-7.26 (m, 2H), 7.18-7.09 (m, 1H), 7.07-6.97 (m, 2H), 5.17 (d, $J=15.6 \mathrm{~Hz}$, $1 \mathrm{H}), 5.08(\mathrm{~d}, J=15.6 \mathrm{~Hz}, 1 \mathrm{H}), 3.68-3.57(\mathrm{~m}, 1 \mathrm{H}), 3.42-3.32(\mathrm{~m}, 1 \mathrm{H}), 2.82-2.71(\mathrm{~m}, 1 \mathrm{H}), 2.49-2.38(\mathrm{~m}$, 1H) ${ }^{13} \mathbf{C}$ NMR $\left(101 \mathrm{MHz}, \mathrm{CDCl}_{3}\right) \delta 174.81,166.69,147.73\left(\mathrm{~d}, J_{\mathrm{C}-\mathrm{F}}=245.9 \mathrm{~Hz}\right), 136.58,132.45,132.08$, $131.37\left(\mathrm{~d}, J_{\mathrm{C}-\mathrm{F}}=2.8 \mathrm{~Hz}\right), 128.83,128.72,127.92,127.53,127.35,124.42\left(\mathrm{~d}, J_{\mathrm{C}-\mathrm{F}}=6.5 \mathrm{~Hz}\right), 118.70(\mathrm{~d}$, 
$\left.J_{\mathrm{C}-\mathrm{F}}=3.1 \mathrm{~Hz}\right), 118.15\left(\mathrm{~d}, J_{\mathrm{C}-\mathrm{F}}=19.7 \mathrm{~Hz}\right), 59.94,46.07\left(\mathrm{~d}, J_{\mathrm{C}-\mathrm{F}}=4.7 \mathrm{~Hz}\right), 44.68\left(\mathrm{~d}, J_{\mathrm{C}-\mathrm{F}}=18.4 \mathrm{~Hz}\right), 30.50$. ${ }^{19} \mathbf{F}$ NMR $\left(376 \mathrm{MHz}, \mathrm{CDCl}_{3}\right) \delta$-132.29. ${ }^{19} \mathbf{F}$ NMR $\left(376 \mathrm{MHz}, \mathrm{CDCl}_{3}\right) \delta 53.34$. HRMS (ESI) $\mathrm{m} / z$ : Calcd. for $\mathrm{C}_{24} \mathrm{H}_{20} \mathrm{~N}_{2} \mathrm{O}_{4} \mathrm{~F}_{2} \mathrm{SNa}$, $[\mathrm{M}+\mathrm{Na}]^{+}$, 493.1004; found: $493.1022[\alpha]_{\mathrm{D}}{ }^{20}=+18.7\left(\mathrm{c} 1.0, \mathrm{CH}_{2} \mathrm{Cl}_{2}\right)$. Chiral HPLC analysis (Chiral pak AS-H, $i-\mathrm{PrOH} / n$-hexane $=20: 80$, flow rate $=0.5 \mathrm{~mL} / \mathrm{min}$, wave length $=$ $254 \mathrm{~nm}), \mathrm{t}_{\mathrm{R}}($ minor enantiomer $)=14.500 \mathrm{~min}, \mathrm{t}_{\mathrm{R}}($ major enantiomer $)=34.242 \mathrm{~min}, 98 \%$ ee.

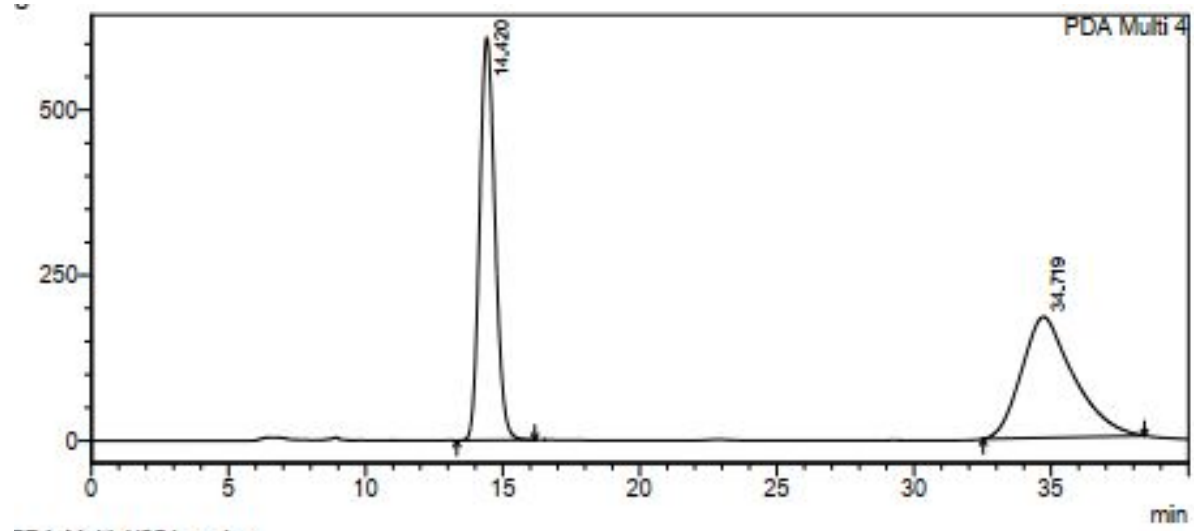

गDA Multi 4/254nm 4nm
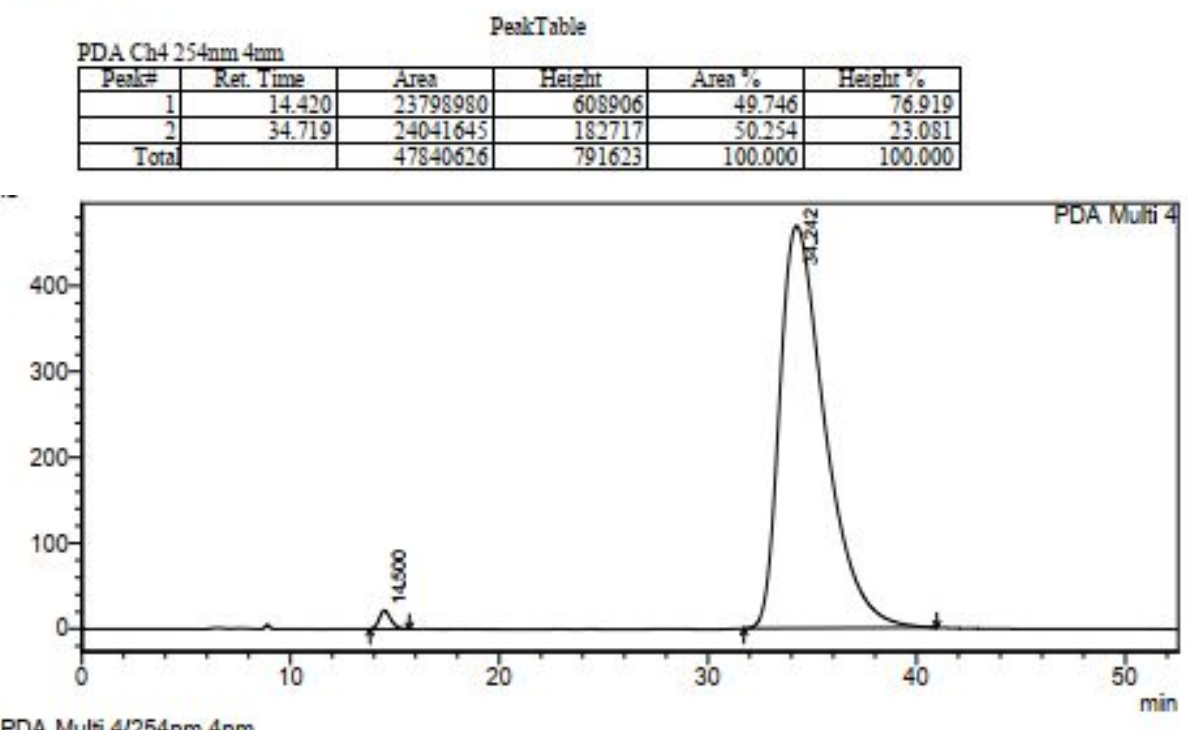

1 PDA Multi 4/254nm 4nm

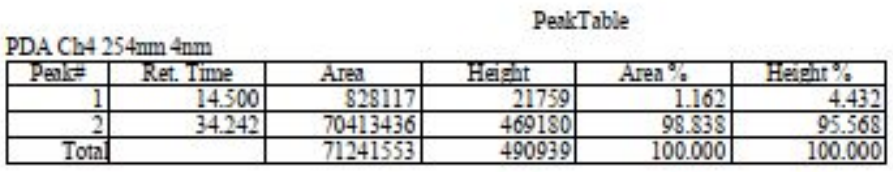

(S)-2-(3-Benzamido-1-benzyl-7-chloro-2-oxoindolin-3-yl)ethane-1-sulfonyl fluoride (3n)<smiles>O=C(N[C@]1(CCS(=O)(=O)F)C(=O)N(Cc2ccccc2)c2c(Cl)cccc21)Pc1ccccc1</smiles>

$3 n$

Petroleum ether/ethyl acetate $=8: 1$ was used as the eluent for column chromatography. White solid (45 mg, $93 \%)$, m. p. $177.9-178.5^{\circ} \mathrm{C} .{ }^{1} \mathbf{H}$ NMR $\left(400 \mathrm{MHz}, \mathrm{CDCl}_{3}\right) \delta 7.74(\mathrm{~d}, J=7.2 \mathrm{~Hz}, 2 \mathrm{H}), 7.55-7.47(\mathrm{~m}$, 
1H), 7.43-7.31 (m, 7H), 7.30-7.26 (m, 1H), 7.26-7.22 (m, 2H), 7.04-6.97 (m, 1H), 5.50 (d, J=16.0 Hz, $1 \mathrm{H}), 5.34(\mathrm{~d}, J=16.4 \mathrm{~Hz}, 1 \mathrm{H}), 3.78-3.64(\mathrm{~m}, 1 \mathrm{H}), 3.51-3.37(\mathrm{~m}, 1 \mathrm{H}), 2.77$ (ddd, $J=14.8,9.6,5.2 \mathrm{~Hz}$, $1 \mathrm{H}), 2.49-2.37(\mathrm{~m}, 1 \mathrm{H}) .{ }^{13} \mathbf{C}$ NMR $\left(101 \mathrm{MHz}, \mathrm{CDCl}_{3}\right) \delta 175.79,166.62,138.36,137.17,132.45,131.99$, $131.55,128.79,128.72$, 127.46, 127.36, 126.65, 124.48, 121.23, 116.38, 59.35, 44.71 (d, $\left.J_{\mathrm{C}-\mathrm{F}}=18.2 \mathrm{~Hz}\right)$, 44.62, 30.67. ${ }^{19}$ F NMR $\left(376 \mathrm{MHz}, \mathrm{CDCl}_{3}\right) \delta$ 53.35. HRMS (ESI) $\mathrm{m} / \mathrm{z}$ : Calcd. for $\mathrm{C}_{24} \mathrm{H}_{20} \mathrm{~N}_{2} \mathrm{O}_{4} \mathrm{FSCINa}$, $[\mathrm{M}+\mathrm{Na}]^{+}, 509.0709$; found: 509.0729. $[\alpha]_{\mathrm{D}}^{20}=+46.2\left(\mathrm{c} 1.0, \mathrm{CH}_{2} \mathrm{Cl}_{2}\right)$. Chiral HPLC analysis (Chiral pak AS-H, $i-\mathrm{PrOH} / n$-hexane $=20: 80$, flow rate $=0.5 \mathrm{~mL} / \mathrm{min}$, wave length $=254 \mathrm{~nm}), \mathrm{t}_{\mathrm{R}}$ (minor enantiomer) $=14.718 \mathrm{~min}, \mathrm{t}_{\mathrm{R}}$ (major enantiomer $)=34.453 \mathrm{~min}, 99 \%$ ee.

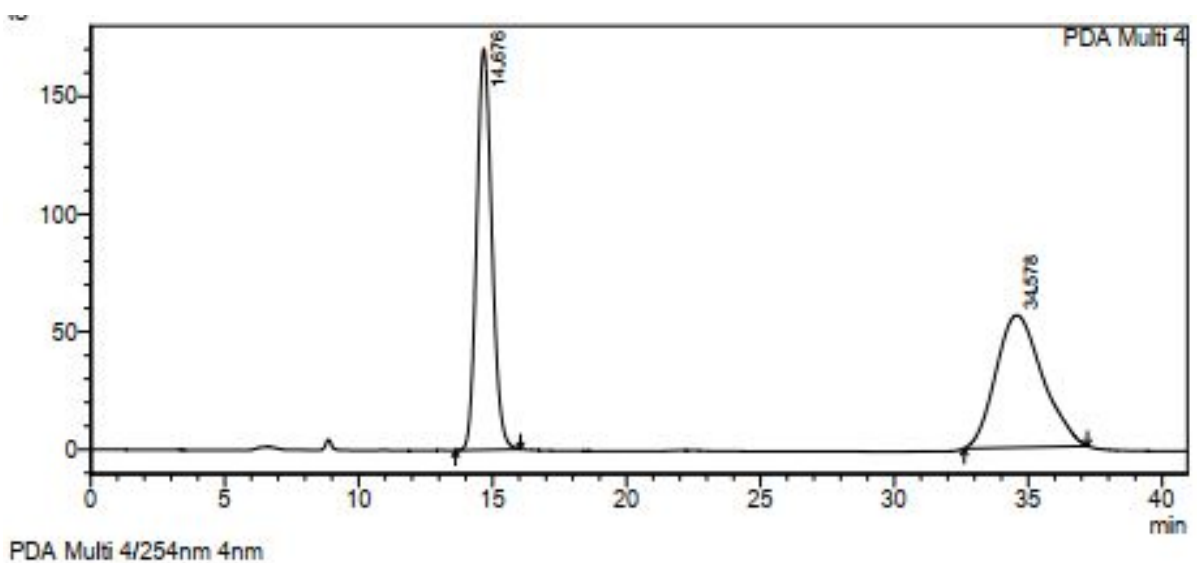

PDA Multi 4/254nm 4nm
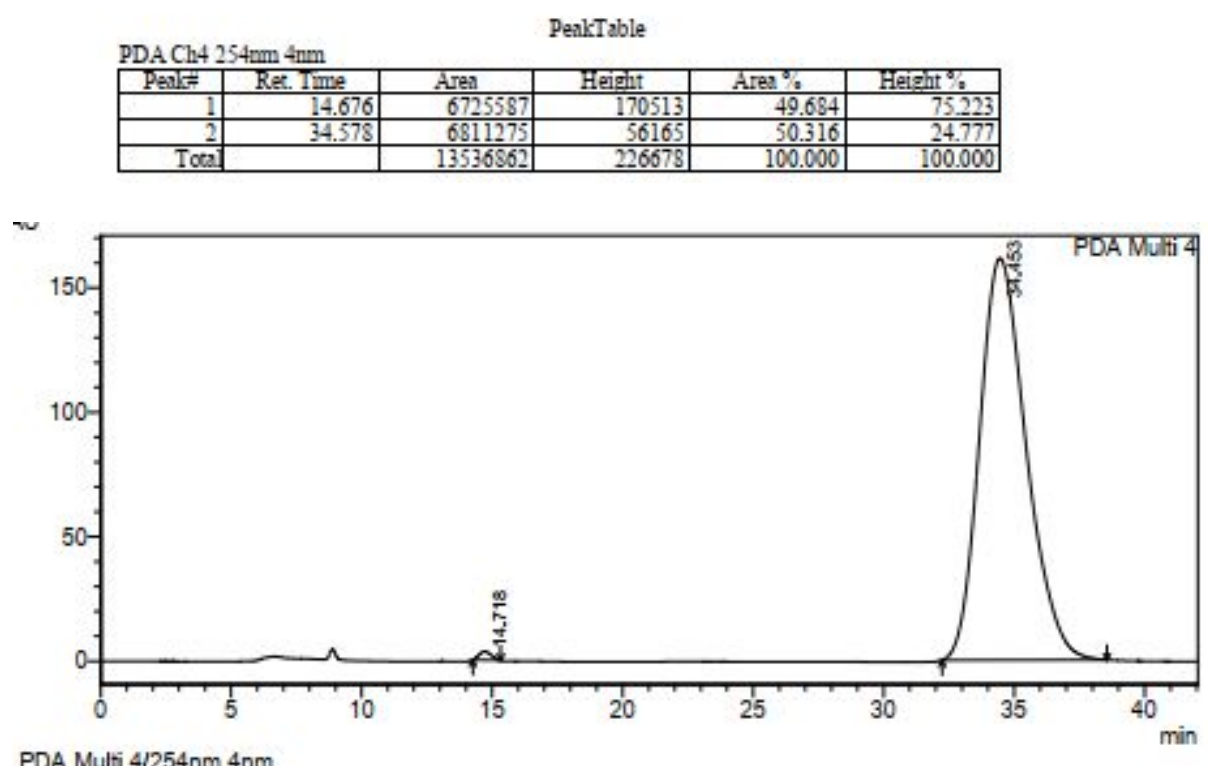

PDA Multi 4/254nm 4nm

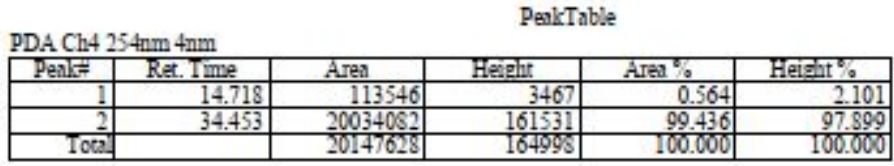

(S)-2-(3-Benzamido-1-benzyl-7-bromo-2-oxoindolin-3-yl)ethane-1-sulfonyl fluoride (3o) 
<smiles>O=C(N[C@]1(CCS(=O)(=O)F)C(=O)N(Cc2ccccc2)c2c(Br)cccc21)c1ccccc1</smiles>

30

Petroleum ether/ethyl acetate $=8: 1$ was used as the eluent for column chromatography. White solid (49.1 mg, 93\%), m. p. $182.5-183.8^{\circ} \mathrm{C} .{ }^{1} \mathbf{H}$ NMR (400 MHz, $\left.\mathrm{CDCl}_{3}\right) \delta$ 7.78-7.74 (m, 2H), 7.55-7.49 (m, 1H), 7.46-7.34 (m, 7H), 7.33-7.26 (m, 3H), 6.97-6.92 (m, 1H), 5.57 (d, $J=16.4 \mathrm{~Hz}, 1 \mathrm{H}), 5.38$ (d, $J=$ $16.4 \mathrm{~Hz}, 1 \mathrm{H}), 3.80-3.70(\mathrm{~m}, 1 \mathrm{H}), 3.49-3.39(\mathrm{~m}, 1 \mathrm{H}), 2.79$ (ddd, $J=14.4,9.2,5.2 \mathrm{~Hz}, 1 \mathrm{H}), 2.42$ (ddd, $J$ $=14.8,9.2,6.0 \mathrm{~Hz}, 1 \mathrm{H}) .{ }^{13} \mathbf{C}$ NMR $\left(101 \mathrm{MHz}, \mathrm{CDCl}_{3}\right) \delta 175.84,166.56,139.81,137.12,135.81,132.46$, $132.02,131.91,128.74,127.34,127.31,126.53,124.77,121.82,103.37,59.14,45.19,44.65\left(\mathrm{~d}, J_{\mathrm{C}-\mathrm{F}}=\right.$ $18.2 \mathrm{~Hz}$ ), 30.69. ${ }^{19} \mathbf{F}$ NMR $\left(376 \mathrm{MHz}, \mathrm{CDCl}_{3}\right) \delta 53.31$. HRMS (ESI) $\mathrm{m} / \mathrm{z}$ : Calcd. for $\mathrm{C}_{24} \mathrm{H}_{20} \mathrm{~N}_{2} \mathrm{O}_{4} \mathrm{FSBrNa}$, $[\mathrm{M}+\mathrm{Na}]^{+}, 553.0203$; found: 553.0216. $[\alpha]_{\mathrm{D}}{ }^{20}=+50.8\left(\mathrm{c} 1.0, \mathrm{CH}_{2} \mathrm{Cl}_{2}\right)$. Chiral HPLC analysis (Chiral pak AS-H, $i-\mathrm{PrOH} / n$-hexane $=20: 80$, flow rate $=0.5 \mathrm{~mL} / \mathrm{min}$, wave length $=220 \mathrm{~nm}), \mathrm{t}_{\mathrm{R}}($ minor enantiomer) $=19.223 \mathrm{~min}, \mathrm{t}_{\mathrm{R}}$ (major enantiomer) $=50.930 \mathrm{~min}, 98 \%$ ee.

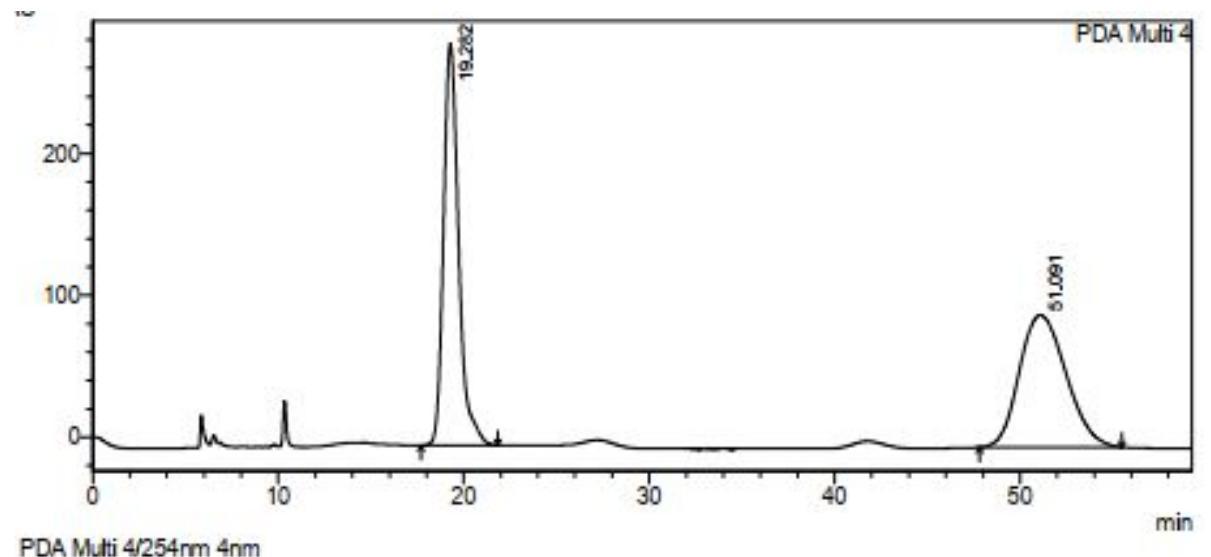

PDA Muti 4/254rm 4nm

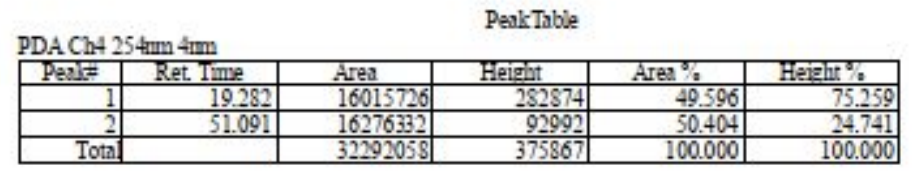




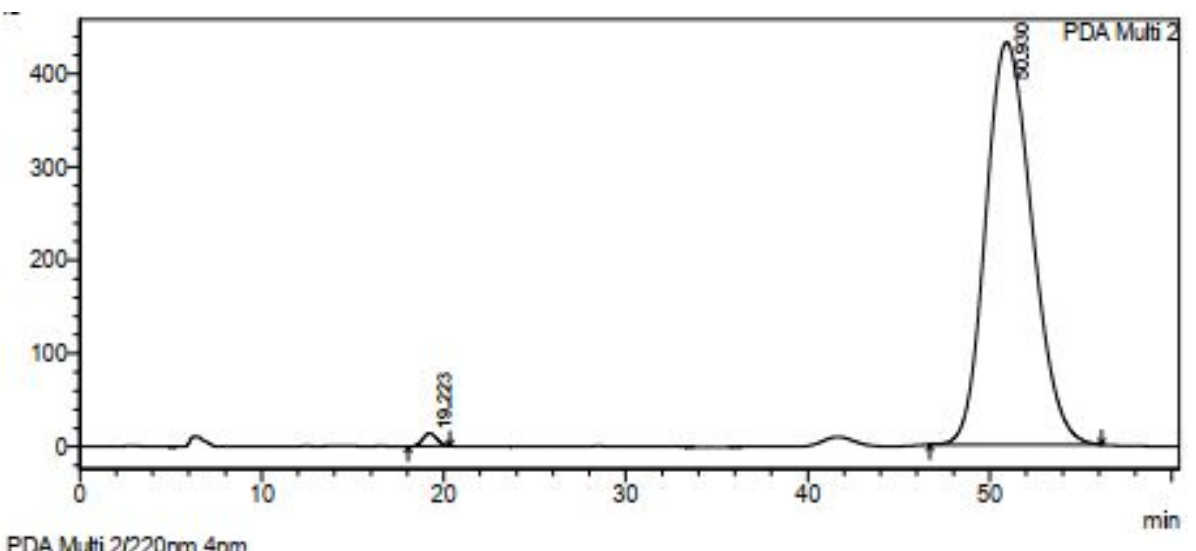

PDA Mutti $2 / 220 \mathrm{~mm} 4 \mathrm{~nm}$

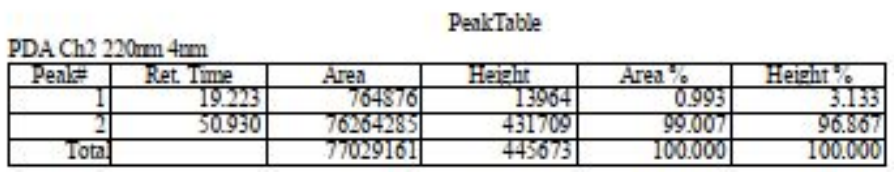

(S)-2-(3-Benzamido-1-benzyl-2-oxo-7-(trifluoromethyl)indolin-3-yl)ethane-1-sulfonyl fluoride (3p)<smiles>O=C(N[C@]1(CCS(=O)(=O)F)C(=O)N(Cc2ccccc2)c2c(C(F)(F)F)cccc21)c1ccccc1</smiles>

$3 p$

Petroleum ether/ethyl acetate $=8: 1$ was used as the eluent for column chromatography. White solid (41 mg, 86\%), m. p. $211.6-212.3^{\circ} \mathrm{C} .{ }^{1} \mathbf{H}$ NMR $\left(400 \mathrm{MHz}, \mathrm{CDCl}_{3}\right) \delta$ 7.76-7.67 (m, $\left.2 \mathrm{H}\right), 7.62(\mathrm{~d}, J=8.0 \mathrm{~Hz}$, $1 \mathrm{H}), 7.53-7.44(\mathrm{~m}, 3 \mathrm{H}), 7.42-7.21(\mathrm{~m}, 7 \mathrm{H}), 7.18-7.11(\mathrm{~m}, 1 \mathrm{H}), 5.27-5.16(\mathrm{~m}, 2 \mathrm{H}), 3.75-3.62(\mathrm{~m}, 1 \mathrm{H})$, 3.50-3.39 (m, 1H), 2.81-2.69 (m, 1H), 2.46-2.33 (m, 1H). ${ }^{13} \mathbf{C}$ NMR (101 MHz, $\left.\mathrm{CDCl}_{3}\right) \delta$ 176.62, 166.77, $140.54,136.05,132.54,131.88,131.76,128.75,128.58,127.98$ (d, $\left.J_{\mathrm{C}-\mathrm{F}}=6.1 \mathrm{~Hz}\right), 127.37,127.24,126.27$, $126.01,123.15,113.63\left(\mathrm{~d}, J_{\mathrm{C}-\mathrm{F}}=32.9 \mathrm{~Hz}\right), 58.11,46.56\left(\mathrm{~d}, J_{\mathrm{C}-\mathrm{F}}=4.5 \mathrm{~Hz}\right), 44.59\left(\mathrm{~d}, J_{\mathrm{C}-\mathrm{F}}=18.1 \mathrm{~Hz}\right)$, 30.51. ${ }^{19} \mathbf{F}$ NMR $\left(376 \mathrm{MHz}, \mathrm{CDCl}_{3}\right) \delta 53.47,-54.62$. HRMS (ESI) $m / z$ : Calcd. for $\mathrm{C}_{25} \mathrm{H}_{20} \mathrm{~N}_{2} \mathrm{O}_{4} \mathrm{~F}_{4} \mathrm{SNa}$, $[\mathrm{M}+\mathrm{Na}]^{+}, 543.0972$; found: 543.0988. $[\boldsymbol{\alpha}]_{\mathrm{D}}^{20}=+45.9\left(\mathrm{c} 1.0, \mathrm{CH}_{2} \mathrm{Cl}_{2}\right)$. Chiral HPLC analysis (Chiral pak AS- $\mathrm{H}, i-\mathrm{PrOH} / n$-hexane $=20: 80$, flow rate $=0.5 \mathrm{~mL} / \mathrm{min}$, wave length $=254 \mathrm{~nm}), \mathrm{t}_{\mathrm{R}}($ minor enantiomer $)$ $=10.377 \mathrm{~min}, \mathrm{t}_{\mathrm{R}}($ major enantiomer $)=22.496 \mathrm{~min}, 98 \%$ ee. 


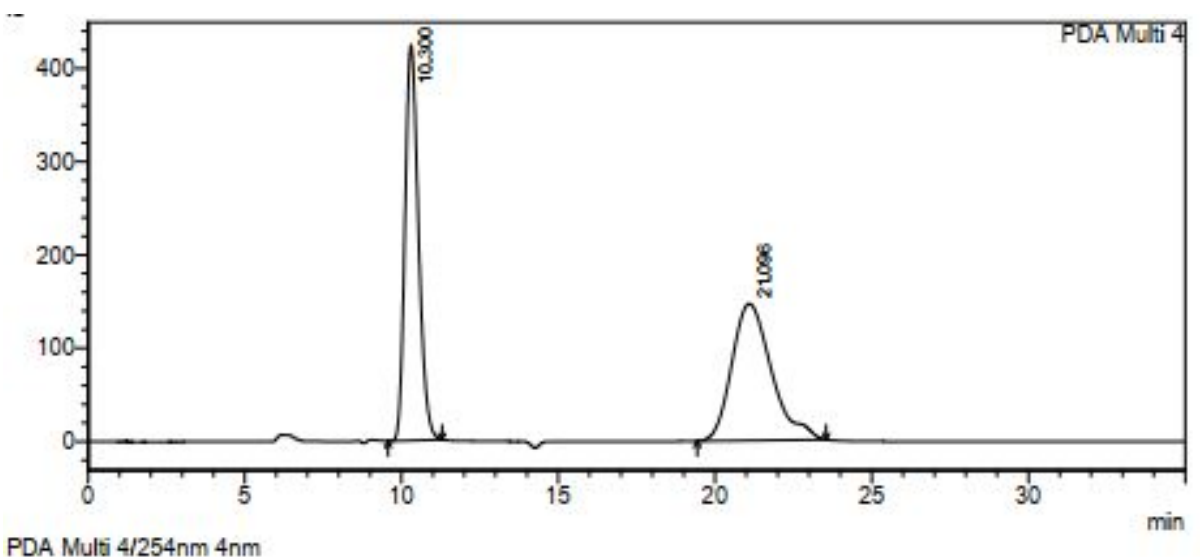

PDA Multi 4/254nm 4nm
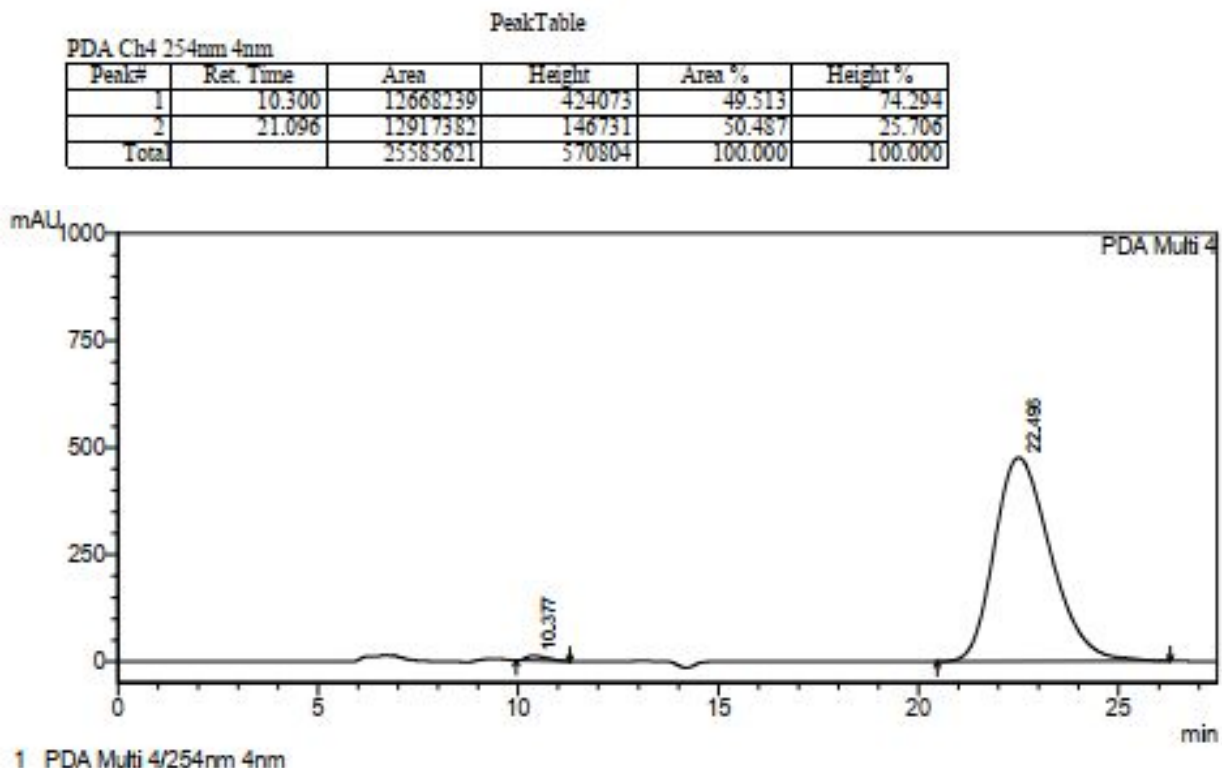

1 PDA Muti 4/254rm 4nm

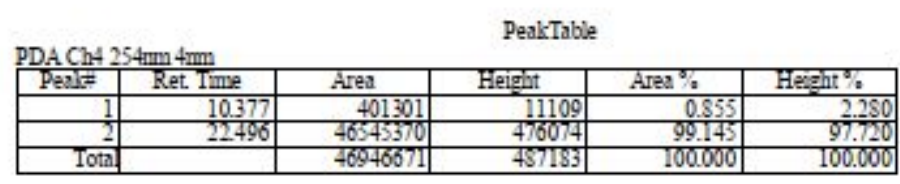

(S)-2-(3-Benzamido-1-benzyl-5,7-dimethyl-2-oxoindolin-3-yl)ethane-1-sulfonyl fluoride (3q)<smiles>Cc1cc(C)c2c(c1)[C@](CCS(=O)(=O)F)(C(=O)c1ccccc1)C(=O)N2Cc1ccccc1</smiles>

$3 q$

Petroleum ether/ethyl acetate $=8: 1$ was used as the eluent for column chromatography. White solid (36.6 mg, 83\%), m. p. $220.4-221.7^{\circ} \mathrm{C} .{ }^{1} \mathbf{H}$ NMR $\left(500 \mathrm{MHz}, \mathrm{CDCl}_{3}\right) \delta 7.79(\mathrm{~d}, J=7.5 \mathrm{~Hz}, 2 \mathrm{H}), 7.54-$ $7.48(\mathrm{~m}, 1 \mathrm{H}), 7.43-7.26(\mathrm{~m}, 8 \mathrm{H}), 7.03(\mathrm{~s}, 1 \mathrm{H}), 6.82(\mathrm{~s}, 1 \mathrm{H}), 5.30(\mathrm{~d}, J=17.0 \mathrm{~Hz}, 1 \mathrm{H}), 5.16(\mathrm{~d}, J=17.0$ $\mathrm{Hz}, 1 \mathrm{H}), 3.81-3.72(\mathrm{~m}, 1 \mathrm{H}), 3.54-3.46(\mathrm{~m}, 1 \mathrm{H}), 2.84-2.76(\mathrm{~m}, 1 \mathrm{H}), 2.49-2.41(\mathrm{~m}, 1 \mathrm{H}), 2.24(\mathrm{~s}, 6 \mathrm{H}) .{ }^{13} \mathrm{C}$ NMR $\left(126 \mathrm{MHz}, \mathrm{CDCl}_{3}\right) \delta 175.92,166.52,137.78,137.35,134.35,133.30,132.47,132.23,129.35$, $129.02,128.62,127.38,127.34,125.85,121.37,120.36,59.37,45.64,45.01\left(\mathrm{~d}, J_{\mathrm{C}-\mathrm{F}}=17.9 \mathrm{~Hz}\right), 30.93$, 
20.80, 18.64. ${ }^{19} \mathbf{F}$ NMR $\left(471 \mathrm{MHz}, \mathrm{CDCl}_{3}\right) \delta$ 53.19. HRMS (ESI) $\mathrm{m} / z$ : Calcd. for $\mathrm{C}_{26} \mathrm{H}_{25} \mathrm{~N}_{2} \mathrm{O}_{4} \mathrm{FSNa}$, $[\mathrm{M}+\mathrm{Na}]^{+}, 503.1411$, found: 503.1428. $[\alpha]_{\mathrm{D}}{ }^{20}=-12.3\left(\mathrm{c} 1.0, \mathrm{CH}_{2} \mathrm{Cl}_{2}\right)$. Chiral HPLC analysis (Chiral pak AS-H, $i-\mathrm{PrOH} / n$-hexane $=20: 80$, flow rate $=0.5 \mathrm{~mL} / \mathrm{min}$, wave length $=254 \mathrm{~nm}), \mathrm{t}_{\mathrm{R}}$ (minor enantiomer)

$=14.327 \mathrm{~min}, \mathrm{t}_{\mathrm{R}}$ (major enantiomer) $=32.837 \mathrm{~min}, 99 \%$ ee.

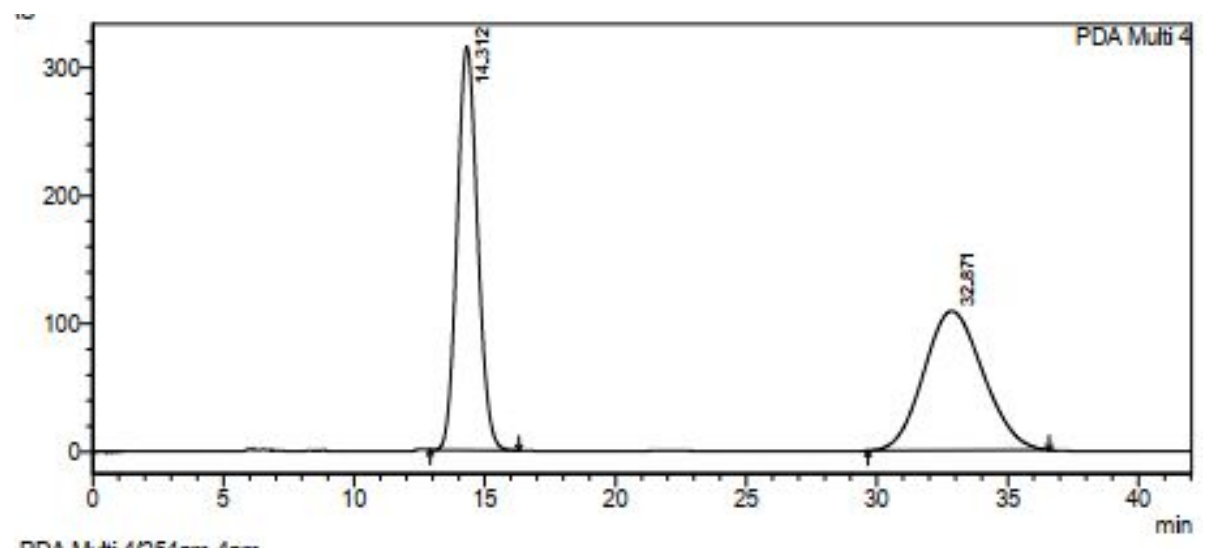

PDA Muti 4/254rm 4nm
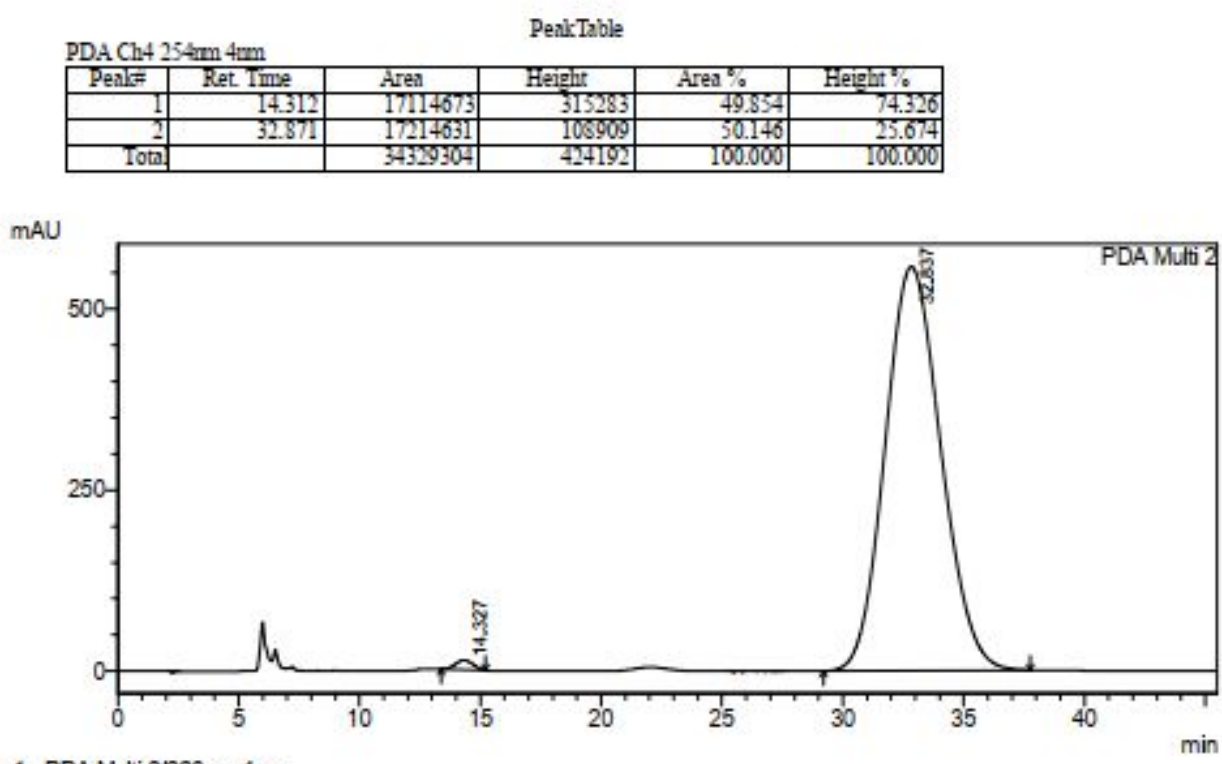

1 PDA Muti $2 / 220 \mathrm{rm} 4 \mathrm{~nm}$

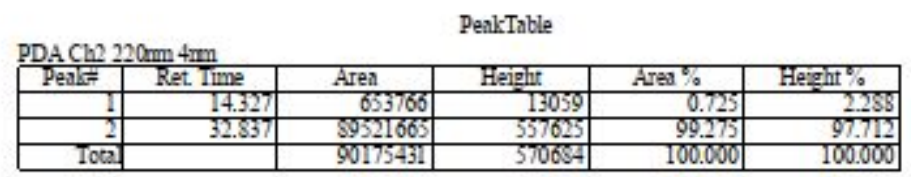

(S)-2-(3-(2-Naphthamido)-1-benzyl-2-oxoindolin-3-yl)ethane-1-sulfonyl fluoride (3r)<smiles>O=C(N[C@]1(CCS(=O)(=O)F)C(=O)N(Cc2ccccc2)c2ccccc21)c1ccc2ccccc2c1</smiles> 
Petroleum ether/ethyl acetate $=6: 1$ was used as the eluent for column chromatography. White solid (47.6 mg, 95\%), m. p. 228.9-230.4 ${ }^{\circ} \mathrm{C} .{ }^{1} \mathbf{H}$ NMR (400 MHz, $\left.\mathrm{CDCl}_{3}\right) \delta 8.23(\mathrm{~s}, 1 \mathrm{H}), 7.85-7.72(\mathrm{~m}, 4 \mathrm{H})$, 7.58-7.48 (m, 3H), 7.46-7.36 (m, 5H), 7.34-7.28 (m, 1H), 7.27-7.21 (m, 1H), 7.06 (td, $J=7.6,0.8 \mathrm{~Hz}$, $1 \mathrm{H}), 6.81(\mathrm{~d}, J=8.0 \mathrm{~Hz}, 1 \mathrm{H}), 5.06(\mathrm{~d}, J=16.0 \mathrm{~Hz}, 1 \mathrm{H}), 5.00(\mathrm{~d}, J=16.0 \mathrm{~Hz}, 1 \mathrm{H}), 3.79-3.67(\mathrm{~m}, 1 \mathrm{H})$, 3.54-3.43 (m, 1H), 2.95-2.84 (m, 1H), 2.63-2.51(m, 1H). ${ }^{13} \mathbf{C}$ NMR (101 MHz, $\left.\mathrm{CDCl}_{3}\right) \delta 175.22,166.79$, $142.25,135.31,135.00,132.43,129.84,129.45,129.17,129.05,128.51,128.39,128.32,128.06,127.94$, 127.71, 127.34, 126.86, 123.67, 123.43, 123.06, 110.07, 60.13, 45.06 (d, $\left.J_{\mathrm{C}-\mathrm{F}}=18.1 \mathrm{~Hz}\right), 44.47,30.49$. ${ }^{19}$ F NMR $\left(376 \mathrm{MHz}, \mathrm{CDCl}_{3}\right) \delta$ 53.36. HRMS (ESI) $m / z$ : Calcd. for $\mathrm{C}_{28} \mathrm{H}_{23} \mathrm{~N}_{2} \mathrm{O}_{4} \mathrm{FSNa},[\mathrm{M}+\mathrm{Na}]^{+}$, 525.1255; found: 525.1271. $[\alpha]_{\mathrm{D}}{ }^{20}=+24.8\left(\mathrm{c} 1.0, \mathrm{CH}_{2} \mathrm{Cl}_{2}\right)$. Chiral HPLC analysis (Chiral pak AS-H, $i$ $\mathrm{PrOH} / n$-hexane $=20: 80$, flow rate $=0.5 \mathrm{~mL} / \mathrm{min}$, wave length $=254 \mathrm{~nm}), \mathrm{t}_{\mathrm{R}}($ minor enantiomer $)=$ $18.755 \mathrm{~min}, \mathrm{t}_{\mathrm{R}}$ (major enantiomer) $=44.439 \mathrm{~min}, 99 \%$ ee.

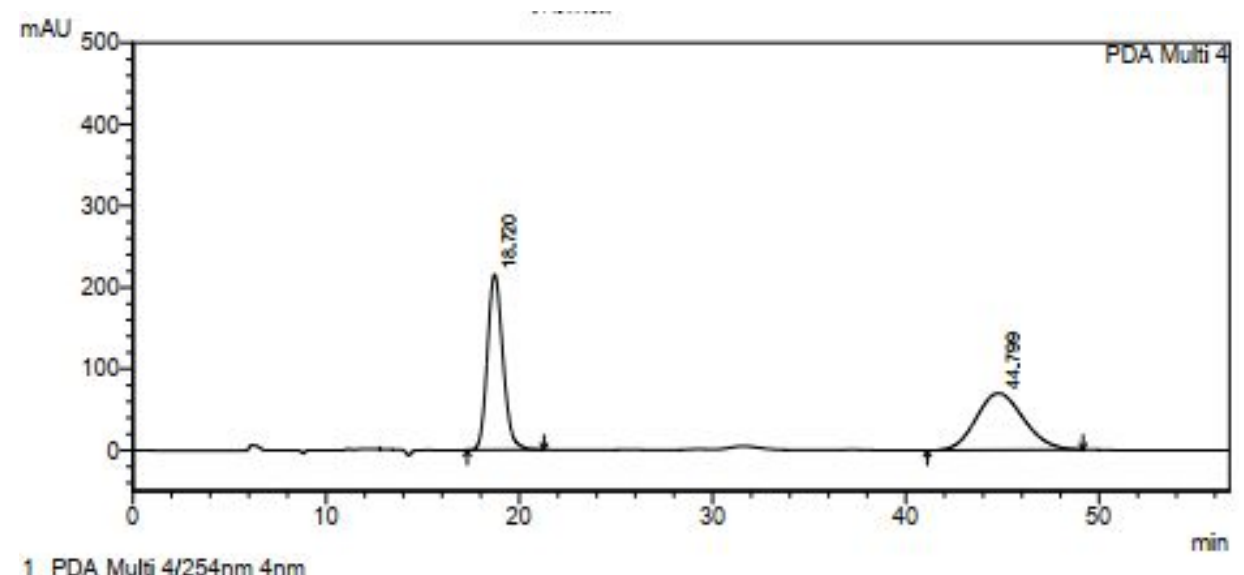

1 PDA Multi $4 / 254 \mathrm{~nm} 4 \mathrm{~nm}$
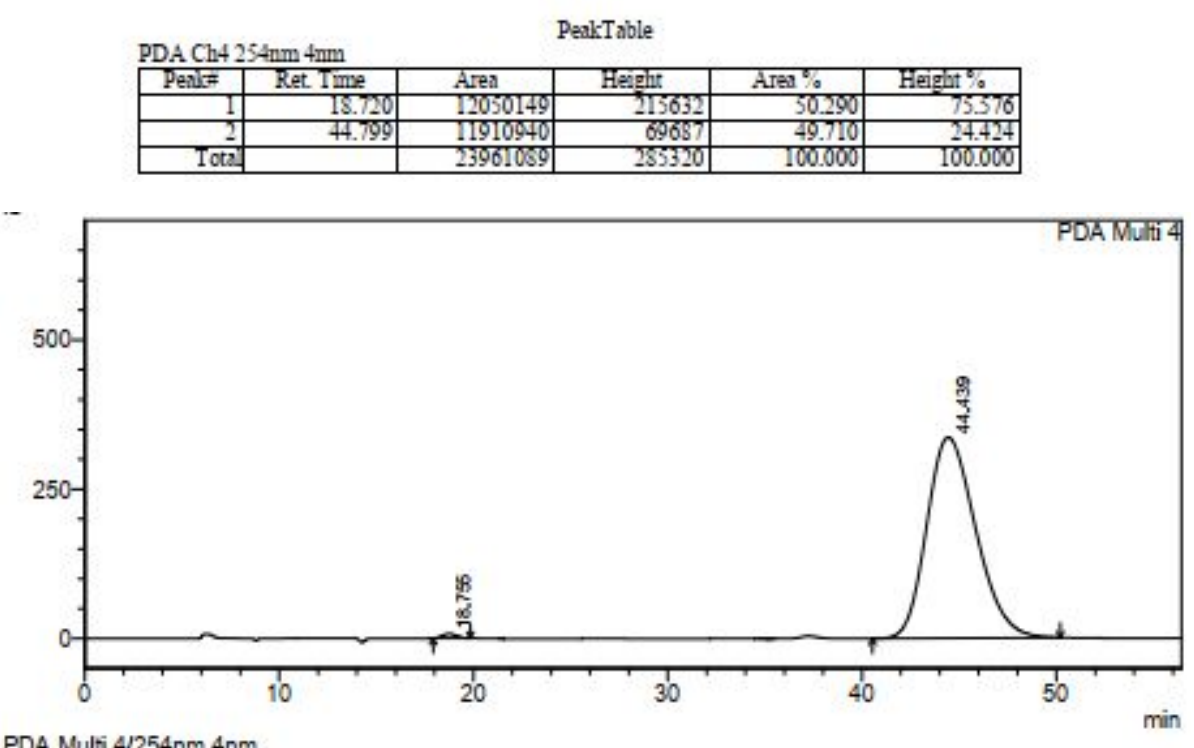

PDA Multi $4 / 254 \mathrm{~nm} 4 \mathrm{~nm}$

PevkTable

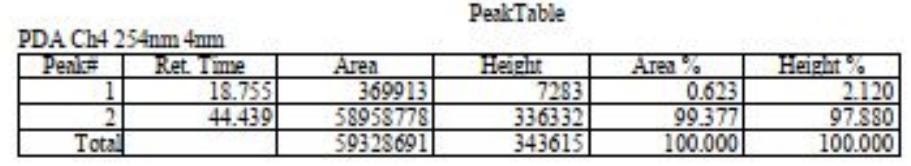

(S)-2-(1-Benzyl-3-(1H-indole-3-carboxamido)-2-oxoindolin-3-yl)ethane-1-sulfonyl fluoride (3s) 
<smiles>O=C(N[C@]1(CCS(=O)(=O)F)C(=O)N(Cc2ccccc2)c2ccccc21)c1c[nH]c2ccccc12</smiles>

Petroleum ether/ethyl acetate $=6: 1$ was used as the eluent for column chromatography. White solid (43.9 mg, 90\%), m. p. $217.9-218.6^{\circ} \mathrm{C} .{ }^{1} \mathbf{H}$ NMR (500 MHz, $\left.\mathrm{CDCl}_{3}\right) \delta 8.51(\mathrm{~d}, J=2.0 \mathrm{~Hz}, 1 \mathrm{H}), 7.99$ (d, $J=7.5 \mathrm{~Hz}, 1 \mathrm{H}), 7.59(\mathrm{~d}, J=3.0 \mathrm{~Hz}, 1 \mathrm{H}), 7.45(\mathrm{~d}, J=7.5 \mathrm{~Hz}, 2 \mathrm{H}), 7.37-7.32(\mathrm{~m}, 2 \mathrm{H}), 7.31-7.25(\mathrm{~m}, 4 \mathrm{H})$, $7.23(\mathrm{td}, J=8.0,1.0 \mathrm{~Hz}, 1 \mathrm{H}), 7.20-7.11(\mathrm{~m}, 2 \mathrm{H}), 7.05-7.01(\mathrm{~m}, 1 \mathrm{H}), 6.98(\mathrm{~d}, J=8.0 \mathrm{~Hz}, 1 \mathrm{H}), 6.81(\mathrm{~d}, J$ $=8.0 \mathrm{~Hz}, 1 \mathrm{H}), 5.07(\mathrm{~d}, J=16.0 \mathrm{~Hz}, 1 \mathrm{H}), 4.99(\mathrm{~d}, J=15.5 \mathrm{~Hz}, 1 \mathrm{H}), 3.70-3.61(\mathrm{~m}, 1 \mathrm{H}), 3.51-3.43(\mathrm{~m}$, 1H), 2.78 (ddd, $J=14.0,11.5,5.0 \mathrm{~Hz}, 1 \mathrm{H}), 2.55$ (ddd, $J=14.011 .5,4.5 \mathrm{~Hz}, 1 \mathrm{H}) .{ }^{13} \mathbf{C}$ NMR (126 MHz, $\left.\mathrm{CDCl}_{3}\right) \delta 176.23,164.94,141.99,135.96,135.25,129.61,129.02,128.88,128.77,127.93,127.23$, $125.04,123.71,123.02,122.91,121.80,120.26,111.95,110.05,109.77,60.10,45.20\left(\mathrm{~d}, J_{\mathrm{C}-\mathrm{F}}=18.1 \mathrm{~Hz}\right)$, 44.47, 30.74. ${ }^{19} \mathbf{F}$ NMR (471 MHz, $\left.\mathrm{CDCl}_{3}\right) \delta$ 53.38. HRMS (ESI) $\mathrm{m} / z$ : Calcd. for $\mathrm{C}_{26} \mathrm{H}_{23} \mathrm{~N}_{3} \mathrm{O}_{4} \mathrm{FS}$, $[\mathrm{M}+\mathrm{H}]^{+}, 492.1388$; found: 492.1401. $[\alpha]_{\mathrm{D}}{ }^{20}=-4.3\left(\mathrm{c} 1.0, \mathrm{CH}_{2} \mathrm{Cl}_{2}\right)$. Chiral HPLC analysis (Chiral pak AS- $\mathrm{H}, i-\mathrm{PrOH} / n$-hexane $=20: 80$, flow rate $=0.5 \mathrm{~mL} / \mathrm{min}$, wave length $=254 \mathrm{~nm}), \mathrm{t}_{\mathrm{R}}($ minor enantiomer) $=26.424 \mathrm{~min}, \mathrm{t}_{\mathrm{R}}$ (major enantiomer) $=68.562 \mathrm{~min}, 99 \% e e$.

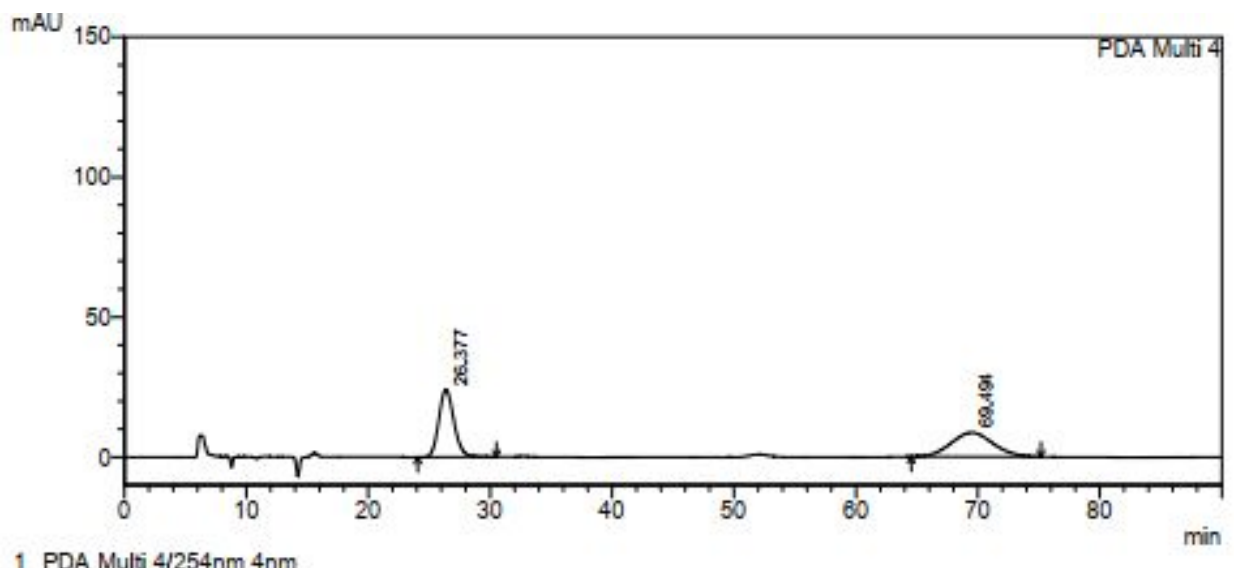

PeakTable

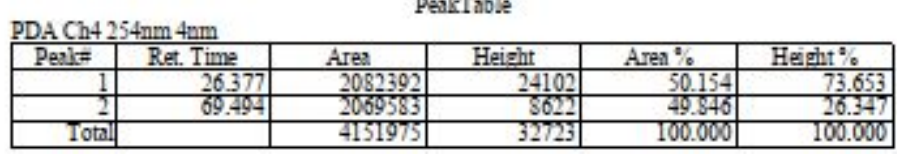




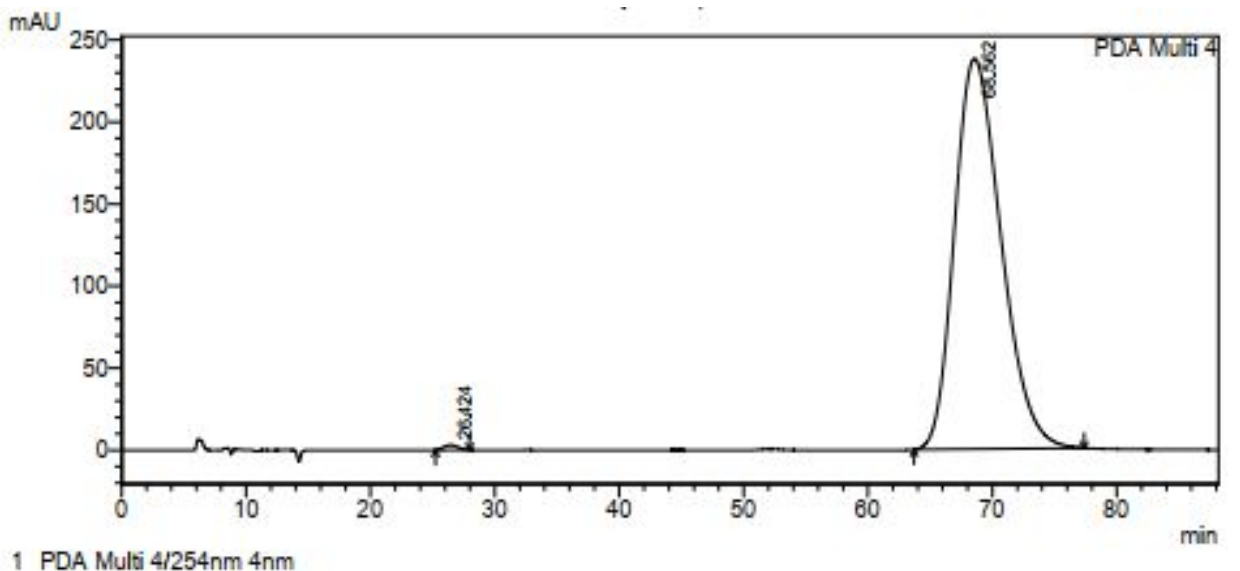

1 PDA Multi $4 / 254 \mathrm{~nm} 4 \mathrm{~nm}$

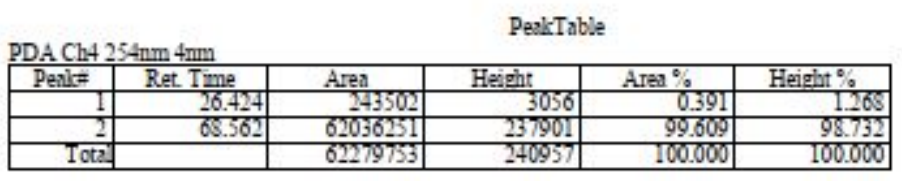

(S)-2-(1-Benzyl-2-oxo-3-(thiophene-2-carboxamido)indolin-3-yl)ethane-1-sulfonyl fluoride (3t)<smiles>O=C(N[C@]1(CCS(=O)(=O)F)C(=O)N(Cc2ccccc2)c2ccccc21)c1cccs1</smiles>

Petroleum ether/ethyl acetate $=8: 1$ was used as the eluent for column chromatography. White solid (44.2 mg, 97\%), m. p. $235.5-236.6{ }^{\circ} \mathrm{C} .{ }^{1} \mathbf{H}$ NMR $\left(400 \mathrm{MHz}, \mathrm{CDCl}_{3}\right) \delta 7.55(\mathrm{~d}, J=3.6 \mathrm{~Hz}, 1 \mathrm{H}), 7.47(\mathrm{~d}$, $J=5.2 \mathrm{~Hz}, 1 \mathrm{H}), 7.42-7.34$ (m, 5H), 7.32-7.27 (m, 1H), 7.25-7.19 (m, 2H), 7.10-7.02 (m, 2H), 6.80 (d, $J$ $=8.0 \mathrm{~Hz}, 1 \mathrm{H}), 5.05(\mathrm{~d}, J=15.6 \mathrm{~Hz}, 1 \mathrm{H}), 4.94(\mathrm{~d}, J=15.6 \mathrm{~Hz}, 1 \mathrm{H}), 3.78-3.67(\mathrm{~m}, 1 \mathrm{H}), 3.50-3.39(\mathrm{~m}$, $1 \mathrm{H}), 2.87-2.76(\mathrm{~m}, 1 \mathrm{H}), 2.56-2.44(\mathrm{~m}, 1 \mathrm{H}) .{ }^{13} \mathbf{C}$ NMR $\left(101 \mathrm{MHz}, \mathrm{CDCl}_{3}\right) \delta$ 175.07, 161.40, 142.10, 137.06, 135.24, 131.36, 129.85, 129.35, 129.01, 128.20, 127.91, 127.86, 127.26, 123.67, 123.09, 110.05, 59.97, $44.95\left(\mathrm{~d}, J_{\mathrm{C}-\mathrm{F}}=18.2 \mathrm{~Hz}\right), 44.43,30.40 .{ }^{19} \mathbf{F}$ NMR $\left(376 \mathrm{MHz}, \mathrm{CDCl}_{3}\right) \delta 53.32$. HRMS (ESI) $\mathrm{m} / \mathrm{z}$ : Calcd. for $\mathrm{C}_{22} \mathrm{H}_{19} \mathrm{~N}_{2} \mathrm{O}_{4} \mathrm{FS}_{2} \mathrm{Na}$, [M+Na] $]^{+}$, 481.0662; found 481.0677. [ $\left.\alpha\right]_{\mathrm{D}}{ }^{20}=+23.7$ (c 1.0, $\mathrm{CH}_{2} \mathrm{Cl}_{2}$ ). Chiral HPLC analysis (Chiral pak AS-H, $i-\mathrm{PrOH} / n$-hexane $=20: 80$, flow rate $=0.5 \mathrm{~mL} / \mathrm{min}$, wave length $=254 \mathrm{~nm}), \mathrm{t}_{\mathrm{R}}($ minor enantiomer $)=18.235 \mathrm{~min}, \mathrm{t}_{\mathrm{R}}($ major enantiomer $)=29.124 \mathrm{~min}, 99 \%$ ee. 


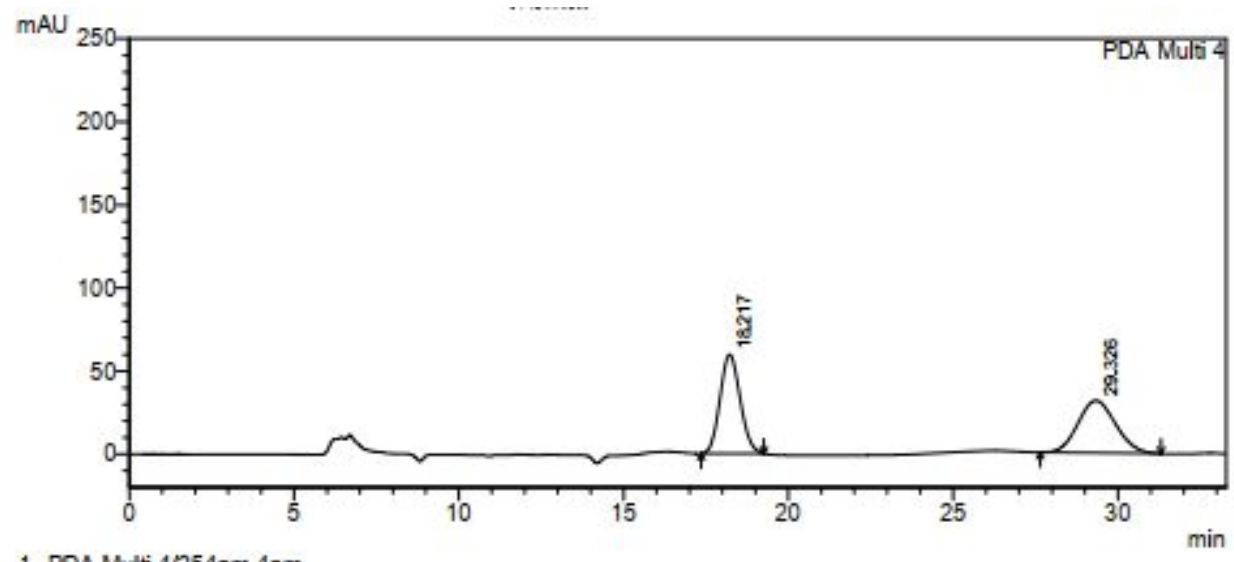

1. PDA Multi 4/254nm 4nm
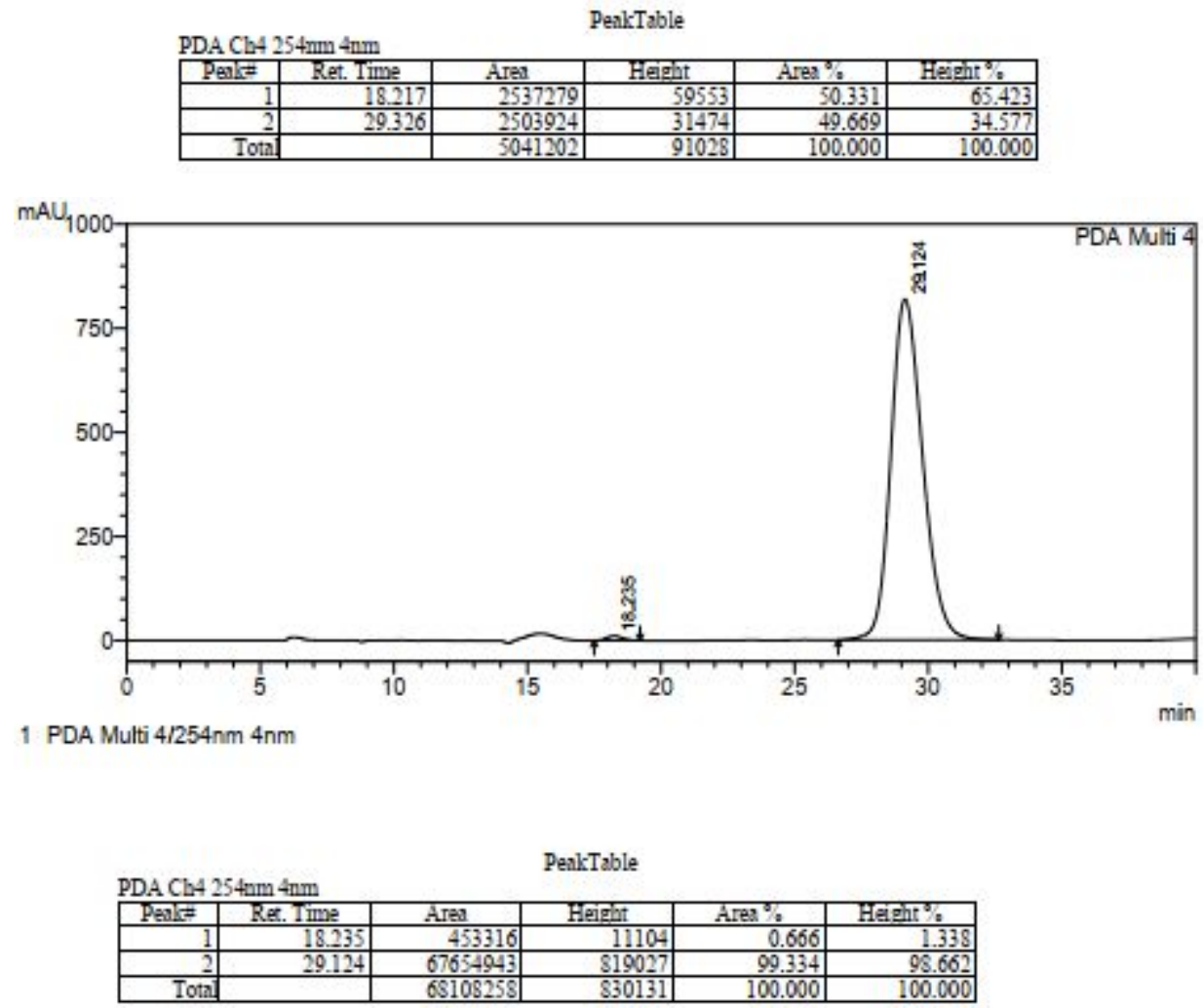

(S)-2-(1-Benzyl-3-(furan-2-carboxamido)-2-oxoindolin-3-yl)ethane-1-sulfonyl fluoride (3u)<smiles>O=C(N[C@]1(CCS(=O)(=O)F)C(=O)N(Cc2ccccc2)c2ccccc21)c1ccco1</smiles>

Petroleum ether/ethyl acetate $=8: 1$ was used as the eluent for column chromatography. White solid (41 mg, 93\%), m. p. $211.7-212.9^{\circ} \mathrm{C}^{1}{ }^{1} \mathbf{H}$ NMR (400 MHz, $\left.\mathrm{CDCl}_{3}\right) \delta$ 7.54-7.43 (m, 2H), 7.43-7.27 (m, 5H), $7.23(\mathrm{dd}, J=8.0,1.2 \mathrm{~Hz}, 1 \mathrm{H}), 7.14-6.97(\mathrm{~m}, 3 \mathrm{H}), 6.79(\mathrm{~d}, J=8.0 \mathrm{~Hz}, 1 \mathrm{H}), 6.51(\mathrm{dd}, J=3.6,1.6 \mathrm{~Hz}, 1 \mathrm{H})$, $5.03(\mathrm{~d}, J=16.0 \mathrm{~Hz}, 1 \mathrm{H}), 4.96(\mathrm{~d}, J=16.0 \mathrm{~Hz}, 1 \mathrm{H}), 3.63-3.44(\mathrm{~m}, 2 \mathrm{H}), 2.91(\mathrm{ddd}, J=13.610 .4,5.2 \mathrm{~Hz}$, 
$1 \mathrm{H}), 2.48$ (ddd, $J=14.0,11.2,4.8 \mathrm{~Hz}, 1 \mathrm{H}) .{ }^{13} \mathbf{C}$ NMR $\left(101 \mathrm{MHz}, \mathrm{CDCl}_{3}\right) \delta 173.42,156.53,145.56$, $143.60,141.16,134.17,128.93,127.98,126.89,126.27,122.64,122.38,114.76,111.43,108.97,58.49$, $43.97\left(\mathrm{~d}, J_{\mathrm{C}-\mathrm{F}}=18.4 \mathrm{~Hz}\right), 43.40,29.47 .{ }^{19} \mathbf{F}$ NMR $\left(376 \mathrm{MHz}, \mathrm{CDCl}_{3}\right) \delta 53.39$. HRMS (ESI) m/z: Calcd. for $\mathrm{C}_{22} \mathrm{H}_{19} \mathrm{~N}_{2} \mathrm{O}_{5} \mathrm{FSNa},[\mathrm{M}+\mathrm{Na}]^{+}, 465.0891$; found: 465.0905. $[\boldsymbol{\alpha}]_{\mathrm{D}}{ }^{20}=+15.8$ (c 1.0, $\mathrm{CH}_{2} \mathrm{Cl}_{2}$ ). Chiral HPLC analysis (Chiral pak AS-H, $i-\mathrm{PrOH} / n$-hexane $=20: 80$, flow rate $=0.5 \mathrm{~mL} / \mathrm{min}$, wave length $=$ $254 \mathrm{~nm}), \mathrm{t}_{\mathrm{R}}($ minor enantiomer $)=19.373 \mathrm{~min}, \mathrm{t}_{\mathrm{R}}($ major enantiomer $)=28.519 \mathrm{~min}, 97 \%$ ee

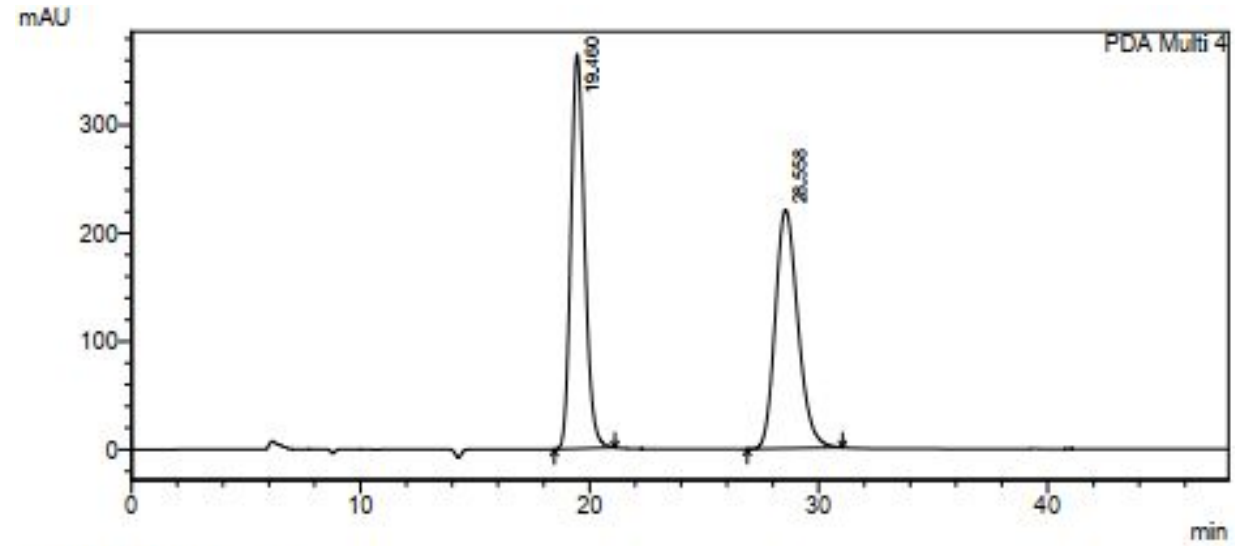

1 PDA Multi $4 / 254 \mathrm{~nm} 4 \mathrm{~nm}$
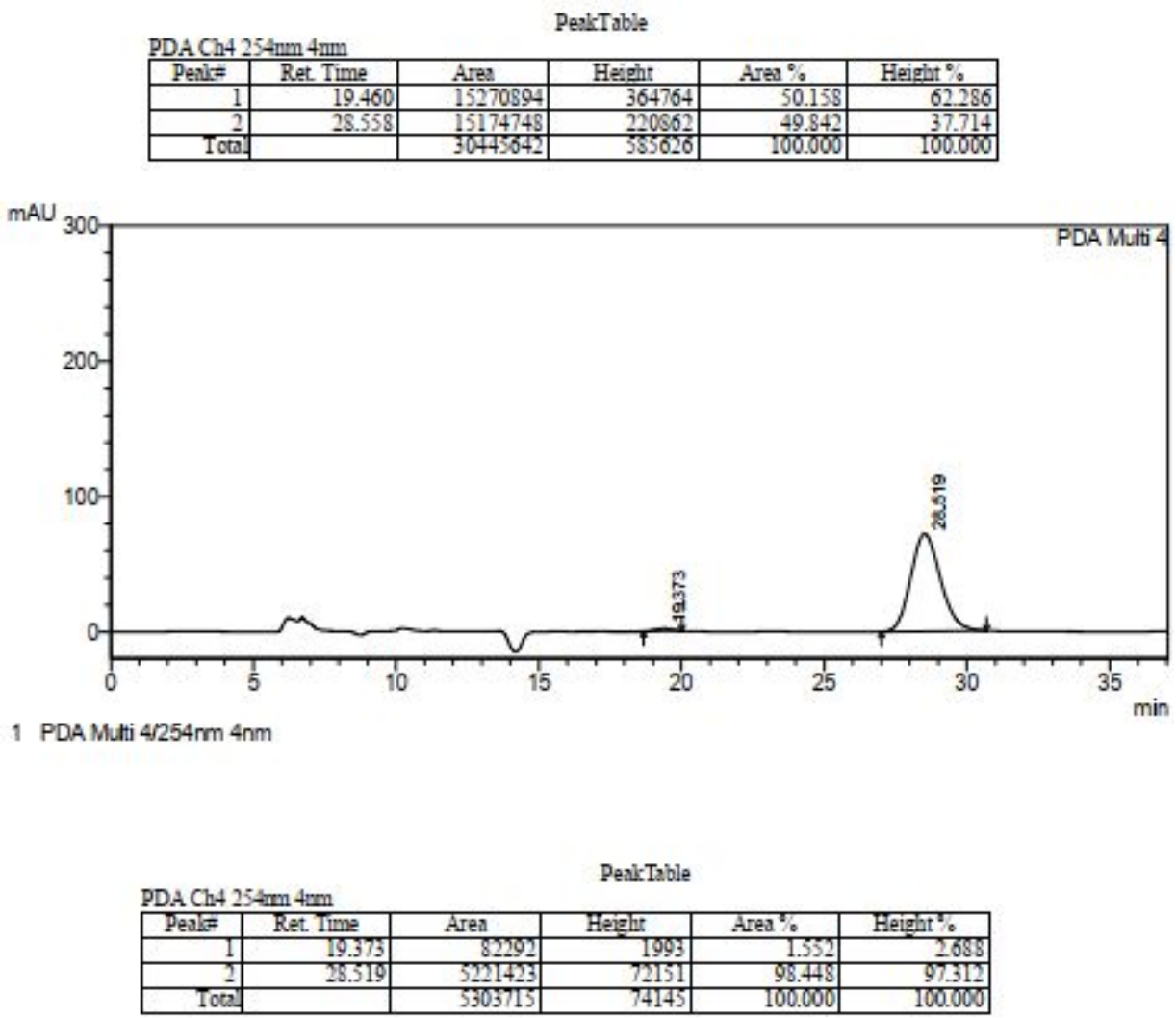

(S)-2-(1-Benzyl-3-(cyclopropanecarboxamido)-2-oxoindolin-3-yl)ethane-1-sulfonyl fluoride (3v)<smiles>O=C(N[C@@]1(CCS(=O)(=O)F)C(=O)N(Cc2ccccc2)c2ccccc21)C1CC1</smiles> 
Petroleum ether/ethyl acetate $=6: 1$ was used as the eluent for column chromatography. White solid (35 mg, 84\%), m. p. $211.9-213^{\circ} \mathrm{C} .{ }^{1} \mathbf{H}$ NMR $\left(400 \mathrm{MHz} \mathrm{CDCl}_{3}\right) \delta 7.39(\mathrm{~d}, J=7.6 \mathrm{~Hz}, 1 \mathrm{H})$, 7.37-7.26 (m, $5 \mathrm{H}), 7.22(\mathrm{td}, J=8.0,1.2 \mathrm{~Hz}, 1 \mathrm{H}), 7.13-7.01(\mathrm{~m}, 1 \mathrm{H}), 6.77(\mathrm{~d}, J=8.0 \mathrm{~Hz}, 1 \mathrm{H}), 6.56(\mathrm{~s}, 1 \mathrm{H}), 5.03(\mathrm{~d}, J$ $=15.6 \mathrm{~Hz}, 1 \mathrm{H}), 4.89(\mathrm{~d}, J=16.0 \mathrm{~Hz}, 1 \mathrm{H}), 3.60-3.49(\mathrm{~m}, 1 \mathrm{H}), 3.48-3.38(\mathrm{~m}, 1 \mathrm{H}), 2.77(\mathrm{ddd}, J=14.0$, 10.8, $4.8 \mathrm{~Hz}, 1 \mathrm{H}), 2.38$ (ddd, $J=14.0,11.2,5.2 \mathrm{~Hz}, 1 \mathrm{H}), 1.51-1.39$ (m, 1H), 1.02-0.92 (m, 1H), 0.91$0.84(\mathrm{~m}, 1 \mathrm{H}), 0.82-0.70(\mathrm{~m}, 2 \mathrm{H}) .{ }^{13} \mathbf{C}$ NMR $\left(101 \mathrm{MHz}, \mathrm{CDCl}_{3}\right) \delta 174.86,173.32,142.03,135.30,129.77$, 128.99, 128.45, 127.92, 127.28, 123.63, 123.34, 109.90, 59.74, 45.06 (d, $\left.J_{\mathrm{C}-\mathrm{F}}=18.3 \mathrm{~Hz}\right), 44.36,30.37$, 14.36, 7.96. ${ }^{19} \mathbf{F}$ NMR $\left(376 \mathrm{MHz}, \mathrm{CDCl}_{3}\right) \delta$ 53.26. HRMS (ESI) $\mathrm{m} / z$ : Calcd. for $\mathrm{C}_{21} \mathrm{H}_{21} \mathrm{~N}_{2} \mathrm{O}_{4} \mathrm{FSNa}$, $[\mathrm{M}+\mathrm{Na}]^{+}$, 439.1098; found: 439.1110. $[\boldsymbol{\alpha}]_{\mathrm{D}}^{20}=-23.9\left(\mathrm{c} 1.0, \mathrm{CH}_{2} \mathrm{Cl}_{2}\right)$. Chiral HPLC analysis (Chiral pak AS-H, $i-\mathrm{PrOH} / n$-hexane $=20: 80$, flow rate $=0.5 \mathrm{~mL} / \mathrm{min}$, wave length $=254 \mathrm{~nm}), \mathrm{t}_{\mathrm{R}}$ (minor enantiomer) $=19.882 \mathrm{~min}, \mathrm{t}_{\mathrm{R}}$ (major enantiomer) $24.210 \mathrm{~min}, 92 \%$ ee.

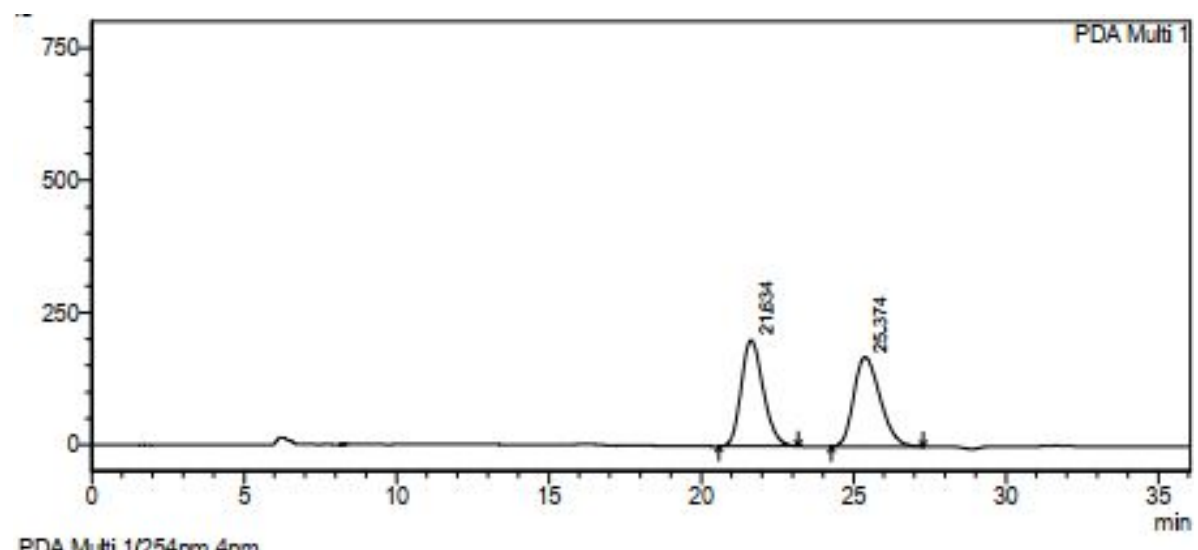

PDA Muti $1 / 254 \mathrm{~mm} 4 \mathrm{~nm}$
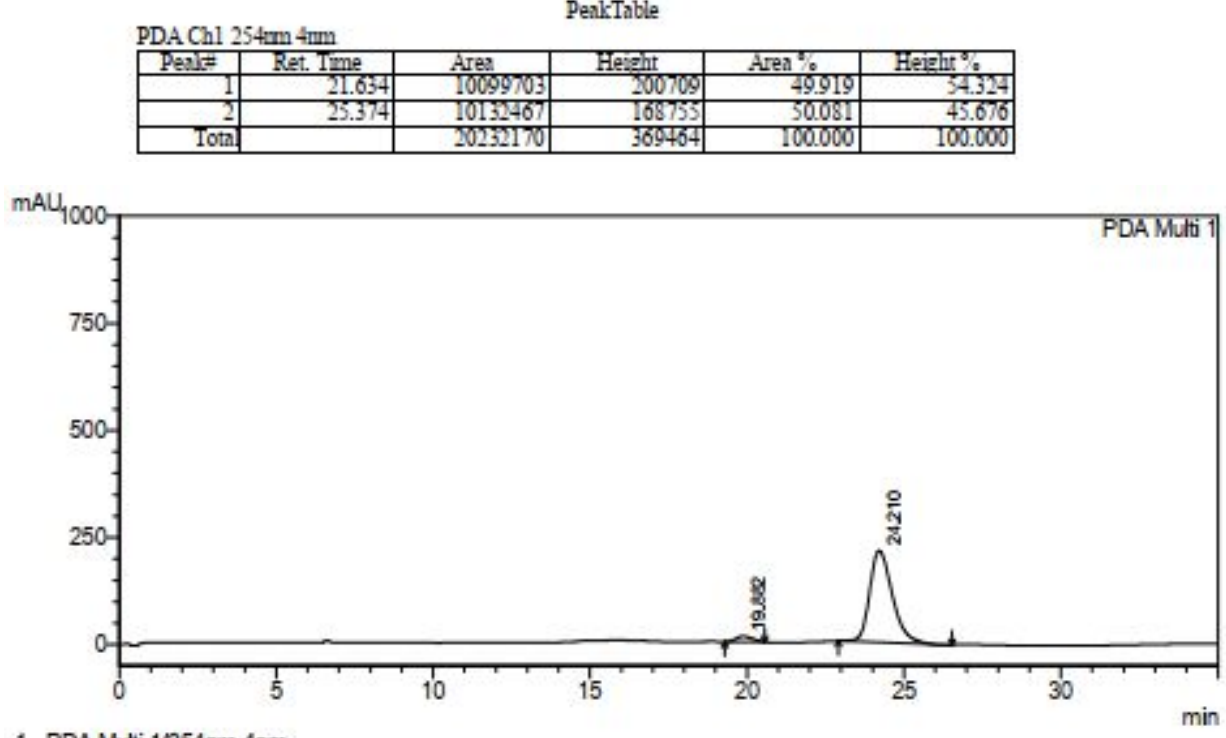

1 PDA Mutti $1 / 254 \mathrm{~mm} 4 \mathrm{~nm}$

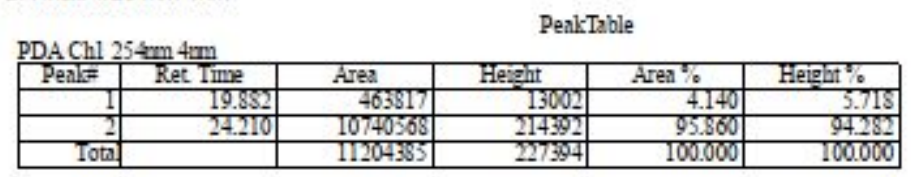

(S)-2-(1-Benzyl-3-(cyclopentanecarboxamido)-2-oxoindolin-3-yl)ethane-1-sulfonyl fluoride (3w) 
<smiles>O=C(NC1(CCS(=O)(=O)F)C(=O)N(c2ccccc2)c2ccccc21)C1CCCC1</smiles>

Petroleum ether/ethyl acetate $=6: 1$ was used as the eluent for column chromatography. White solid (33 mg, 75\%), m. p. ${ }^{163.9-164.4}{ }^{\circ} \mathrm{C} .{ }^{1} \mathbf{H}$ NMR $\left(500 \mathrm{MHz}, \mathrm{CDCl}_{3}\right) \delta$ 7.38-7.32 (m, $\left.5 \mathrm{H}\right), 7.30-7.26(\mathrm{~m}, 1 \mathrm{H})$, $7.22(\mathrm{td}, J=8.0,1.5 \mathrm{~Hz}, 1 \mathrm{H}), 7.06(\mathrm{td}, J=7.5,0.5 \mathrm{~Hz}, 1 \mathrm{H}), 6.77(\mathrm{~d}, J=8.0 \mathrm{~Hz}, 1 \mathrm{H}), 6.38(\mathrm{~s}, 1 \mathrm{H}), 5.04$ $(\mathrm{d}, J=15.5 \mathrm{~Hz}, 1 \mathrm{H}), 4.88(\mathrm{~d}, J=15.5 \mathrm{~Hz}, 1 \mathrm{H}), 3.60-3.47(\mathrm{~m}, 1 \mathrm{H}), 3.45-3.34(\mathrm{~m}, 1 \mathrm{H}), 2.73(\mathrm{ddd}, J=$ $14.0,10.5,5.0 \mathrm{~Hz}, 1 \mathrm{H}), 2.65-2.57(\mathrm{~m}, 1 \mathrm{H}), 2.35$ (ddd, $J=14.0,10.5,5.0 \mathrm{~Hz}, 1 \mathrm{H}), 1.88-1.81(\mathrm{~m}, 2 \mathrm{H})$, 1.80-1.74 (m, 1H), 1.70-1.65 (m, 3H), 1.59-1.51 (m, 2H). ${ }^{13} \mathbf{C}$ NMR (126 MHz, $\left.\mathrm{CDCl}_{3}\right) \delta 175.99,174.90$, $142.14,135.38,129.74,129.00,128.50,127.91,127.31,123.59,122.97,109.91,59.50,45.00\left(\mathrm{~d}, J_{\mathrm{C}-\mathrm{F}}=\right.$ $18.2 \mathrm{~Hz}), 44.83,44.38,30.48,30.33,29.93,26.03,25.96 .{ }^{19} \mathbf{F}$ NMR (471 MHz, $\left.\mathrm{CDCl}_{3}\right) \delta 53.26$. HRMS (ESI) $m / z$ : Calcd. for $\mathrm{C}_{23} \mathrm{H}_{25} \mathrm{~N}_{2} \mathrm{O}_{4} \mathrm{FSNa}$, [M+Na] $]^{+}$, 467.1411; found: 467.1417. [a $]_{\mathrm{D}}^{20}=-15.9$ (c 1.0, $\mathrm{CH}_{2} \mathrm{Cl}_{2}$ ). Chiral HPLC analysis (Chiral pak AS-H, $i-\mathrm{PrOH} / n$-hexane $=20: 80$, flow rate $=0.5 \mathrm{~mL} / \mathrm{min}$, wave length $=254 \mathrm{~nm}), t_{\mathrm{R}}($ minor enantiomer $)=29.540 \mathrm{~min}, \mathrm{t}_{\mathrm{R}}($ major enantiomer $)=37.492 \mathrm{~min}, 95 \%$ ee.

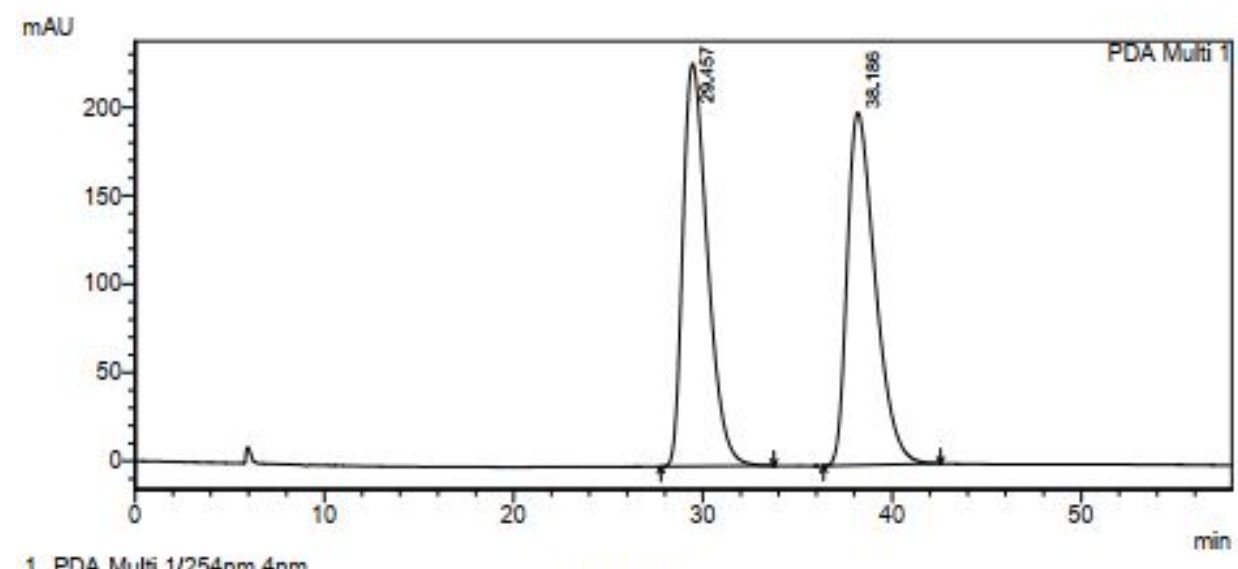

1 PDA Multi $1 / 254 \mathrm{~nm} 4 \mathrm{~nm}$

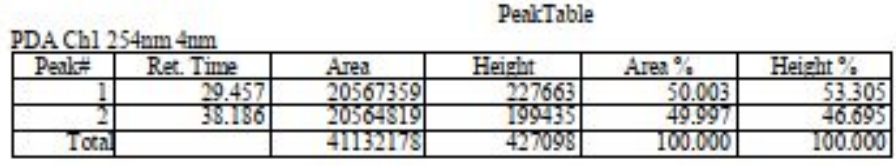




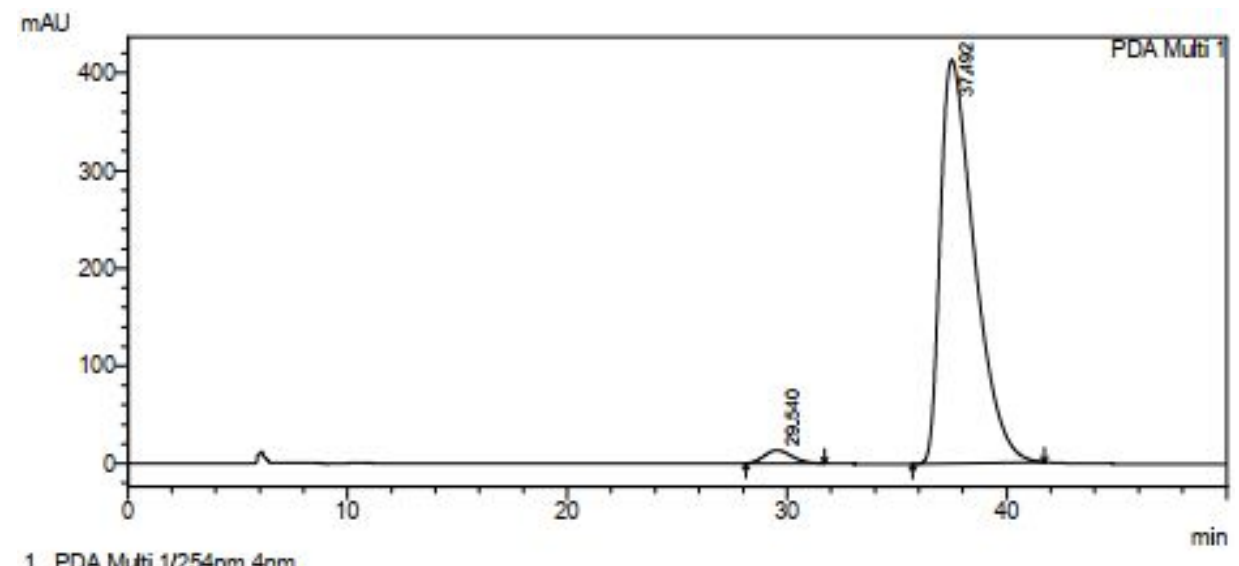

1 PDA Mutti $1 / 254 \mathrm{~nm} 4 \mathrm{~nm}$

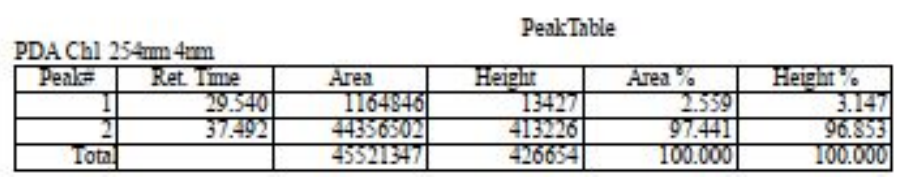

(S)-2-(1-Benzyl-2-oxo-3-(tetrahydro-2H-pyran-4-carboxamido)indolin-3-yl)ethane-1-sulfonyl fluoride (3x)<smiles>CC(C)(C)N1C(=O)[C@@H](CCS(=O)(=O)F)c2ccccc21</smiles>

Petroleum ether/ethyl acetate $=6: 1$ was used as the eluent for column chromatography. White solid (22 mg, 46\%), m. p. $239.8-240.9^{\circ} \mathrm{C} .{ }^{1} \mathbf{H}$ NMR $\left(500 \mathrm{MHz}, \mathrm{CDCl}_{3}\right) \delta$ 7.38-7.32 (m, 5H), 7.31-7.27 (m, $\left.1 \mathrm{H}\right)$, $7.23(\mathrm{td}, J=8.0,1.5 \mathrm{~Hz}, 1 \mathrm{H}), 7.07(\mathrm{td}, J=7.5,0.5 \mathrm{~Hz}, 1 \mathrm{H}), 6.78$ (d, $J=8.0 \mathrm{~Hz}, 1 \mathrm{H}), 6.47$ (s, 1H), 5.01 (d, $J=15.5 \mathrm{~Hz}, 1 \mathrm{H}), 4.92(\mathrm{~d}, J=15.5 \mathrm{~Hz}, 1 \mathrm{H}), 4.02-3.95(\mathrm{~m}, 2 \mathrm{H}), 3.63-3.55(\mathrm{~m}, 1 \mathrm{H}), 3.43-3.34(\mathrm{~m}, 3 \mathrm{H})$, 2.72 (ddd, $J=14.5,10.0,5.0 \mathrm{~Hz}, 1 \mathrm{H}), 2.44-2.33(\mathrm{~m}, 2 \mathrm{H}), 1.82-1.69(\mathrm{~m}, 5 \mathrm{H}) .{ }^{13} \mathbf{C}$ NMR $(126 \mathrm{MHz}$, $\left.\mathrm{CDCl}_{3}\right) \delta 174.72,173.95,142.13,135.25,129.88,129.01,128.27,127.95,127.30,123.65,122.88$, $110.00,67.05,67.02,59.39,44.91\left(\mathrm{~d}, J_{\mathrm{C}-\mathrm{F}}=18.2 \mathrm{~Hz}\right), 44.40,41.46,30.28,29.10,28.80 .{ }^{19} \mathbf{F}$ NMR $(471$ $\mathrm{MHz}, \mathrm{CDCl}_{3}$ ) $\delta$ 53.28. HRMS (ESI) $m / z$ : Calcd. for $\mathrm{C}_{23} \mathrm{H}_{25} \mathrm{~N}_{2} \mathrm{O}_{5} \mathrm{FSNa}$, [M+Na] $]^{+}, 483.1360$; found: 483.1363. $[\boldsymbol{\alpha}]_{\mathrm{D}}{ }^{20}=+9.8\left(\mathrm{c} 1.0, \mathrm{CH}_{2} \mathrm{Cl}_{2}\right)$. Chiral HPLC analysis (Chiral pak AS-H, $i-\mathrm{PrOH} / n$-hexane $=$ $20: 80$, flow rate $=0.5 \mathrm{~mL} / \mathrm{min}$, wave length $=254 \mathrm{~nm}), \mathrm{t}_{\mathrm{R}}($ minor enantiomer $)=23.256 \mathrm{~min}, \mathrm{t}_{\mathrm{R}}$ (major enantiomer) $=28.497 \mathrm{~min}, 94 \%$ ee . 


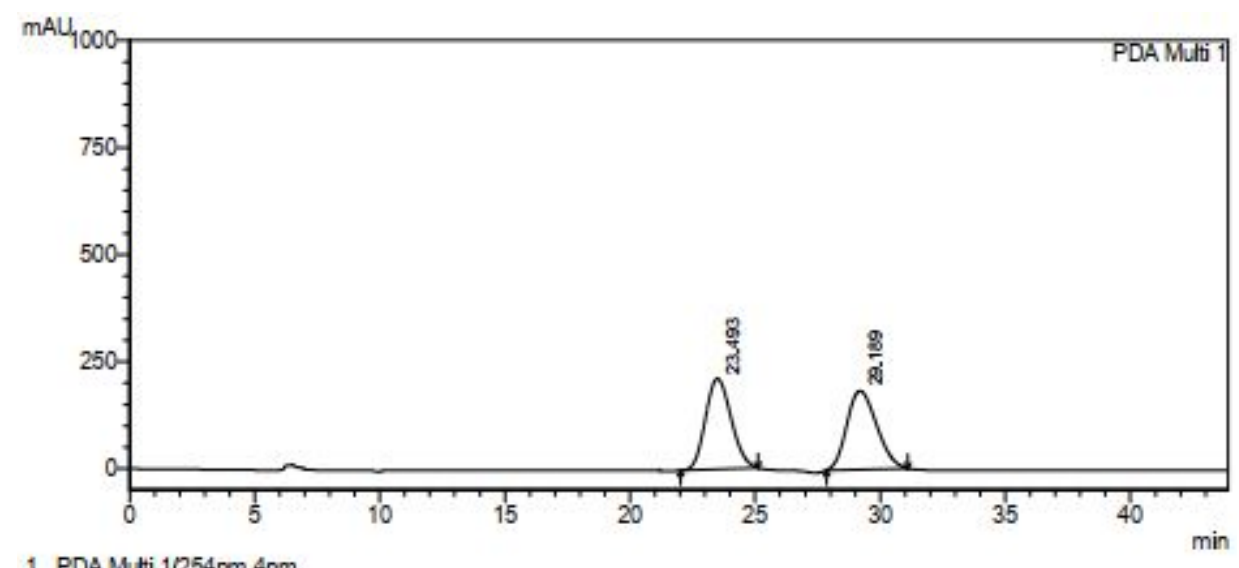

1 PDA Mutii $1 / 254 \mathrm{~mm} 4 \mathrm{~nm}$
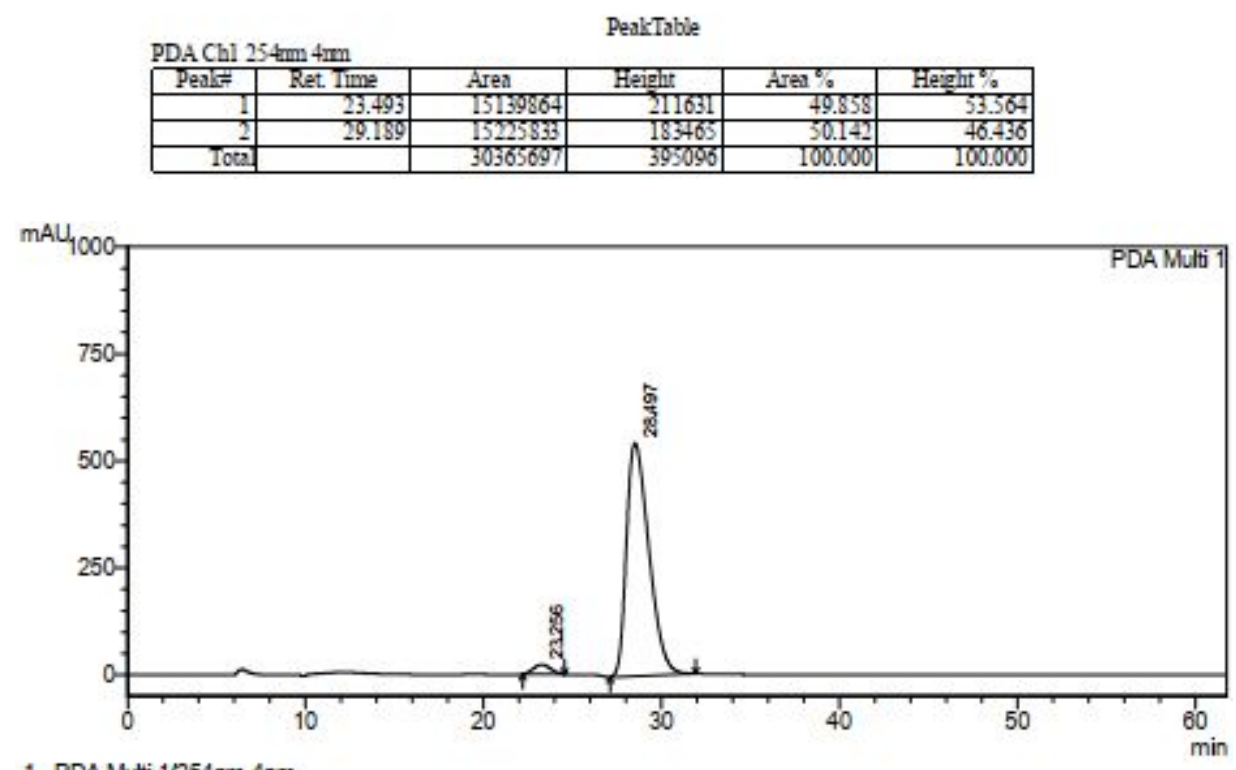

1 PDA Mutti $1 / 254 \mathrm{rm}$ 4nm

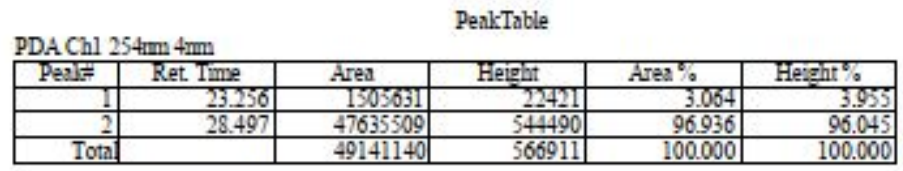

Ethyl (S)-(1-benzyl-3-(2-(fluorosulfonyl)ethyl)-2-oxoindolin-3-yl)carbamate (3y)<smiles>CCOC(=O)N[C@@]1(CCS(=O)(=O)OCC)C(=O)N(Cc2ccccc2)c2ccccc21</smiles>

3y

Petroleum ether/ethyl acetate $=8: 1$ was used as the eluent for column chromatography. White solid (29 $\mathrm{mg}, 70 \%)$, m. p. $207.9-208.7^{\circ} \mathrm{C} .{ }^{1} \mathbf{H}$ NMR $\left(500 \mathrm{MHz}, \mathrm{CDCl}_{3}\right) \delta 7.41$ (d, $\left.J=7.5 \mathrm{~Hz}, 1 \mathrm{H}\right), 7.37-7.28(\mathrm{~m}$, $5 \mathrm{H}), 7.28-7.23(\mathrm{~m}, 2 \mathrm{H}), 7.12-7.07(\mathrm{~m}, 1 \mathrm{H}), 6.79(\mathrm{~d}, J=7.5 \mathrm{~Hz}, 1 \mathrm{H}), 5.67(\mathrm{~s}, 1 \mathrm{H}), 4.99-4.90(\mathrm{~m}, 2 \mathrm{H})$, 4.09-3.96 (m, 2H), 3.55-3.47 (m, 2H), 2.76-2.67 (m, 1H), 2.39-2.31 (m, 1H), $1.12(\mathrm{~s}, 3 \mathrm{H}) .{ }^{13} \mathbf{C}$ NMR $\left(126 \mathrm{MHz}, \mathrm{CDCl}_{3}\right) \delta 174.86,154.85,142.01,135.18,129.92,128.97,127.97,127.36,123.63,123.36$, $109.90,61.75,59.62,45.07\left(\mathrm{~d}, J_{\mathrm{C}-\mathrm{F}}=18.5 \mathrm{~Hz}\right), 44.33,30.62,14.26 .{ }^{19} \mathbf{F}$ NMR $\left(471 \mathrm{MHz}, \mathrm{CDCl}_{3}\right) \delta$ 


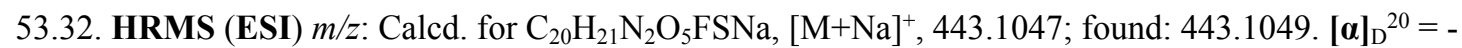
$16.9\left(\mathrm{c} 1.0, \mathrm{CH}_{2} \mathrm{Cl}_{2}\right)$. Chiral HPLC analysis (Chiral pak AS-H, $i-\mathrm{PrOH} / n$-hexane $=20: 80$, flow rate $=$ $0.5 \mathrm{~mL} / \mathrm{min}$, wave length $=254 \mathrm{~nm}), \mathrm{t}_{\mathrm{R}}($ minor enantiomer $)=12.691 \mathrm{~min}, \mathrm{t}_{\mathrm{R}}($ major enantiomer $)=15.989$ $\min , 90 \%$ ee.

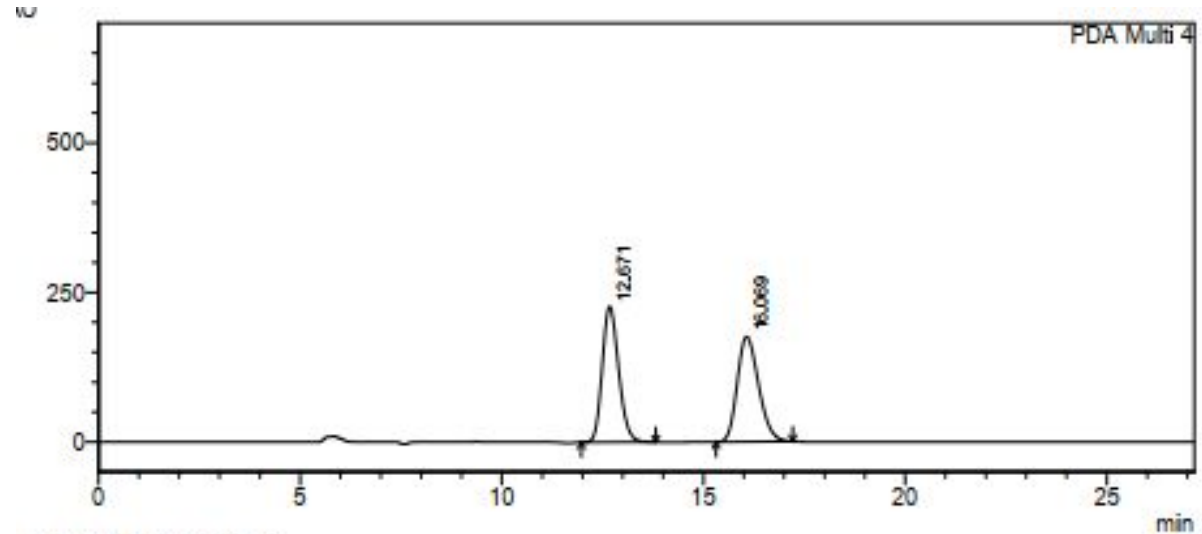

PDA Multi 4/254nm 4nm

PeakTable

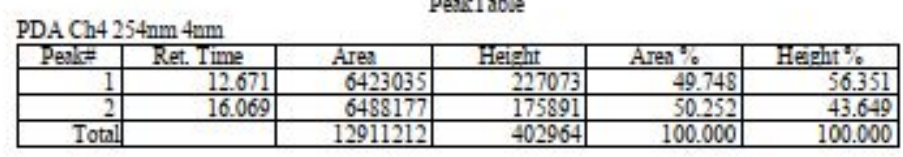

MAU

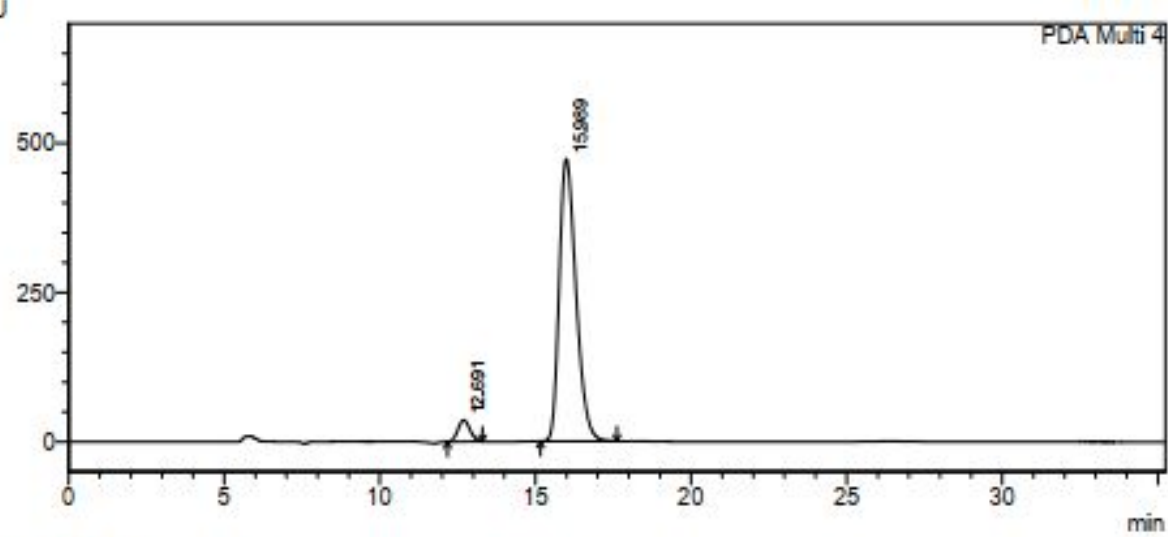

1 PDA Multi $4 / 254 \mathrm{~nm} 4 \mathrm{~nm}$

\begin{tabular}{|c|c|c|c|c|c|}
\hline \multicolumn{6}{|c|}{ PenkTable } \\
\hline Peall:F & Res. Time & Aren & Height & rea \% & Hergit \% \\
\hline & 12.691 & 977959 & 36143 & 5.233 & 7.100 \\
\hline & 15.989 & 17709398 & 472901 & 94.767 & 92.900 \\
\hline Tot: & & 18687857 & 509044 & 100.000 & 00.000 \\
\hline
\end{tabular}




\section{Typical procedures and characterization data for compounds 5}

\subsection{General procedure for the synthesis of compounds 5}<smiles>[R1]N1C(=O)C(=CC#[R])c2cc[R]#cc21</smiles>

$3 a, 3 m, 3 r$<smiles></smiles>

$5 a, 5 m, 5 r$

To a stirred solution of $3(0.1 \mathrm{mmol})$ in $\mathrm{CH}_{2} \mathrm{Cl}_{2}(1.0 \mathrm{~mL})$ was added DBU $(3 \mathrm{mg}, 0.02 \mathrm{mmol})$. The mixture was stirred at $\mathrm{rt}$ for $12 \mathrm{~h}$. After the completion of the reaction (monitored by TLC), the mixture was concentrated under vacuum. The residue was purified by flash chromatography over silica gel to afford the products 5 .

\subsection{General procedure for the synthesis of racemic products 5}<smiles>[R]C(=O)NC1C(=[O+])N([R1])c2cc([R])ccc21</smiles>

$1 \mathrm{a}, 1 \mathrm{~m}, 1 \mathrm{r}$

2a<smiles>[R]C(=O)N1C2(CCS1(=O)=O)C(=O)N([R])c1ccccc12</smiles>

$5 \mathrm{a}, 5 \mathrm{~m}, 5 \mathrm{r}$

A solution of 3-benzamido-2-oxindole 1 ( $0.1 \mathrm{mmol})$, ethylene sulfonyl fluoride $\mathbf{2 a}(13.2 \mathrm{mg}, 0.12 \mathrm{mmol})$, $\mathrm{NaHCO}_{3}(16.8 \mathrm{mg}, 0.2 \mathrm{mmol})$ and DBU $(3 \mathrm{mg}, 0.02 \mathrm{mmol})$ in $\mathrm{CH}_{2} \mathrm{Cl}_{2}(1.0 \mathrm{~mL})$ was stirred at $\mathrm{rt}$ for 12 h. After the solvent was evaporated under vacuum, the residue was purified by flash column chromatography over silica gel (petroleum ether/EtOAc $=8: 1$ ) to afford the racemic products 5 .

\subsection{Scale-up synthesis of compound $5 \mathrm{a}$}<smiles>O=C(N[C@]1(CCS(=O)(=O)F)C(=O)N(Cc2ccccc2)c2ccccc21)c1ccccc1</smiles>

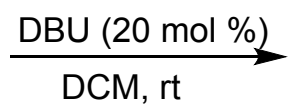

$\mathrm{Bn}$<smiles>O=C1C(N(C(=O)c2ccccc2)S(=O)(=O)CCc2ccccc2)c2ccccc2N1Cc1ccccc1</smiles>

$\mathrm{Bn}$

To a stirred solution of 3a (678 mg, $1.5 \mathrm{mmol})$ in $\mathrm{CH}_{2} \mathrm{Cl}_{2}(15 \mathrm{~mL})$ was added DBU (46 mg, $\left.0.3 \mathrm{mmol}\right)$. The mixture was stirred at $\mathrm{rt}$ for $12 \mathrm{~h}$. After the completion of the reaction (monitored by TLC), the mixture was concentrated under vacuum. The residue was purified by flash chromatography over silica gel (Petroleum ether/ethyl acetate $=8: 1)$ to afford the product $\mathbf{5 a}$ as a white solid $(637 \mathrm{mg}, 98 \%)$.

\subsection{Characterizations of products 5}

(S)-2'-Benzoyl-1-benzylspiro[indoline-3,3'-isothiazolidin]-2-one 1',1'-dioxide (5a)<smiles>O=C(c1ccccc1)N1C2(CCS1(=O)=O)C(=O)Nc1ccccc12</smiles>

$5 \mathrm{a} \mathrm{Bn}$ 
Petroleum ether/ethyl acetate $=8: 1$ was used as the eluent for column chromatography. White solid (44.6 mg, 99\%), m.p. 194.5-195.9 ${ }^{\circ} \mathrm{C} .{ }^{1} \mathbf{H}$ NMR $\left(500 \mathrm{MHz}, \mathrm{CDCl}_{3}\right) \delta 7.85(\mathrm{~d}, J=7.5 \mathrm{~Hz}, 2 \mathrm{H}), 7.59-7.52$ (m, 1H), 7.50-7.40 (m, 5H), $7.36(\mathrm{t}, J=7.5 \mathrm{~Hz}, 2 \mathrm{H}), 7.32-7.26(\mathrm{~m}, 1 \mathrm{H}), 7.24-7.18(\mathrm{~m}, 1 \mathrm{H}), 7.06(\mathrm{t}, J=$ $7.5 \mathrm{~Hz}, 1 \mathrm{H}), 6.72(\mathrm{~d}, J=8.0 \mathrm{~Hz}, 1 \mathrm{H}), 5.05-4.92(\mathrm{~m}, 2 \mathrm{H}), 4.11(\mathrm{td}, J=13.0,6.5 \mathrm{~Hz}, 1 \mathrm{H}), 3.47(\mathrm{dd}, J=$ $12.0,5.5 \mathrm{~Hz}, 1 \mathrm{H}), 2.80(\mathrm{td}, J=14.0,5.5 \mathrm{~Hz}, 1 \mathrm{H}), 2.62(\mathrm{dd}, J=13.5,6.0 \mathrm{~Hz}, 1 \mathrm{H}) .{ }^{13} \mathbf{C ~ N M R}(126 \mathrm{MHz}$, $\left.\mathrm{CDCl}_{3}\right) \delta 174.65,167.79,142.84,135.39,133.46,132.96,130.23,128.91,128.77,128.38,127.78$, 127.31, 126.96, 123.53, 122.29, 109.94, 65.68, 47.14, 44.35, 29.66. HRMS (ESI) m/z: Calcd. for

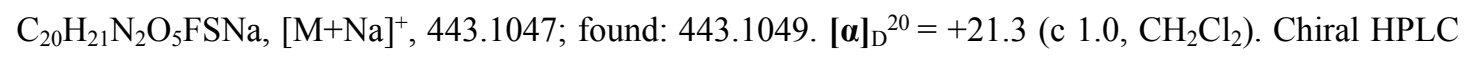
analysis (Chiral pak OD-H, $i-\mathrm{PrOH} / n$-hexane $=20: 80$, flow rate $=1.0 \mathrm{~mL} / \mathrm{min}$, wave length $=254 \mathrm{~nm}$ ), $\mathrm{t}_{\mathrm{R}}($ major enantiomer $)=15.382 \mathrm{~min}, \mathrm{t}_{\mathrm{R}}($ minor enantiomer $)=18.639 \mathrm{~min}, 99 \%$ ee.

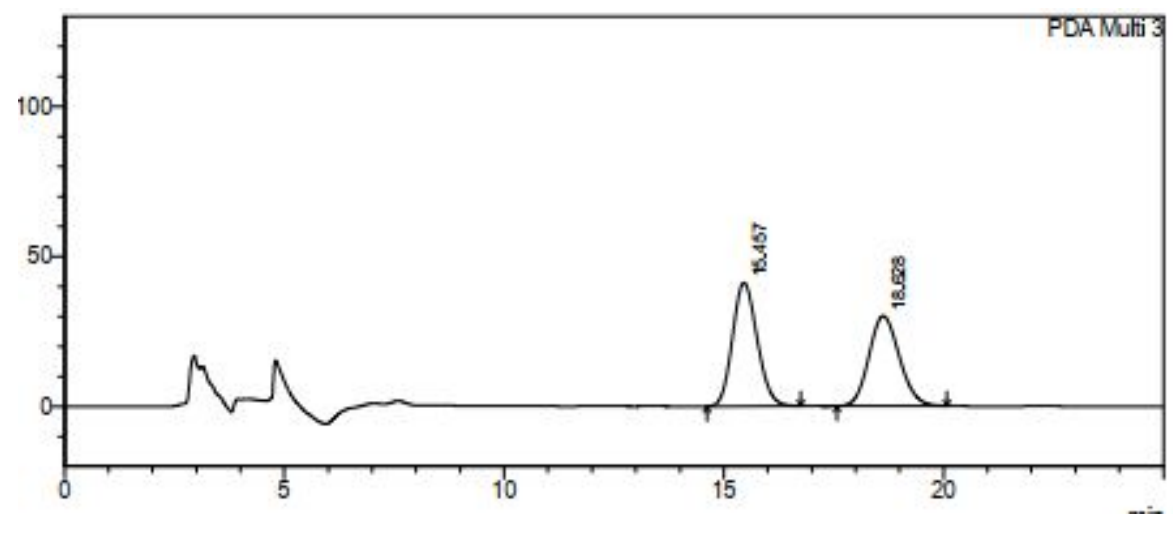

1 PDA Muti $3 / 254 \mathrm{~mm} 4 \mathrm{~nm}$
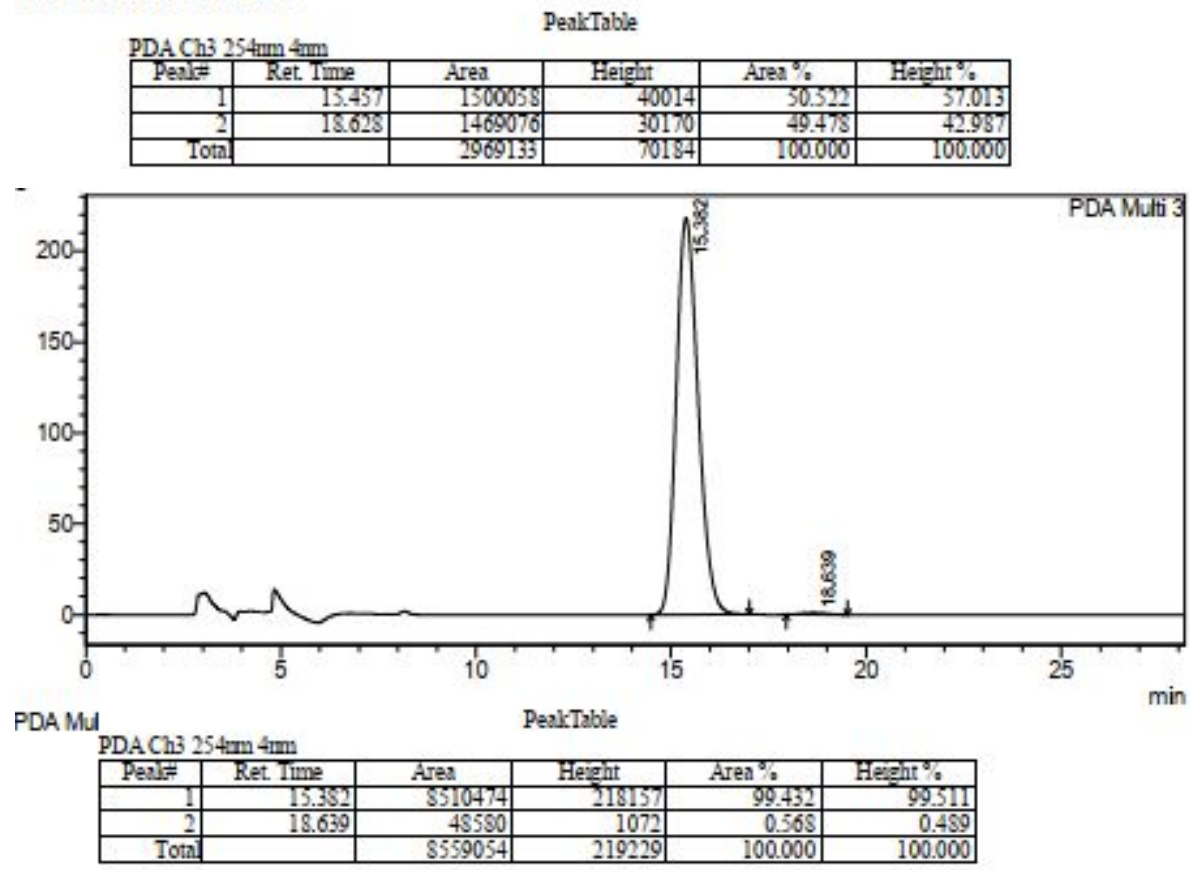

(S)-2'-Benzoyl-1-benzyl-7-fluorospiro[indoline-3,3'-isothiazolidin]-2-one 1',1'-dioxide (5m) 
<smiles>O=C(c1ccccc1)n1c(=O)n(Cc2ccccc2)c2c(F)cccc21</smiles>

Petroleum ether/ethyl acetate $=8: 1$ was used as the eluent for column chromatography. White solid (46.5 mg, 99\%), m. p. $183.5-184.7^{\circ} \mathrm{C} .{ }^{1} \mathbf{H}$ NMR $\left(500 \mathrm{MHz}, \mathrm{CDCl}_{3}\right) \delta 7.85$ (d, J=7.5 Hz, 2H), 7.60-7.54 (m, 1H), 7.49-7.43 (m, 4H), 7.38-7.32( m, 2H), 7.32-7.27 (m, 1H), 7.23 -7.18 (m, 1H), 7.05-6.98 (m, 2H), 5.20-5.07 (m, 2H), 4.16-4.03 (m, 1H), 3.47 (dd, $J=12.0,5.5 \mathrm{~Hz}, 1 \mathrm{H}), 2.76(\mathrm{td}, J=14.0,5.5 \mathrm{~Hz}$, $1 \mathrm{H}), 2.59$ (dd, $J=14.0,6.5 \mathrm{~Hz}, 1 \mathrm{H}) .{ }^{13} \mathbf{C}$ NMR $\left(126 \mathrm{MHz}, \mathrm{CDCl}_{3}\right) \delta 174.33,167.75,147.50$ (d, $J_{\mathrm{C}-\mathrm{F}}=$ $246.1 \mathrm{~Hz}), 136.67,133.18,133.08,129.89\left(\mathrm{~d}, J_{\mathrm{C}-\mathrm{F}}=3.5 \mathrm{~Hz}\right), 129.54\left(\mathrm{~d}, J_{\mathrm{C}-\mathrm{F}}=9.3 \mathrm{~Hz}\right), 128.73,128.66$, 128.42, 127.70, 127.48, $124.33\left(\mathrm{~d}, J_{\mathrm{C}-\mathrm{F}}=6.5 \mathrm{~Hz}\right), 118.47\left(\mathrm{~d}, J_{\mathrm{C}-\mathrm{F}}=19.7 \mathrm{~Hz}\right), 118.12\left(\mathrm{~d}, J_{\mathrm{C}-\mathrm{F}}=3.2 \mathrm{~Hz}\right)$, 65.57, 47.02, 45.90 (d, $J_{\text {C-F }}=4.5 \mathrm{~Hz}$ ), 29.65. HRMS (ESI) $\mathrm{m} / z$ : Calcd. for $\mathrm{C}_{20} \mathrm{H}_{21} \mathrm{~N}_{2} \mathrm{O}_{5} \mathrm{FSNa}$, [M+Na] 443.1047; found: 443.1049. $[\alpha]_{\mathrm{D}}{ }^{20}=+13.2\left(\mathrm{c} 1.0, \mathrm{CH}_{2} \mathrm{Cl}_{2}\right)$. Chiral HPLC analysis (Chiral pak AS-H, $i-$ $\mathrm{PrOH} / n$-hexane $=20: 80$, flow rate $=0.5 \mathrm{~mL} / \mathrm{min}$, wave length $=254 \mathrm{~nm}), \mathrm{t}_{\mathrm{R}}($ minor enantiomer $)=$ $15.367 \mathrm{~min}, \mathrm{t}_{\mathrm{R}}$ (major enantiomer) $=12.604 \mathrm{~min}, 99 \%$ ee.
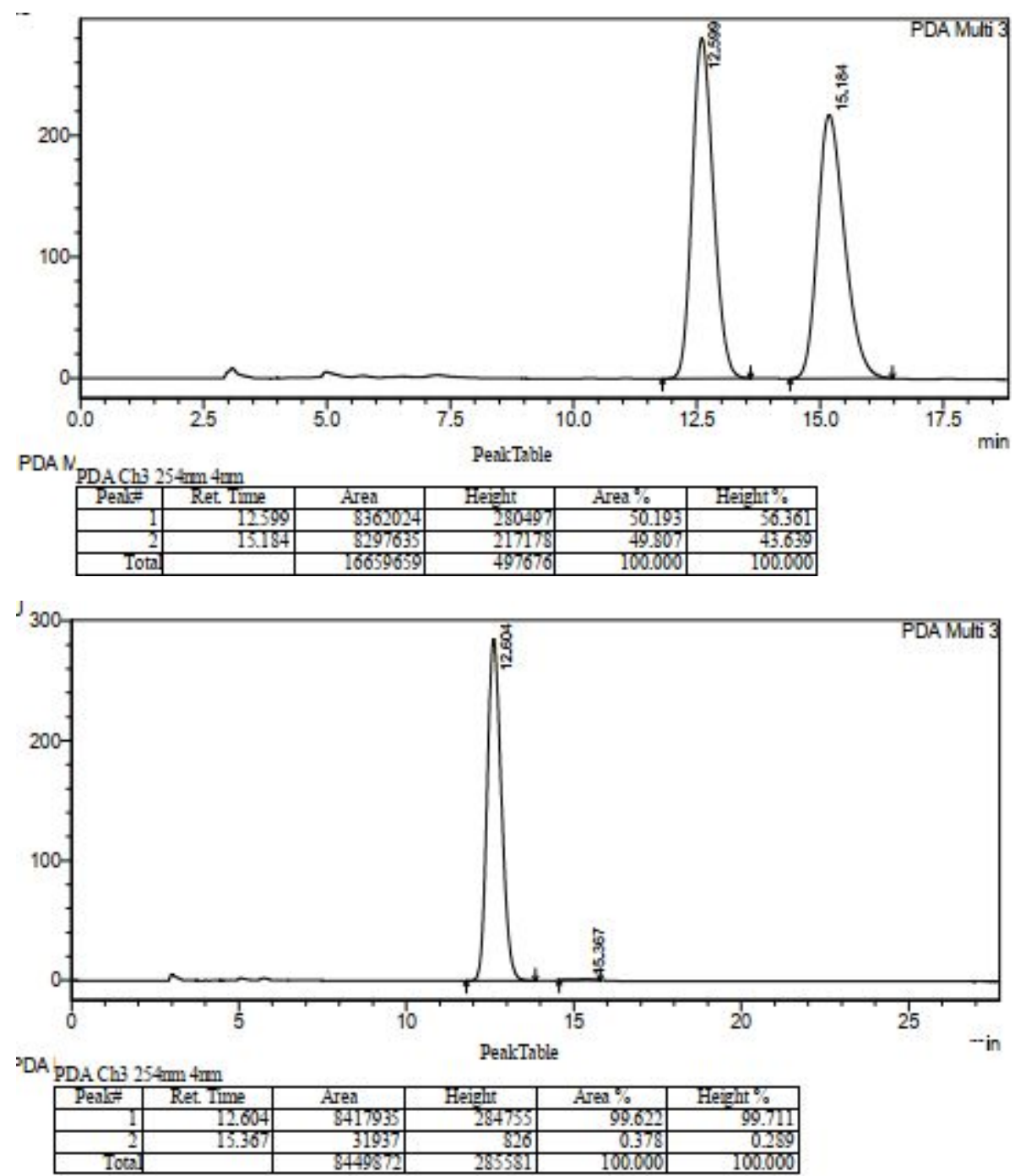
<smiles>O=C1[C@@H]2c3ccccc3N(Cc3ccccc3)[C@H](CC(=O)N2C(=O)c2ccc3ccccc3c2)N1Cc1ccccc1</smiles>

Petroleum ether/ethyl acetate $=6: 1$ was used as the eluent for column chromatography. White solid (46.5 mg, 99\%), m. p. 198.5-199.6 ${ }^{\circ} \mathrm{C} .{ }^{1} \mathbf{H}$ NMR (500 MHz, $\left.\mathrm{CDCl}_{3}\right) \delta 8.48(\mathrm{~s}, 1 \mathrm{H}), 7.98(\mathrm{~d}, J=8.0 \mathrm{~Hz}$, $1 \mathrm{H}), 7.91-7.82(\mathrm{~m}, 3 \mathrm{H}), 7.61-7.52(\mathrm{~m}, 2 \mathrm{H}), 7.51-7.43(\mathrm{~m}, 3 \mathrm{H}), 7.40-7.34(\mathrm{~m}, 2 \mathrm{H}), 7.33-7.27(\mathrm{~m}, 1 \mathrm{H})$, 7.25-7.20 (m, 1H), 7.09 (t, $J=7.5 \mathrm{~Hz}, 1 \mathrm{H}), 6.74(\mathrm{~d}, J=8.0 \mathrm{~Hz}, 1 \mathrm{H}), 5.03(\mathrm{~s}, 2 \mathrm{H}), 4.20-4.10(\mathrm{~m}, 1 \mathrm{H})$, $3.50(\mathrm{dd}, J=12.0,5.5 \mathrm{~Hz}, 1 \mathrm{H}), 2.85(\mathrm{td}, J=14.0,6.0 \mathrm{~Hz}, 1 \mathrm{H}), 2.65(\mathrm{dd}, J=14.0,6.5 \mathrm{~Hz}, 1 \mathrm{H}) .{ }^{13} \mathbf{C} \mathbf{N M R}$ $\left(126 \mathrm{MHz}, \mathrm{CDCl}_{3}\right) \delta 174.72,167.92,142.86,135.45,135.42,132.17,130.64,130.26,130.21,129.50$, $128.93,128.42$, 128.36, 127.88, 127.79, 127.33, 126.98, 126.86, 124.62, 123.56, 122.37, 109.95, 65.82, 47.18, 44.38, 29.71. HRMS (ESI) $m / z$ : Calcd. for $\mathrm{C}_{30} \mathrm{H}_{24} \mathrm{~N}_{2} \mathrm{O}_{4} \mathrm{SNa}$, [M+Na $]^{+}, 531.1349$; found: 531.1360 . $[\boldsymbol{\alpha}]_{\mathrm{D}}{ }^{20}=+7.2\left(\mathrm{c} 1.0, \mathrm{CH}_{2} \mathrm{Cl}_{2}\right)$. Chiral HPLC analysis (Chiral pak AD-H, $i$-PrOH/n-hexane $=20: 80$, flow rate $=0.5 \mathrm{~mL} / \mathrm{min}$, wave length $=254 \mathrm{~nm}), \mathrm{t}_{\mathrm{R}}($ minor enantiomer $)=58.467 \mathrm{~min}, \mathrm{t}_{\mathrm{R}}($ major enantiomer $)=$ $64.655 \mathrm{~min}, 99 \%$ ee. 

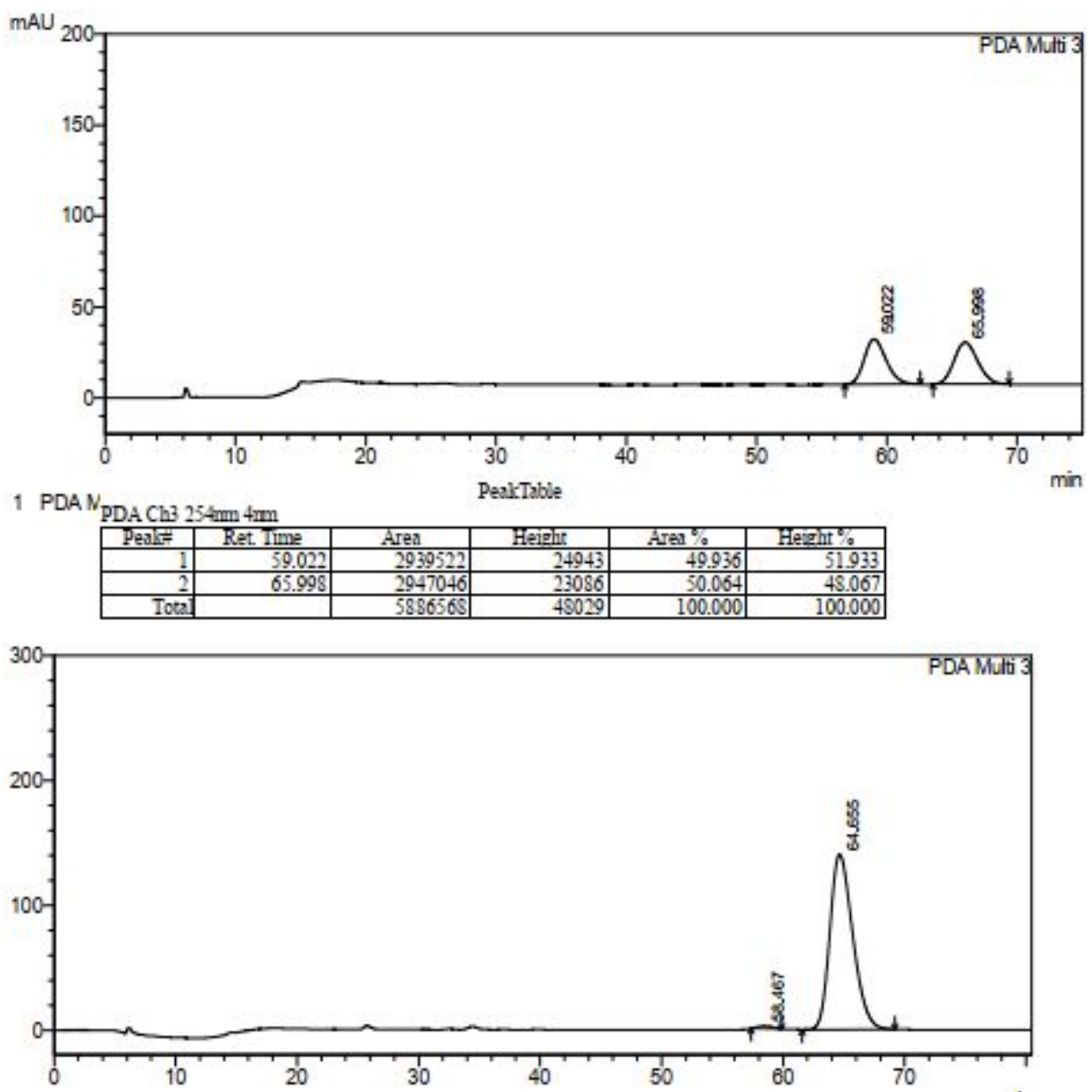

\begin{tabular}{|c|c|c|c|c|c|}
\hline PDA A & \multicolumn{5}{|c|}{ PeakThable } \\
\hline Peal= & Ret. Time & Area & Height & Aren \% & Reight \% \\
\hline & 58.467 & 75776 & 1160 & 0.411 & 0.819 \\
\hline & 64.655 & 18379648 & 140426 & 99.589 & 99.181 \\
\hline Tots & & 18455425 & 141586 & 100.000 & 100.000 \\
\hline
\end{tabular}

\section{Typical procedures and characterization data for compounds 6ab-6ai}

\subsection{General procedure for the synthesis of compounds 6ab-6ai}<smiles>O=C(NC1C(=[OH+])N(Cc2ccccc2)c2ccccc21)c1ccccc1</smiles><smiles>[R]C=CS(=O)(=O)[Ge]</smiles><smiles>[R]CC12CS(=O)(=O)N(C(=O)c3ccccc3)[C@@]1(c1ccccc1)C(=O)N2Cc1ccccc1</smiles>

A solution of 3-benzamido-2-oxindole 1a (34.2 $\mathrm{mg}, 0.1 \mathrm{mmol}), \beta$-arylethenesulfonyl fluorides $\mathbf{2 b}$-2 $\mathbf{j}$ $(0.12 \mathrm{mmol})$ and catalyst $4 \mathbf{d}(12.6 \mathrm{mg}, 0.02 \mathrm{mmol})$ in $\mathrm{CH}_{2} \mathrm{Cl}_{2}(1.0 \mathrm{~mL})$ were stirred at $\mathrm{rt}$ for $24 \mathrm{~h}$. After the solvent as evaporated under vacuum, the residue was purified by flash column chromatography over silica gel to afford the products 6ab-6ai.

5.2 General procedure for the synthesis of racemic compounds 6ab-6ai 
<smiles>[R]C=CS(=O)(=O)F</smiles>

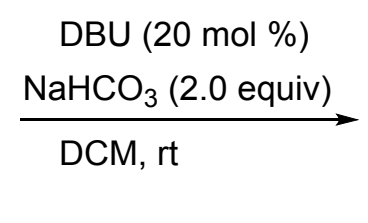<smiles>[R]CC1(C(=O)N(C(=O)c2ccccc2)c2ccccc2)C(=O)CS(=O)(=O)N1Cc1ccccc1</smiles>

A solution of 3-benzamido-2-oxindole $\mathbf{1 a}(34.2 \mathrm{mg}, 0.1 \mathrm{mmol}), \beta$-arylethenesulfonyl fluorides $\mathbf{2 b}-\mathbf{2} \mathbf{j}$ (0.12 mmol), $\mathrm{NaHCO}_{3}(16.8 \mathrm{mg}, 0.2 \mathrm{mmol})$ and $\mathrm{DBU}(3 \mathrm{mg}, 0.02 \mathrm{mmol})$ in $\mathrm{CH}_{2} \mathrm{Cl}_{2}(1.0 \mathrm{~mL})$ was stirred at $\mathrm{rt}$ for $24 \mathrm{~h}$. After the solvent was evaporated under vacuum, the residue was purified by flash column chromatography over silica gel to afford the racemic products 6ab-6ai.

\subsection{Scale-up synthesis of compound 6ab}<smiles>O=C(NC1C(=[OH+])N(Cc2ccccc2)c2ccccc21)c1ccccc1</smiles><smiles>O=C(c1ccccc1)N1C(=O)N(Cc2ccccc2)c2ccccc2[C@H]1c1ccccc1</smiles>

A solution of 3-benzamido-2-oxindole $\mathbf{1 a}$ ( $342 \mathrm{mg}, 1.5 \mathrm{mmol}$ ), $\beta$-arylethenesulfonyl fluorides $\mathbf{2 b}$ (335 $\mathrm{mg}, 1.8 \mathrm{mmol})$ and catalyst $\mathbf{4 d}(189 \mathrm{mg}, 0.3 \mathrm{mmol})$ in $\mathrm{CH}_{2} \mathrm{Cl}_{2}(15 \mathrm{~mL})$ were stirred at $\mathrm{rt}$ for $36 \mathrm{~h}$. After the solvent as evaporated under vacuum, the residue was purified by flash column chromatography over silica gel (Petroleum ether/ethyl acetate $=8: 1)$ to afford the product $\mathbf{6} \mathbf{a b}$ as a white solid $(370 \mathrm{mg}, 73 \%$ ).

\subsection{Characterizations of product 6ab-6ai}

(3S,4'S)-2'-Benzoyl-1-benzyl-4'-phenylspiro[indoline-3,3'-isothiazolidin]-2-one 1',1'-dioxide (6ab)<smiles>O=C(c1ccccc1)N1[C@]2(C(=O)Nc3ccccc32)[C@@H](c2ccccc2)CS1(=O)=O</smiles>

$6 \mathrm{ab} \stackrel{\mathrm{Bn}}{\mathrm{B}}$

Petroleum ether/ethyl acetate $=8: 1$ was used as the eluent for column chromatography. White solid (41 mg, 80\%), m. p. 249.9-251.9 ${ }^{\circ} \mathrm{C}^{1}{ }^{1} \mathbf{H}$ NMR (400 MHz, $\left.\mathrm{CDCl}_{3}\right) \delta$ 7.79-7.73 (m, 2H), 7.61-7.52 (m, 2H), 7.48-7.41 (m, 2H), 7.24-7.06 (m, 8H), 6.92-6.85 (m, 4H), $6.36(\mathrm{~d}, J=7.2 \mathrm{~Hz}, 1 \mathrm{H}), 4.80-4.70(\mathrm{~m}, 2 \mathrm{H})$, $4.49(\mathrm{dd}, J=14.8,6.0 \mathrm{~Hz}, 1 \mathrm{H}), 4.25-4.17(\mathrm{~m}, 1 \mathrm{H}), 3.87(\mathrm{dd}, J=12.8,6.0 \mathrm{~Hz}, 1 \mathrm{H}) .{ }^{13} \mathbf{C ~ N M R}(101 \mathrm{MHz}$, $\left.\mathrm{CDCl}_{3}\right) \delta 171.74,167.61,142.81,134.73,133.20,132.19,130.10,129.92,128.92,128.68,128.61$, $128.47,128.10,127.38,126.75,124.72,122.94,122.81,110.02,71.88,52.77,46.16,44.30$. HRMS (ESI)

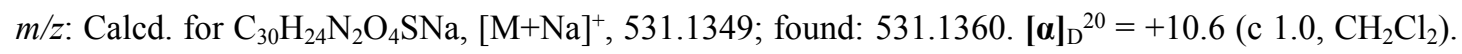
Chiral HPLC analysis (Chiral pak IG, $i-\mathrm{PrOH} / n$-hexane $=20: 80$, flow rate $=1.0 \mathrm{~mL} / \mathrm{min}$, wave length $=254 \mathrm{~nm}), \mathrm{t}_{\mathrm{R}}($ minor enantiomer $)=22.146 \mathrm{~min}, \mathrm{t}_{\mathrm{R}}($ major enantiomer $)=34.353 \mathrm{~min}, 87 \%$ ee . 


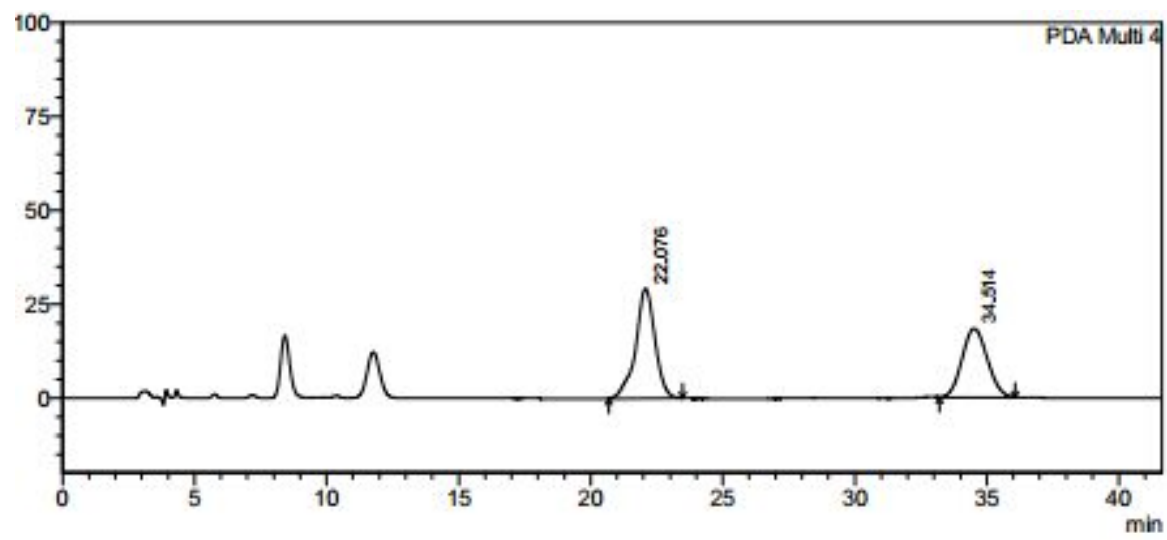

1 PDA Mutti $4 / 254 \mathrm{rm} 4 \mathrm{~nm}$
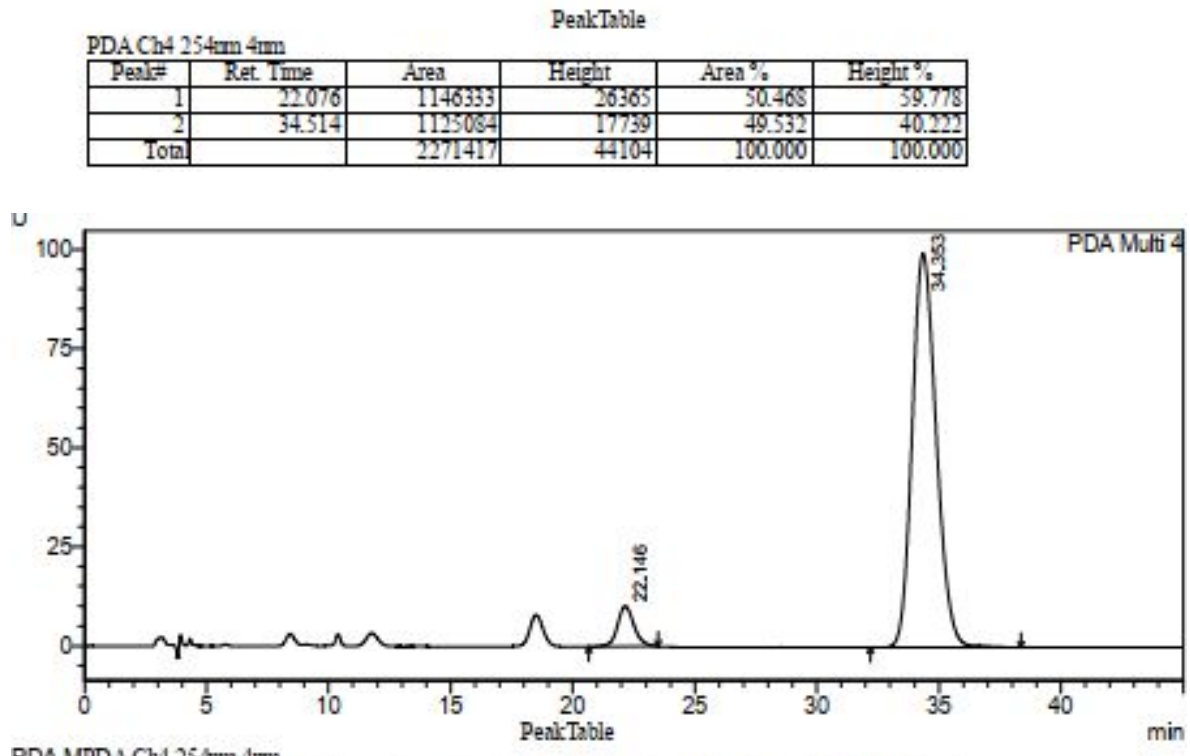

PDA MPDA Ch4 25 $4 \mathrm{~mm} 4 \mathrm{~mm}$

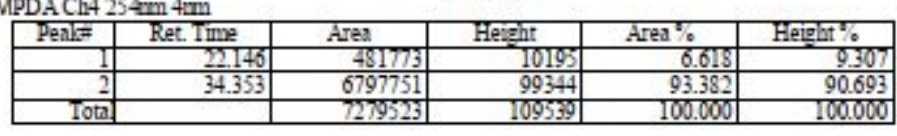

(3S,4'S)-2'-Benzoyl-1-benzyl-4'-(4-chlorophenyl)spiro[indoline-3,3'-isothiazolidin]-2-one1',1'dioxide (6ac)<smiles>CC(C)(C)OC(=O)N1C(=O)[C@@]2(c3ccccc3[C@@H](c3ccc(Cl)cc3)CS2(=O)=O)N(C(=O)c2ccccc2)S1(=O)=O</smiles>

Petroleum ether/ethyl acetate $=8: 1$ was used as the eluent for column chromatography. White solid (40 mg, 74\%), m. p. $223.5-225.3^{\circ} \mathrm{C} .{ }^{1} \mathbf{H}$ NMR $\left(400 \mathrm{MHz}, \mathrm{CDCl}_{3}\right) \delta$ 7.78-7.72 (m, $\left.2 \mathrm{H}\right), 7.60-7.51(\mathrm{~m}, 2 \mathrm{H})$, 7.47-7.41 (m, 2H), 7.25-7.15 (m, 6H), 7.11 (td, $J=7.6,0.8 \mathrm{~Hz}, 1 \mathrm{H}), 6.91-6.84(\mathrm{~m}, 2 \mathrm{H}), 6.74-6.69(\mathrm{~m}$, 2H), $6.46(\mathrm{~d}, J=7.6 \mathrm{~Hz}, 1 \mathrm{H}), 4.86(\mathrm{~d}, J=16.0 \mathrm{~Hz}, 1 \mathrm{H}), 4.67$ (d, $J=16.0 \mathrm{~Hz}, 1 \mathrm{H}), 4.43$ (dd, $J=14.4$, $5.6 \mathrm{~Hz}, 1 \mathrm{H}), 4.19-4.11(\mathrm{~m}, 1 \mathrm{H}), 3.85(\mathrm{dd}, J=12.8,6.0 \mathrm{~Hz}, 1 \mathrm{H}) .{ }^{13} \mathbf{C} \mathbf{N M R}\left(101 \mathrm{MHz}, \mathrm{CDCl}_{3}\right) \delta 171.54$, 167.58, 142.84, 134.64, 133.05, 132.30, 131.69, 130.42, 130.21, 128.96, 128.73, 128.46, 128.14, 127.59, 126.82, 124.37, 123.28, 122.98, 122.95, 110.23, 71.56, 52.66, 45.67, 44.34. HRMS (ESI) $m / z$ : Calcd. 
for $\mathrm{C}_{30} \mathrm{H}_{23} \mathrm{~N}_{2} \mathrm{O}_{4} \mathrm{SClNa}$, $[\mathrm{M}+\mathrm{Na}]^{+}, 565.0959$; found:565.0964. $[\alpha]_{\mathrm{D}}^{20}=+21.7$ (c 1.0, $\mathrm{CH}_{2} \mathrm{Cl}_{2}$ ). Chiral HPLC analysis (Chiral pak IG, $i$-PrOH $/ n$-hexane $=20: 80$, flow rate $=1.0 \mathrm{~mL} / \mathrm{min}$, wave length $=254$ $\mathrm{nm}), \mathrm{t}_{\mathrm{R}}($ minor enantiomer $)=29.4811 \mathrm{~min}, \mathrm{t}_{\mathrm{R}}($ major enantiomer $)=55.130 \mathrm{~min}, 78 \%$ ee.

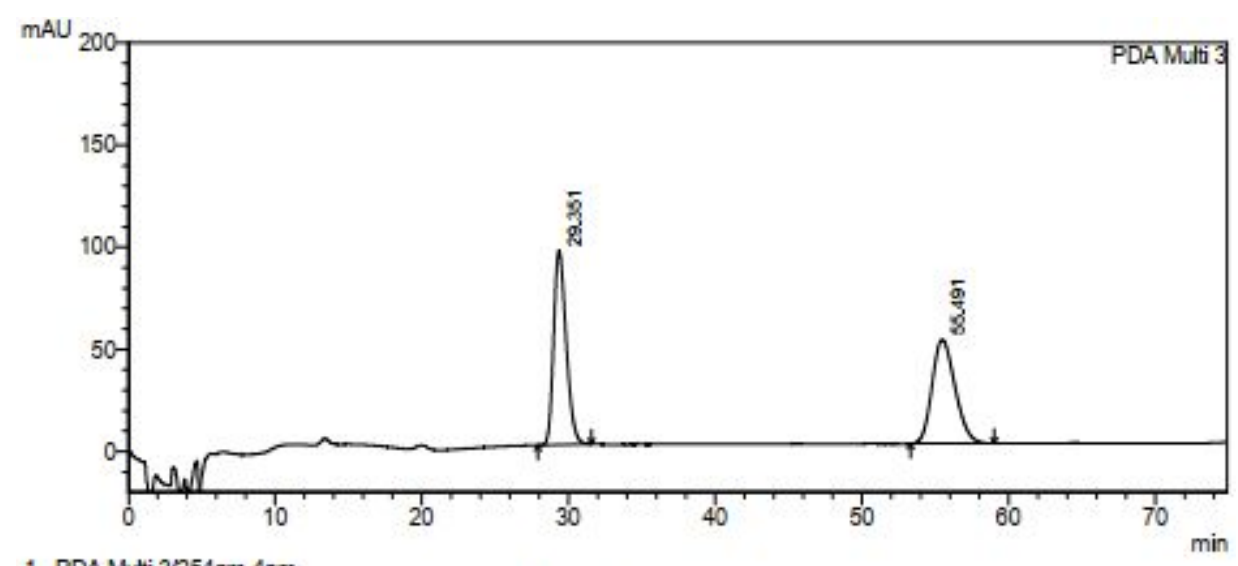

1 PDA Mutti $3 / 254 \mathrm{~mm} 4 \mathrm{~nm}$

PeakTable
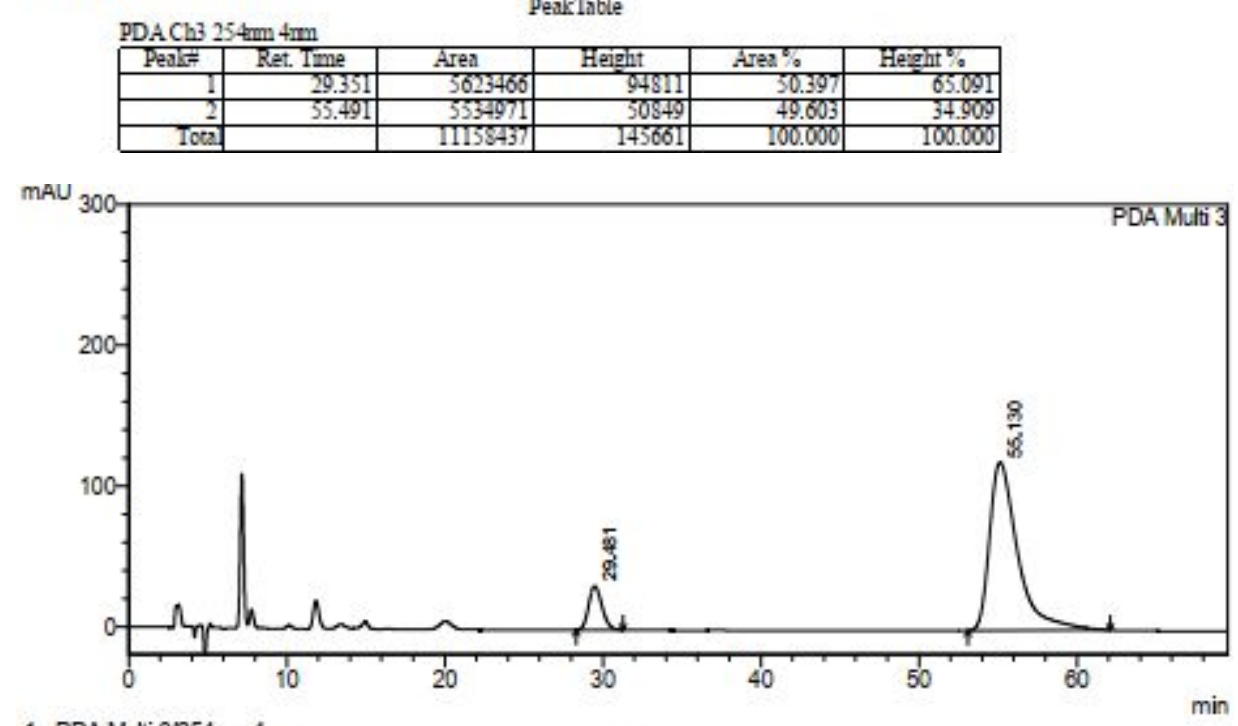

1 PDA Muti $3 / 254 \mathrm{~mm} 4 \mathrm{~nm}$

PeakTable

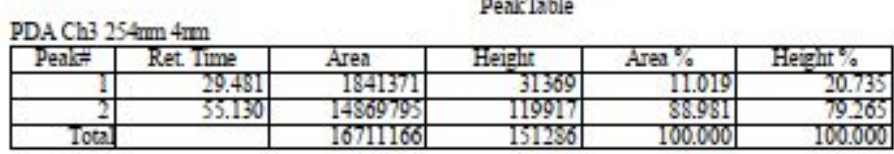

(3S,4'S)-2'-Benzoyl-1-benzyl-4'-(4-bromophenyl)spiro[indoline-3,3'-isothiazolidin]-2-one 1',1'-dioxide (6ad)<smiles>O=C(c1ccccc1)N1c2ccccc2[C@]2(C(=O)N(Cc3ccccc3)c3ccccc3)[C@H](c3ccc(Br)cc3)C[SH]12=O</smiles>

Petroleum ether/ethyl acetate $=8: 1$ was used as the eluent for column chromatography. White solid (43 mg, 73\%), m. p. $242.9-244.0^{\circ} \mathrm{C}^{1}{ }^{1} \mathbf{H}$ NMR $\left(400 \mathrm{MHz}, \mathrm{CDCl}_{3}\right) \delta$ 7.77-7.70 (m, 2H), 7.59-7.51 (m, 2H), 7.46-7.40 (m, 2H), 7.25-7.15 (m, 4H), 7.13-7.07(m, 1H), 7.06-7.00 (m, 2H), 6.90-6.84 (m, 2H), 6.80$6.74(\mathrm{~m}, 2 \mathrm{H}), 6.45(\mathrm{~d}, J=8.0 \mathrm{~Hz}, 1 \mathrm{H}), 4.84(\mathrm{~d}, J=16.0 \mathrm{~Hz}, 1 \mathrm{H}), 4.68(\mathrm{~d}, J=16.4 \mathrm{~Hz}, 1 \mathrm{H}), 4.44(\mathrm{dd}, J$ 
$=14.4,5.6 \mathrm{~Hz}, 1 \mathrm{H}), 4.19-4.09(\mathrm{~m}, 1 \mathrm{H}), 3.85(\mathrm{dd}, J=12.8,6.0 \mathrm{~Hz}, 1 \mathrm{H}) .{ }^{13} \mathbf{C} \mathbf{N M R}\left(101 \mathrm{MHz}, \mathrm{CDCl}_{3}\right) \delta$ $171.55,167.59,142.83,135.08,134.65,133.07,132.30,130.40,129.93,128.73,128.70,128.46,128.14$, 127.59, 126.82, 124.40, 122.98, 122.95, 110.21, 71.64, 52.71, 45.60, 44.32. HRMS (ESI) m/z: Calcd.

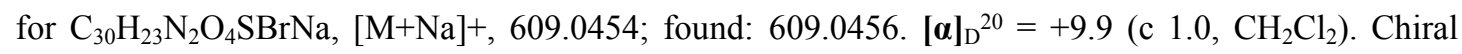
HPLC analysis (Chiral pak IG, $i$-PrOH $/ n$-hexane $=20: 80$, flow rate $=1.0 \mathrm{~mL} / \mathrm{min}$, wave length $=254$ $\mathrm{nm}), \mathrm{t}_{\mathrm{R}}($ minor enantiomer $)=31.598 \mathrm{~min}, \mathrm{t}_{\mathrm{R}}($ major enantiomer $)=58.984 \mathrm{~min}, 82 \%$ ee.

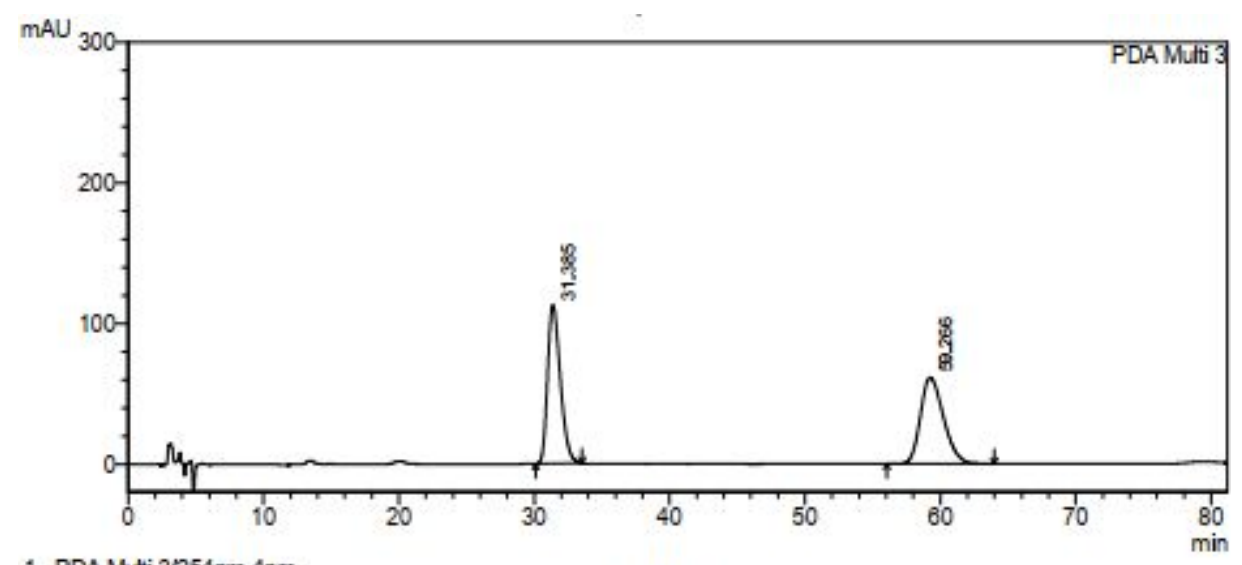

1 PDA Muti $3 / 254 \mathrm{~mm} 4 \mathrm{~nm}$
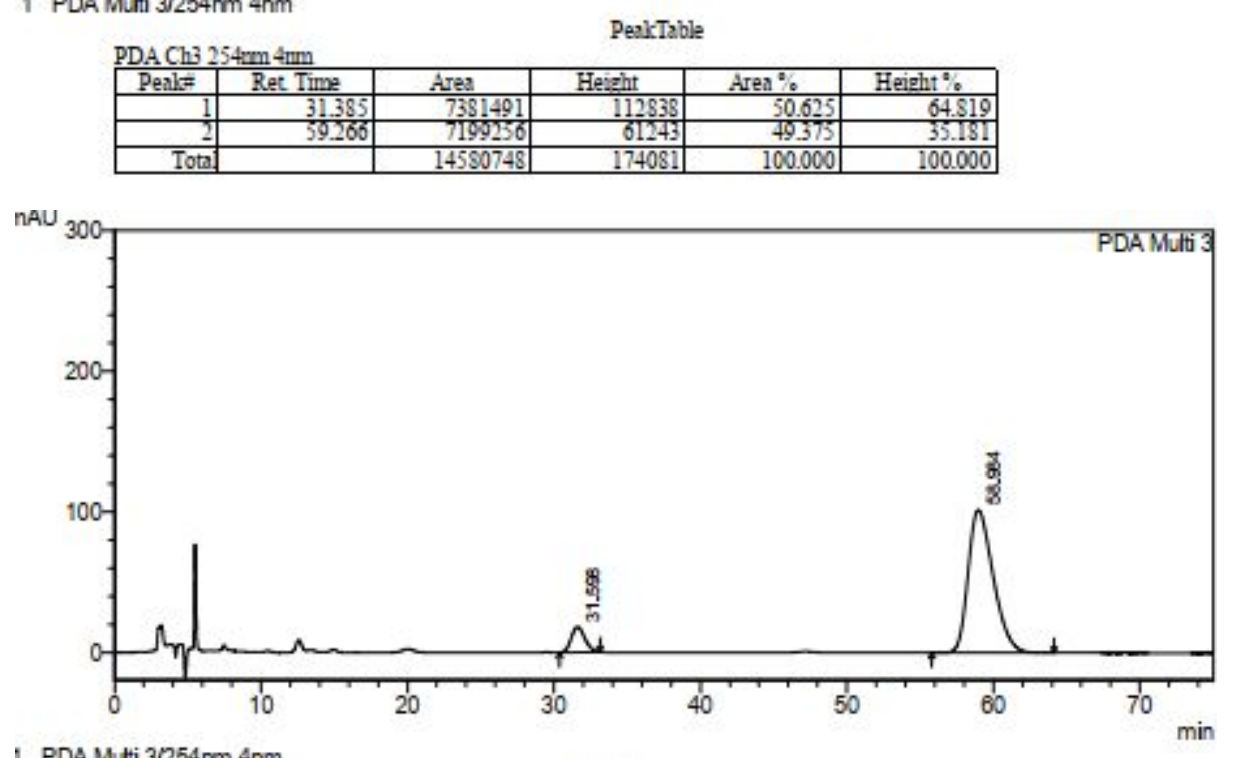

I PDA Muti $3 / 254 \mathrm{~mm} 4 \mathrm{~nm}$

PeakTable

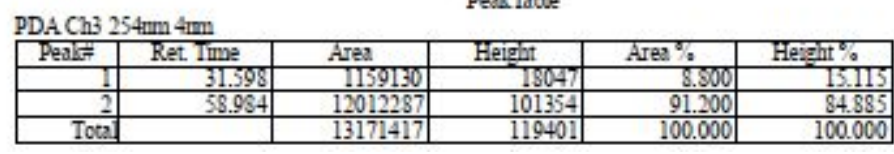

(3S,4'S)-2'-Benzoyl-1-benzyl-4'-(3-bromophenyl)spiro[indoline-3,3'-isothiazolidin]-2-one 1',1'dioxide (6ae)<smiles>O=C(Oc1ccccc1)N1C(=O)[C@@]2(c3ccccc3N1C(=O)c1ccccc1)[C@@H](c1cccc(Br)c1)CS(=O)(=O)N2Cc1ccccc1</smiles>

Petroleum ether/ethyl acetate $=8: 1$ was used as the eluent for column chromatography. White solid (46 
mg, 78\%), m. p. $255.7-256.8^{\circ} \mathrm{C}^{1}{ }^{1} \mathbf{H}$ NMR $\left(400 \mathrm{MHz}, \mathrm{CDCl}_{3}\right) \delta$ 7.80-7.72 (m, 2H), 7.59-7.51 (m, 2H), 7.48-7.41 (m, 2H), $7.35(\mathrm{dd}, J=8.0,0.8 \mathrm{~Hz}, 1 \mathrm{H}), 7.24-7.08(\mathrm{~m}, 5 \mathrm{H}), 7.05(\mathrm{t}, J=1.6 \mathrm{~Hz}, 1 \mathrm{H}), 6.99-6.89$ $(\mathrm{m}, 3 \mathrm{H}), 6.78(\mathrm{~d}, J=8.0 \mathrm{~Hz}, 1 \mathrm{H}), 6.42(\mathrm{~d}, J=7.6 \mathrm{~Hz}, 1 \mathrm{H}), 4.85-4.70(\mathrm{~m}, 2 \mathrm{H}), 4.43$ (dd, $J=14.4,6.0 \mathrm{~Hz}$, 1H), 4.21-4.10 (m, 1H), 3.86 (dd, $J=12.8,6.0 \mathrm{~Hz}, 1 \mathrm{H}) .{ }^{13} \mathbf{C}$ NMR (101 MHz, $\left.\mathrm{CDCl}_{3}\right) \delta 171.57,167.55$, 142.71, 134.65, 133.07, 132.29, 132.10, 131.71, 130.40, 129.87, 128.81, 128.46, 128.15, 127.53, 127.15, 126.74, 124.34, 123.00, 122.89, 122.45, 110.24, 71.65, 52.54, 45.66, 44.40. HRMS (ESI) m/z: Calcd.

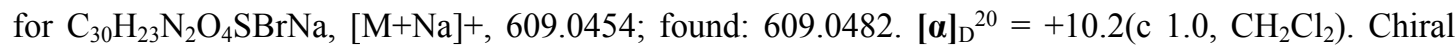
HPLC analysis (Chiral pak IG, $i$-PrOH $/ n$-hexane $=20: 80$, flow rate $=1.0 \mathrm{~mL} / \mathrm{min}$, wave length $=254$ $\mathrm{nm}), \mathrm{t}_{\mathrm{R}}($ minor enantiomer $)=18.496 \mathrm{~min}, \mathrm{t}_{\mathrm{R}}($ major enantiomer $)=48.321 \mathrm{~min}, 95 \%$ ee.

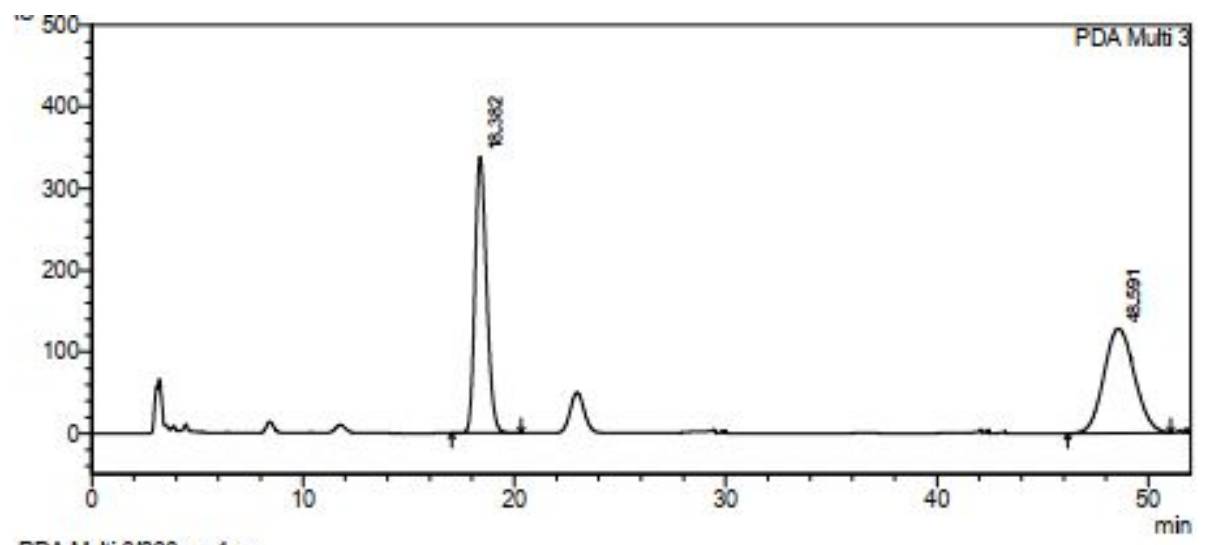

PDA Muti $3 / 230 \mathrm{~mm} 4 \mathrm{~nm}$
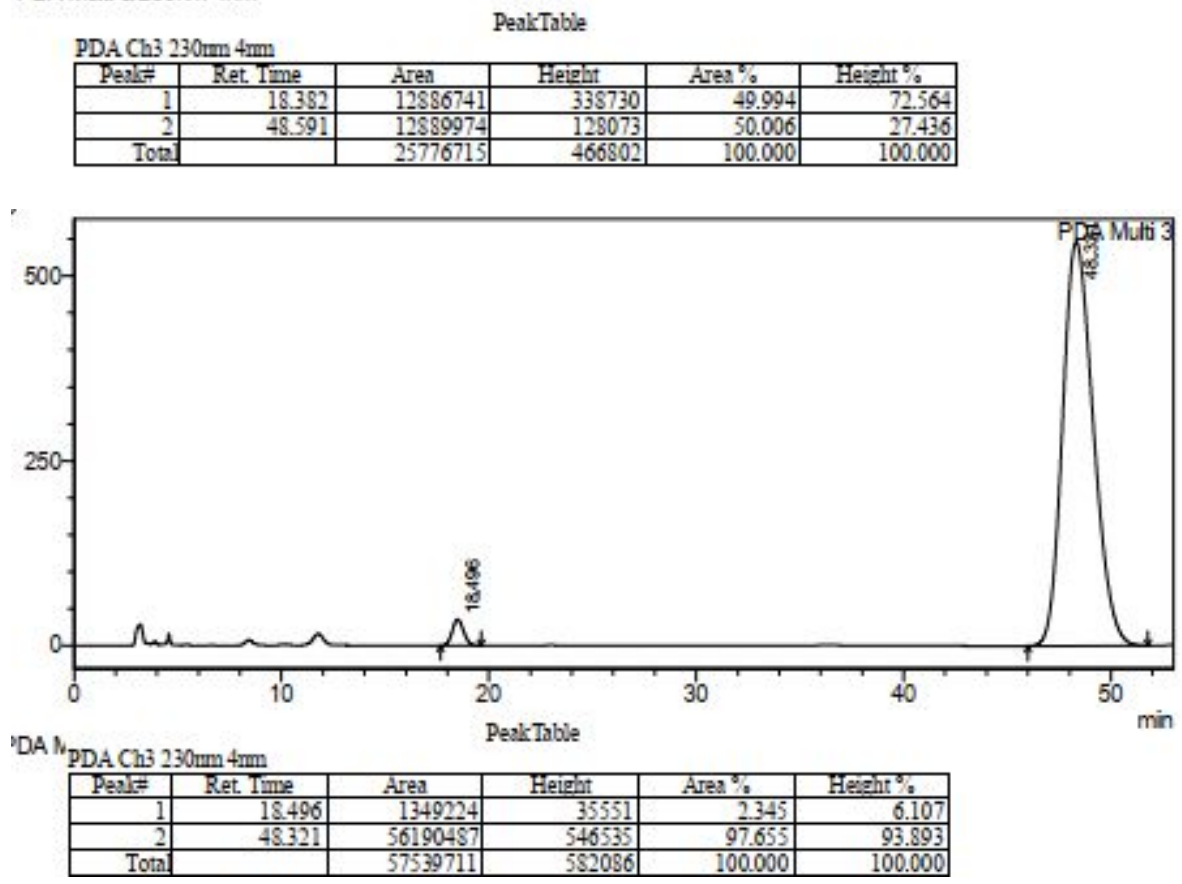

(3S,4'S)-2'-Benzoyl-1-benzyl-4'-(3-methoxyphenyl)spiro[indoline-3,3'-isothiazolidin]-2-one $1^{\prime}, 1^{\prime}$ dioxide (6af) 
<smiles>COc1cccc([C@@H]2CS(=O)(=O)N(C(=O)c3ccccc3)[C@]23C(=O)N(Cc2ccccc2)c2ccccc23)c1</smiles>

Petroleum ether/ethyl acetate $=8: 1$ was used as the eluent for column chromatography. White solid (37 mg, 69\%), m. p. $233.4-234.8^{\circ} \mathrm{C} .{ }^{1} \mathbf{H}$ NMR $\left(400 \mathrm{MHz}, \mathrm{CDCl}_{3}\right) \delta$ 7.94-7.88 (m, 2H), 7.61-7.55 (m, 2H), 7.50-7.44 (m, 2H), 7.19-7.08 (m, 6H), $6.86(\mathrm{dd}, J=8.0,2.0 \mathrm{~Hz}, 1 \mathrm{H}), 6.66(\mathrm{~d}, J=6.4 \mathrm{~Hz}, 2 \mathrm{H}), 6.53(\mathrm{~d}, J$ $=7.6 \mathrm{~Hz}, 1 \mathrm{H}), 6.38(\mathrm{~d}, J=6.8 \mathrm{~Hz}, 1 \mathrm{H}), 6.29-6.24(\mathrm{~m}, 1 \mathrm{H}), 4.85(\mathrm{~d}, J=16.4 \mathrm{~Hz}, 1 \mathrm{H}), 4.80-4.71(\mathrm{~m}, 1 \mathrm{H})$, $4.47(\mathrm{~d}, J=16.0 \mathrm{~Hz}, 1 \mathrm{H}), 4.36$ (dd, $J=14.4,4.8 \mathrm{~Hz}, 1 \mathrm{H}), 3.59$ (dd, $J=11.6,4.8 \mathrm{~Hz}, 1 \mathrm{H}), 3.48(\mathrm{~s}, 3 \mathrm{H})$. ${ }^{13}$ C NMR (101 MHz, $\left.\mathrm{CDCl}_{3}\right) \delta 172.61,167.97,159.52,144.09,134.97,133.63,133.07,131.41,130.38$, 129.74, 128.87, 128.62, 128.43, 127.26, 126.49, 125.62, 123.27, 122.07, 120.32, 115.42, 113.48, 109.90, 70.95, 55.10, 50.02, 45.87, 44.25. HRMS (ESI) $m / z$ : Calcd. for $\mathrm{C}_{31} \mathrm{H}_{26} \mathrm{~N}_{2} \mathrm{O}_{5} \mathrm{SNa}$, [M+Na] $]^{+}, 561.1455$; found: 561.1465 . $[\alpha]_{\mathrm{D}}^{20}=+18.1\left(\mathrm{c} 1.0, \mathrm{CH}_{2} \mathrm{Cl}_{2}\right.$ ). Chiral HPLC analysis (Chiral pak IG, $i-\mathrm{PrOH} / n$-hexane $=20: 80$, flow rate $=1.0 \mathrm{~mL} / \mathrm{min}$, wave length $=254 \mathrm{~nm}), \mathrm{t}_{\mathrm{R}}($ minor enantiomer $)=47.479 \mathrm{~min}, \mathrm{t}_{\mathrm{R}}($ major enantiomer) $=81.031 \mathrm{~min}, 87 \%$ ee.

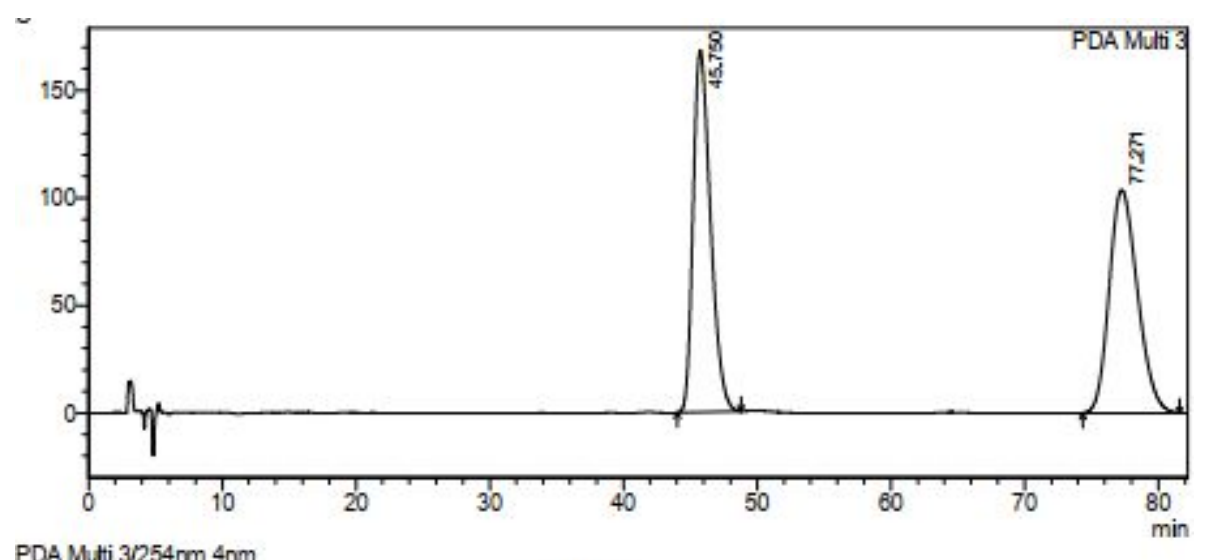

PDA Mutti $3 / 254 \mathrm{~nm} 4 \mathrm{~nm}$

PeakTable

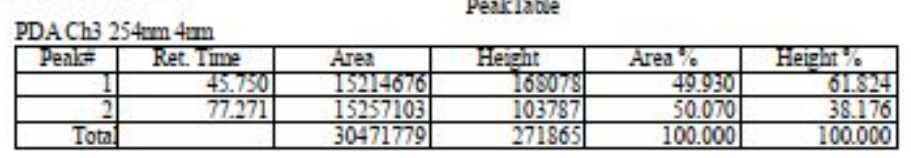




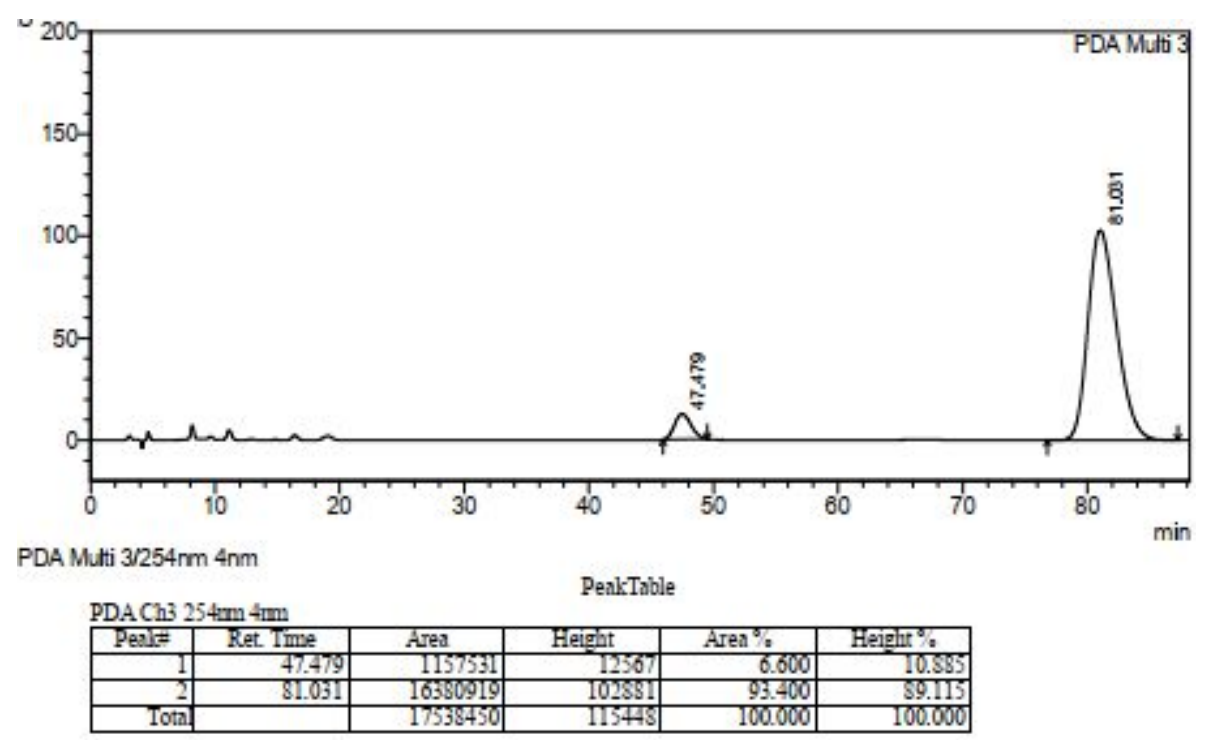

(3S,4'S)-2'-Benzoyl-1-benzyl-4'-(3,5-bis(trifluoromethyl)phenyl)spiro[indoline-3,3'isothiazolidin]-2-one 1',1'-dioxide (6ag)<smiles>O=C(c1ccccc1)N1c2ccccc2[C@]2(C(=O)N(Cc3ccccc3)c3ccccc3)[C@H](c3cc(C(F)(F)F)cc(C(F)(F)F)c3)CS(=O)(=O)N12</smiles>

Petroleum ether/ethyl acetate $=8: 1$ was used as the eluent for column chromatography. White solid (40 mg, 62\%), m. p. $228.8-230.1^{\circ} \mathrm{C} .{ }^{1} \mathbf{H}$ NMR $\left(500 \mathrm{MHz}, \mathrm{CDCl}_{3}\right) \delta 7.91(\mathrm{~d}, J=7.5 \mathrm{~Hz}, 2 \mathrm{H}), 7.75(\mathrm{~s}, 1 \mathrm{H})$, 7.64-7.57 (m, 2H), 7.49 (t, $J=7.5 \mathrm{~Hz}, 2 \mathrm{H}), 7.29-7.24(\mathrm{~m}, 2 \mathrm{H}), 7.22-7.14(\mathrm{~m}, 6 \mathrm{H}), 6.81(\mathrm{~d}, J=7.5 \mathrm{~Hz}$, $2 \mathrm{H}), 6.52(\mathrm{~d}, J=7.5 \mathrm{~Hz}, 1 \mathrm{H}), 4.81(\mathrm{t}, J=13.0 \mathrm{~Hz}, 1 \mathrm{H}), 4.57-4.50(\mathrm{~m}, 2 \mathrm{H}), 4.46(\mathrm{dd}, J=14.5,4.0 \mathrm{~Hz}$, $1 \mathrm{H}), 3.65(\mathrm{dd}, J=11.0,4.0 \mathrm{~Hz}, 1 \mathrm{H}) .{ }^{13} \mathbf{C}$ NMR $\left(126 \mathrm{MHz}, \mathrm{CDCl}_{3}\right) \delta 171.86,167.73,143.57,134.64$, 133.31, 133.28, $131.88\left(\mathrm{q}, J_{\mathrm{C}-\mathrm{F}}=33.7 \mathrm{~Hz}\right), 128.87,128.66,128.52,128.38,127.60,126.76,124.35$, 123.87, 123.68, 122.92, 122.18, 121.51, 110.07, 70.38, 49.23, 45.48, 44.16. HRMS (ESI) $\mathrm{m} / z$ : Calcd. for $\mathrm{C}_{32} \mathrm{H}_{22} \mathrm{~N}_{2} \mathrm{O}_{4} \mathrm{~F}_{6} \mathrm{SNa}$, [M+Na] $]^{+}$, 667.1097; found: 667.1085. $[\alpha]_{\mathrm{D}^{20}}=+12.0\left(\mathrm{c}\right.$ 1.0, $\mathrm{CH}_{2} \mathrm{Cl}_{2}$ ). Chiral HPLC analysis (Chiral pak IG, $i-\mathrm{PrOH} / n$-hexane $=20: 80$, flow rate $=1.0 \mathrm{~mL} / \mathrm{min}$, wave length $=254$ $\mathrm{nm}), \mathrm{t}_{\mathrm{R}}($ minor enantiomer $)=5.308 \mathrm{~min}, \mathrm{t}_{\mathrm{R}}($ major enantiomer $)=8.419 \mathrm{~min}, 71 \%$ ee. 


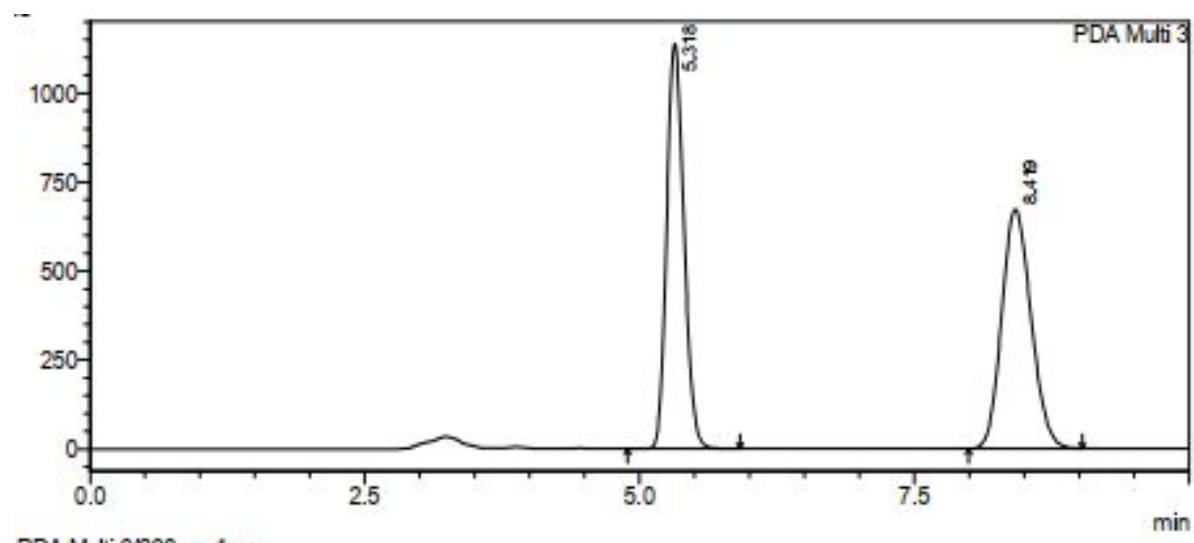

PDA Mutti 3/230 $\mathrm{mm} 4 \mathrm{~nm}$

PeakTable
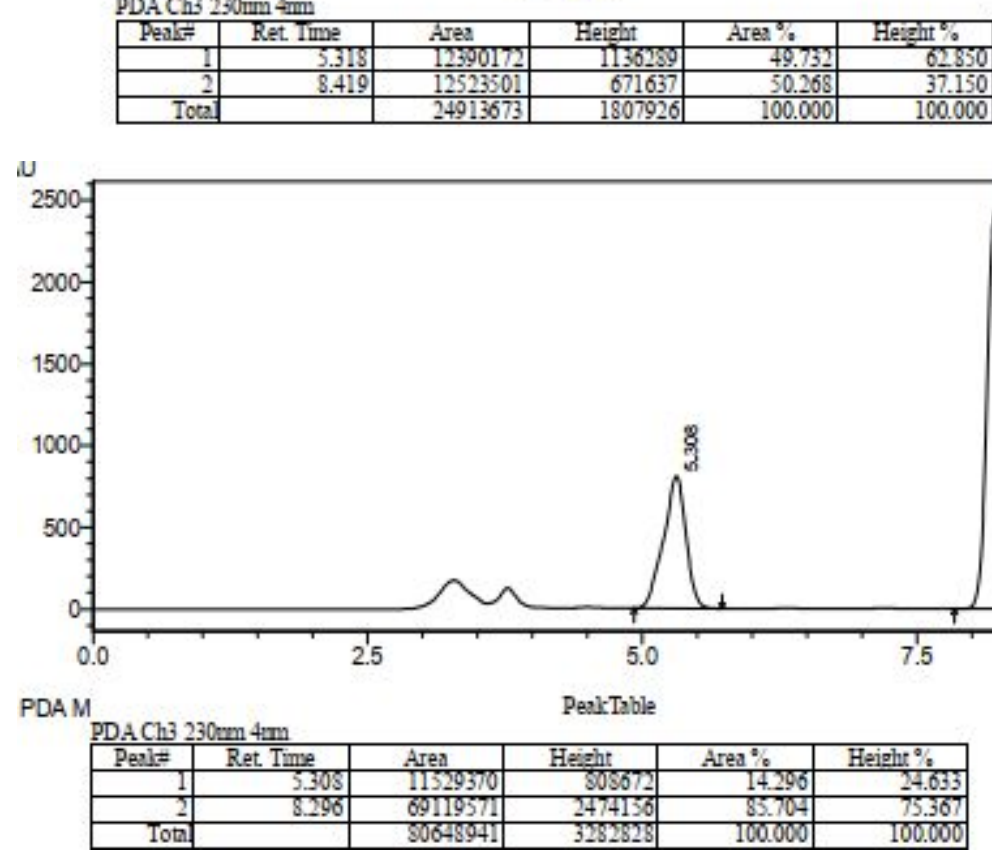

(3S,4'S)-2'-Benzoyl-1-benzyl-4'-(thiophen-3-yl)spiro[indoline-3,3'-isothiazolidin]-2-one1',1'dioxide (6ah)<smiles>O=C(c1ccccc1)N1C(=O)[C@]2(c3ccccc31)[C@H](c1ccsc1)C(=O)N2Cc1ccccc1</smiles>

Petroleum ether/ethyl acetate $=6: 1$ was used as the eluent for column chromatography. White solid (31 mg, 60\%), m. p. $209.5-211.4^{\circ} \mathrm{C} .{ }^{1} \mathbf{H}$ NMR $\left(400 \mathrm{MHz}, \mathrm{CDCl}_{3}\right) \delta$ 7.88-7.71 (m, 2H), 7.61-7.53 (m, 2H), 7.47 (t, $J=7.6 \mathrm{~Hz}, 2 \mathrm{H}), 7.30-7.19$ (m, 5H), 7.03-6.97 (m, 2H), 7.03-6.97 (m, 2H), 6.91 (dd, $J=2.8,0.8$ $\mathrm{Hz}, 1 \mathrm{H}), 6.50(\mathrm{~d}, J=7.6 \mathrm{~Hz}, 1 \mathrm{H}), 6.47(\mathrm{dd}, J=5.2,1.2 \mathrm{~Hz}, 1 \mathrm{H}), 4.88(\mathrm{~d}, J=16.0 \mathrm{~Hz}, 1 \mathrm{H}), 4.79(\mathrm{~d}, J=$ $16.0 \mathrm{~Hz}, 1 \mathrm{H}), 4.62(\mathrm{dd}, J=14.0,6.0 \mathrm{~Hz}, 1 \mathrm{H}), 4.17-4.06(\mathrm{~m}, 1 \mathrm{H}), 3.93(\mathrm{dd}, J=12.8,6.4 \mathrm{~Hz}, 1 \mathrm{H}){ }^{13} \mathbf{C}$ NMR $\left(101 \mathrm{MHz}, \mathrm{CDCl}_{3}\right) \delta 171.88,167.70,142.94,134.81,133.12,132.28,130.90,130.25,128.78$, $128.52,128.12,127.48,126.78,126.67,126.14,125.14,125.05,122.90,122.83,110.09,71.42,53.47$, 44.36, 41.96. HRMS (ESI) $m / z$ : Calcd. for $\mathrm{C}_{28} \mathrm{H}_{22} \mathrm{~N}_{2} \mathrm{O}_{4} \mathrm{~S}_{2} \mathrm{Na}$, [M+Na] ${ }^{+}$, 537.0913; found: 537.0908. 
$[\boldsymbol{\alpha}]_{\mathrm{D}}{ }^{20}=+19.2\left(\mathrm{c} 1.0, \mathrm{CH}_{2} \mathrm{Cl}_{2}\right)$. Chiral HPLC analysis (Chiral pak IG, $i$-PrOH/n-hexane $=20: 80$, flow rate $=1.0 \mathrm{~mL} / \mathrm{min}$, wave length $=254 \mathrm{~nm}), \mathrm{t}_{\mathrm{R}}($ minor enantiomer $)=45.420 \mathrm{~min}, \mathrm{t}_{\mathrm{R}}($ major enantiomer $)=$ $69.740 \mathrm{~min}, 91 \%$ ee.

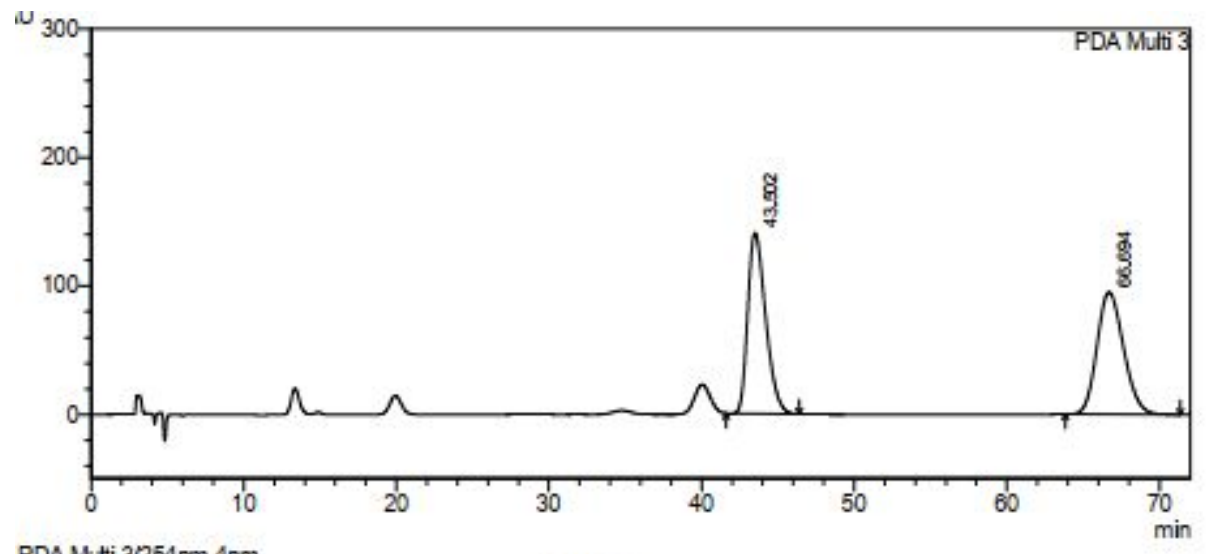

PDA Muti $3 / 254 \mathrm{rm} 4 \mathrm{~nm}$

PeakTable
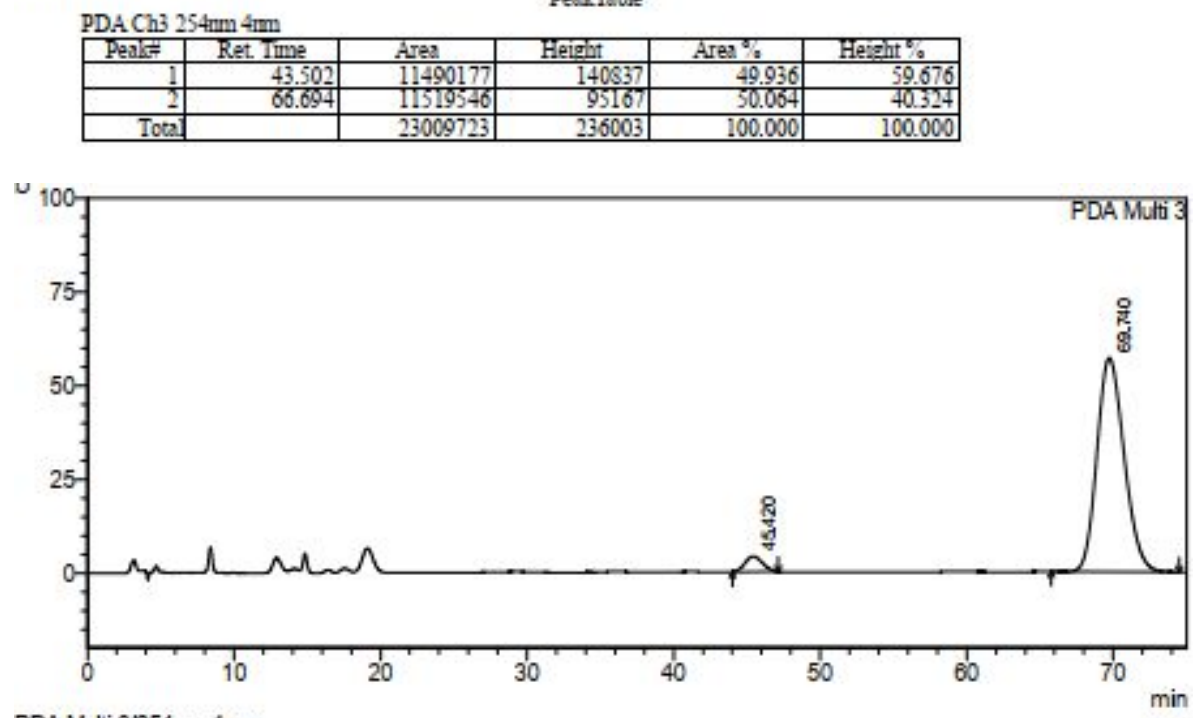

PDA Muti $3 / 254 \mathrm{rm} 4 \mathrm{~nm}$

PeskTable

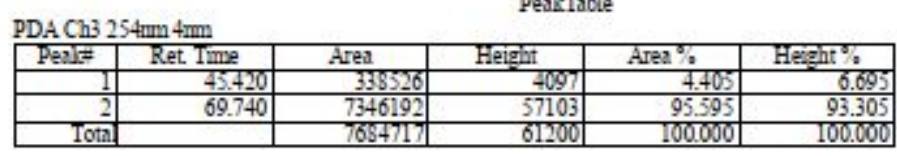

(3S,4'S)-2'-Benzoyl-1-benzyl-4' -(thiophen-2-yl)spiro[indoline-3,3'-isothiazolidin]-2-one dioxide (6ai)<smiles>O=C(c1ccccc1)N1[C@]2(C(=O)Nc3ccccc32)[C@@H](c2cccs2)CS1(=O)=O</smiles>

6ai $\mathrm{Bn}$

Petroleum ether/ethyl acetate $=6: 1$ was used as the eluent for column chromatography. White solid (29 mg, 57\%), m. p. $211.5-213.5^{\circ} \mathrm{C}^{1}{ }^{1} \mathbf{H}$ NMR $\left(400 \mathrm{MHz}, \mathrm{CDCl}_{3}\right) \delta$ 7.98-7.85 (m, 2H), 7.62-7.51 (m, 2H), 7.50-7.44 (m, 2H), 7.24-7.18 (m, 4H), 7.17-7.10 (m, 2H), $6.90(\mathrm{dd}, J=5.2,3.6 \mathrm{~Hz}, 1 \mathrm{H}), 6.85-6.78(\mathrm{~m}$, 2H), $6.74(\mathrm{~d}, J=3.6 \mathrm{~Hz}, 1 \mathrm{H}), 6.48(\mathrm{~d}, J=8.0 \mathrm{~Hz}, 1 \mathrm{H}), 4.89$ (d, $J=16.0 \mathrm{~Hz}, 1 \mathrm{H}), 4.74(\mathrm{dd}, J=14.4,11.6$ $\mathrm{Hz}, 1 \mathrm{H}), 4.64-4.52$ (m, 2H), 3.69 (dd, $J=11.6,4.8 \mathrm{~Hz}, 1 \mathrm{H}) .{ }^{13} \mathbf{C}$ NMR $\left(101 \mathrm{MHz}, \mathrm{CDCl}_{3}\right) \delta 172.57$, 
$168.00,144.42,135.05,133.55,133.12,131.90,130.64,128.93,128.65,128.42,127.31,127.12,127.03$, 126.77, 126.23, 125.25, 123.38, 122.14, 109.88, 70.74, 50.88, 44.33, 42.01. HRMS (ESI) m/z: Calcd.

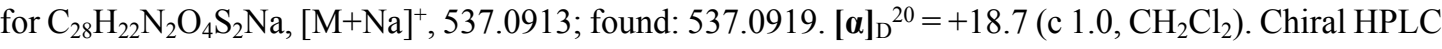
analysis (Chiral pak IG, $i$-PrOH $/ n$-hexane $=20: 80$, flow rate $=1.0 \mathrm{~mL} / \mathrm{min}$, wave length $=254 \mathrm{~nm}$ ), $\mathrm{t}_{\mathrm{R}}$ $($ minor enantiomer $)=41.459 \mathrm{~min}, \mathrm{t}_{\mathrm{R}}($ major enantiomer $)=61.873 \mathrm{~min}, 91 \% \mathrm{ee}$.

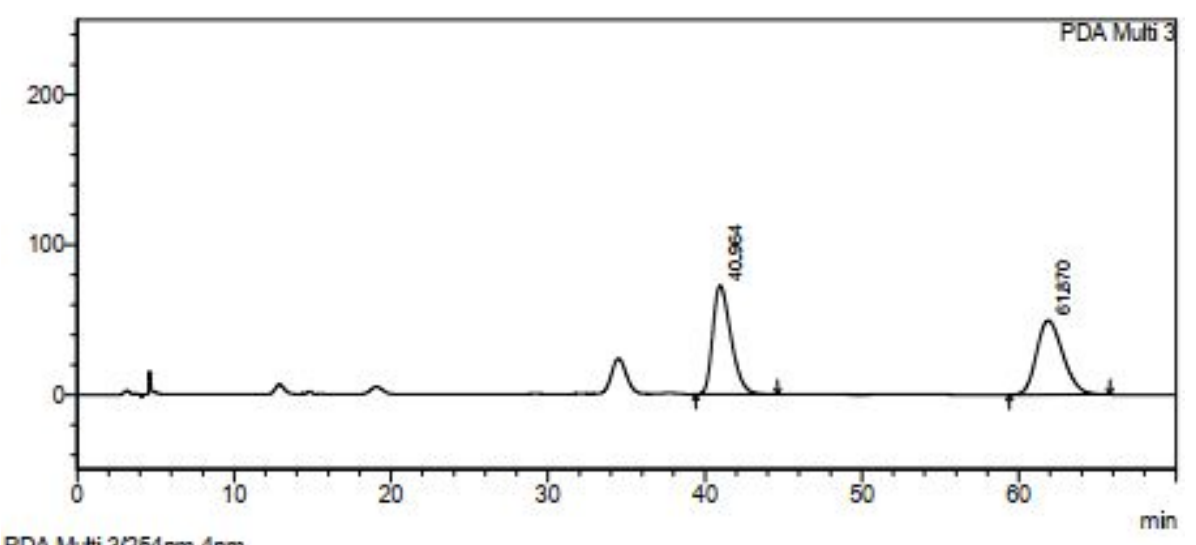

PDA Mutti $3 / 254 \mathrm{~nm} 4 \mathrm{~nm}$
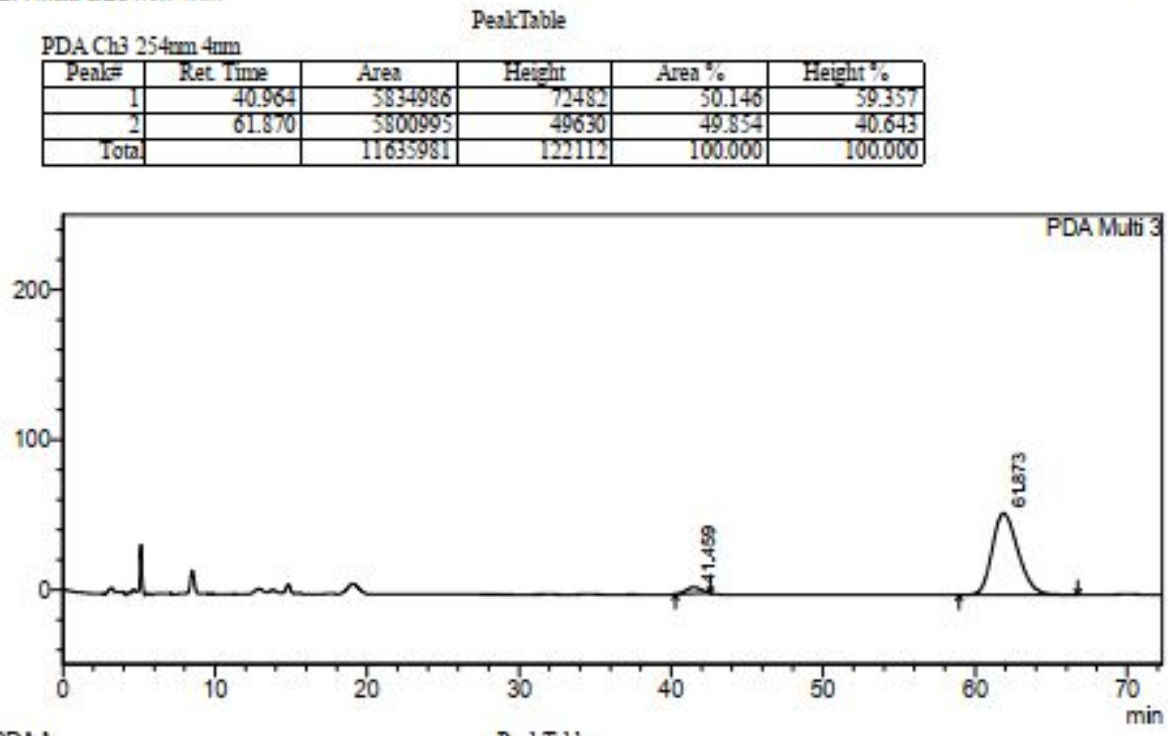

PDA N

PenkTable

\begin{tabular}{|c|c|c|c|c|c|}
\hline \multicolumn{6}{|c|}{ PDACh3 254m $4 \mathrm{~mm}$} \\
\hline Peali: & Ret. Trme & Area & Heigit & Area \% & Height\% \\
\hline 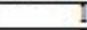 & 41.459 & 295758 & 4298 & 4.419 & 7.321 \\
\hline 2 & 61.873 & 6397758 & 54405 & 95.581 & 92.679 \\
\hline$T_{002}$ & & 6693517 & 58703 & 100.000 & 00000 \\
\hline
\end{tabular}

\section{Procedure for the gram-scale synthesis of 3a}<smiles>O=C(NC1C(=O)N(Cc2ccccc2)c2ccccc21)c1ccccc1</smiles>

1a<smiles>C=C[C@H](OC)[C@H](C)OS(=O)(=O)F</smiles>

$2 a$<smiles>O=C(N[C@]1(CCS(=O)(=O)F)C(=O)N(Cc2ccccc2)c2ccccc21)Pc1ccccc1</smiles>

3a $1.3 \mathrm{~g}$
$(3.0 \mathrm{mmol})$
$(3.3 \mathrm{mmol})$
$96 \%$ yield, $99 \%$ ee

A solution of 3-benzamido-2-oxindole 1a (1.03 g, $3 \mathrm{mmol}$ ), ethylene sulfonyl fluoride $2 \mathbf{a}$ (396 mg, 3.6 $\mathrm{mmol}$ ) and catalyst $\mathbf{4 d}(378 \mathrm{mg}, 0.6 \mathrm{mmol})$ in $\mathrm{CH}_{2} \mathrm{Cl}_{2}(10 \mathrm{~mL})$ was stirred at $\mathrm{rt}$ for $24 \mathrm{~h}$. After the solvent 
was evaporated under vacuum, the residue was purified by flash column chromatography over silica gel (Petroleum ether/ethyl acetate $=8: 1)$ to afford the product $\mathbf{3 a}$ as a white solid $(1.3 \mathrm{~g}, 96 \%)$.

\section{Procedures for the transformations of $3 a$}

Synthesis of (S)- $N$-(1-enzyl-2-oxo-3-(2-(pyrrolidin-1-ylsulfonyl)ethyl)indolin-3-yl)benzamide (7a)<smiles>O=C([PH2+])N[C@@H](C(=O)N[C@@]1(CCS(=O)(=O)F)C(=O)N(Cc2ccccc2)c2ccccc21)c1ccccc1</smiles>

$3 a$

7 a, $70 \%$ yield, $99 \%$ ee

A solution of (S)-2-(3-benzamido-1-benzyl-2-oxoindolin-3-yl)ethane-1-sulfonyl fluoride 3a (45.2 mg, $0.1 \mathrm{mmol})$, pyrrolidine $(10.7 \mathrm{mg}, 0.15 \mathrm{mmol})$ in THF $(10 \mathrm{~mL})$ was stirred in an oil bath at $60{ }^{\circ} \mathrm{C}$ until the completion of the reaction ( $12 \mathrm{~h})$ as indicated by TLC. After the solvent was evaporated under vacuum, the residue was purified by flash column chromatography over silica gel (Petroleum ether/ethyl acetate $=8: 1)$ to afford the product $7 \mathbf{a}$ as a white solid $(35.2 \mathrm{mg}, 70 \%)$. m. p. $166.5-167.4{ }^{\circ} \mathrm{C} .{ }^{1} \mathbf{H}$ NMR $(400$ $\left.\mathrm{MHz} \mathrm{CDCl}_{3}\right) \delta 8.31(\mathrm{~s}, 1 \mathrm{H}), 7.92(\mathrm{~d}, J=7.2 \mathrm{~Hz}, 2 \mathrm{H}), 7.52-7.46(\mathrm{~m}, 1 \mathrm{H}), 7.46-7.39(\mathrm{~m}, 4 \mathrm{H}), 7.35(\mathrm{t}, J=$ $7.6 \mathrm{~Hz}, 2 \mathrm{H}), 7.28$ (d, $J=7.2 \mathrm{~Hz}, 2 \mathrm{H}), 7.19$ (t, $J=7.6 \mathrm{~Hz}, 1 \mathrm{H}), 7.02(\mathrm{t}, J=7.6 \mathrm{~Hz}, 1 \mathrm{H}), 6.74(\mathrm{~d}, J=7.6$ $\mathrm{Hz}, 1 \mathrm{H}), 5.08$ (d, $J=16.0 \mathrm{~Hz}, 1 \mathrm{H}), 4.95$ (d, $J=16.0 \mathrm{~Hz}, 1 \mathrm{H}), 3.82-3.72(\mathrm{~m}, 1 \mathrm{H}), 3.42-3.29(\mathrm{~m}, 4 \mathrm{H})$, 3.14-3.04 (m, 1H), 2.73-2.64 (m, 1H), 2.32-2.23 (m, 1H), 1.97-1.87 (m, 4H). ${ }^{13} \mathrm{C}$ NMR (101 MHz, $\left.\mathrm{CDCl}_{3}\right) \delta 175.95,166.26,142.21,135.86,132.59,131.93,129.95,129.18,128.88,128.48,127.61$, 127.56, 127.25, 123.12, 122.25, 109.58, 59.62, 47.97, 44.24, 42.07, 30.14, 25.85. HRMS (ESI) $\mathrm{m} / \mathrm{z}$ :

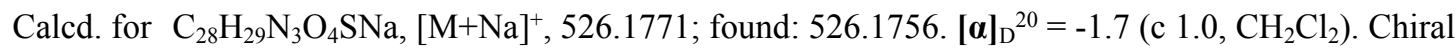
HPLC analysis (Chiral pak AD-H, $i$-PrOH $/ n$-hexane $=20: 80$, flow rate $=0.5 \mathrm{~mL} / \mathrm{min}$, wave length $=$ $254 \mathrm{~nm}), t_{R}($ major enantiomer $)=29.630 \mathrm{~min}, \mathrm{t}_{\mathrm{R}}($ minor enantiomer $)=39.118 \mathrm{~min}, 99 \%$ ee.

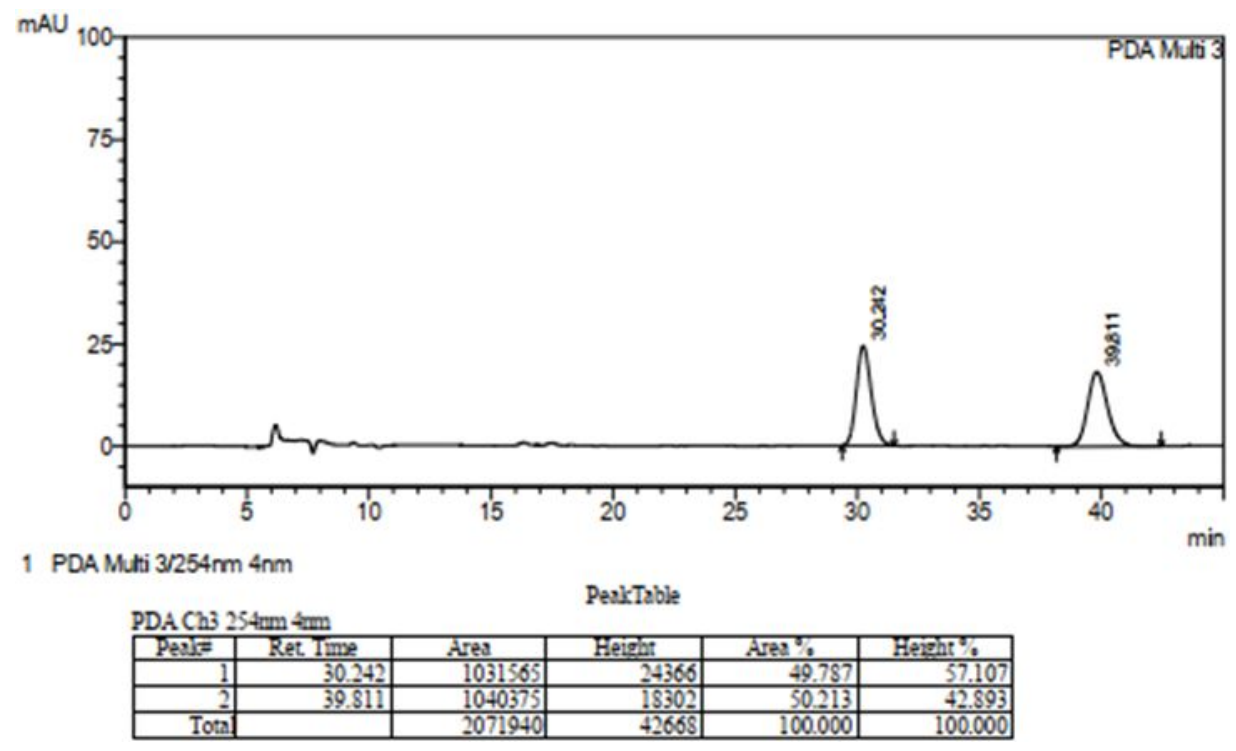




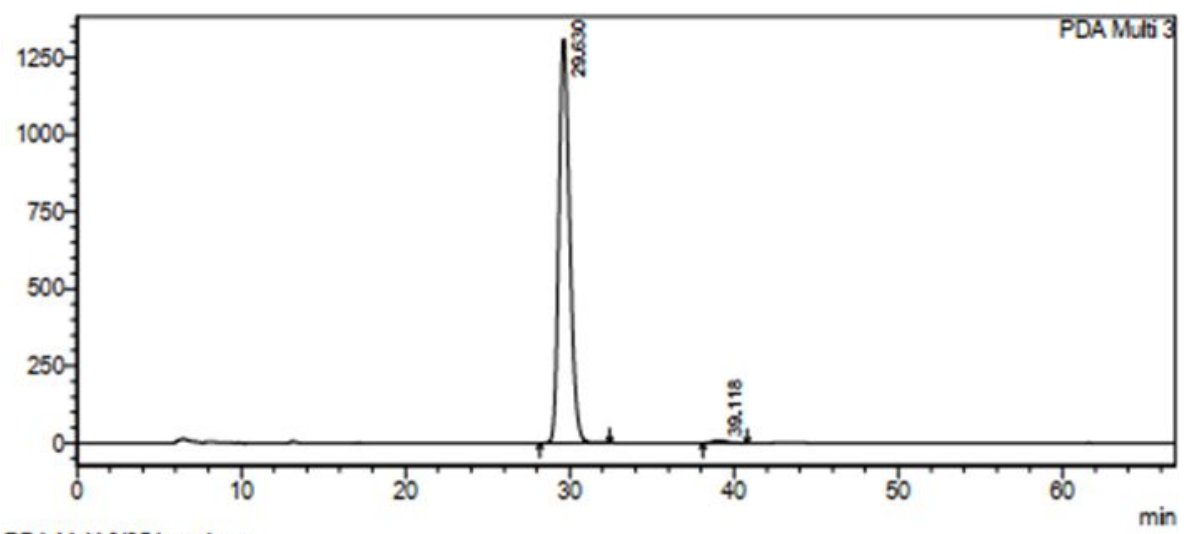

PDA Muti $3 / 254 \mathrm{~mm} 4 \mathrm{~nm}$

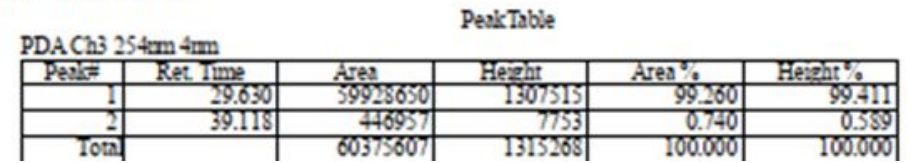

Synthesis of 4-Methoxyphenyl (S)-2-(3-benzamido-1-benzyl-2-oxoindolin-3-yl)ethane-1-sulfonate (8a)<smiles>O=C(N[C@]1(CCS(=O)(=O)F)C(=O)N(Cc2ccccc2)c2ccccc21)c1ccccc1</smiles>

$3 a$

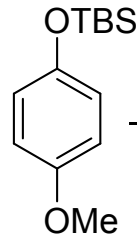

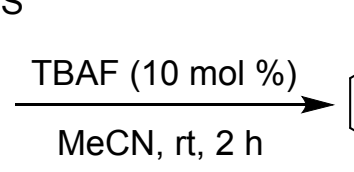<smiles>COc1ccc(OS(=O)(=O)CC[C@H]2C(=O)N(Cc3ccccc3)c3ccccc32)cc1</smiles>

$8 a, 75 \%$ yield, $99 \%$ ee

To a solution of (S)-2-(3-benzamido-1-benzyl-2-oxoindolin-3-yl)ethane-1-sulfonyl fluoride 3a (45.2 mg, $0.1 \mathrm{mmol}), p$-methoxyphenyl TBS ether $(35.7 \mathrm{mg}, 0.15 \mathrm{mmol})$ in $\mathrm{MeCN}(1 \mathrm{~mL})$ was added tetrabutylammonium fluoride (TBAF) $(1.5 \mathrm{mg}, 0.01 \mathrm{mmol})$ at $0{ }^{\circ} \mathrm{C}$. The mixture was stirred at rt until the completion of the reaction $(2 \mathrm{~h})$ as indicated by TLC. After the solvent was evaporated under vacuum, the residue was purified by flash column chromatography over silica gel (Petroleum ether/ethyl acetate $=8: 1)$ to afford the product $8 \mathbf{a}$ as a white solid $(41 \mathrm{mg}, 75 \%)$. m. p. $121.5-123.2{ }^{\circ} \mathrm{C} .{ }^{1} \mathbf{H}$ NMR $(400$ $\left.\mathrm{MHz}, \mathrm{CDCl}_{3}\right) \delta$ 7.86-7.77 (m, 2H), 7.61 (s, 1H), 7.53-7.47 (m, 1H), 7.44-7.37 (m, 4H), 7.36-7.29 (m, $3 \mathrm{H}), 7.30-7.24(\mathrm{~m}, 2 \mathrm{H}), 7.23-7.12(\mathrm{~m}, 3 \mathrm{H}), 7.04(\mathrm{t}, J=7.2 \mathrm{~Hz}, 1 \mathrm{H}), 6.91-6.81(\mathrm{~m}, 2 \mathrm{H}), 6.76(\mathrm{~d}, J=8.0$ $\mathrm{Hz}, 1 \mathrm{H}), 5.04(\mathrm{~d}, J=15.6 \mathrm{~Hz}, 1 \mathrm{H}), 4.96(\mathrm{~d}, J=15.6 \mathrm{~Hz}, 1 \mathrm{H}), 3.83-3.72(\mathrm{~m}, 4 \mathrm{H}), 3.33-3.23(\mathrm{~m}, 1 \mathrm{H})$, 2.87-2.78 (m, 1H), 2.53-2.43 (m, 1H). ${ }^{13} \mathbf{C}$ NMR $\left(101 \mathrm{MHz}, \mathrm{CDCl}_{3}\right) \delta 175.32,166.48,158.62,142.35$, $142.32,135.56,132.51,132.13,129.55,128.93,128.58,127.74,127.45,127.28,123.32,123.06,122.65$, 115.00, 109.81, 59.78, 55.66, 44.37, 43.53, 30.69. HRMS (ESI) $\mathrm{m} / z$ : Calcd. for $\mathrm{C}_{31} \mathrm{H}_{28} \mathrm{~N}_{2} \mathrm{O}_{6} \mathrm{SNa}$, $[\mathrm{M}+\mathrm{Na}]^{+}, 579.1560$; found: 579.1559. $[\alpha]_{\mathrm{D}}{ }^{20}=+24.6\left(\mathrm{c} 1.0, \mathrm{CH}_{2} \mathrm{Cl}_{2}\right)$. Chiral HPLC analysis (Chiral pak AD-H, $i-\operatorname{PrOH} / n$-hexane $=20: 80$, flow rate $=0.5 \mathrm{~mL} / \mathrm{min}$, wave length $=254 \mathrm{~nm}$ ), $\mathrm{t}_{\mathrm{R}}$ (major enantiomer) $=31.981 \mathrm{~min}, \mathrm{t}_{\mathrm{R}}($ minor enantiomer $)=51.900 \mathrm{~min}, 99 \%$ ee. 


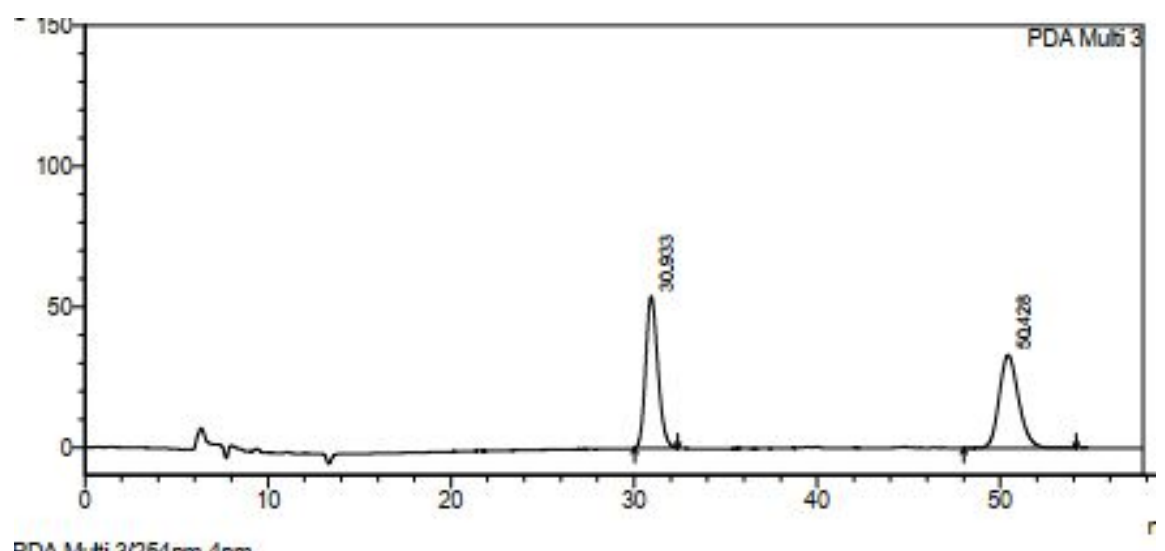

PDA Muti $3 / 254 \mathrm{~mm} 4 \mathrm{~nm}$
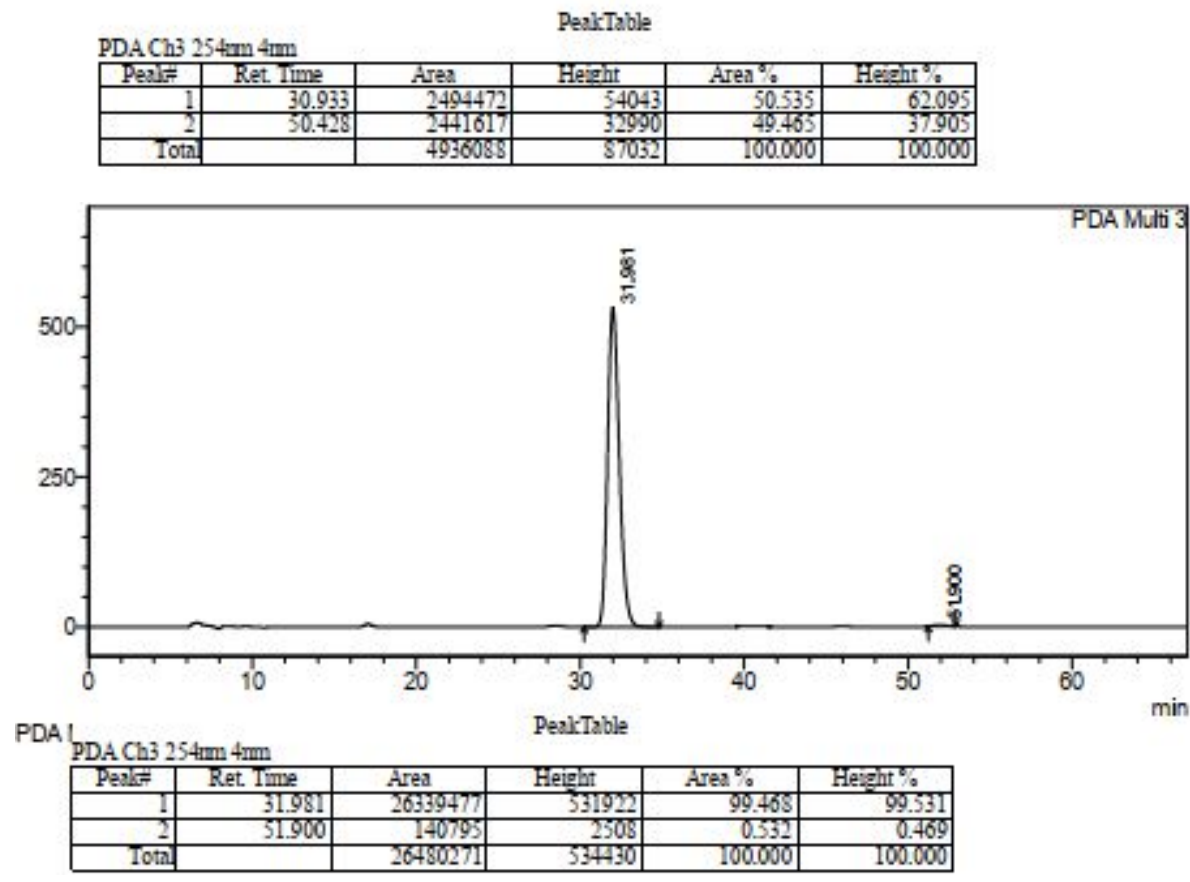

\section{The X-ray crystal data of product $3 v$ and $6 a b$}

CCDC 1952196 (3v) and 1952197 (6ab) contain the supplementary crystallo-graphic data for this paper. These data can be obtained free of charge via www.ccdc.cam.ac.uk/data request/cif<smiles>O=C(NC1(CCS(=O)(=O)F)C(=O)N(Cc2ccccc2)c2ccccc21)C1CC1</smiles>

$3 v$

Identification code

Empirical formula

Formula weight

Temperature/K

Crystal system

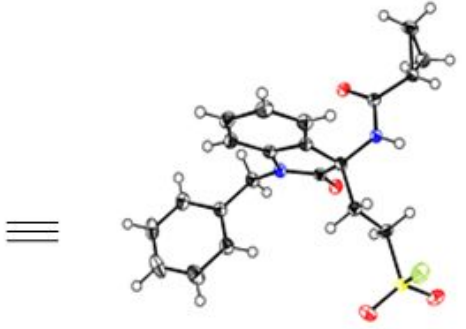

3v CCDC 1952196

$3 \mathrm{v}$

$\mathrm{C}_{21} \mathrm{H}_{21} \mathrm{~N}_{2} \mathrm{O}_{4} \mathrm{SF}$

416.46

100.00(10)

tetragonal 


\begin{tabular}{|c|c|}
\hline Space group & $\mathrm{P}_{3}$ \\
\hline $\mathrm{a} / \AA$ & $11.15080(10)$ \\
\hline $\mathrm{b} / \AA$ & $11.15080(10)$ \\
\hline $\mathrm{c} / \AA ̊$ & $16.05760(10)$ \\
\hline$\alpha /^{\circ}$ & 90 \\
\hline$\beta /{ }^{\circ}$ & 90 \\
\hline$\gamma /{ }^{\circ}$ & 90 \\
\hline Volume $/ \AA^{3}$ & $1996.61(4)$ \\
\hline $\mathrm{Z}$ & 4 \\
\hline$\rho_{\text {calc }} \mathrm{g} / \mathrm{cm}^{3}$ & 1.385 \\
\hline$\mu / \mathrm{mm}^{-1}$ & 1.789 \\
\hline $\mathrm{F}(000)$ & 872.0 \\
\hline Crystal size $/ \mathrm{mm}^{3}$ & $0.3 \times 0.2 \times 0.1$ \\
\hline Radiation & $\mathrm{CuK} \alpha(\lambda=1.54184)$ \\
\hline $2 \Theta$ range for data collection ${ }^{\circ}$ & 7.928 to 153.86 \\
\hline Index ranges & $-14 \leq \mathrm{h} \leq 14,-12 \leq \mathrm{k} \leq 13,-19 \leq 1 \leq 19$ \\
\hline Reflections collected & 20194 \\
\hline Independent reflections & $4121\left[R_{\text {int }}=0.0251, R_{\text {sigma }}=0.0156\right]$ \\
\hline Data/restraints/parameters & $4121 / 1 / 262$ \\
\hline Goodness-of-fit on $\mathrm{F}^{2}$ & 1.066 \\
\hline Final $\mathrm{R}$ indexes $[\mathrm{I}>=2 \sigma(\mathrm{I})]$ & $\mathrm{R}_{1}=0.0256, \mathrm{wR}_{2}=0.0667$ \\
\hline Final $\mathrm{R}$ indexes [all data] & $\mathrm{R}_{1}=0.0260, \mathrm{wR}_{2}=0.0680$ \\
\hline Largest diff. peak/hole / e $\AA^{-3}$ & $0.18 /-0.29$ \\
\hline Flack parameter & $0.000(5)$ \\
\hline $6 a b$ & CCDC 1952197 \\
\hline Identification code & $6 a b$ \\
\hline Empirical formula & $\mathrm{C}_{30} \mathrm{H}_{24} \mathrm{~N}_{2} \mathrm{O}_{4} \mathrm{~S}$ \\
\hline Formula weight & 508.57 \\
\hline Temperature/K & $100.00(10)$ \\
\hline Crystal system & orthorhombic \\
\hline Space group & $\mathrm{P} 2{ }_{1} 2_{1} 2_{1}$ \\
\hline $\mathrm{a} / \AA \AA$ & $7.92191(4)$ \\
\hline $\mathrm{b} / \AA \AA$ & $12.56234(7)$ \\
\hline $\mathrm{c} / \AA ̊$ & $25.20069(13)$ \\
\hline & S63 /S143 \\
\hline
\end{tabular}




\begin{tabular}{|c|c|}
\hline$\alpha /^{\circ}$ & 90 \\
\hline$\beta /{ }^{\circ}$ & 90 \\
\hline$\gamma /{ }^{\circ}$ & 90 \\
\hline Volume $/ \AA^{3}$ & $2507.92(2)$ \\
\hline $\mathrm{Z}$ & 4 \\
\hline$\rho_{\text {calc }} \mathrm{g} / \mathrm{cm}^{3}$ & 1.347 \\
\hline$\mu / \mathrm{mm}^{-1}$ & 1.474 \\
\hline $\mathrm{F}(000)$ & 1064.0 \\
\hline Crystal size $/ \mathrm{mm}^{3}$ & $0.2 \times 0.1 \times 0.1$ \\
\hline Radiation & $\operatorname{CuK} \alpha(\lambda=1.54184)$ \\
\hline $2 \Theta$ range for data collection ${ }^{\circ}$ & 7.016 to 154.038 \\
\hline Index ranges & $-7 \leq \mathrm{h} \leq 9,-15 \leq \mathrm{k} \leq 15,-30 \leq 1 \leq 31$ \\
\hline Reflections collected & 25706 \\
\hline Independent reflections & $5122\left[\mathrm{R}_{\mathrm{int}}=0.0306, \mathrm{R}_{\text {sigma }}=0.0197\right]$ \\
\hline Data/restraints/parameters & $5122 / 0 / 335$ \\
\hline Goodness-of-fit on $\mathrm{F}^{2}$ & 1.047 \\
\hline Final $R$ indexes $[\mathrm{I}>=2 \sigma(\mathrm{I})]$ & $\mathrm{R}_{1}=0.0246, \mathrm{wR}_{2}=0.0625$ \\
\hline Final $\mathrm{R}$ indexes [all data] & $\mathrm{R}_{1}=0.0259, \mathrm{wR}_{2}=0.0633$ \\
\hline Largest diff. peak/hole / e $\AA^{-3}$ & $0.20 /-0.26$ \\
\hline Flack parameter & $0.011(4)$ \\
\hline
\end{tabular}

\section{References}

1) Pei, C. K.; Jiang, Y.; Shi, M. Eur. J. Org. Chem. 2012, 2012, 4206-4216.

2) Zheng, Q.; Dong, J.; Sharpless, K. B. J. Org. Chem. 2016, 81, 11360-11362.

3) Zha, G. F.; Zheng, Q.; Leng, J.; Wu, P.; Qin, H. L.; Sharpless, K. B. Angew. Chem., Int. Ed. 2017, $56,4849-4852$.

4) Zha, G. F.; Bare, G. A. L.; Leng, J.; Shang, Z. P.; Luo, Z.; Qin, H. L. Adv. Synth. Catal. 2017, 359, 3237-3242.

5) Wang, J.; Li, H.; Zu, L.; Jiang, W.; Xie, H.; Duan, W.; Wang, W. J. Am. Chem. Soc. 2006, 128, 12652-12653.

6) Wang, Y. Q.; Song, J.; Hong, R.; Li, H.; Deng, L. J. Am. Chem. Soc. 2006, 128, 8156-8157.

7) Badiola, E.; Fiser, B.; Gomez Bengoa, E.; Mielgo, A.; Olaizola, I.; Urruzuno, I.; Garcia, J. M.; Odriozola, J. M.; Razkin, J.; Oiarbide, M.; Palomo, C. J. Am. Chem. Soc. 2014, 136, 17869-17881.

8) Porta, R.; Benaglia, M.; Coccia, F.; Cozzi, F.; Puglisi, A. Adv. Synth. Catal. 2015, 357, 377-383.

9) Rout, S.; Joshi, H.; Singh, V. K. Org. Lett. 2018, 20, 2199-2203. 


\section{NMR spectra}

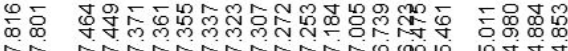

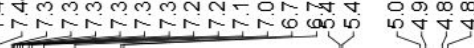

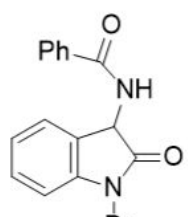

$1 \mathrm{a}$

$\mathrm{Bn}$
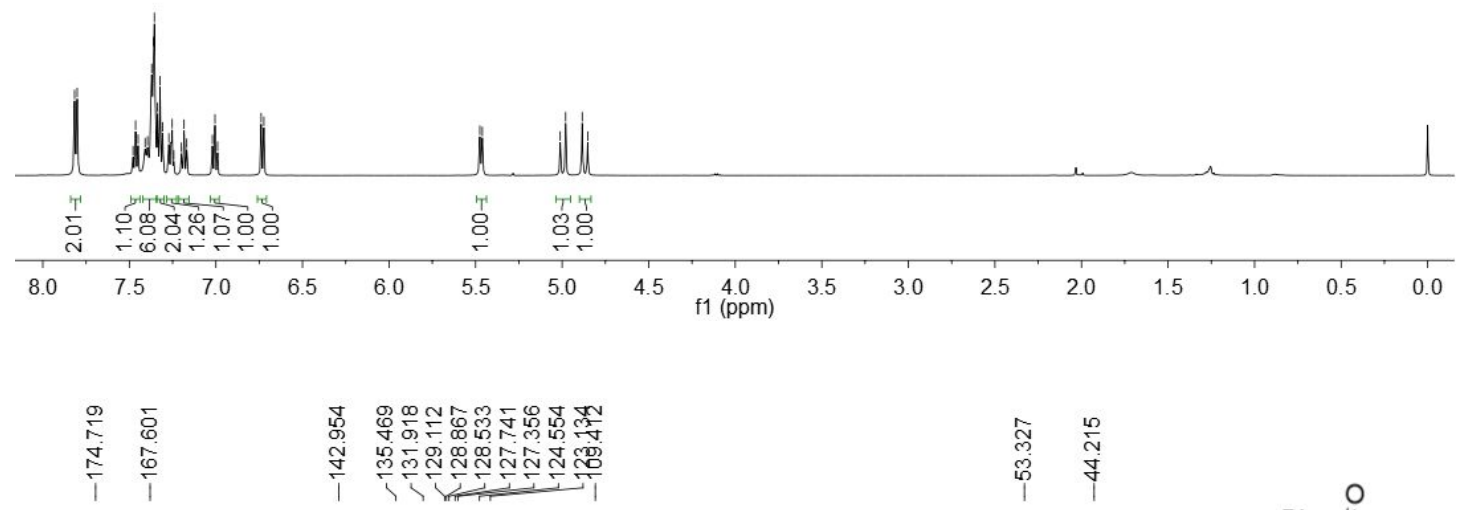

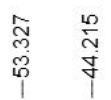

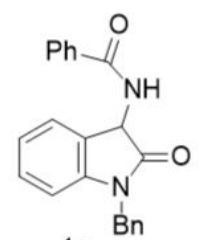

1a

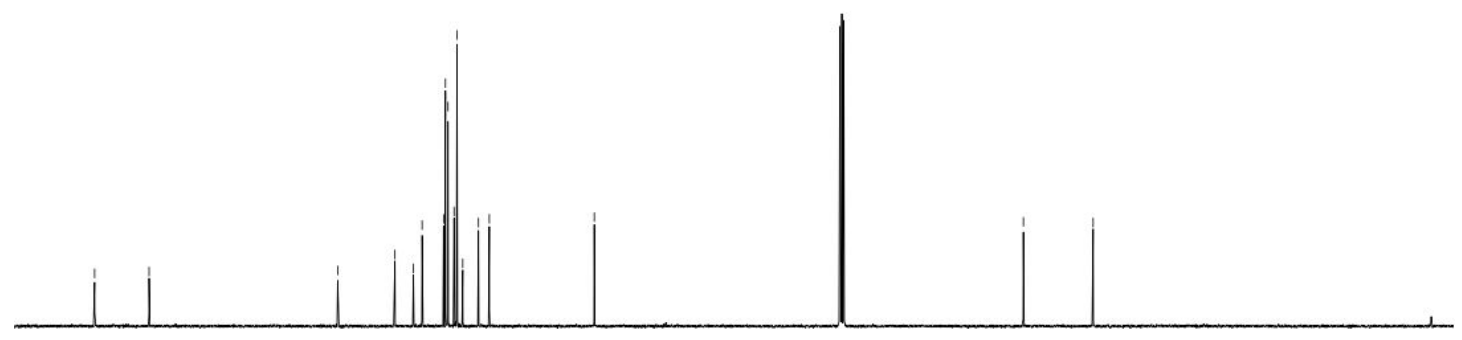

$\begin{array}{lllllllllllllllllll}180 & 170 & 160 & 150 & 140 & 130 & 120 & 110 & 100 & 90 & 80 & 70 & 60 & 50 & 40 & 30 & 20 & 10 & 0\end{array}$

${ }^{1} \mathrm{H}$ and ${ }^{13} \mathrm{C}$ NMR spectra of $\mathbf{1 a}$ 


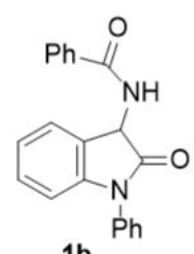

1b
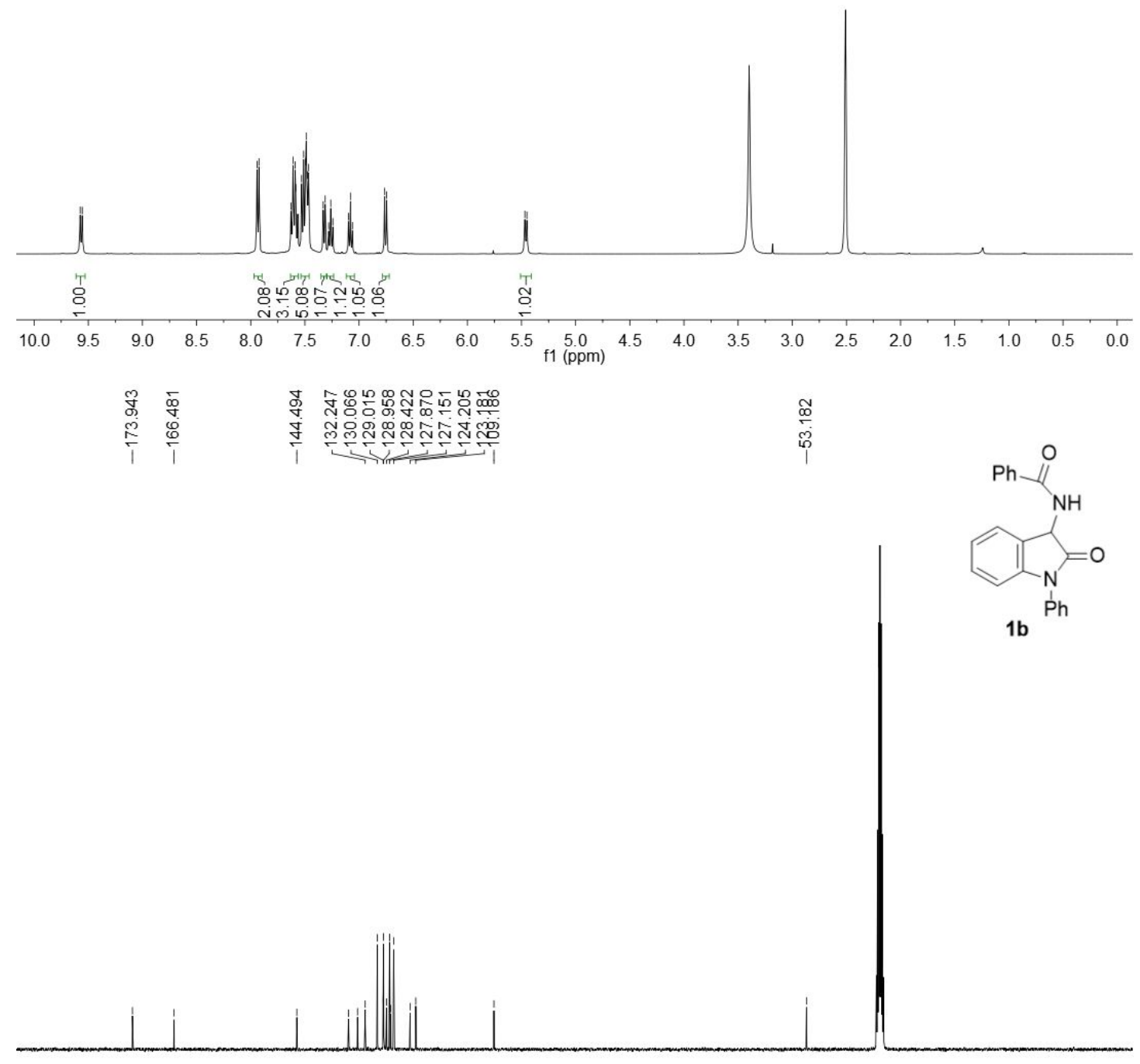

$\begin{array}{llllllllllllllllllll}190 & 180 & 170 & 160 & 150 & 140 & 130 & 120 & 110 & \begin{array}{c}100 \\ \mathrm{f} 1(\mathrm{ppm})\end{array} & 80 & 70 & 60 & 50 & 40 & 30 & 20 & 10 & 0\end{array}$

${ }^{1} \mathrm{H}$ and ${ }^{13} \mathrm{C}$ NMR spectra of $\mathbf{1 b}$ 

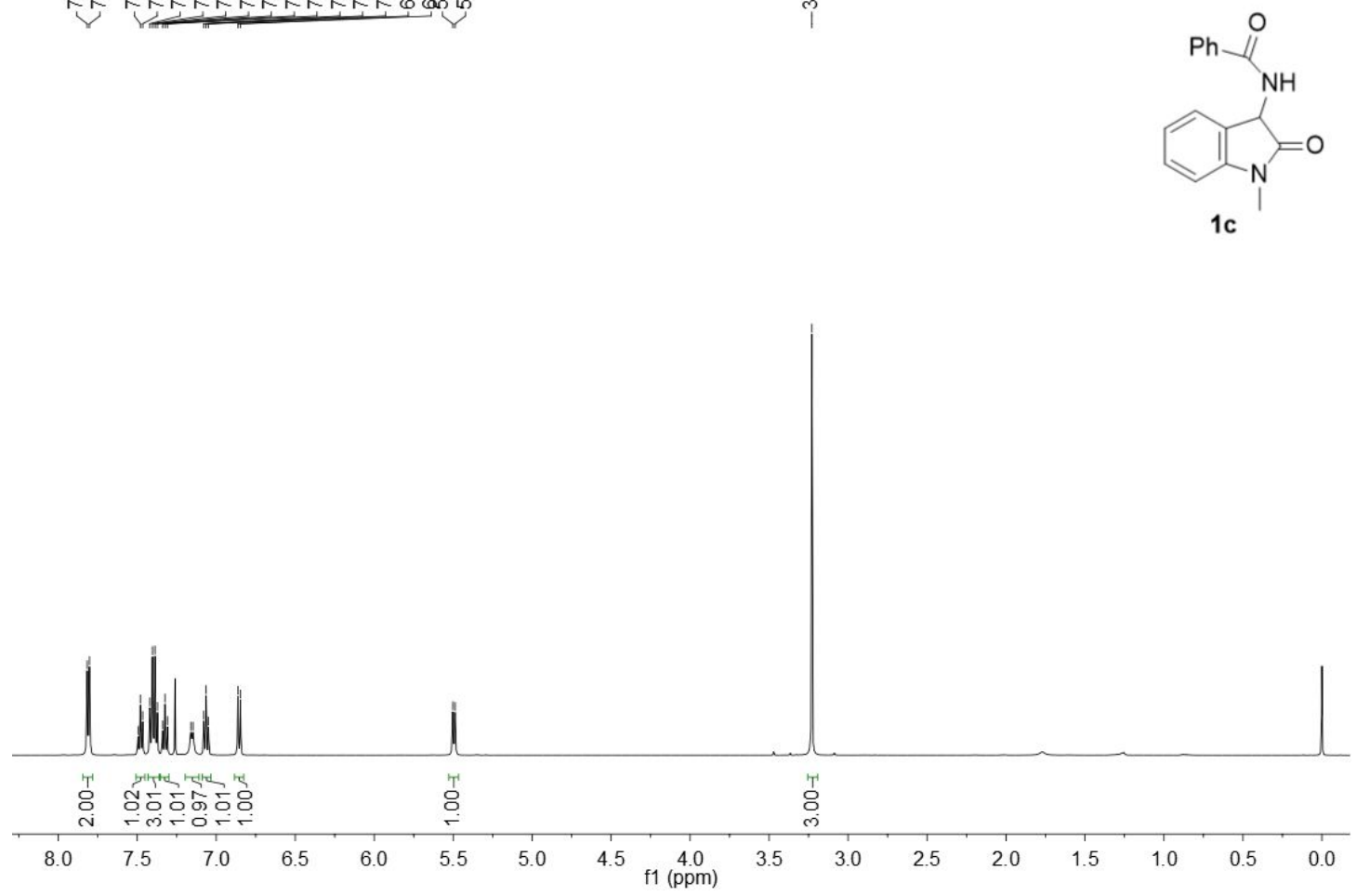

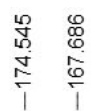

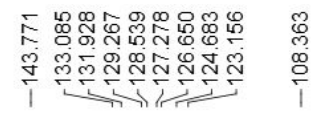

$\underset{\substack{i \\ \frac{0}{7}}}{7}$

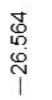
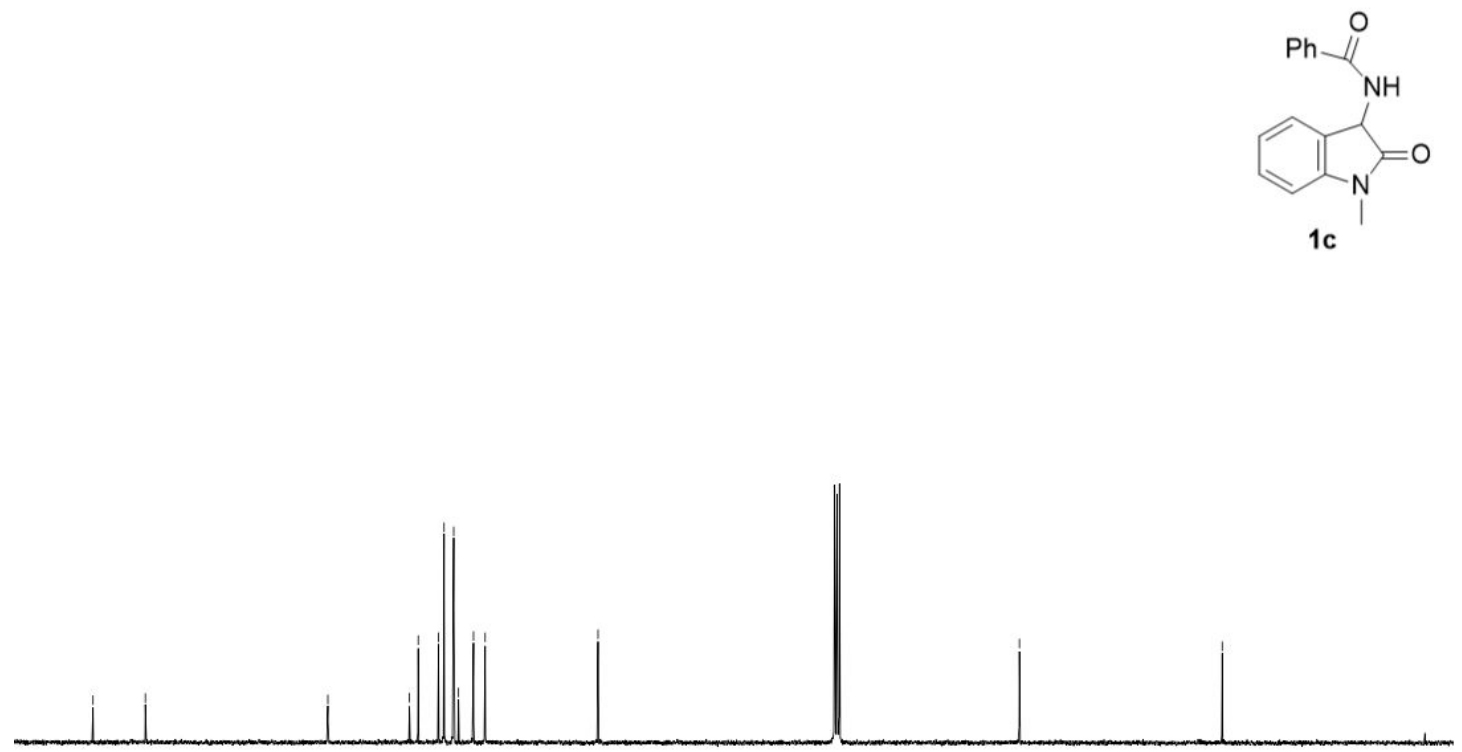

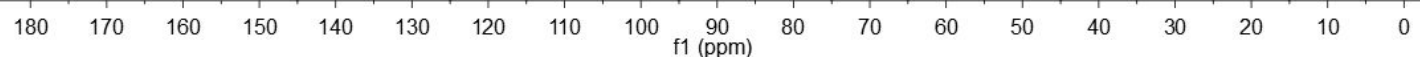

${ }^{1} \mathrm{H}$ and ${ }^{13} \mathrm{C}$ NMR spectra of $\mathbf{1 c}$ 


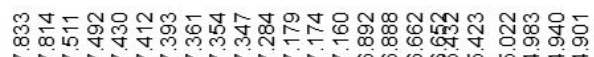

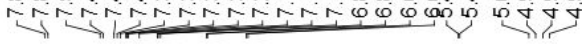

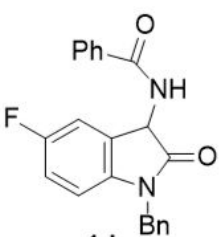

1d ${ }^{\mathrm{Bn}}$
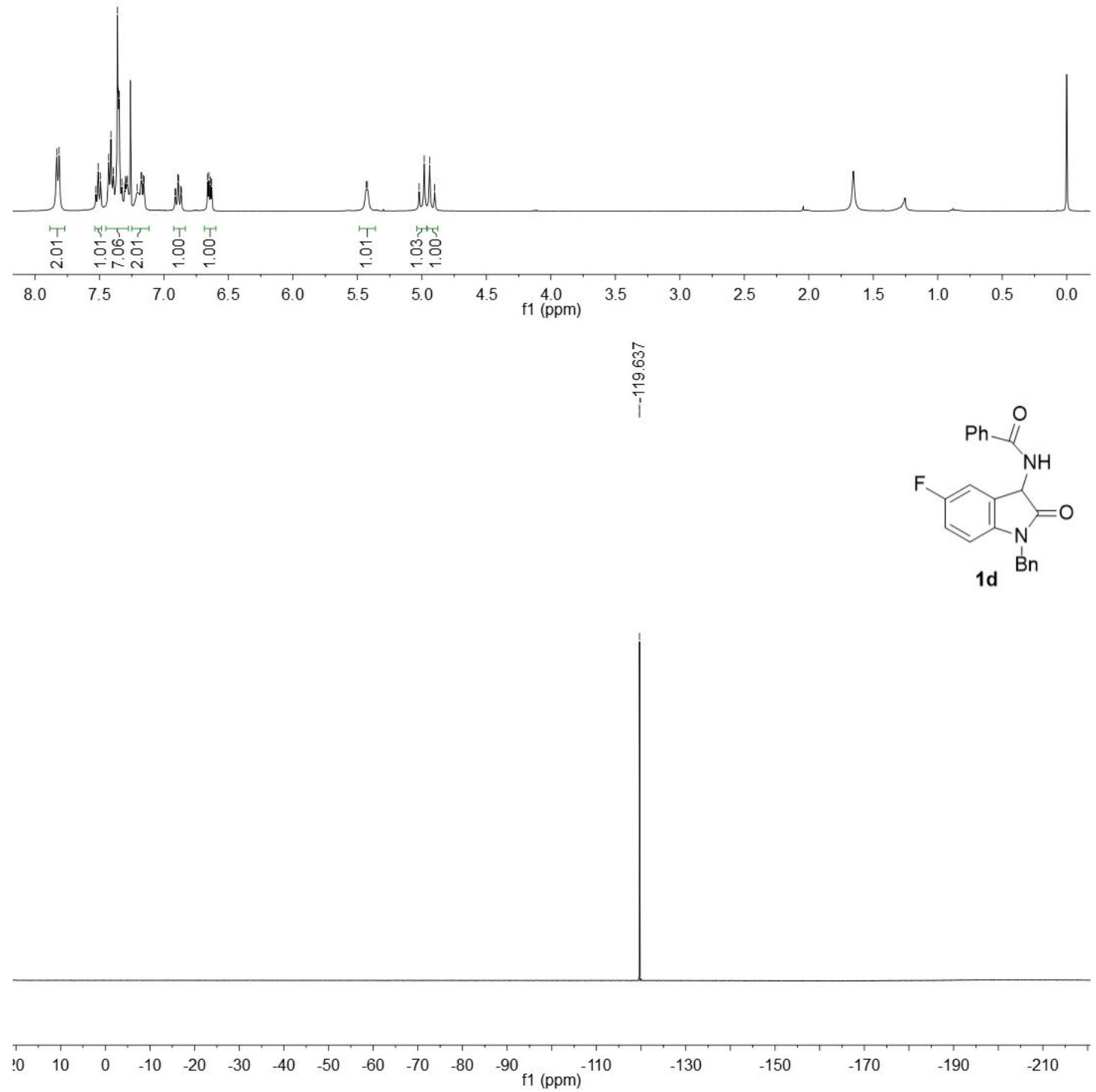


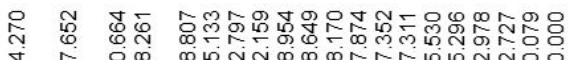

i

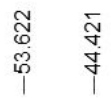
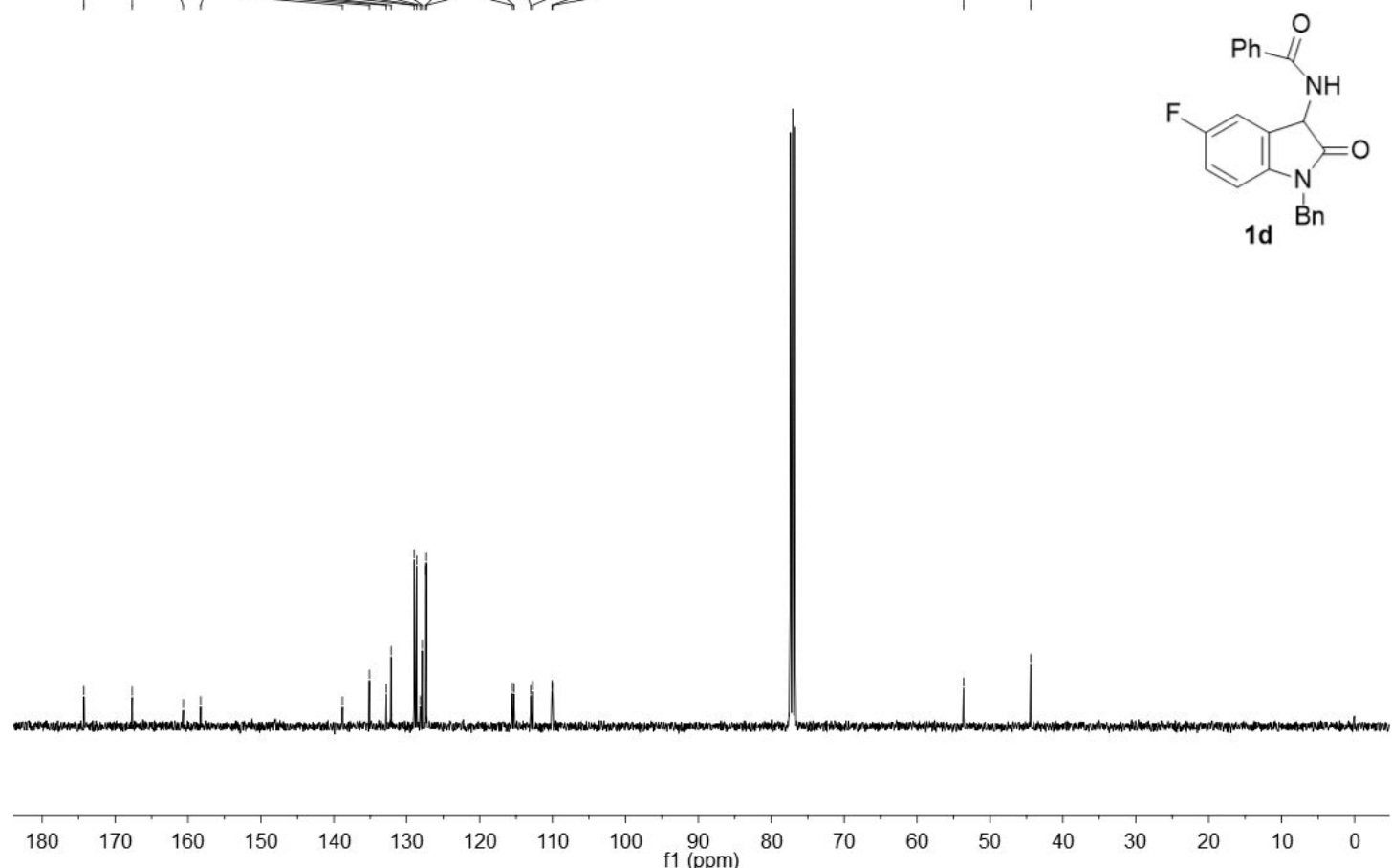

${ }^{1} \mathrm{H},{ }^{19} \mathrm{~F}$ and ${ }^{13} \mathrm{C}$ NMR spectra of $\mathbf{1 d}$

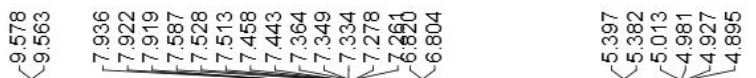
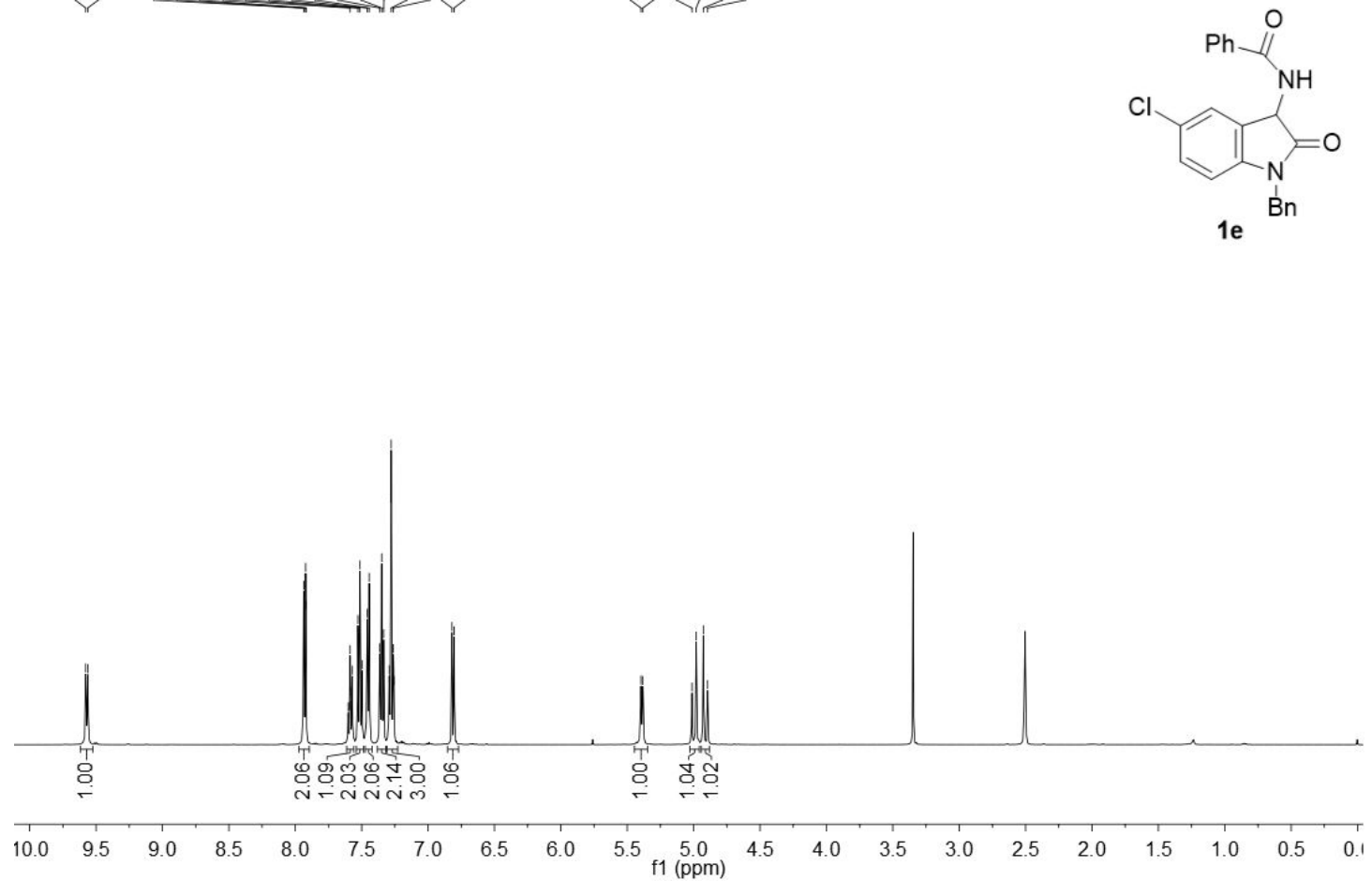


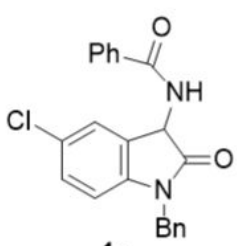

$1 e$

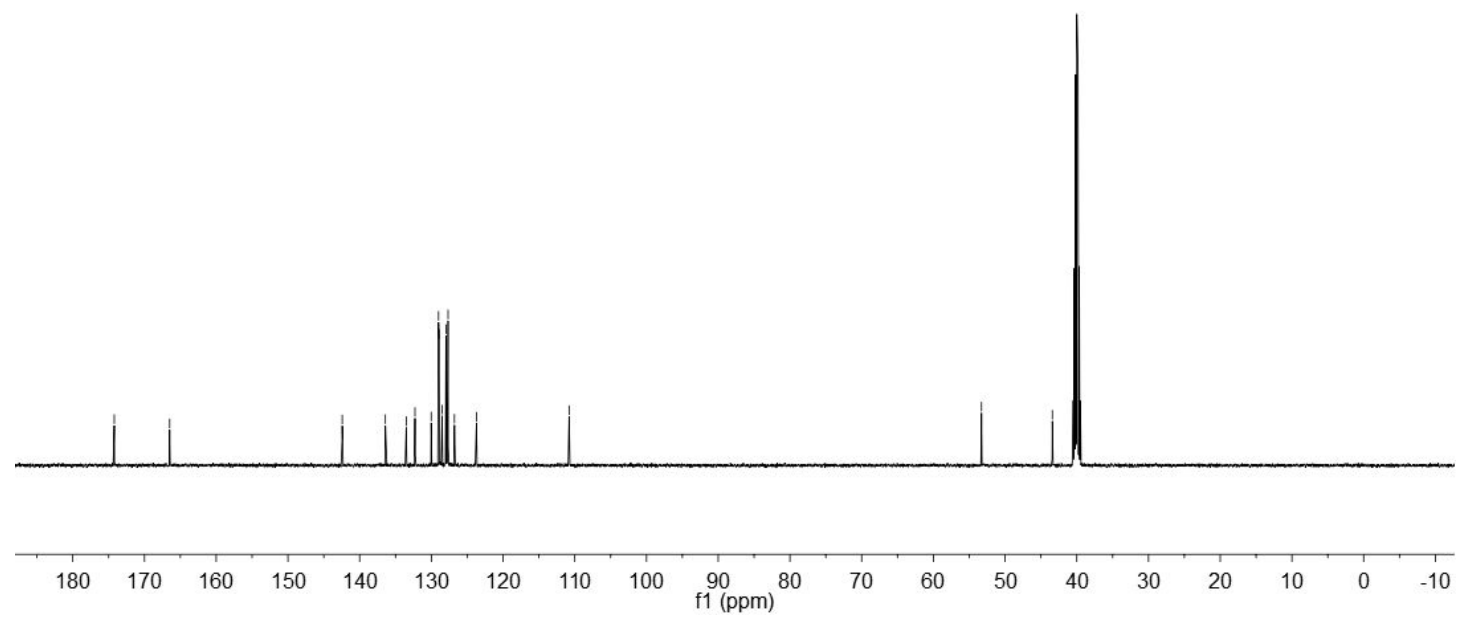

${ }^{1} \mathrm{H}$ and ${ }^{13} \mathrm{C}$ NMR spectra of $\mathbf{1 e}$

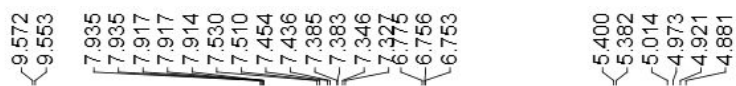
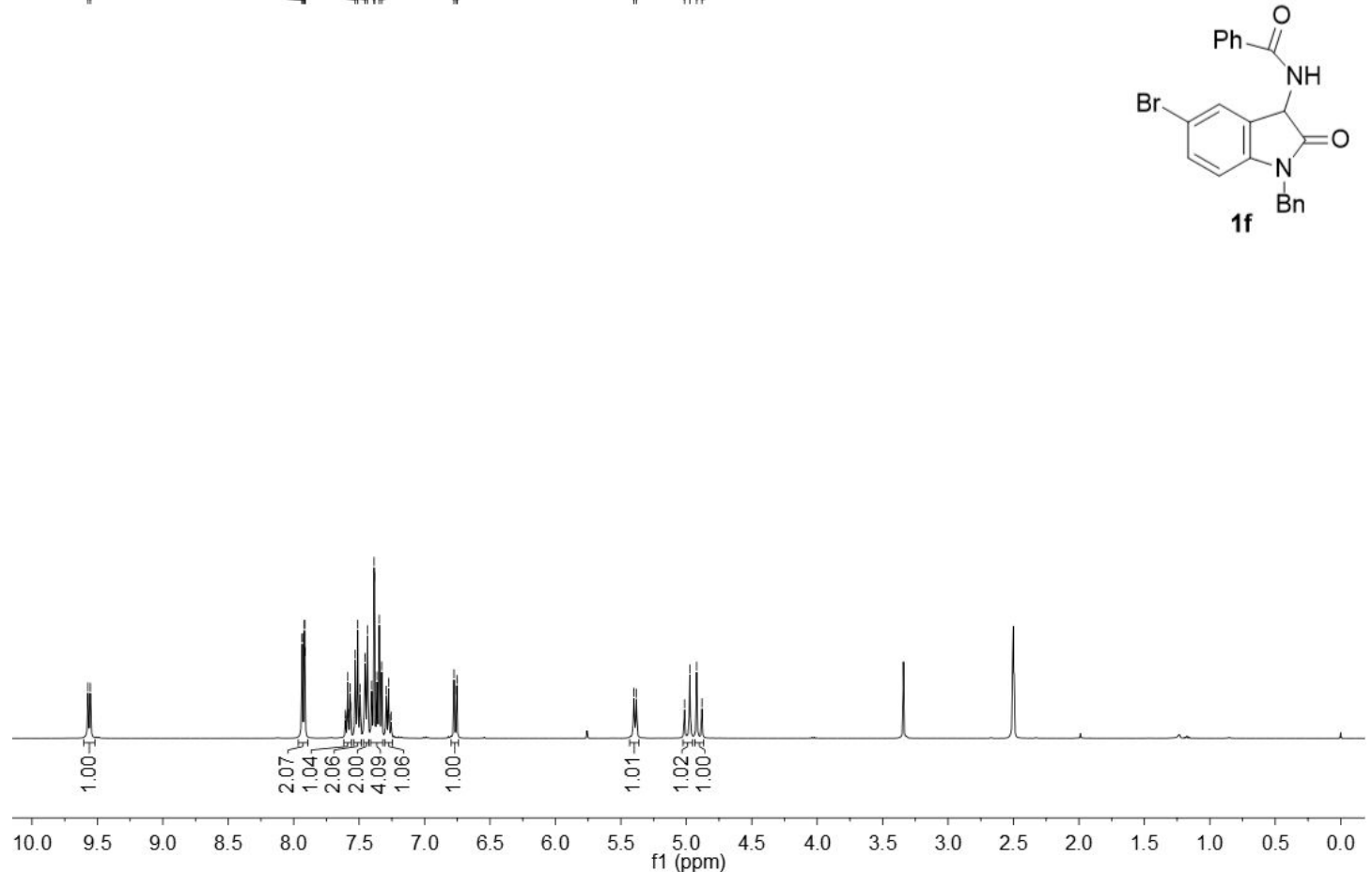


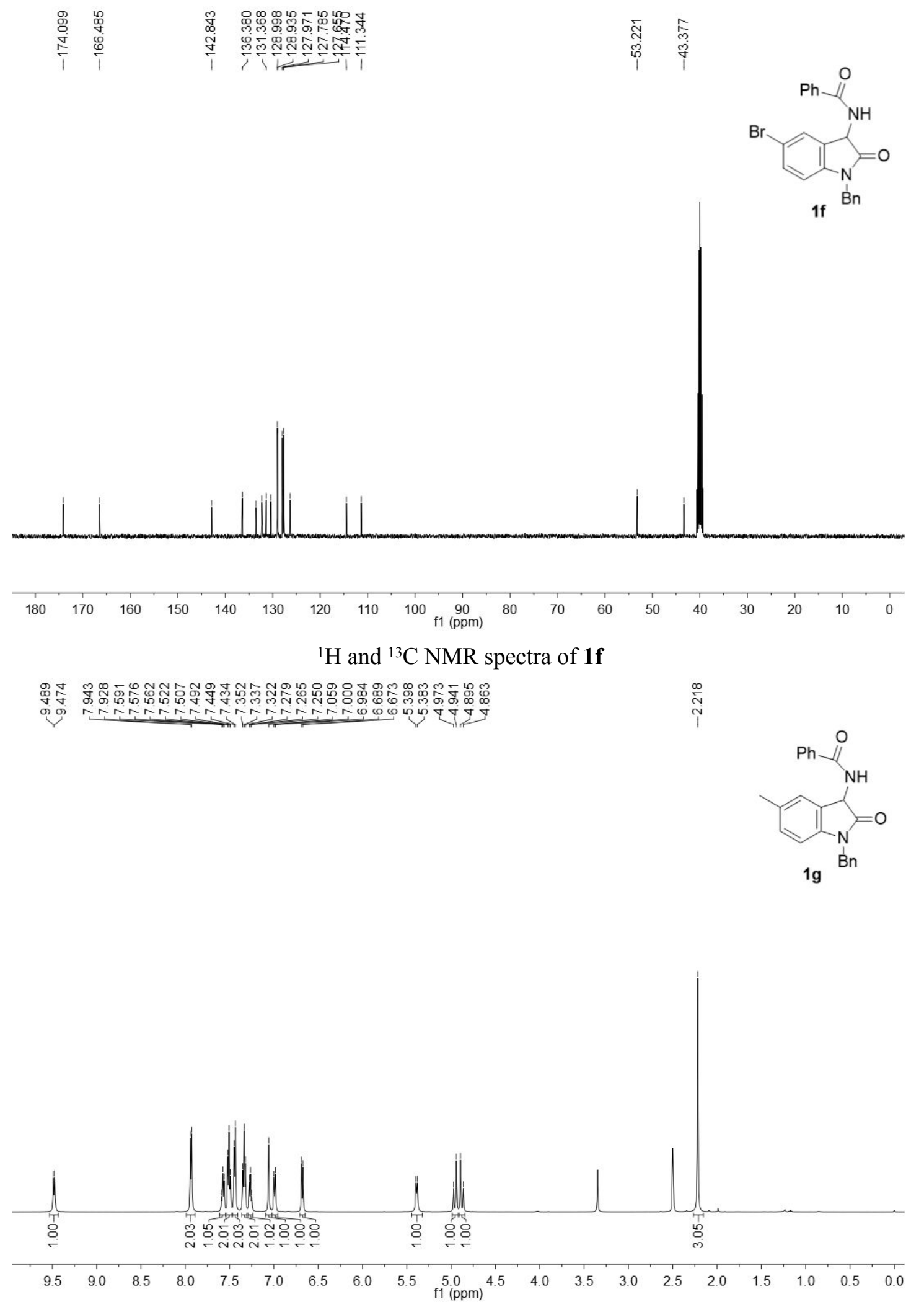




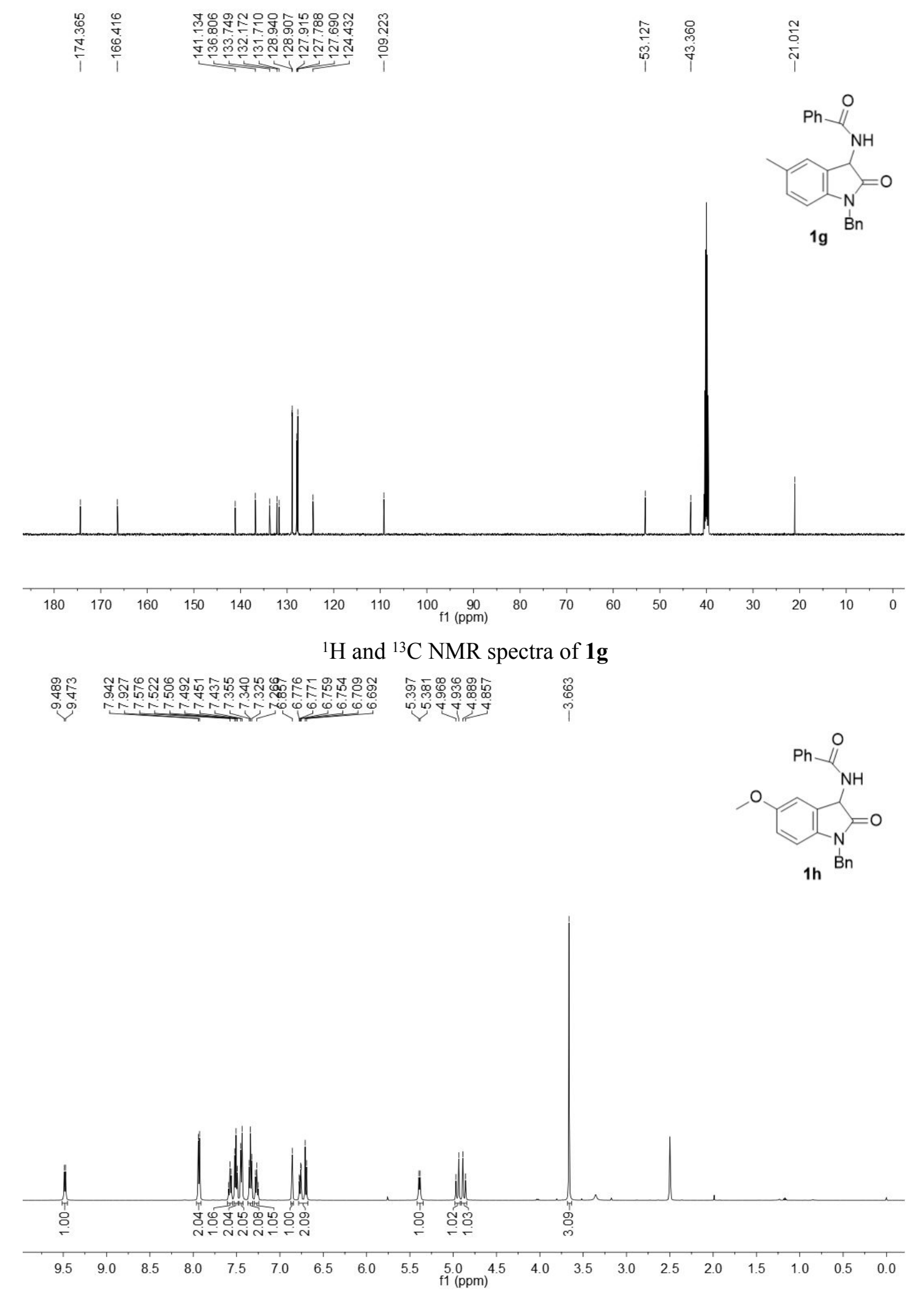




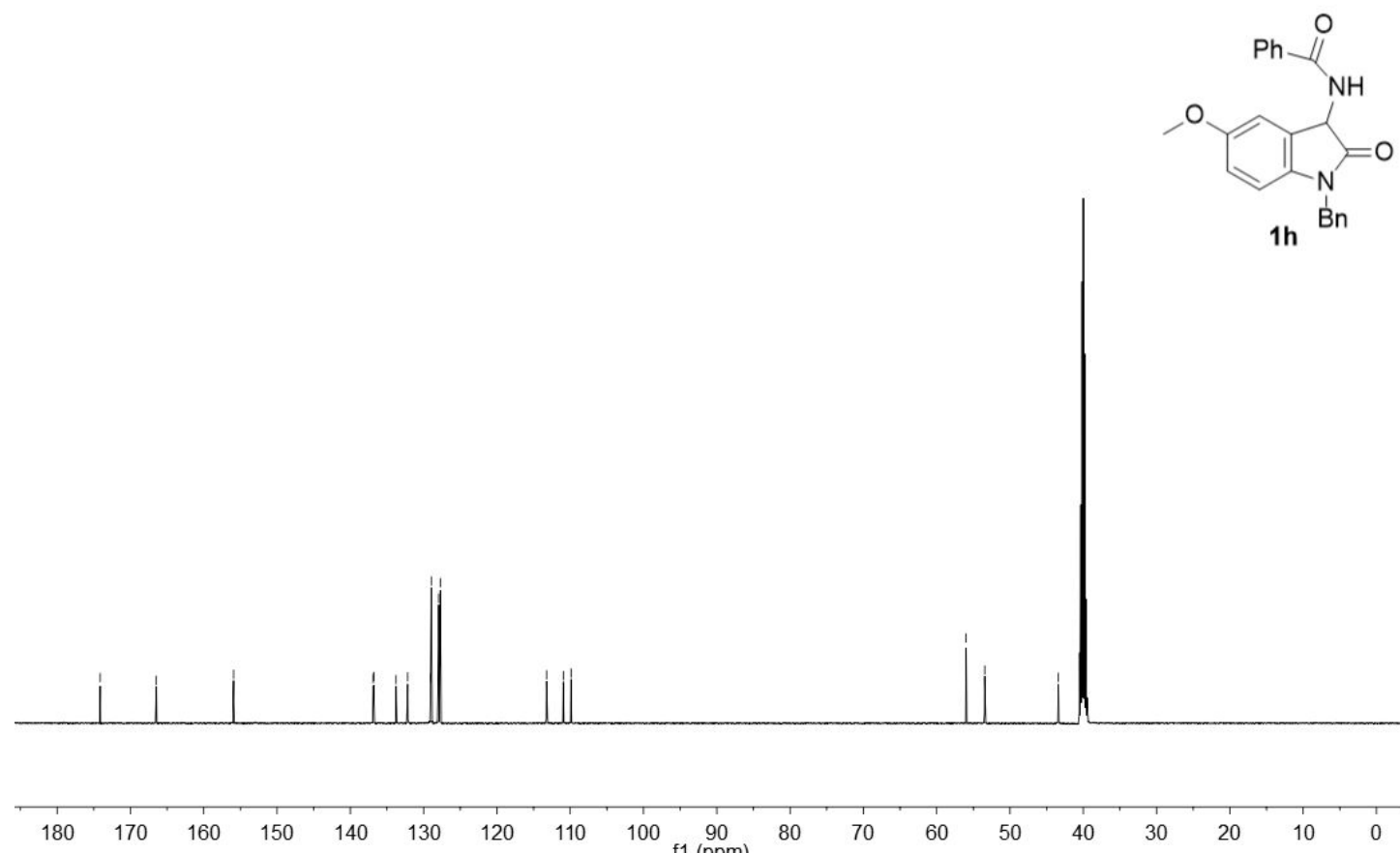

${ }^{1} \mathrm{H}$ and ${ }^{13} \mathrm{C}$ NMR spectra of $\mathbf{1 h}$

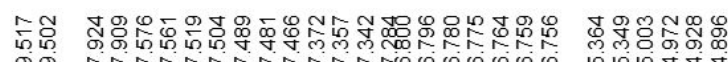

oj
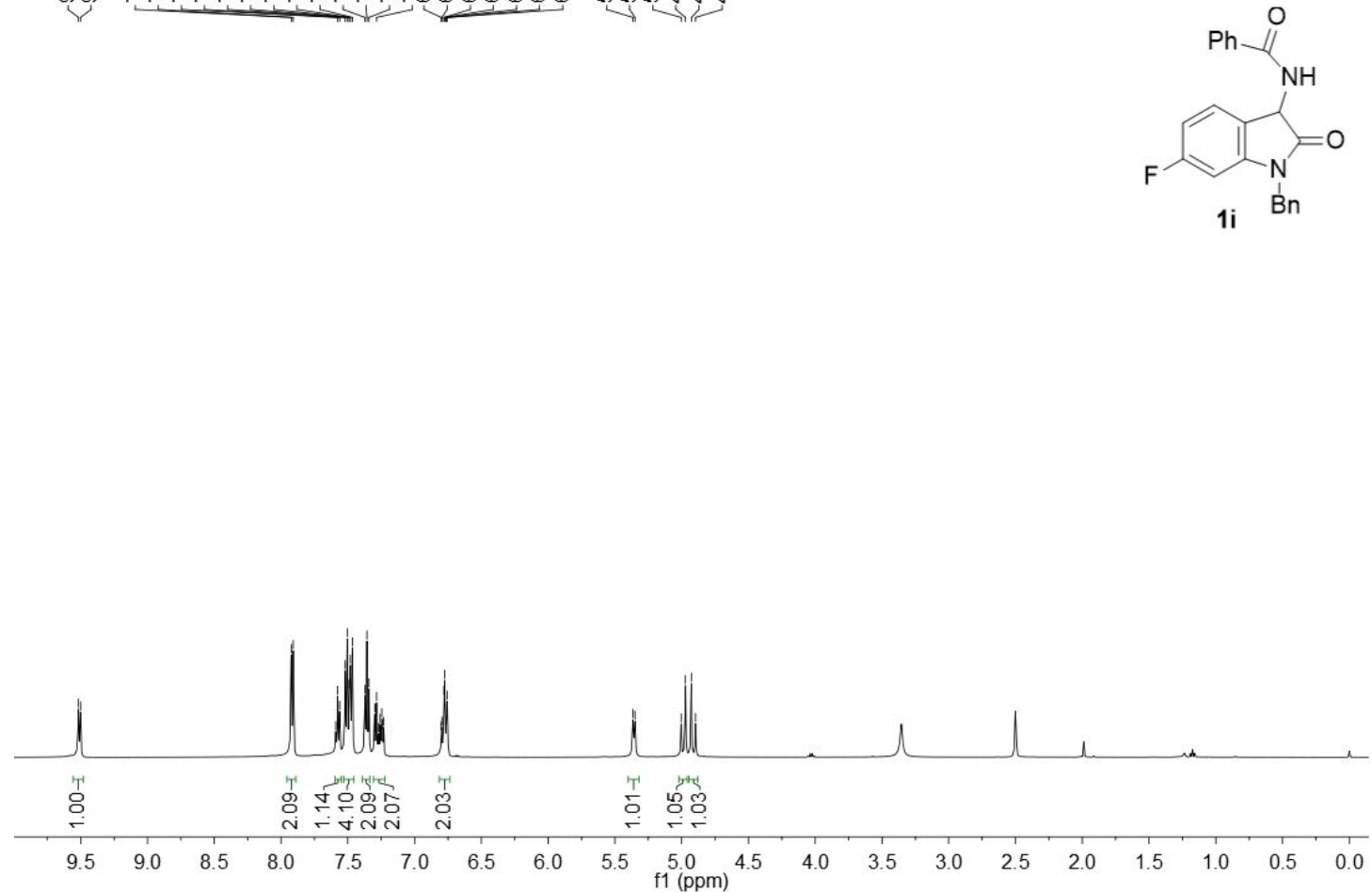


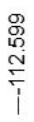
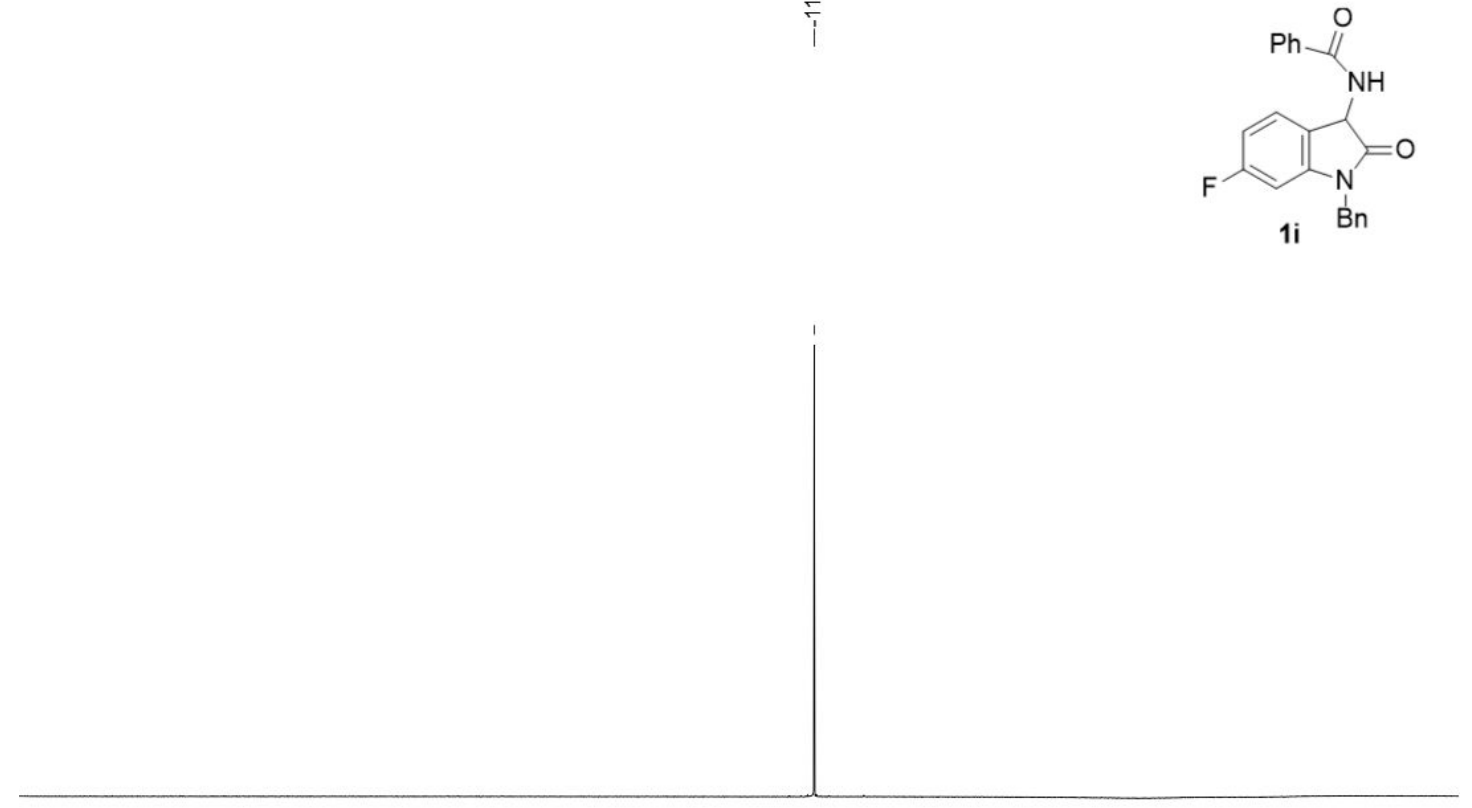

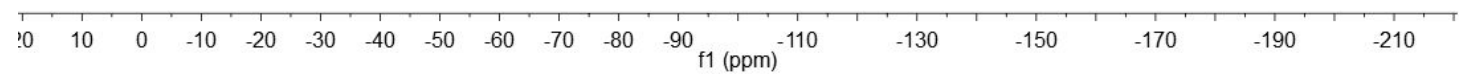

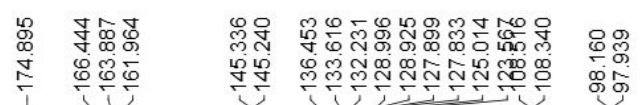

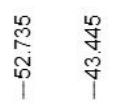
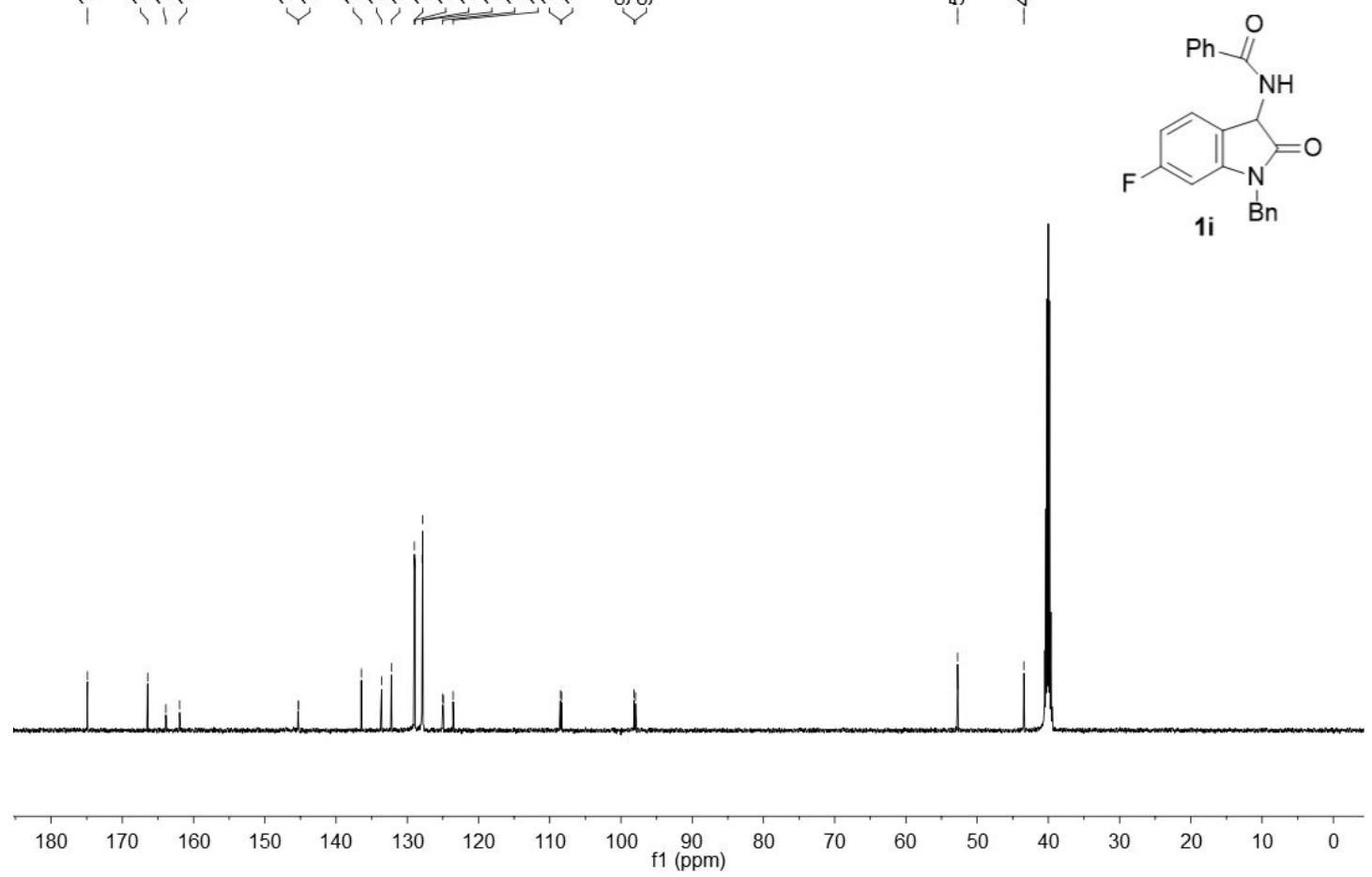

${ }^{1} \mathrm{H},{ }^{19} \mathrm{~F}$ and ${ }^{13} \mathrm{C}$ NMR spectra of $\mathbf{1 i}$ 


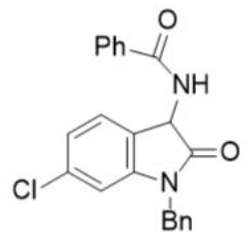

1j

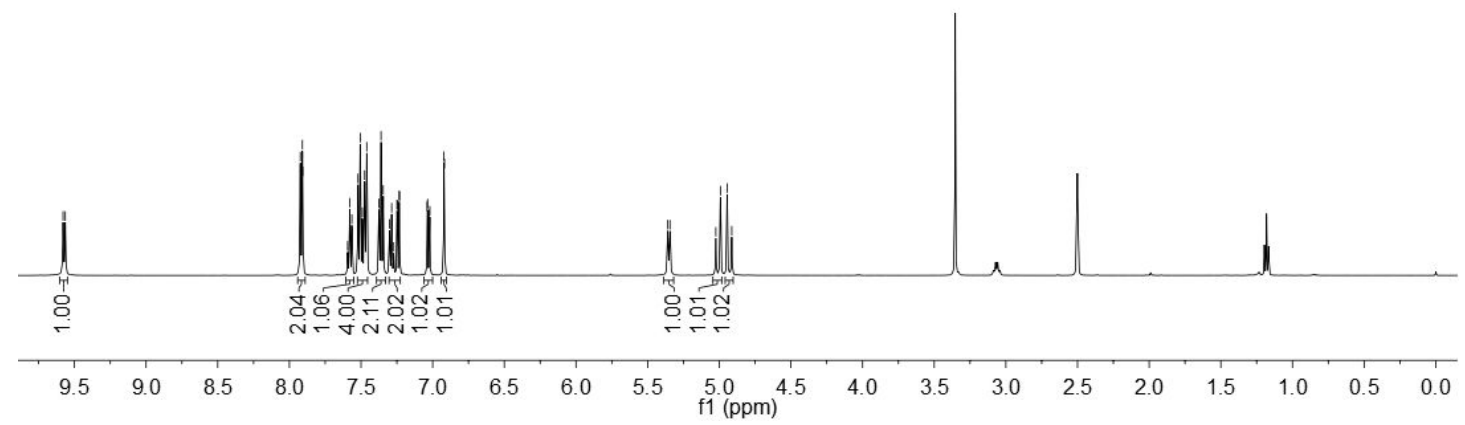

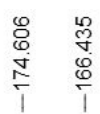

ำ

药

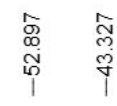

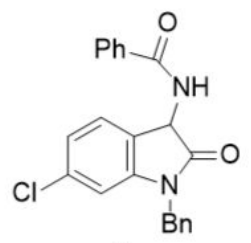

1j

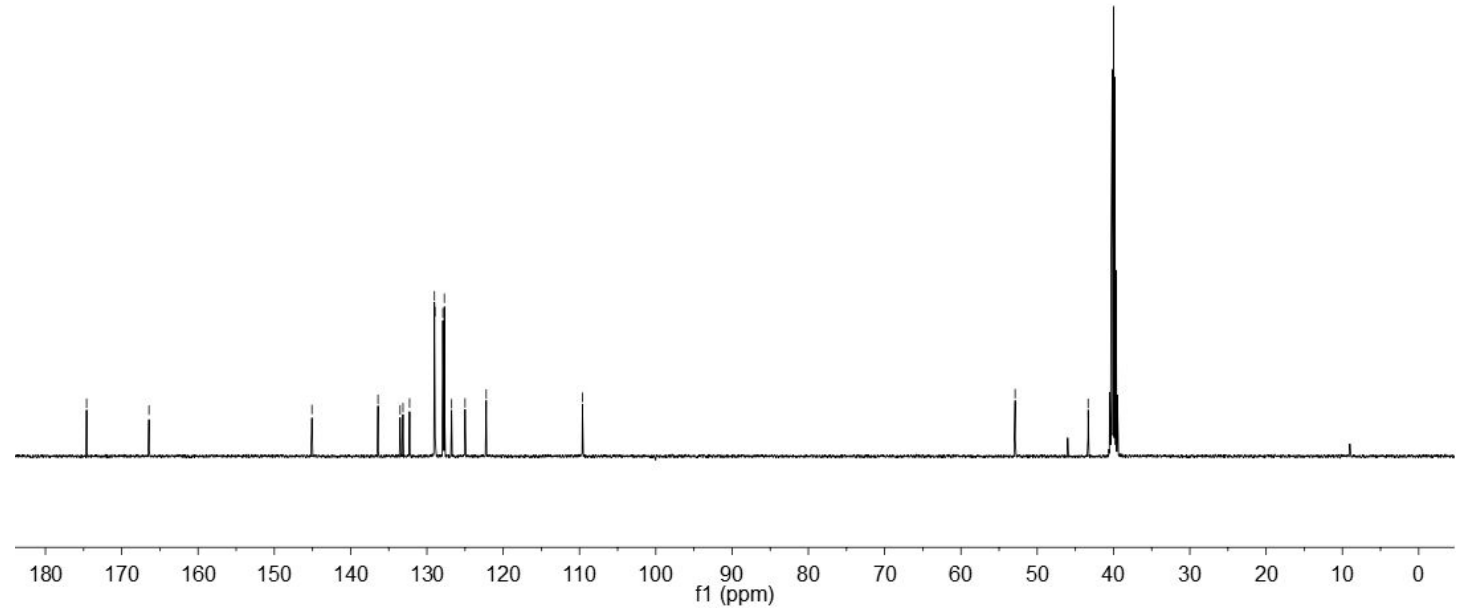

${ }^{1} \mathrm{H}$ and ${ }^{13} \mathrm{C}$ NMR spectra of $\mathbf{1} \mathbf{j}$ 

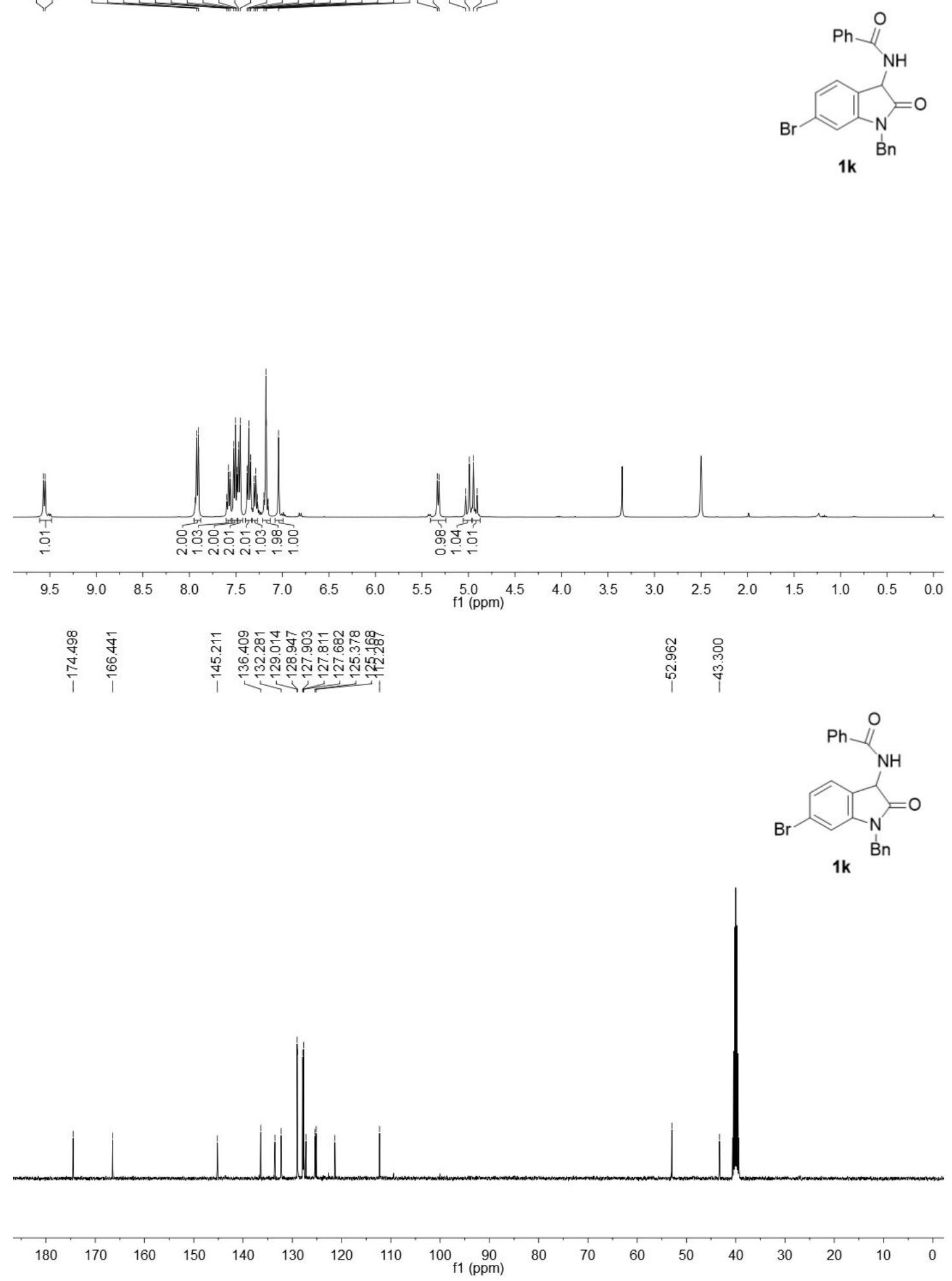

${ }^{1} \mathrm{H}$ and ${ }^{13} \mathrm{C}$ NMR spectra of $\mathbf{1 k}$ 


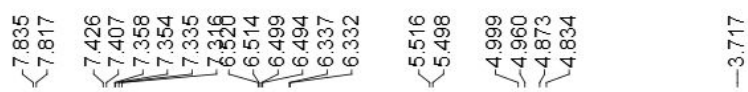
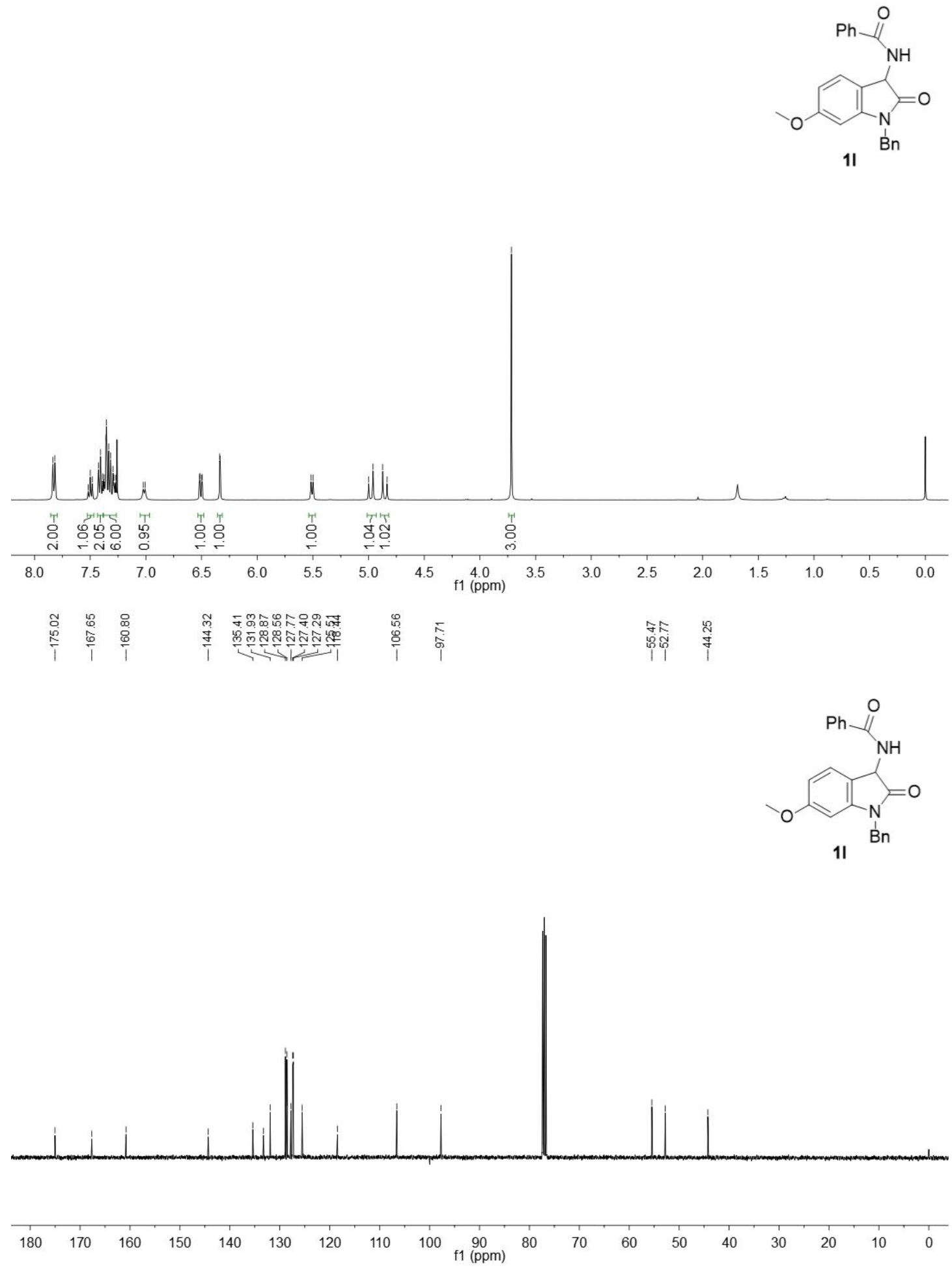

${ }^{1} \mathrm{H}$ and ${ }^{13} \mathrm{C}$ NMR spectra of $\mathbf{1 l}$ 


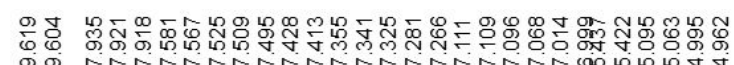

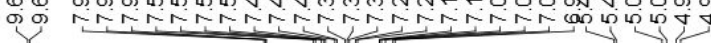

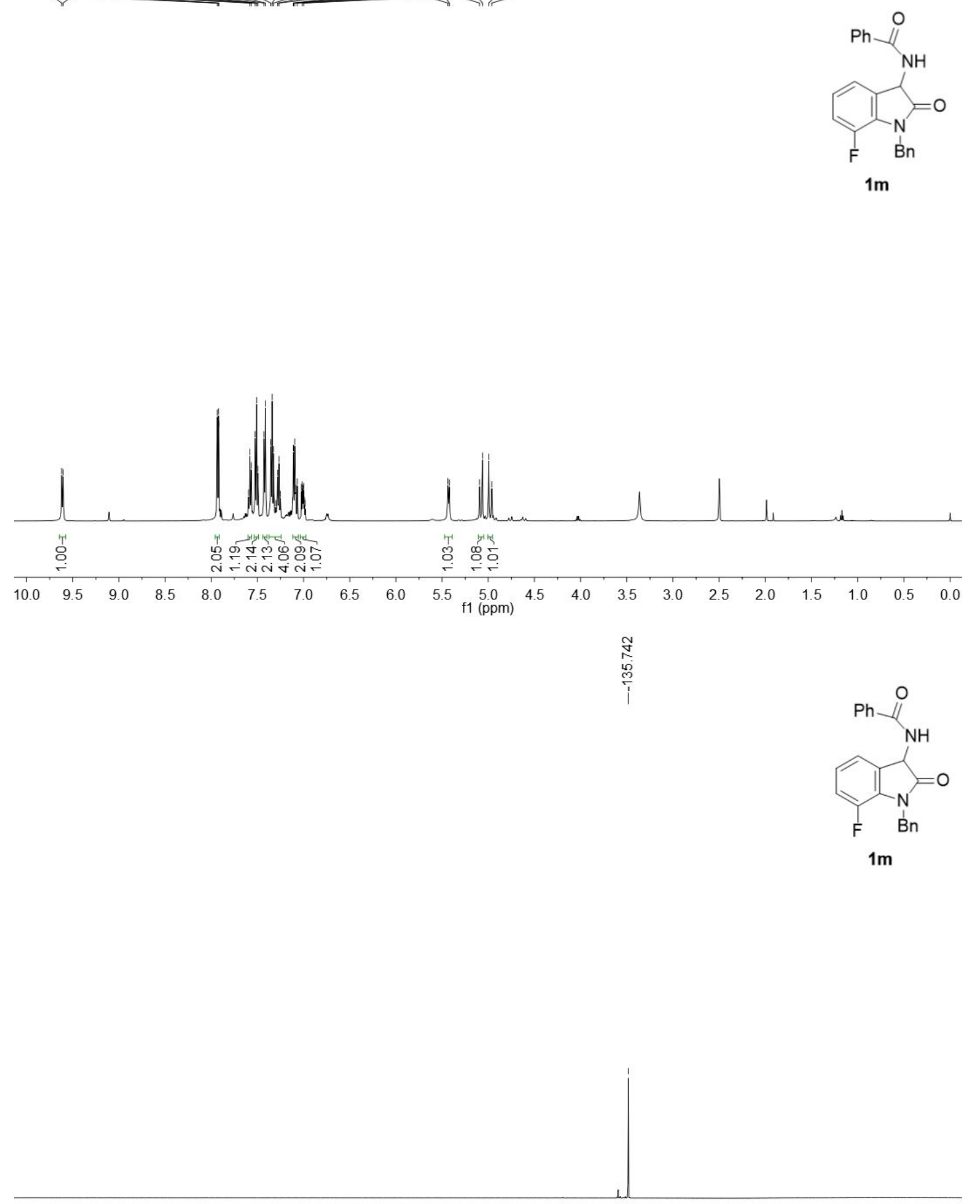

\begin{tabular}{lllllllllllllllllll}
\hline 0 & 10 & 0 & -10 & -20 & -30 & -40 & -50 & -60 & -70 & -80 & -90 & & -110 & -130 & -150 & -170 & -190 & -210
\end{tabular} 


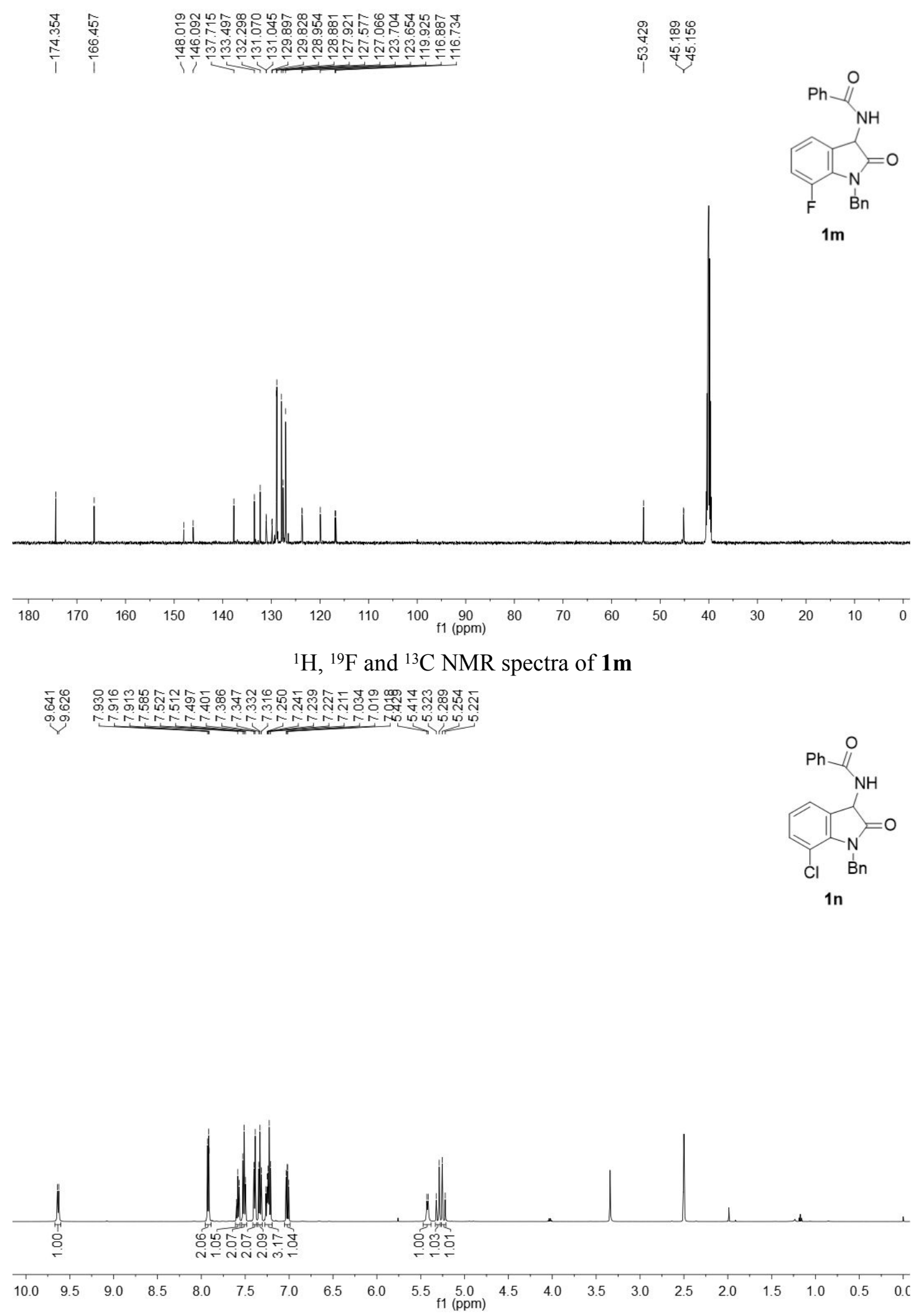




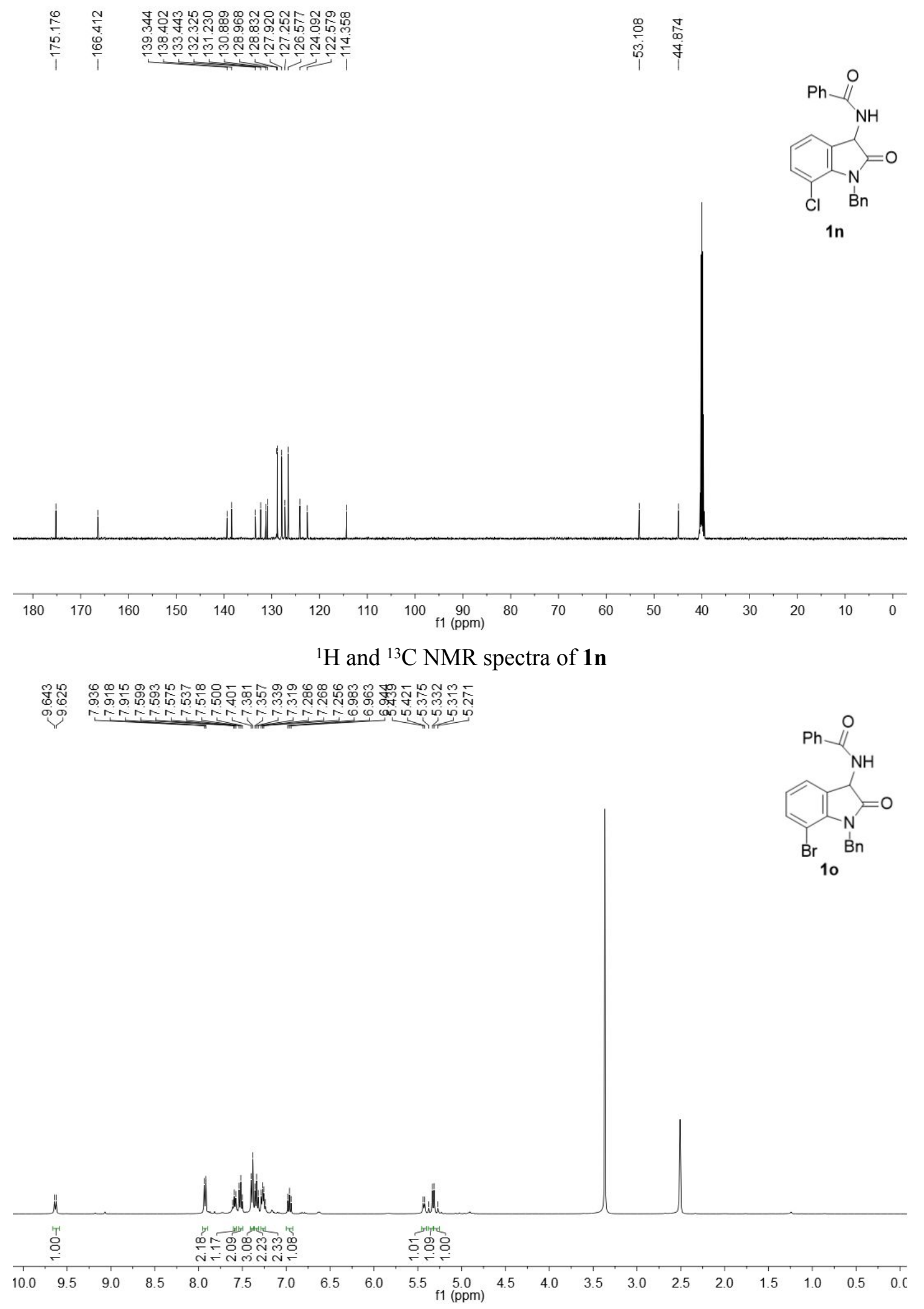




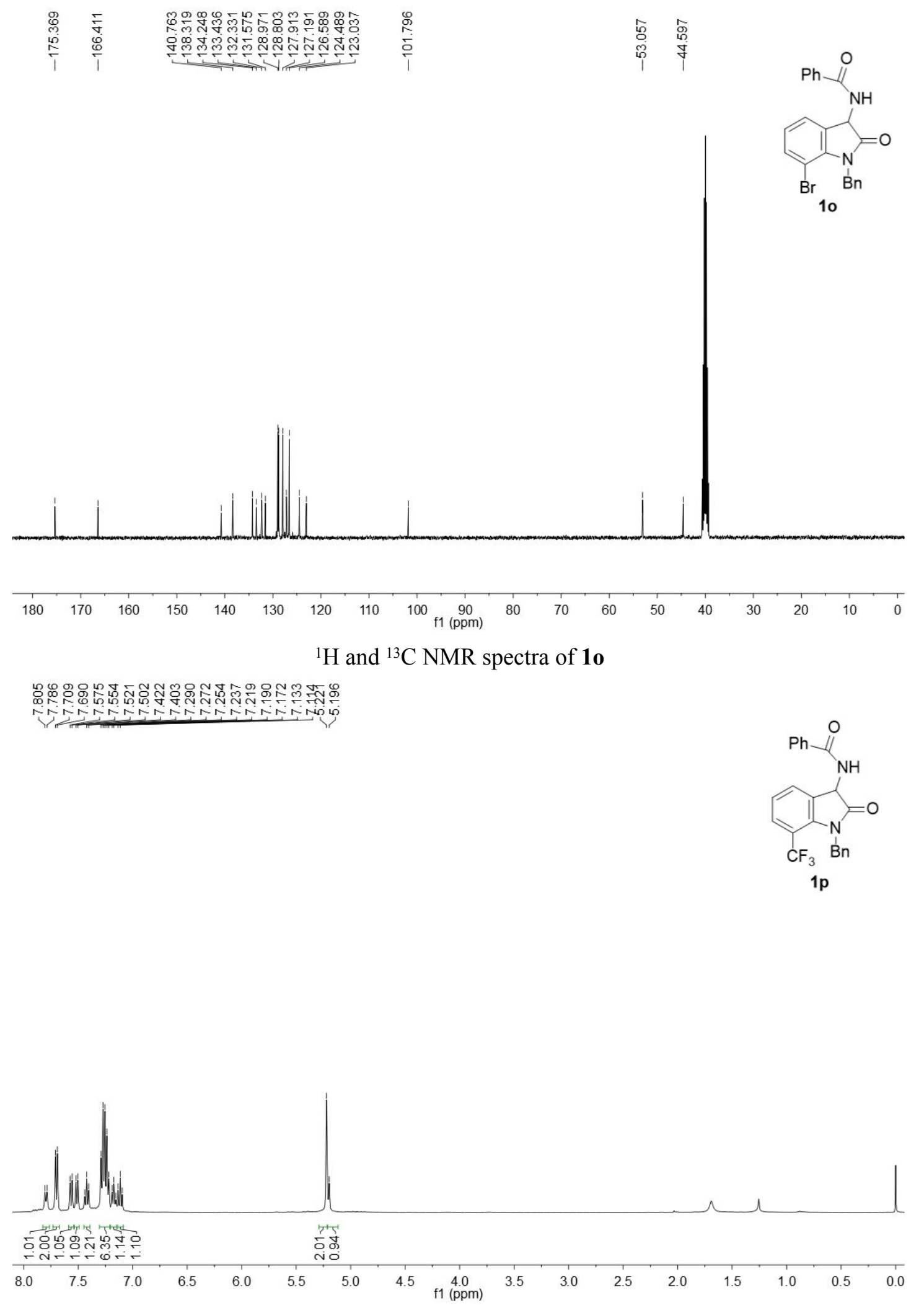



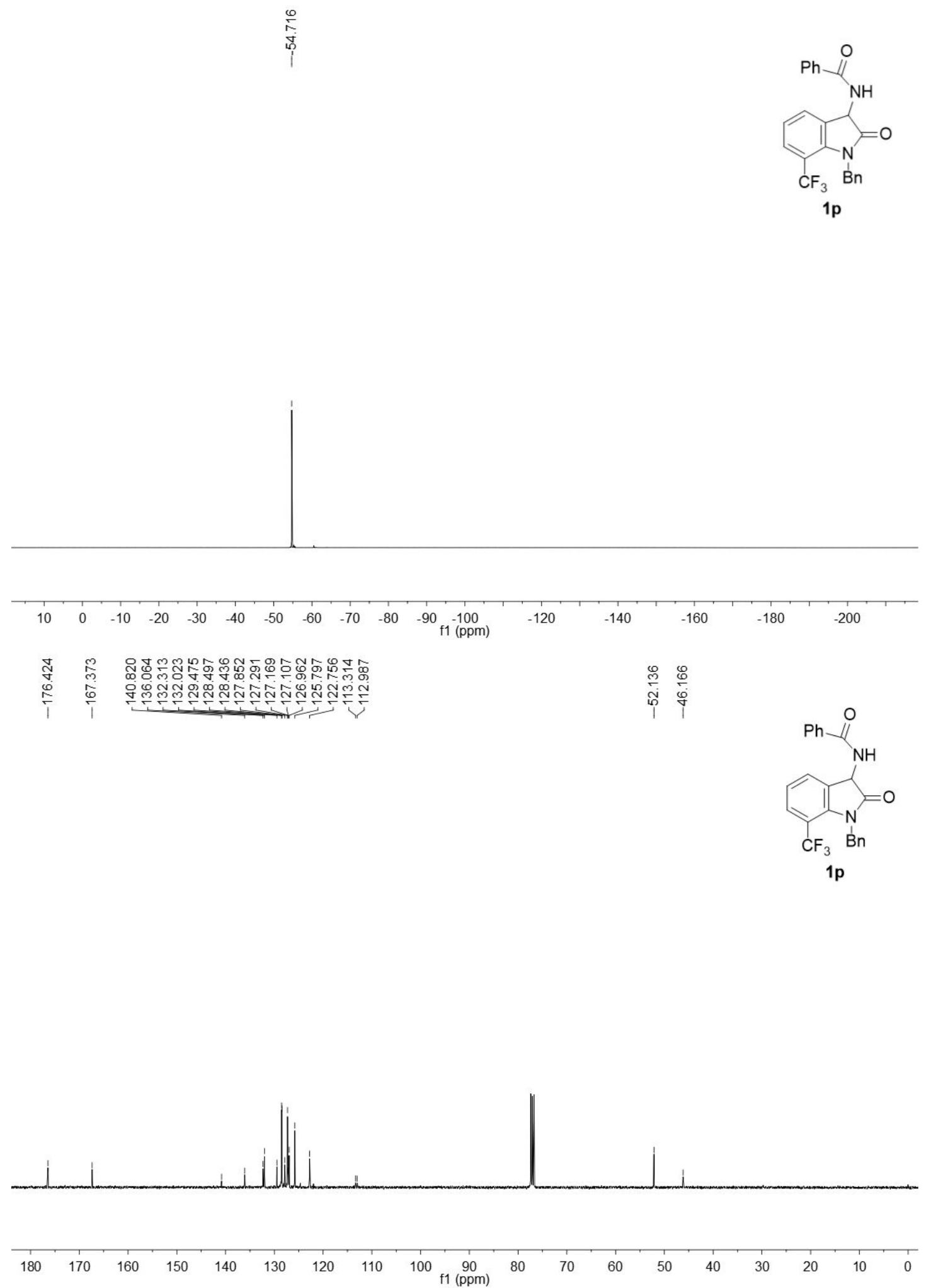

${ }^{1} \mathrm{H},{ }^{19} \mathrm{~F}$ and ${ }^{13} \mathrm{C}$ NMR spectra of $\mathbf{1 p}$ 


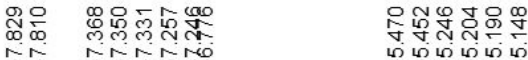

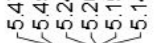

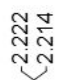
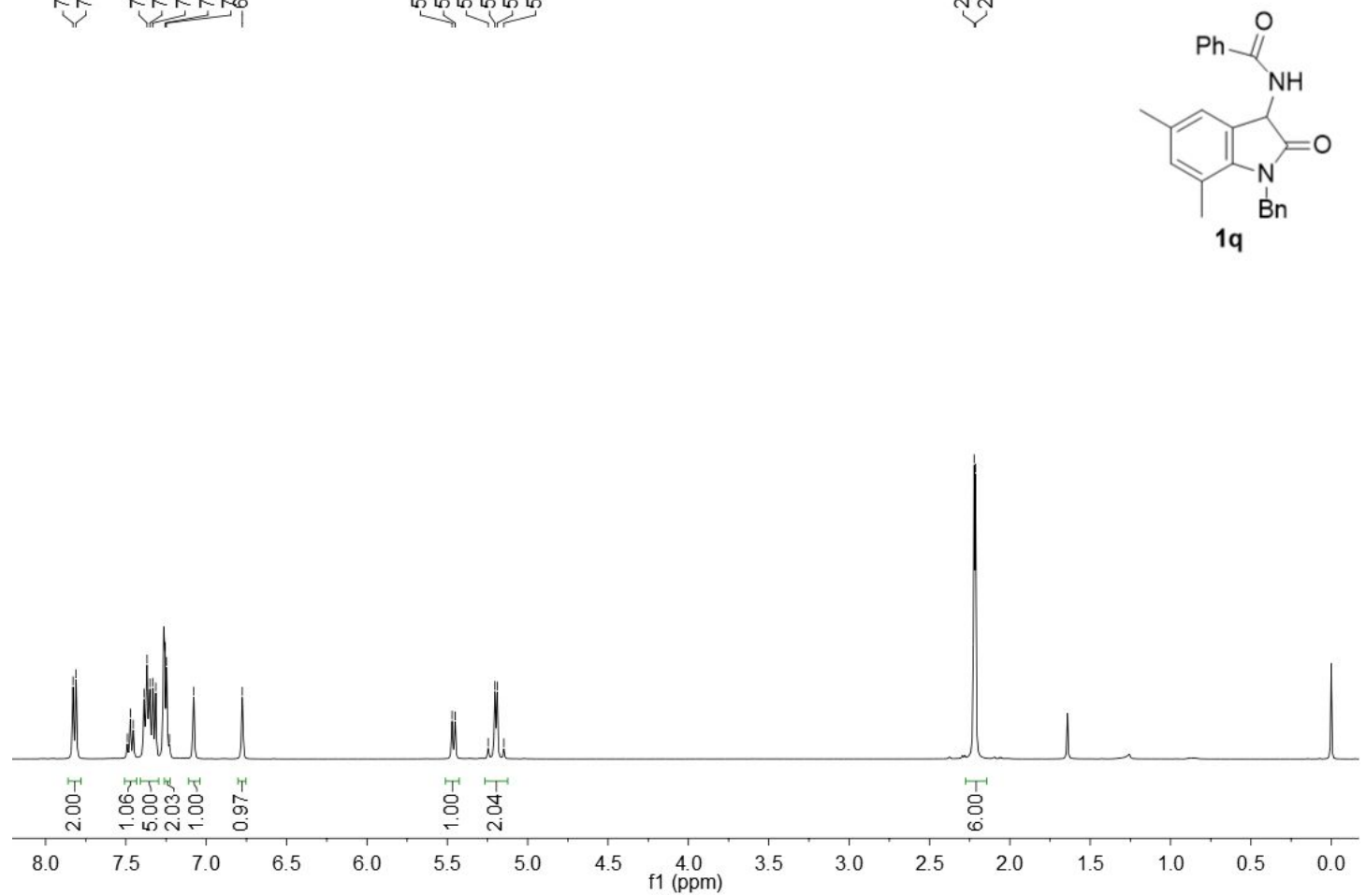

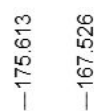

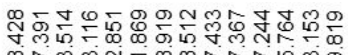

ס.

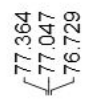

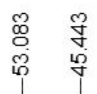

ஸิ)
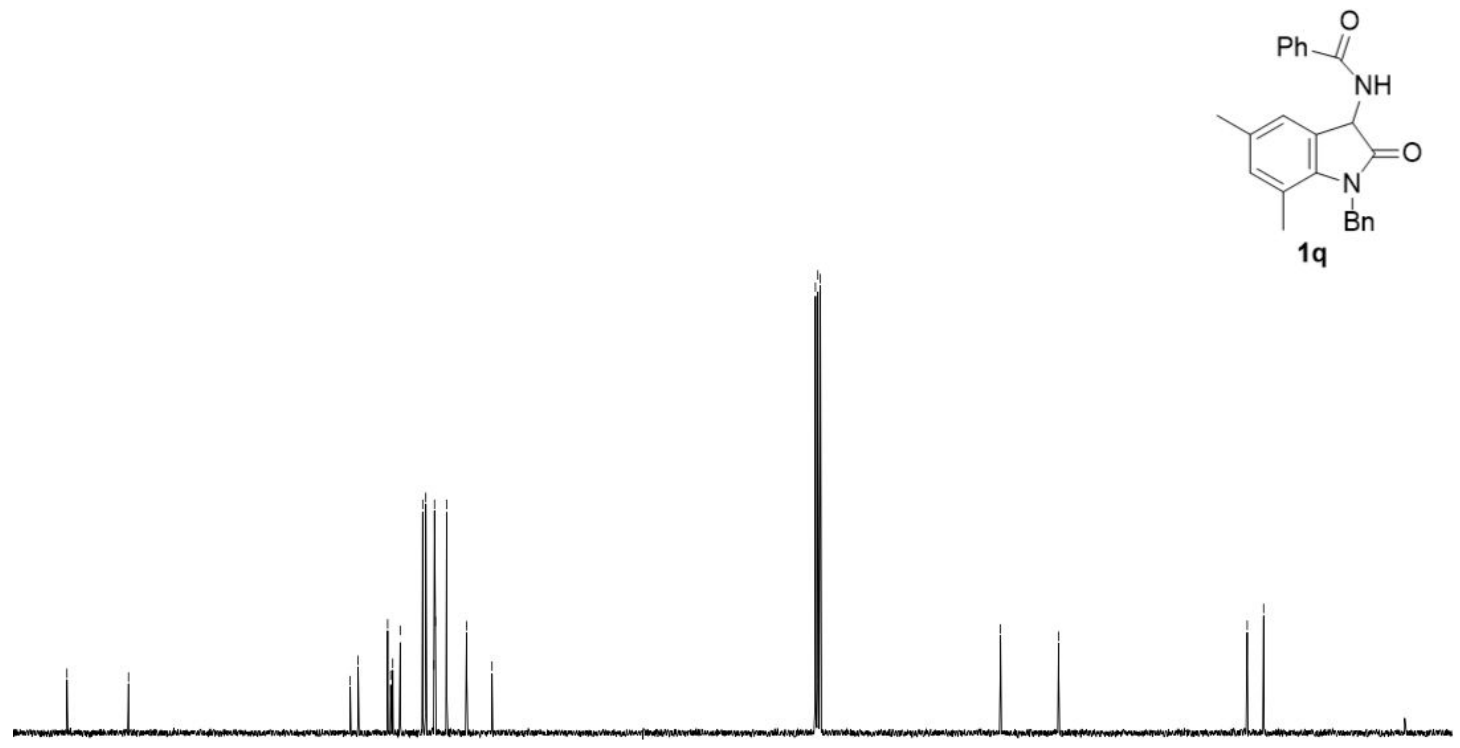

180

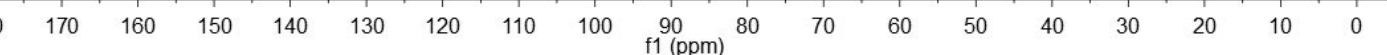

${ }^{1} \mathrm{H}$ and ${ }^{13} \mathrm{C}$ NMR spectra of $\mathbf{1 q}$ 
$\begin{array}{llll}0 & 0 \\ 0 & 0\end{array}$
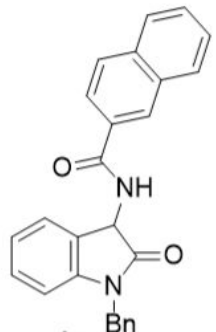

1r $\mathrm{Bn}$

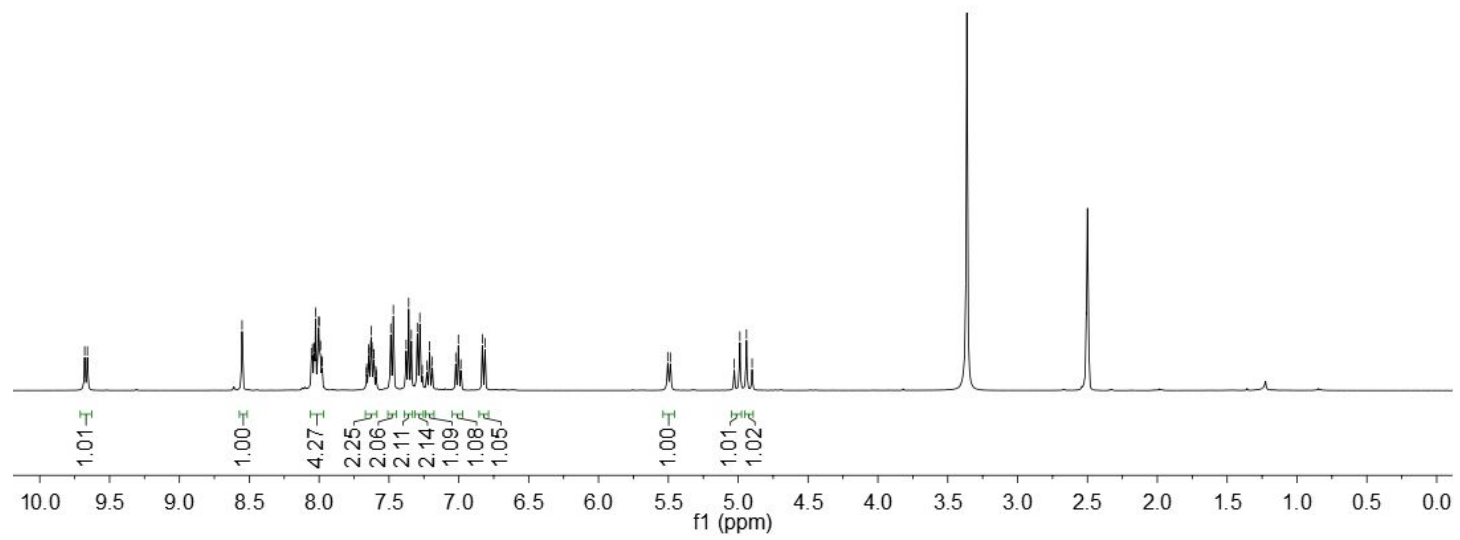

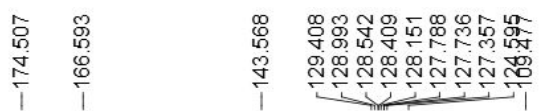

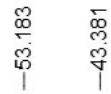
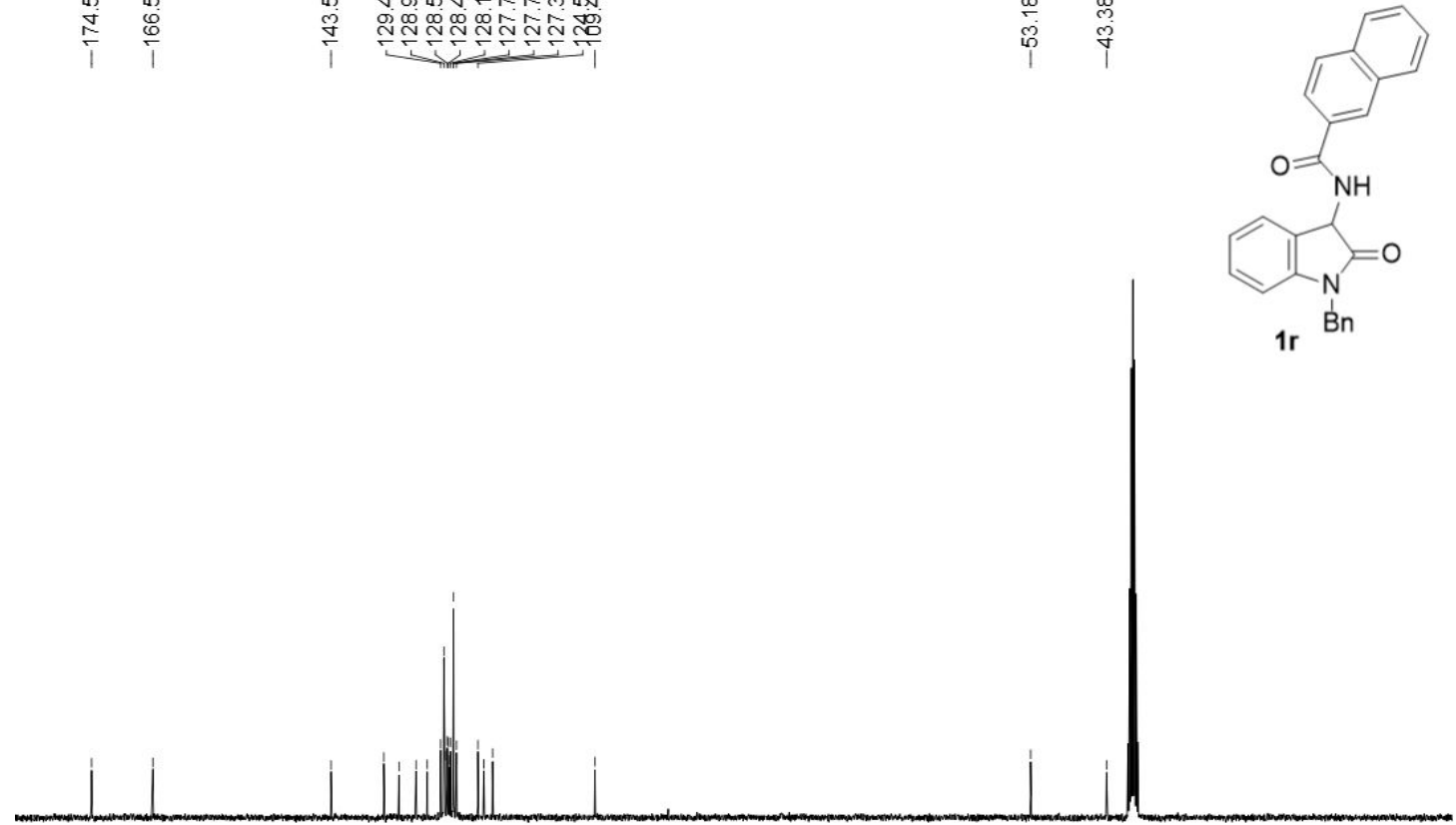

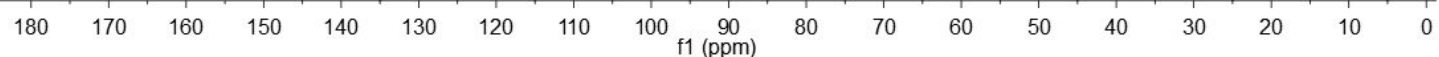

${ }^{1} \mathrm{H}$ and ${ }^{13} \mathrm{C}$ NMR spectra of $\mathbf{1 r}$ 

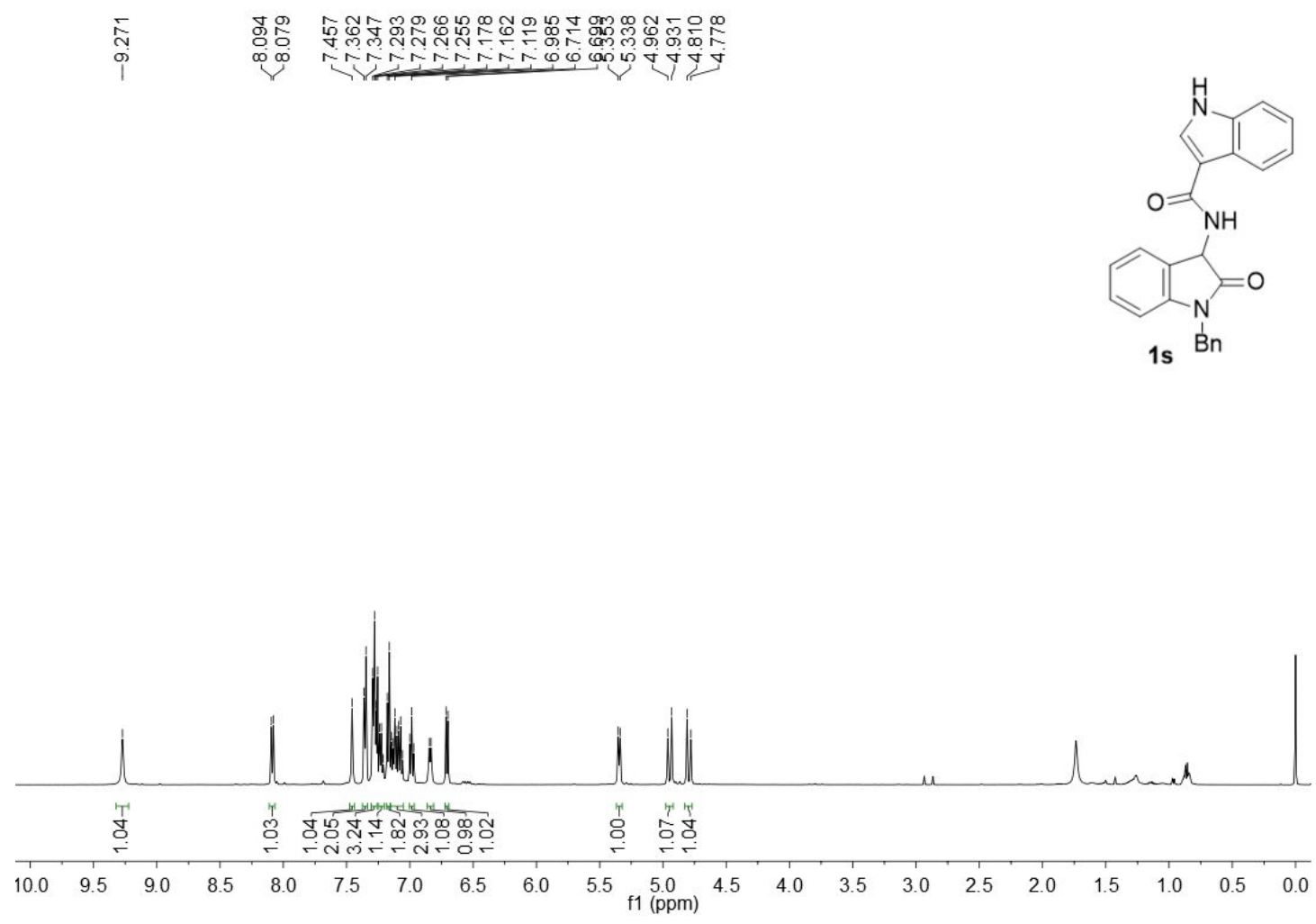

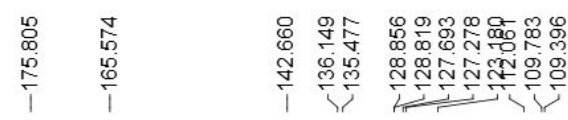

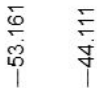
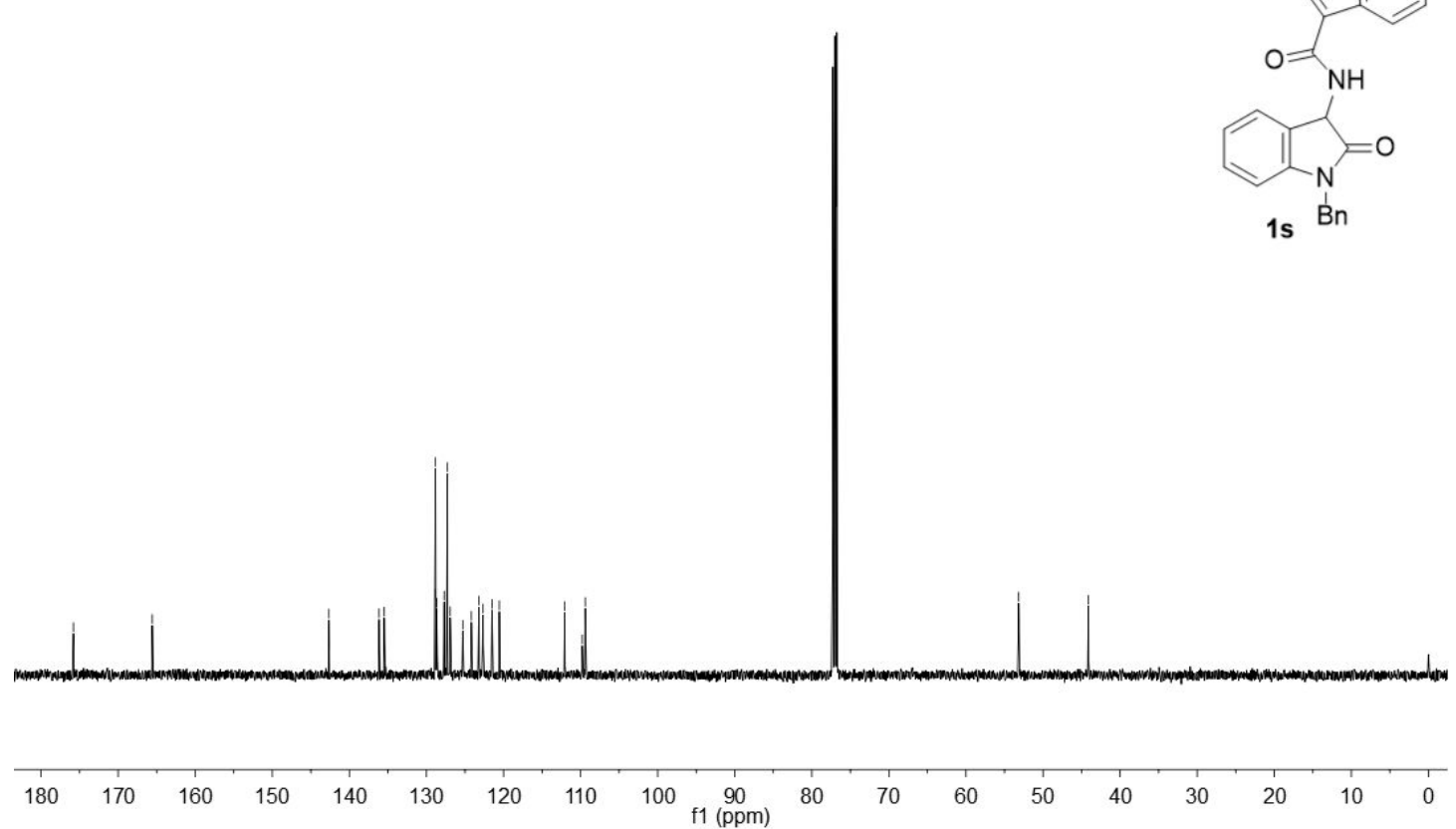

${ }^{1} \mathrm{H}$ and ${ }^{13} \mathrm{C}$ NMR spectra of $\mathbf{1 s}$ 

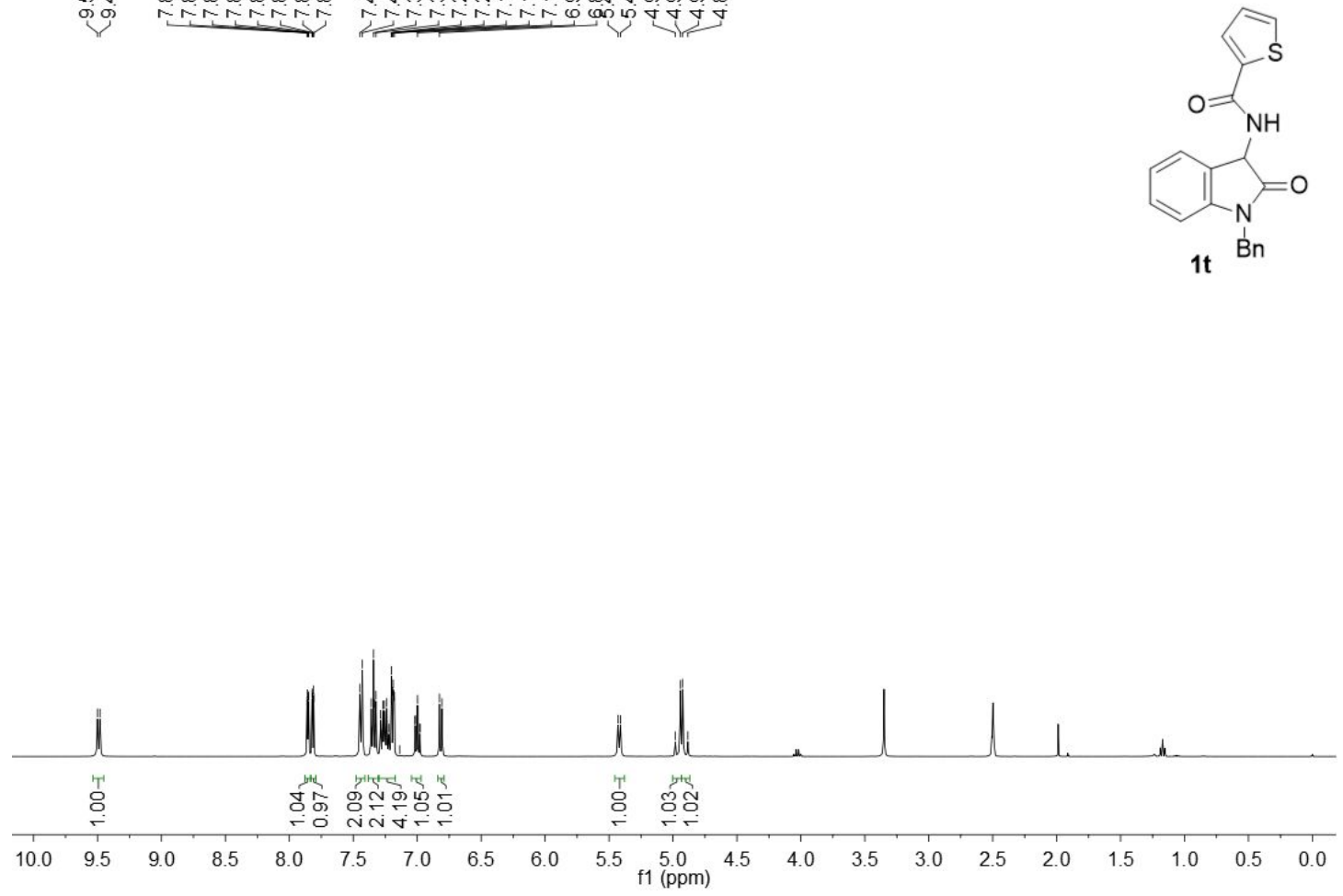

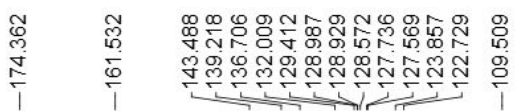
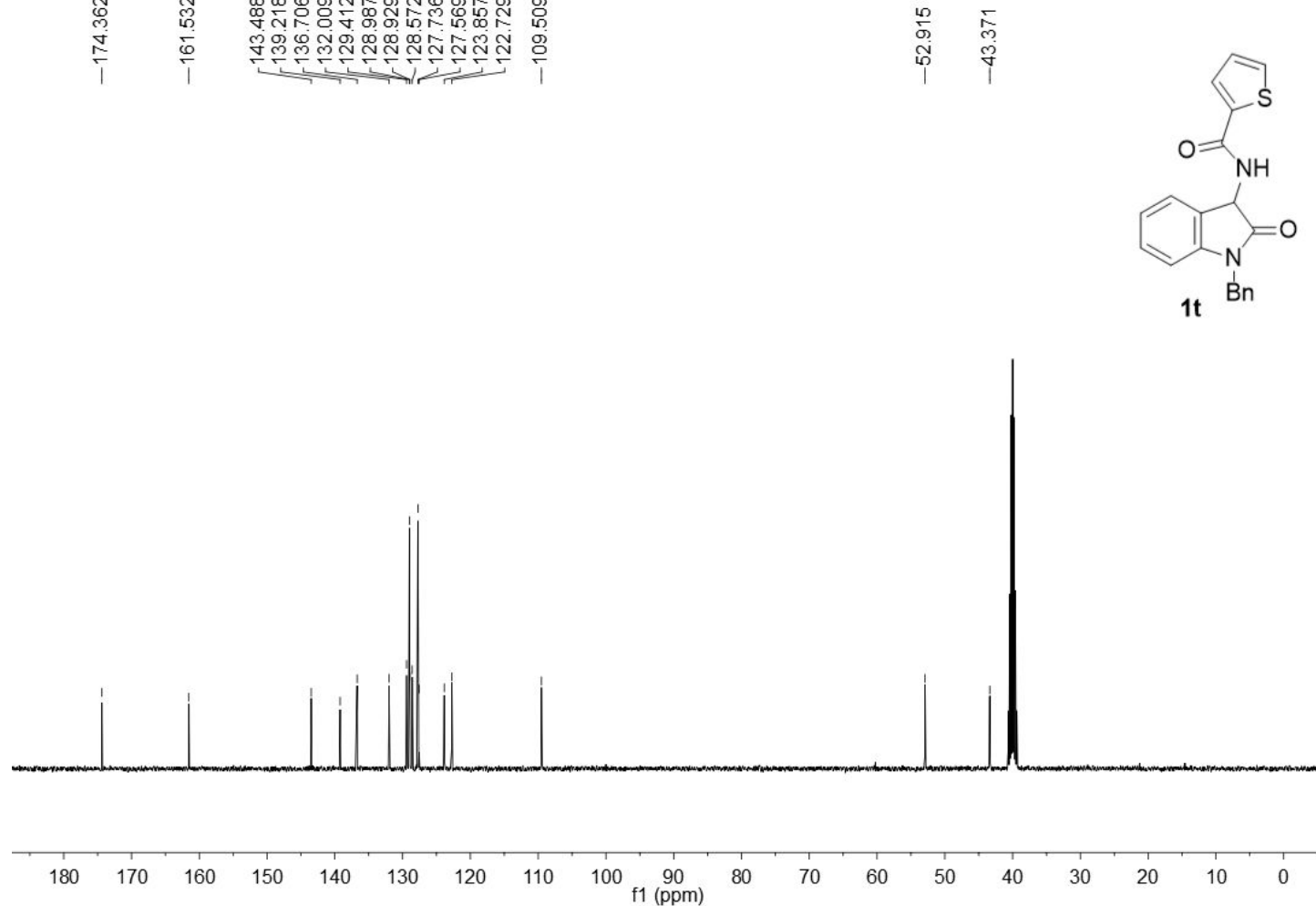

${ }^{1} \mathrm{H}$ and ${ }^{13} \mathrm{C}$ NMR spectra of $\mathbf{1 t}$ 


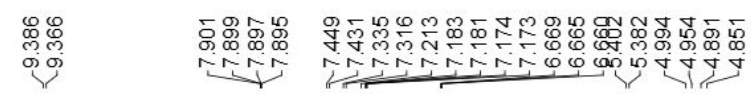

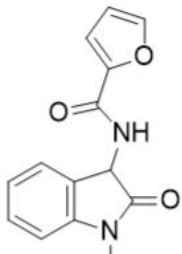

$1 \mathrm{u}^{\mathrm{Bn}}$

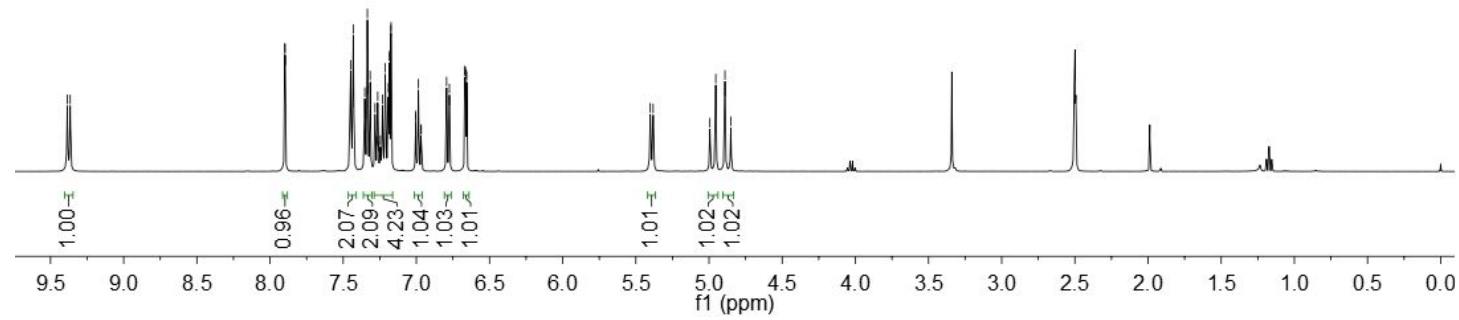

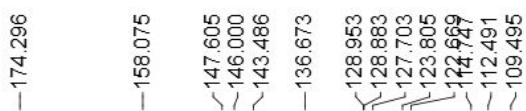

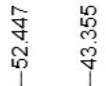

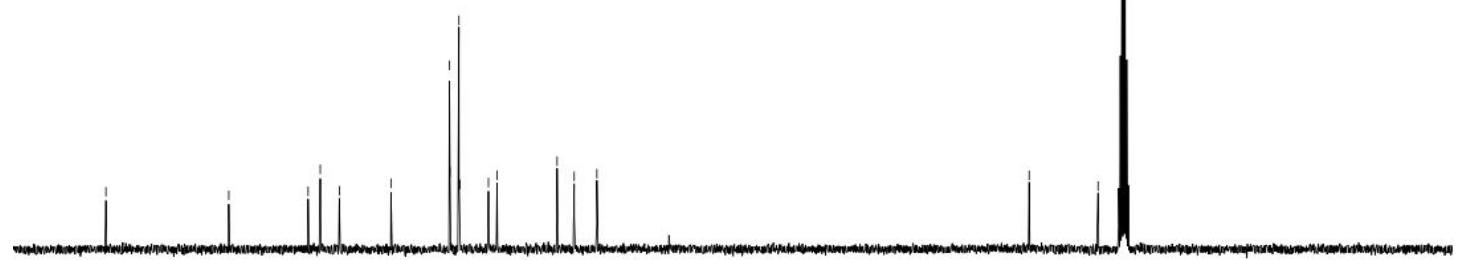

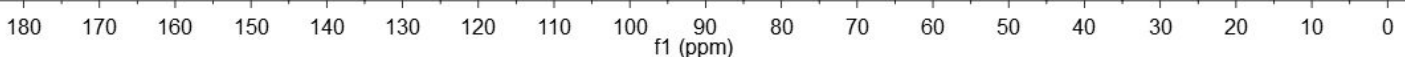

${ }^{1} \mathrm{H}$ and ${ }^{13} \mathrm{C}$ NMR spectra of $\mathbf{1 u}$ 


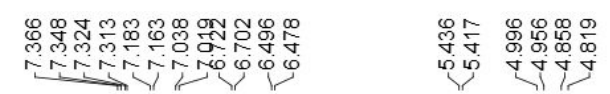

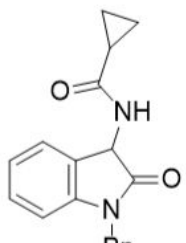

1v $\mathrm{Bn}$

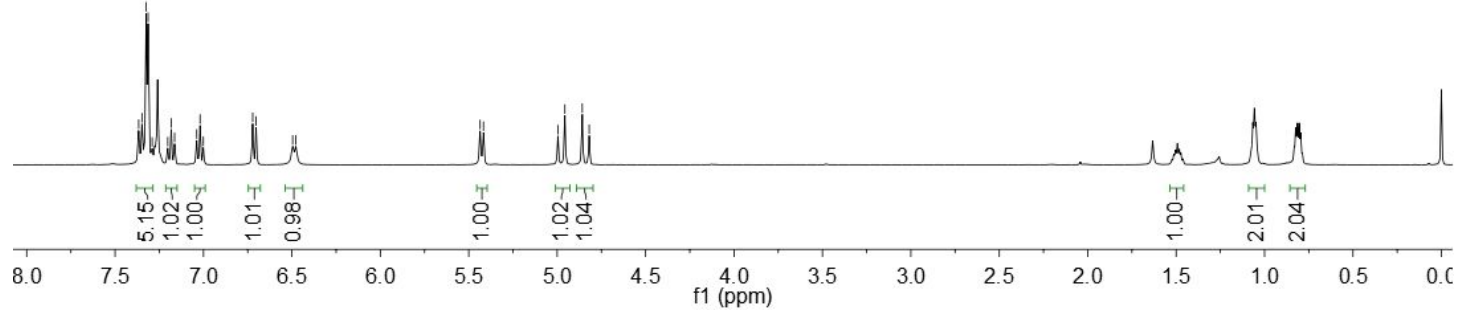

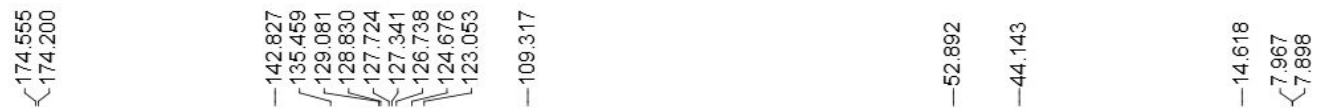

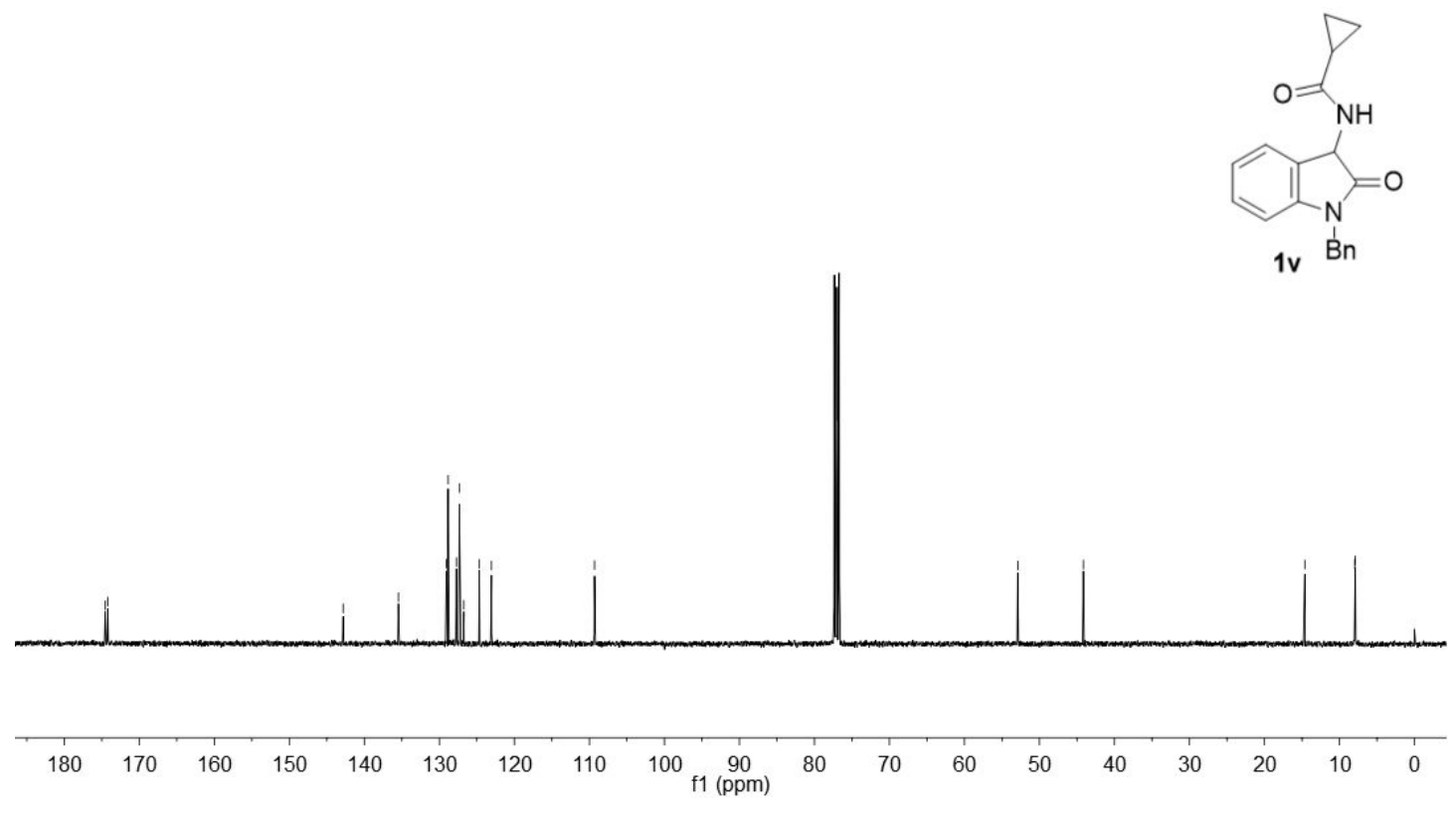

${ }^{1} \mathrm{H}$ and ${ }^{13} \mathrm{C}$ NMR spectra of $\mathbf{1 v}$ 

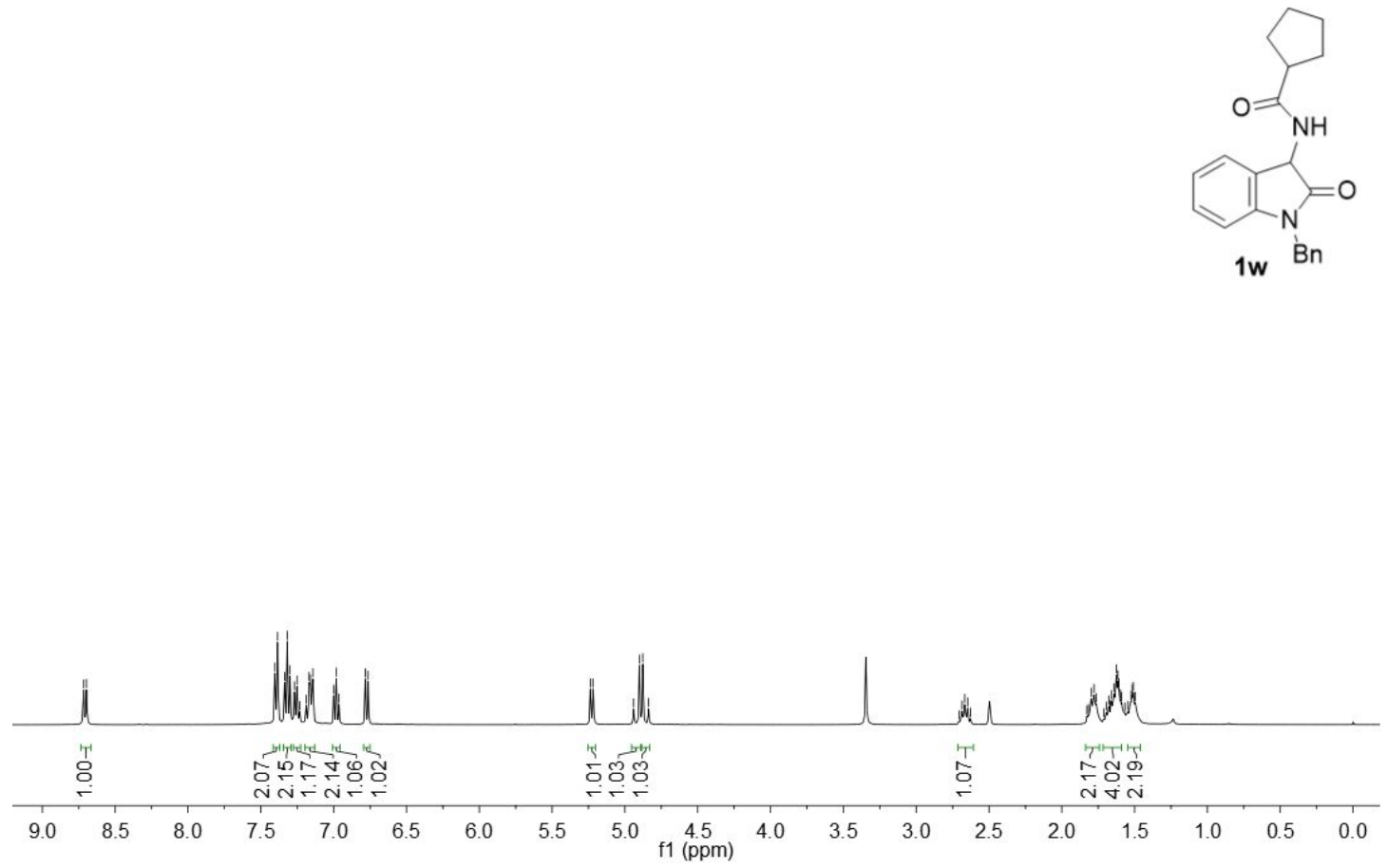

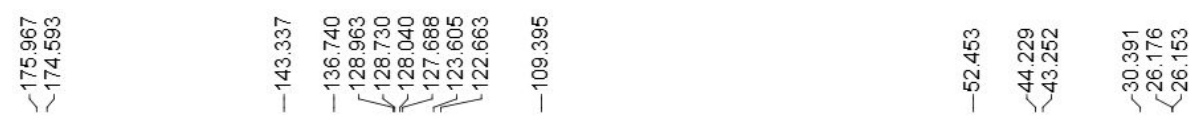
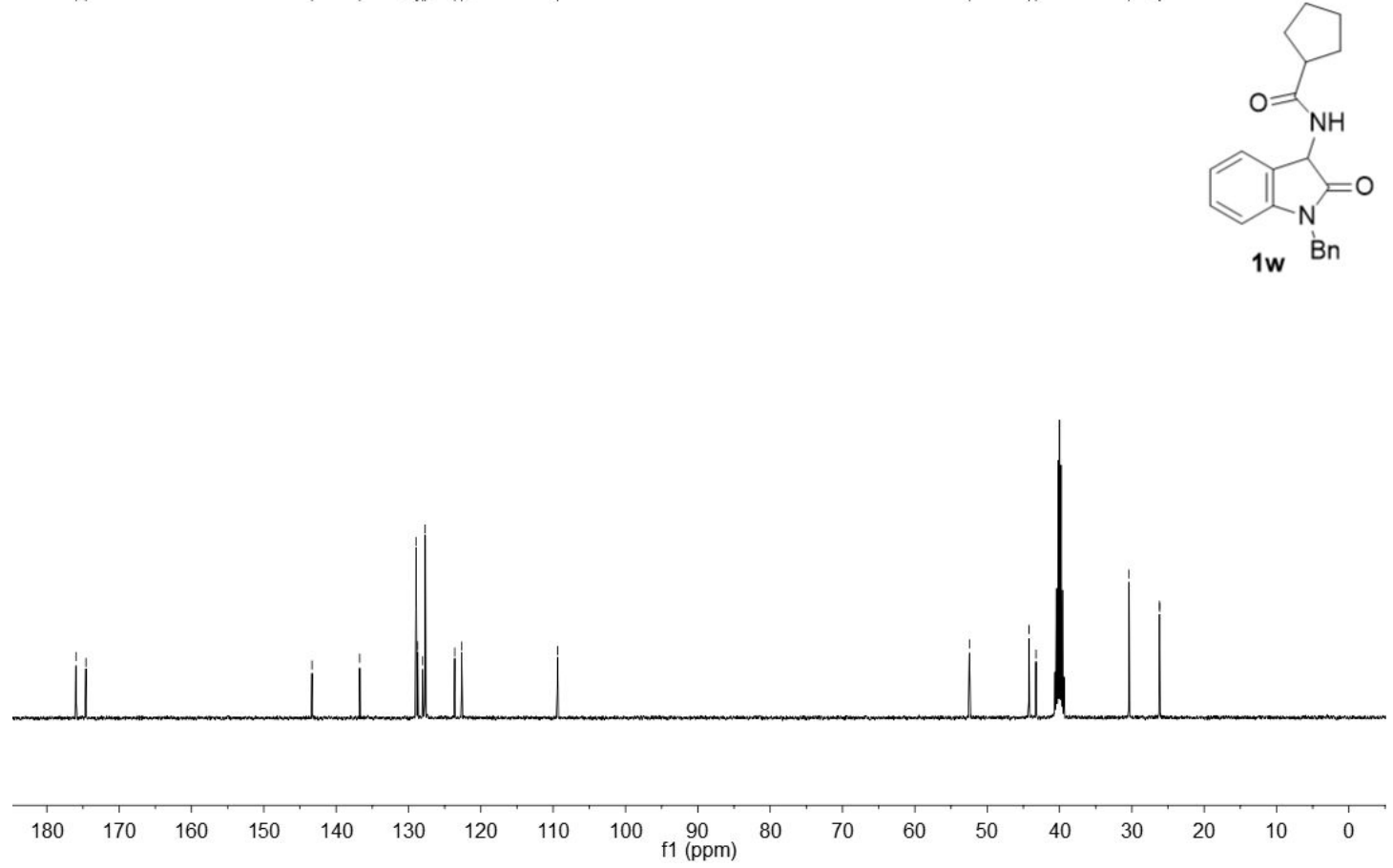

${ }^{1} \mathrm{H}$ and ${ }^{13} \mathrm{C}$ NMR spectra of $\mathbf{1 w}$ 


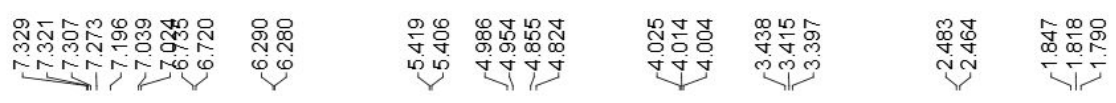
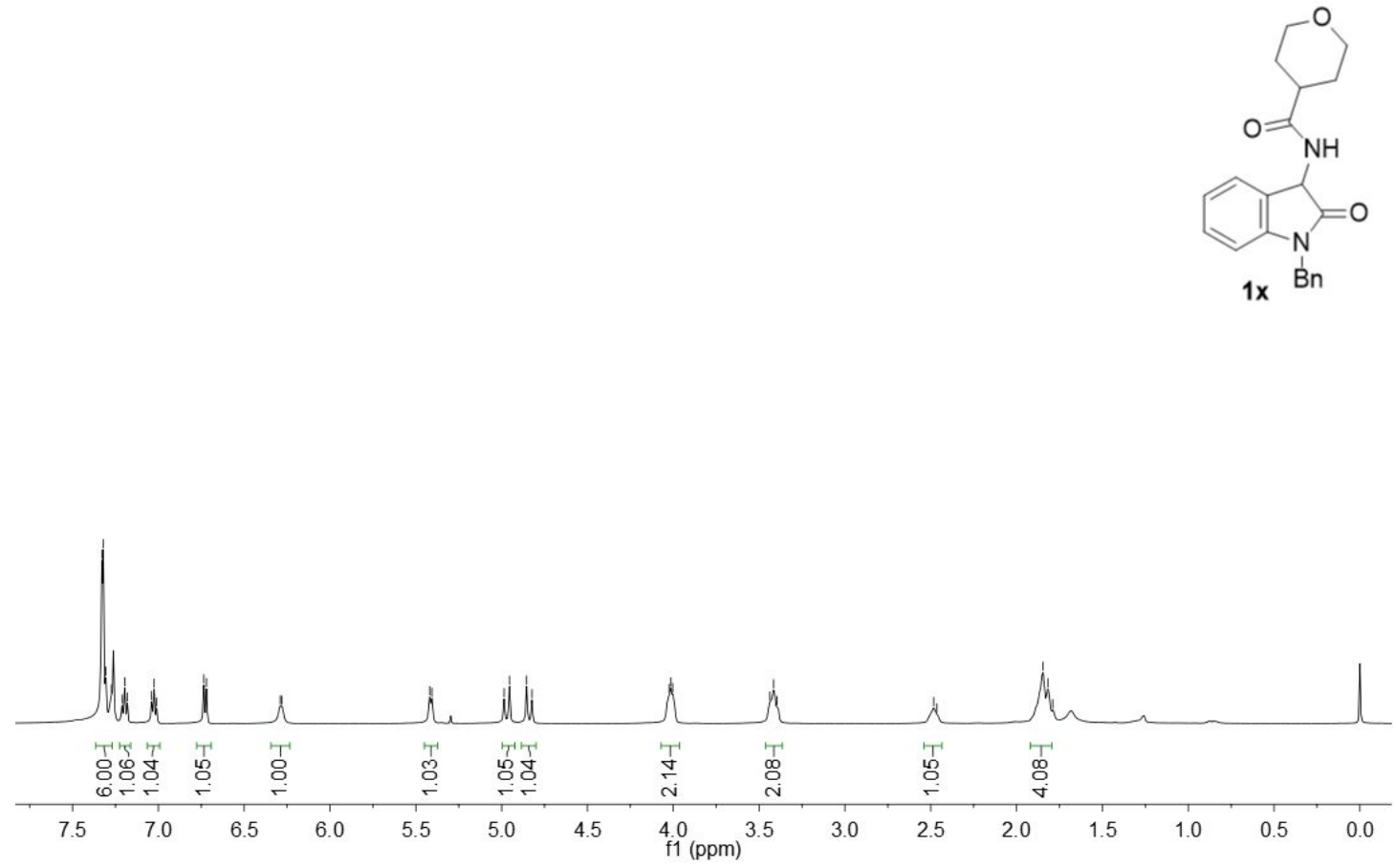

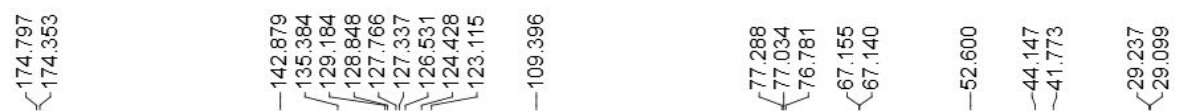

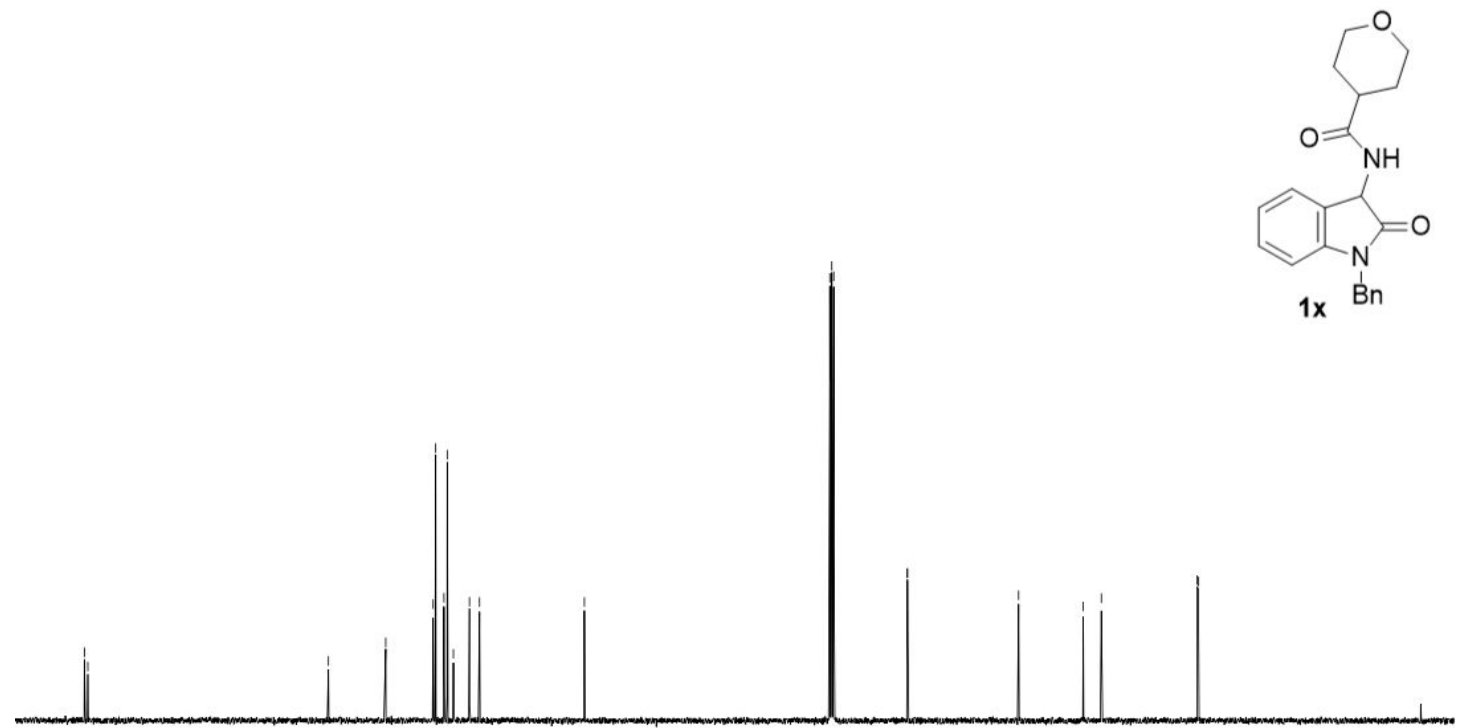

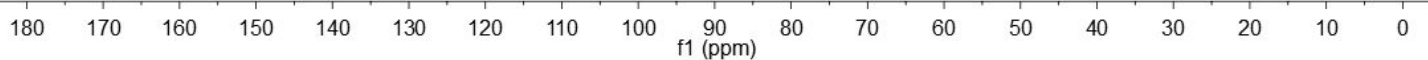

${ }^{1} \mathrm{H}$ and ${ }^{13} \mathrm{C}$ NMR spectra of $\mathbf{1} \mathbf{x}$ 

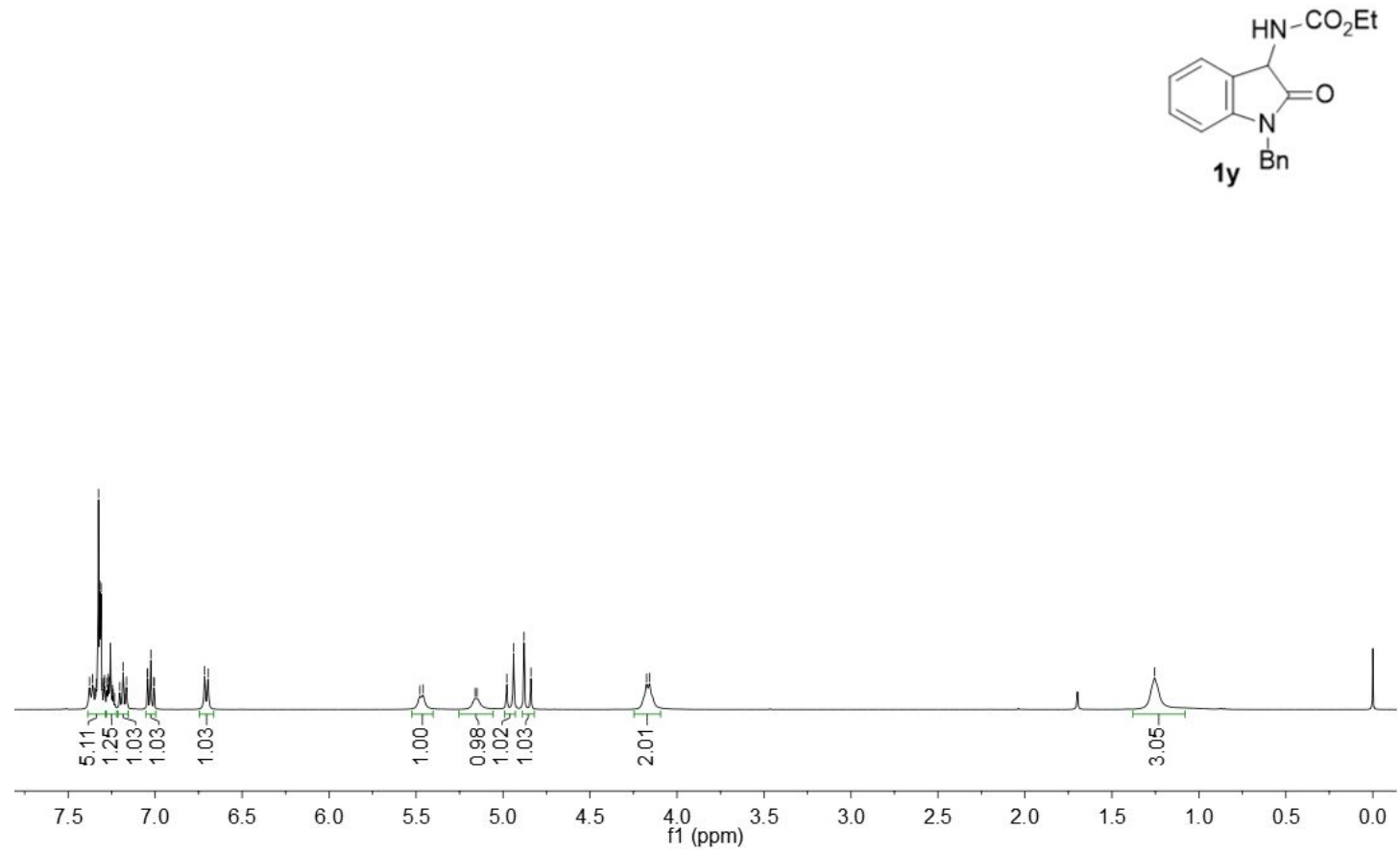

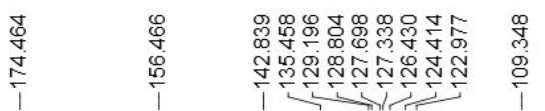

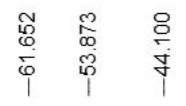

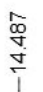

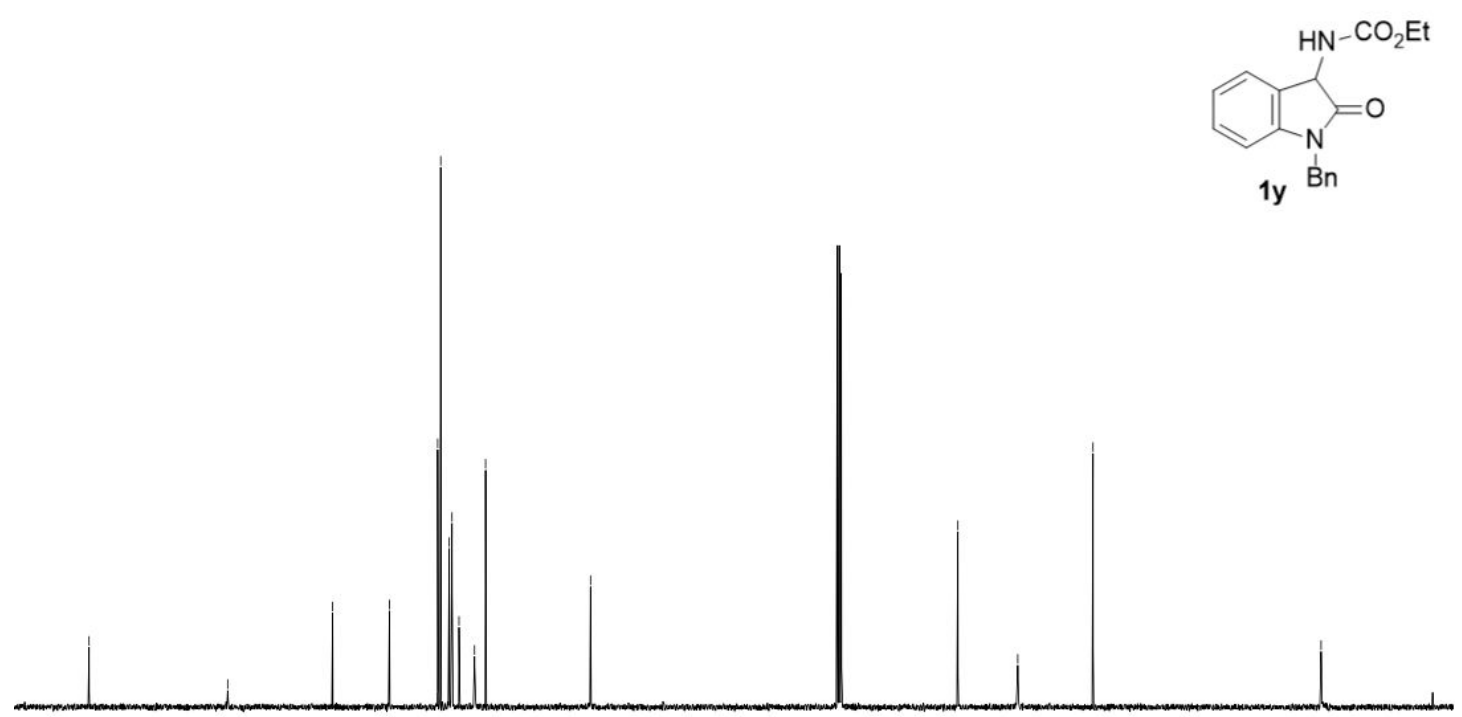

$\begin{array}{lllllllllllllllllll}180 & 170 & 160 & 150 & 140 & 130 & 120 & 110 & 100 & \begin{array}{c}90 \\ \mathrm{f} 1(\mathrm{ppm})\end{array} & 80 & 70 & 60 & 50 & 40 & 30 & 20 & 10 & 0\end{array}$

${ }^{1} \mathrm{H}$ and ${ }^{13} \mathrm{C}$ NMR spectra of $\mathbf{1 y}$ 


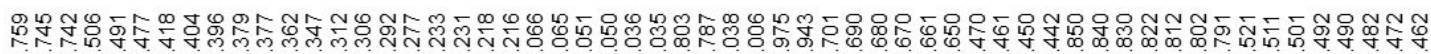

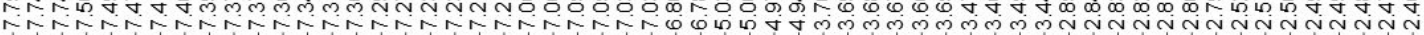

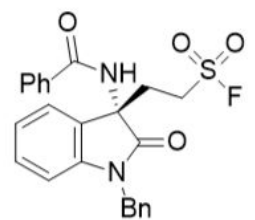

$3 a$

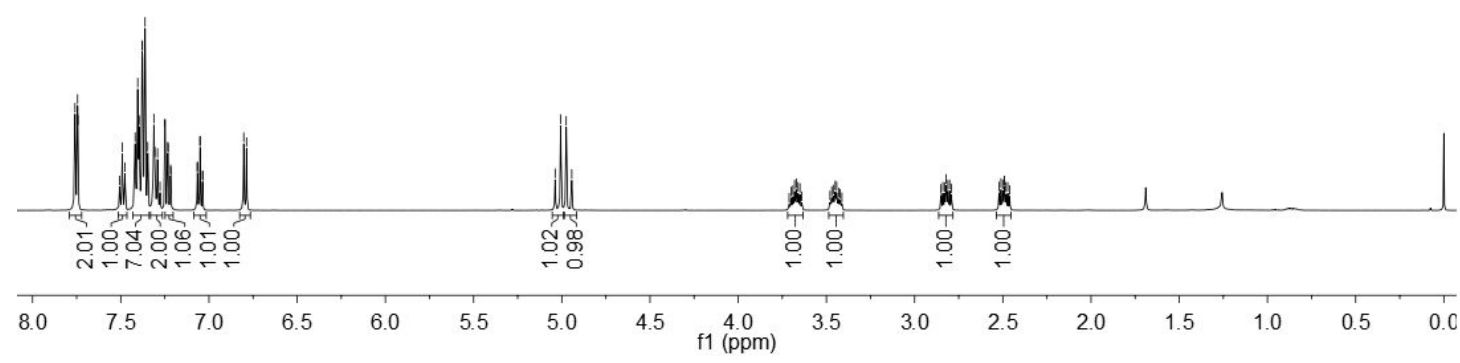

ֻ

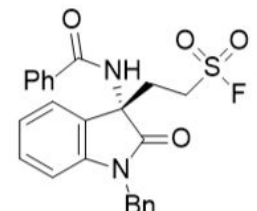

$3 a$

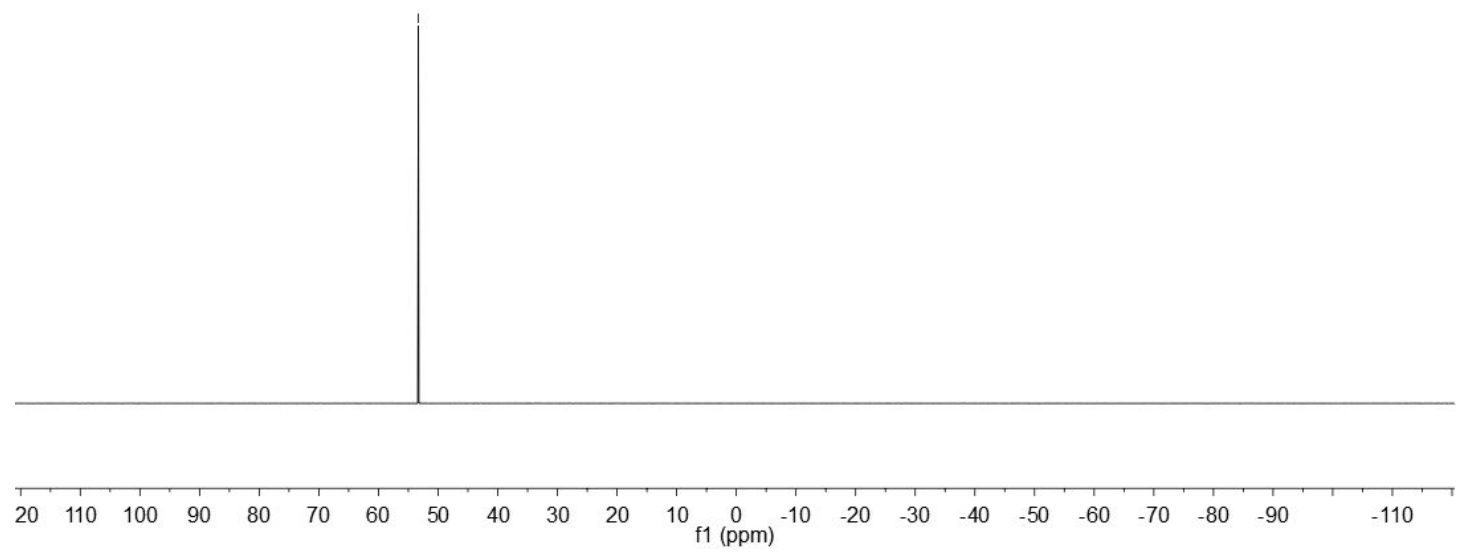




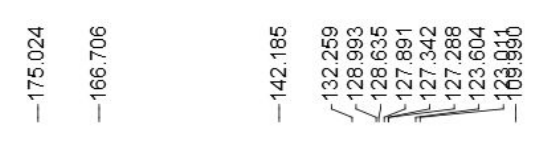

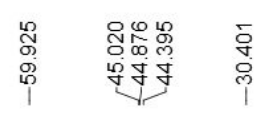

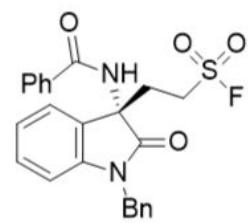

3 a

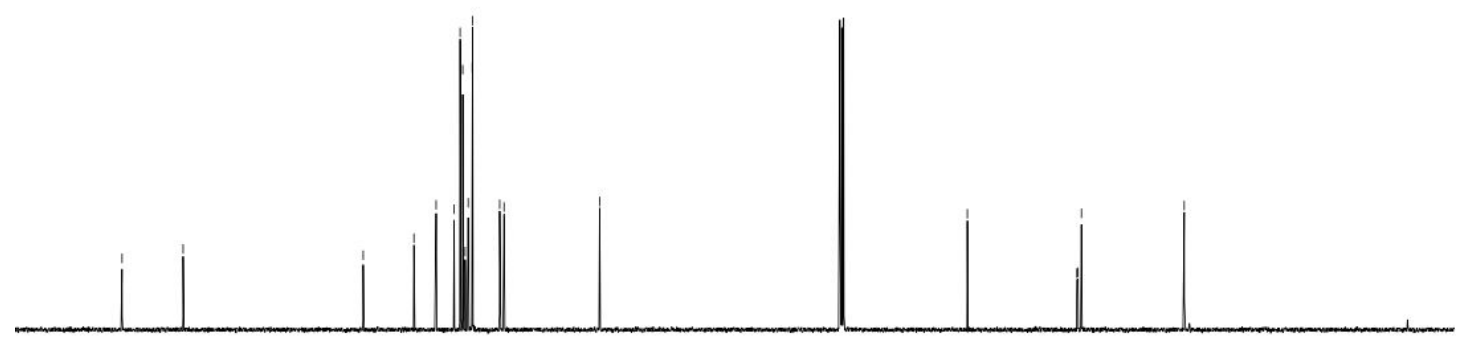

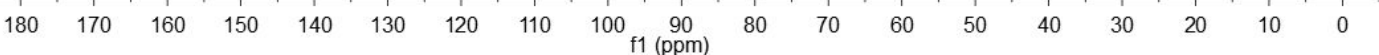

${ }^{1} \mathrm{H},{ }^{19} \mathrm{~F}$ and ${ }^{13} \mathrm{C}$ NMR spectra of $\mathbf{3 a}$

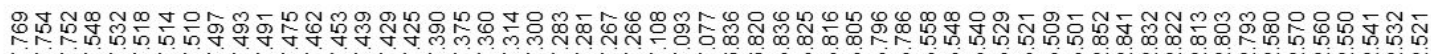

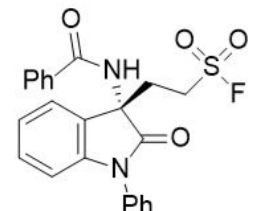

3b

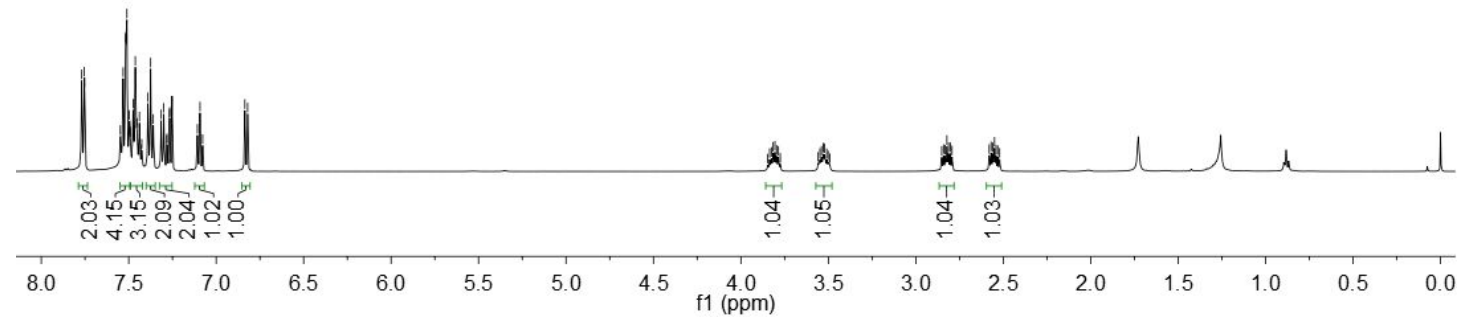




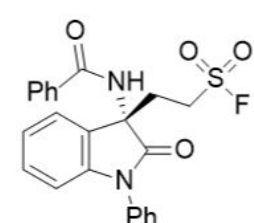

$3 b$

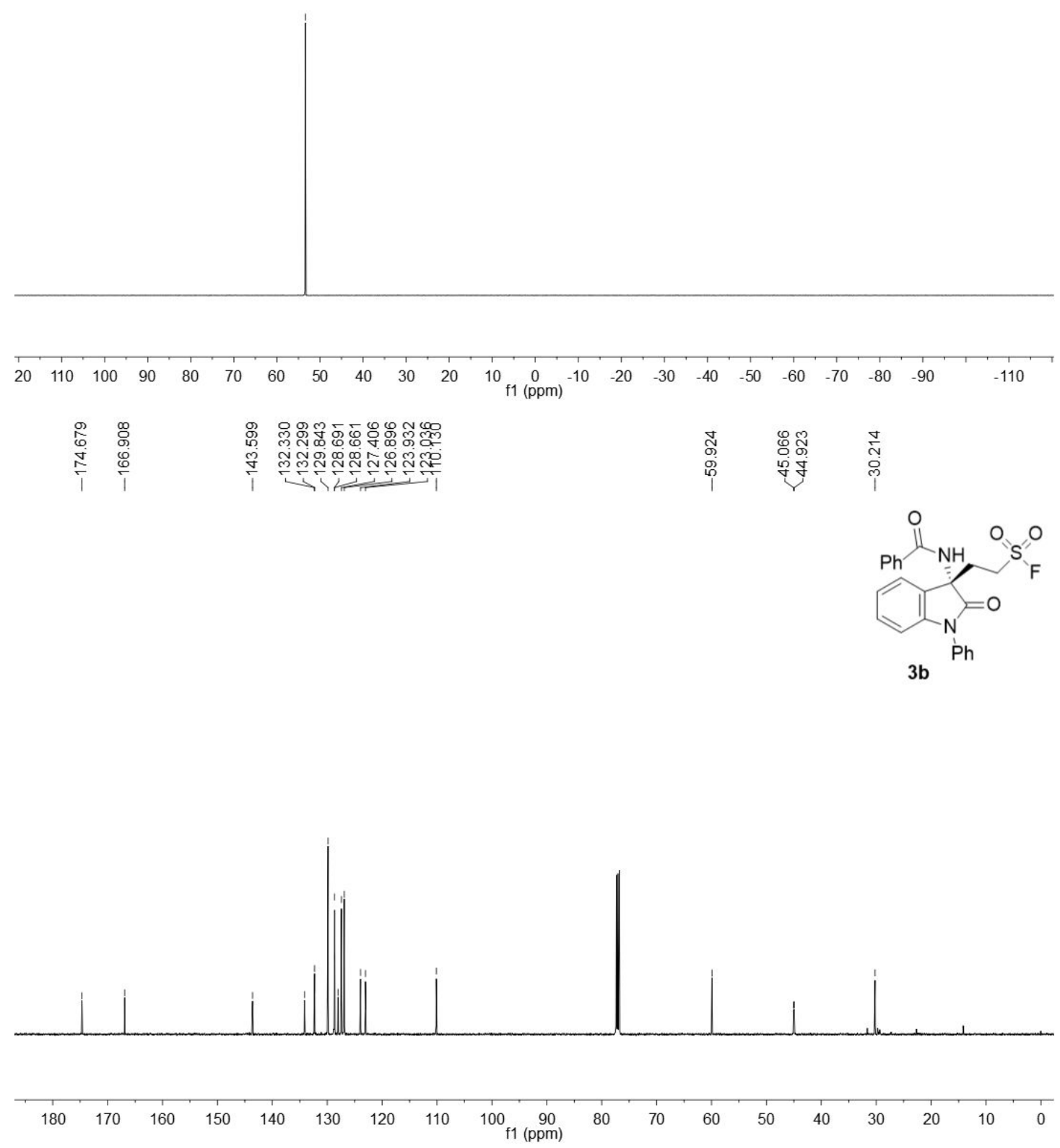

${ }^{1} \mathrm{H},{ }^{19} \mathrm{~F}$ and ${ }^{13} \mathrm{C}$ NMR spectra of $\mathbf{3 b}$ 


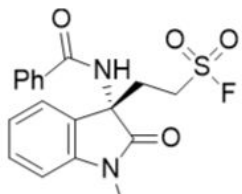

$3 c$

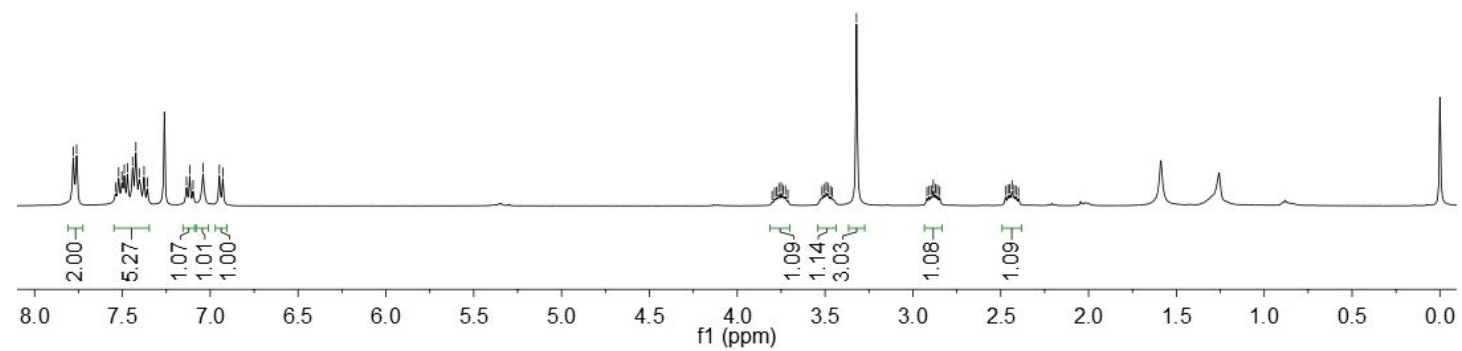

๑
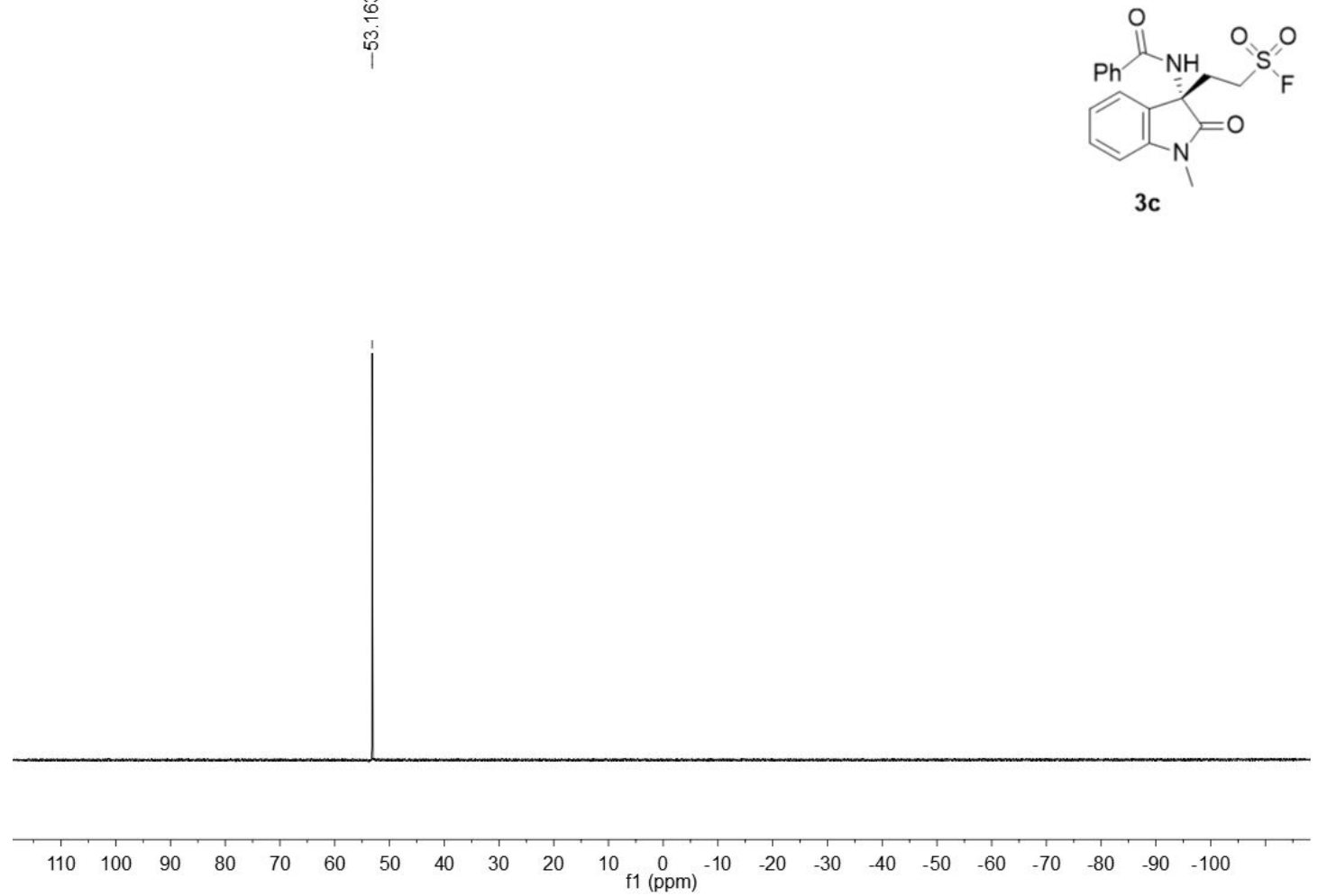


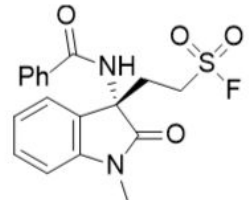

3c
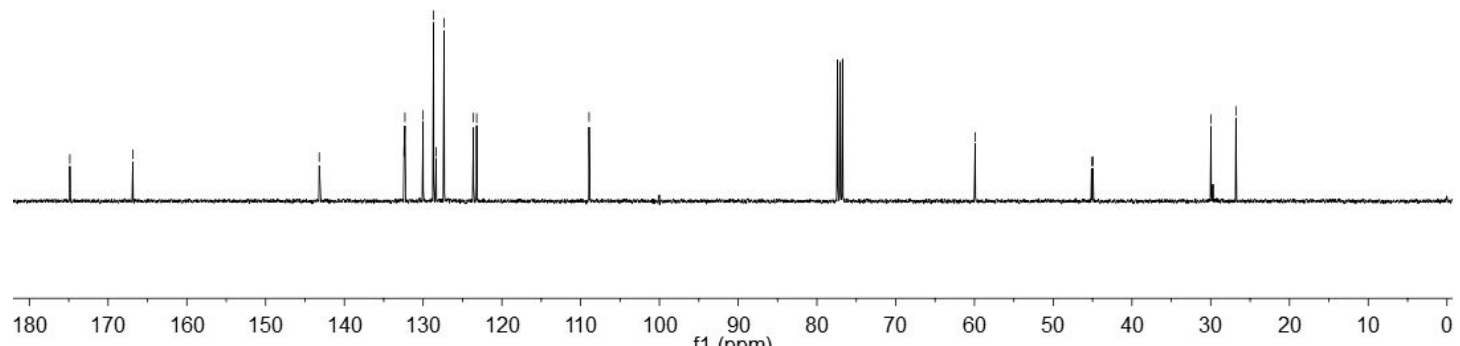

${ }^{1} \mathrm{H},{ }^{19} \mathrm{~F}$ and ${ }^{13} \mathrm{C}$ NMR spectra of $\mathbf{3 c}$

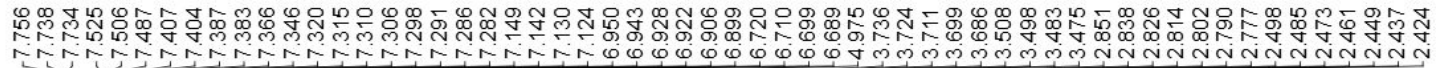

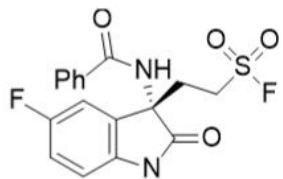

$3 d$

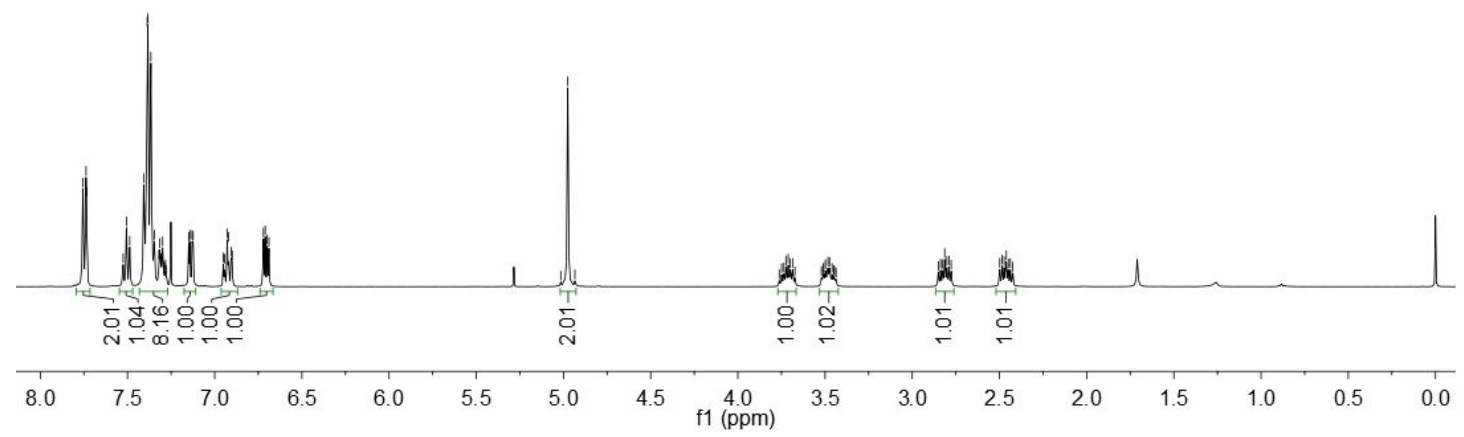


্ֻল

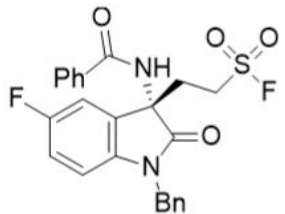

3d

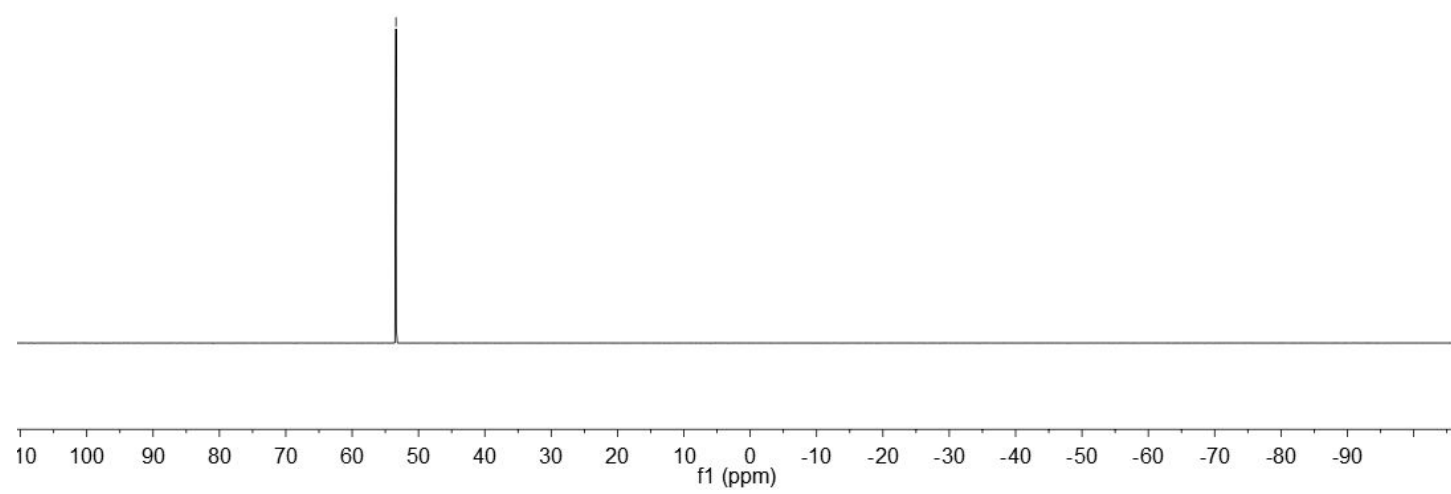

总

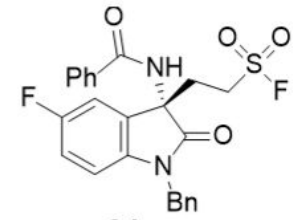

3d

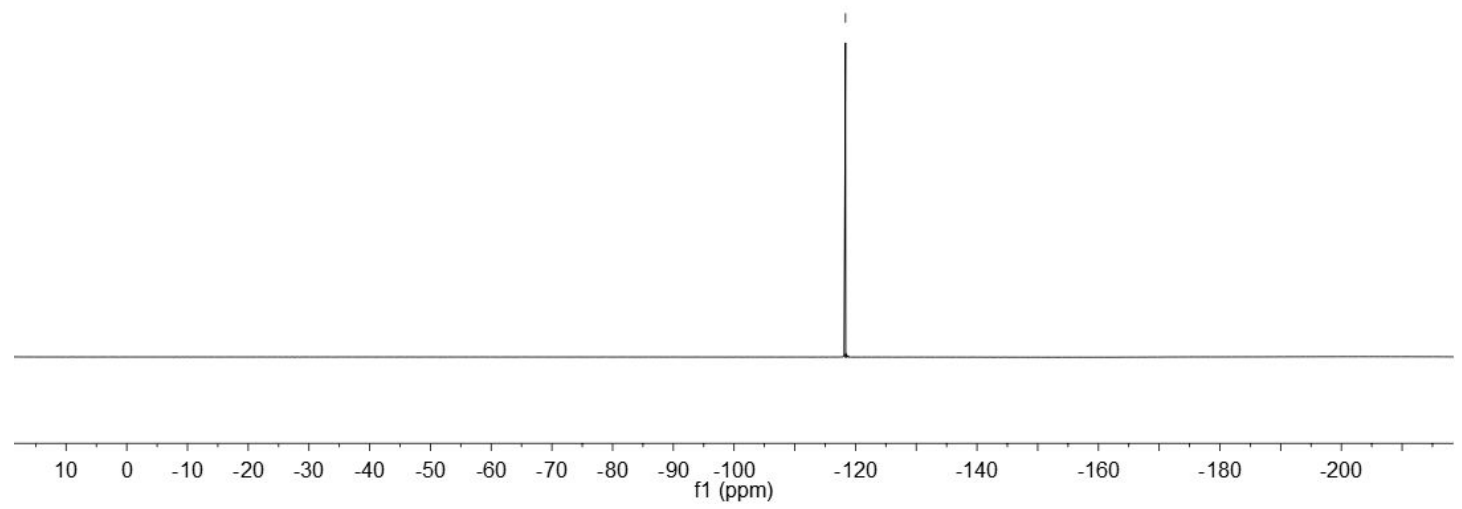




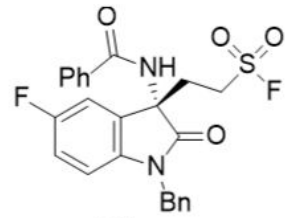

3d

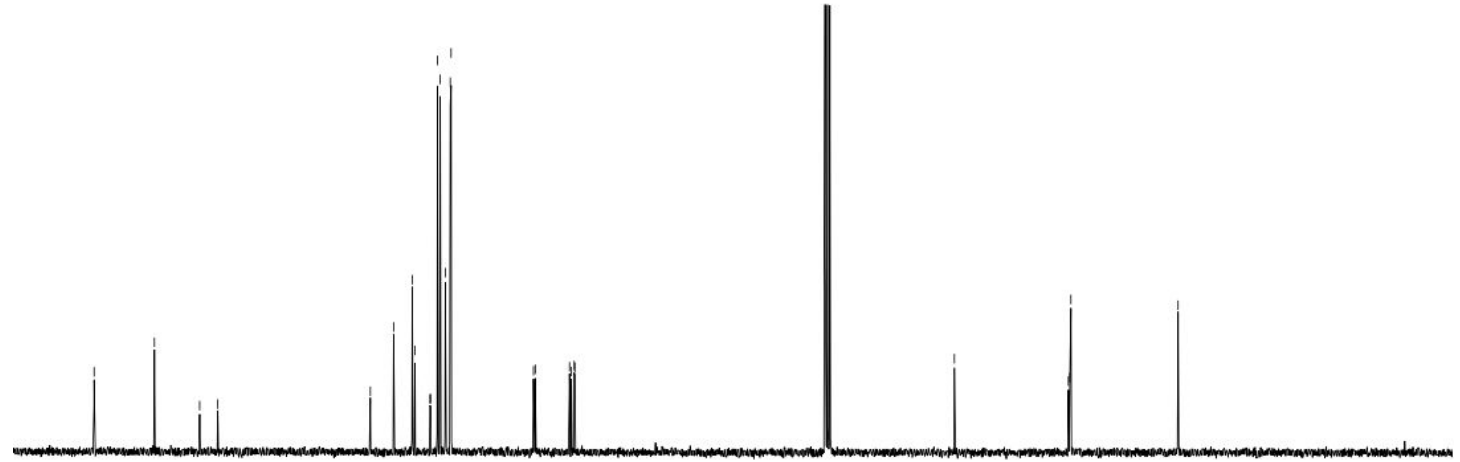

$\begin{array}{lllllllllllllllllll}180 & 170 & 160 & 150 & 140 & 130 & 120 & 110 & 100 & 90 & 80 & 70 & 60 & 50 & 40 & 30 & 20 & 10 & 0\end{array}$

${ }^{1} \mathrm{H},{ }^{19} \mathrm{~F}$ and ${ }^{13} \mathrm{C}$ NMR spectra of $\mathbf{3 d}$

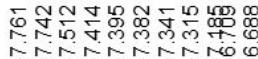

0
0
0

(lll

$3 e$

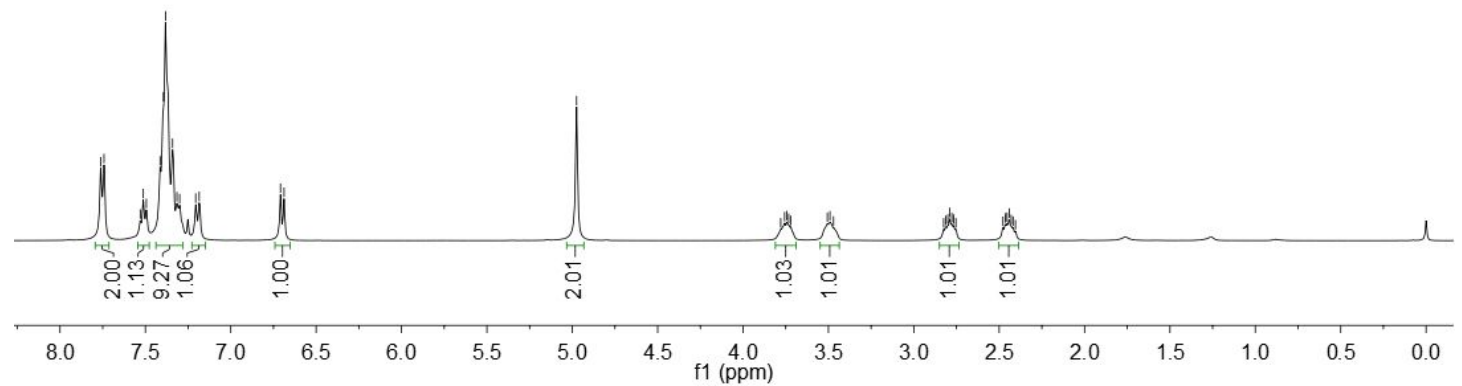



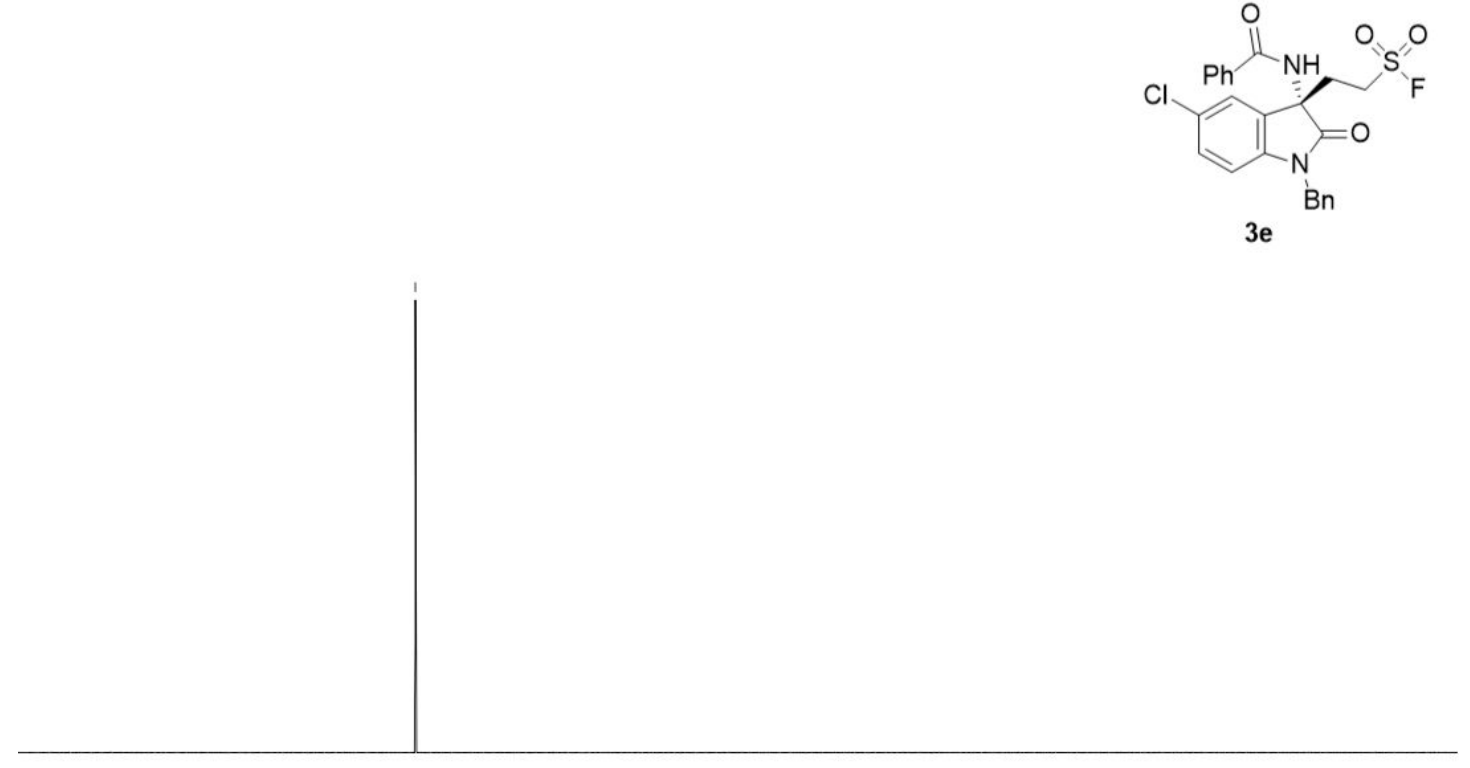

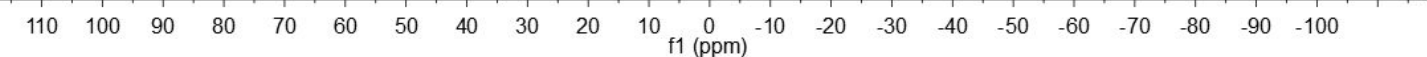

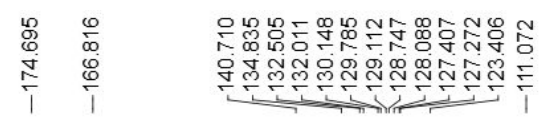

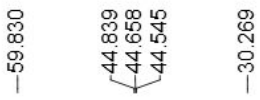

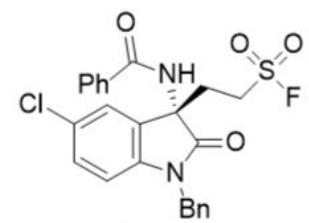

$3 e$

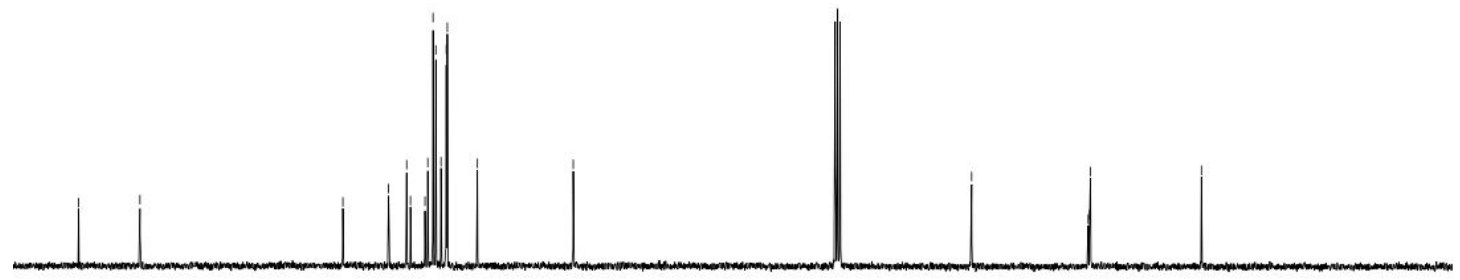

$\begin{array}{llllllllllllllllllll}180 & 170 & 160 & 150 & 140 & 130 & 120 & 110 & 100 & \begin{array}{c}90 \\ \mathrm{f} 1(\mathrm{ppm})\end{array} & 80 & 70 & 60 & 50 & 40 & 30 & 20 & 10 & 0\end{array}$

${ }^{1} \mathrm{H},{ }^{19} \mathrm{~F}$ and ${ }^{13} \mathrm{C}$ NMR spectra of $\mathbf{3 e}$ 


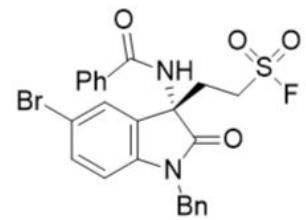

$3 f$

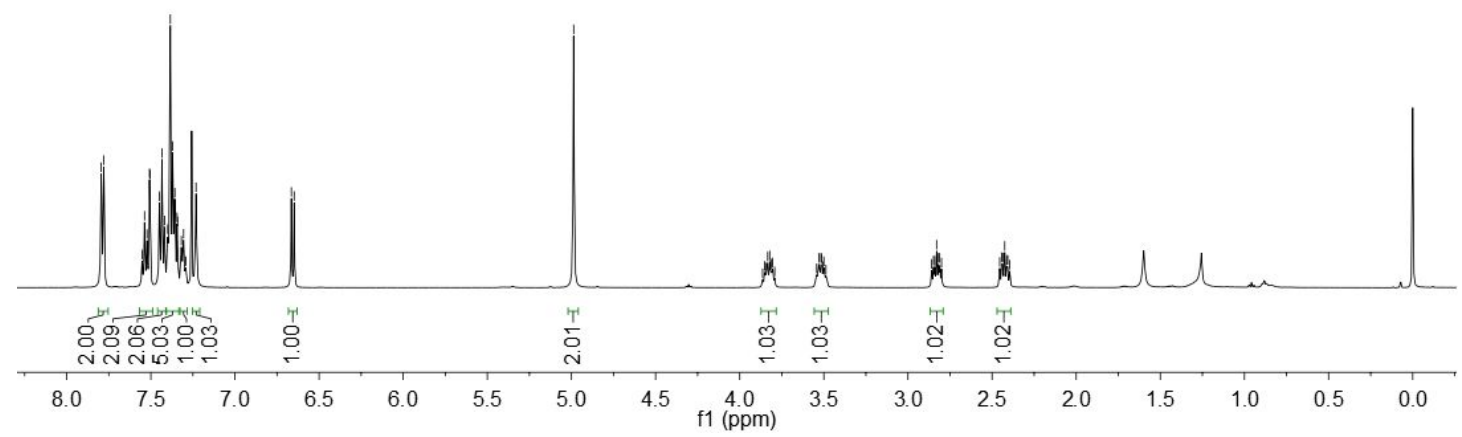

స్心

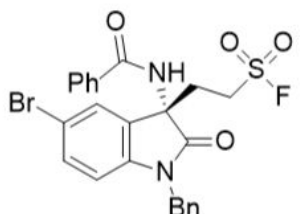

$3 f$

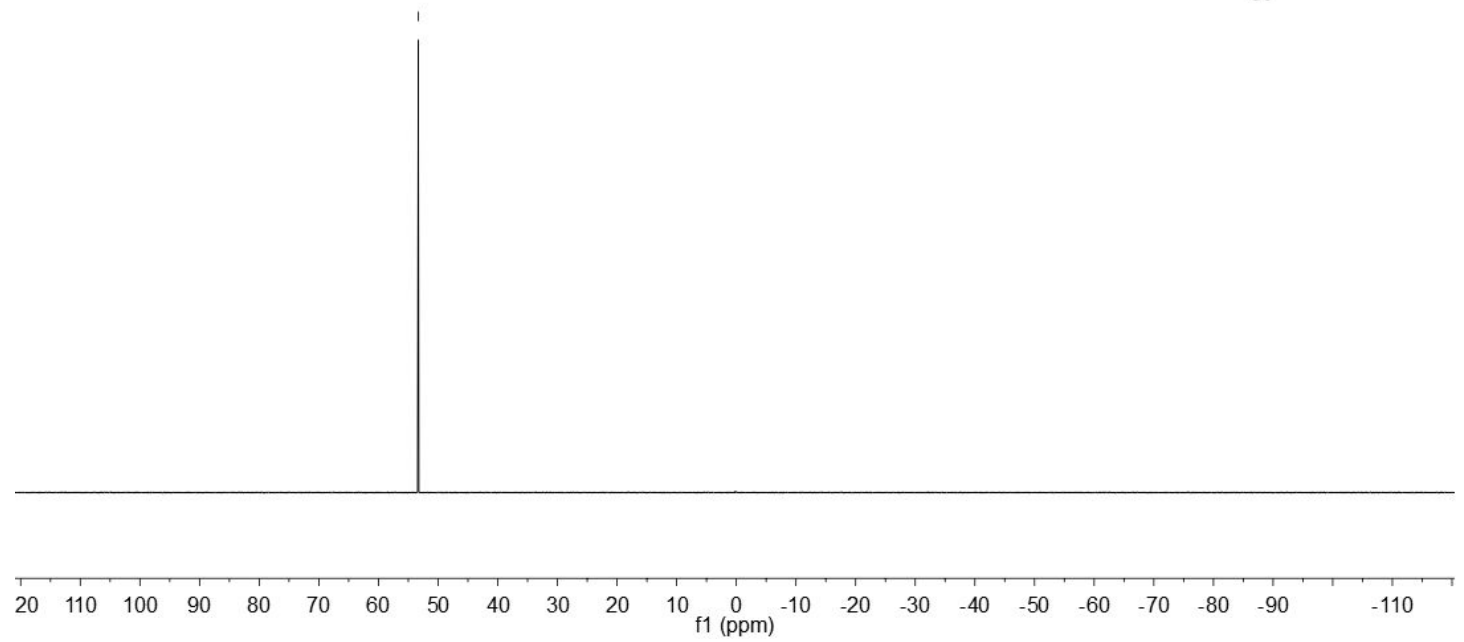




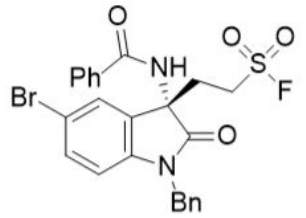

$3 f$
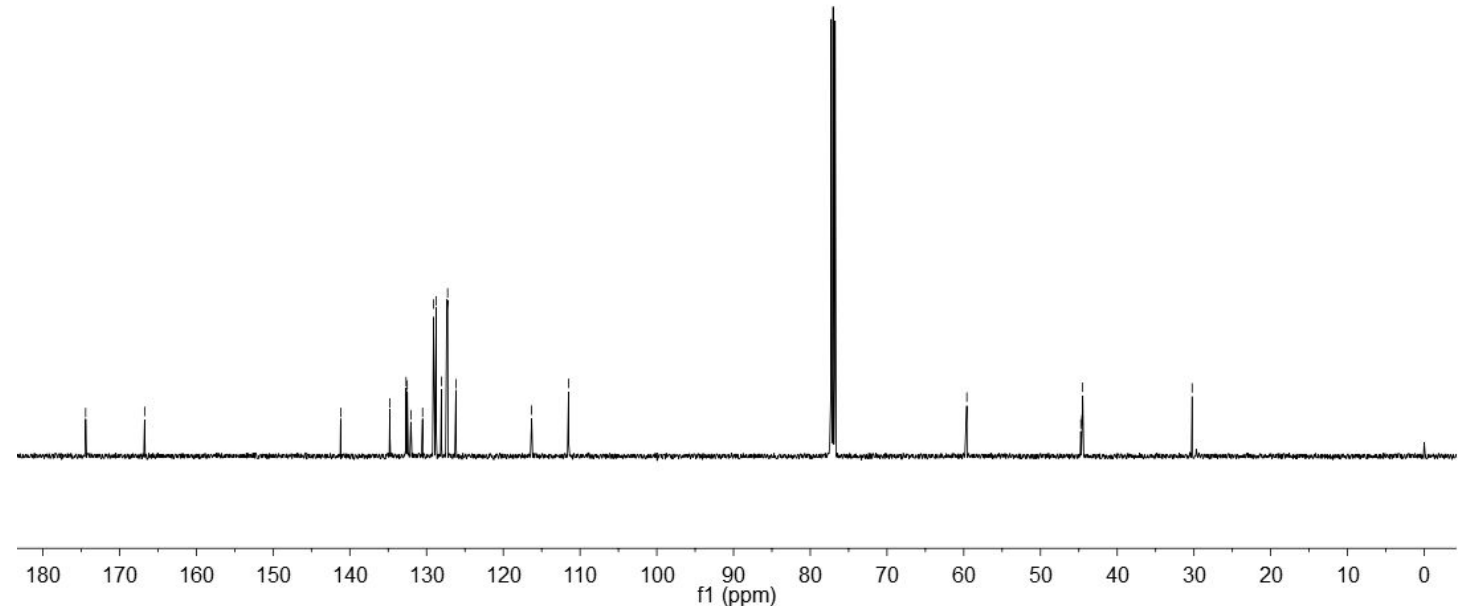

${ }^{1} \mathrm{H},{ }^{19} \mathrm{~F}$ and ${ }^{13} \mathrm{C}$ NMR spectra of $\mathbf{3 f}$

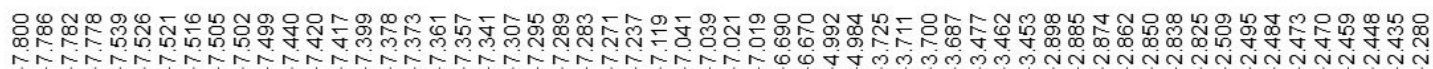

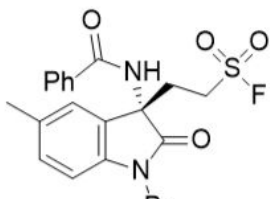

$3 \mathrm{~g}$

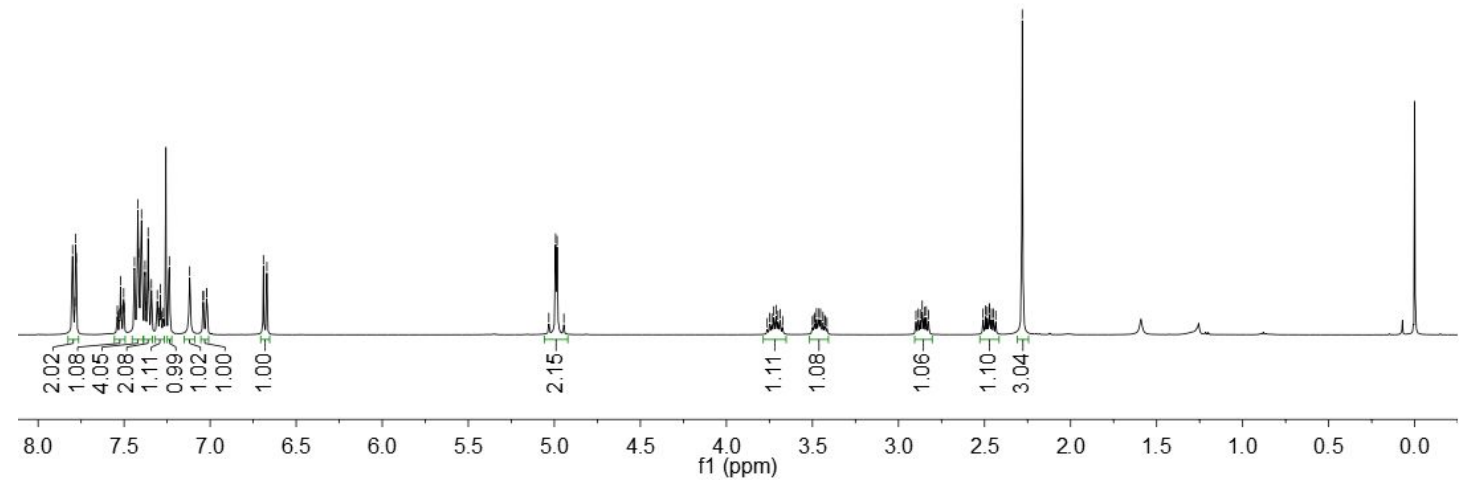




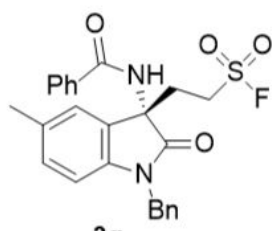

$3 g$

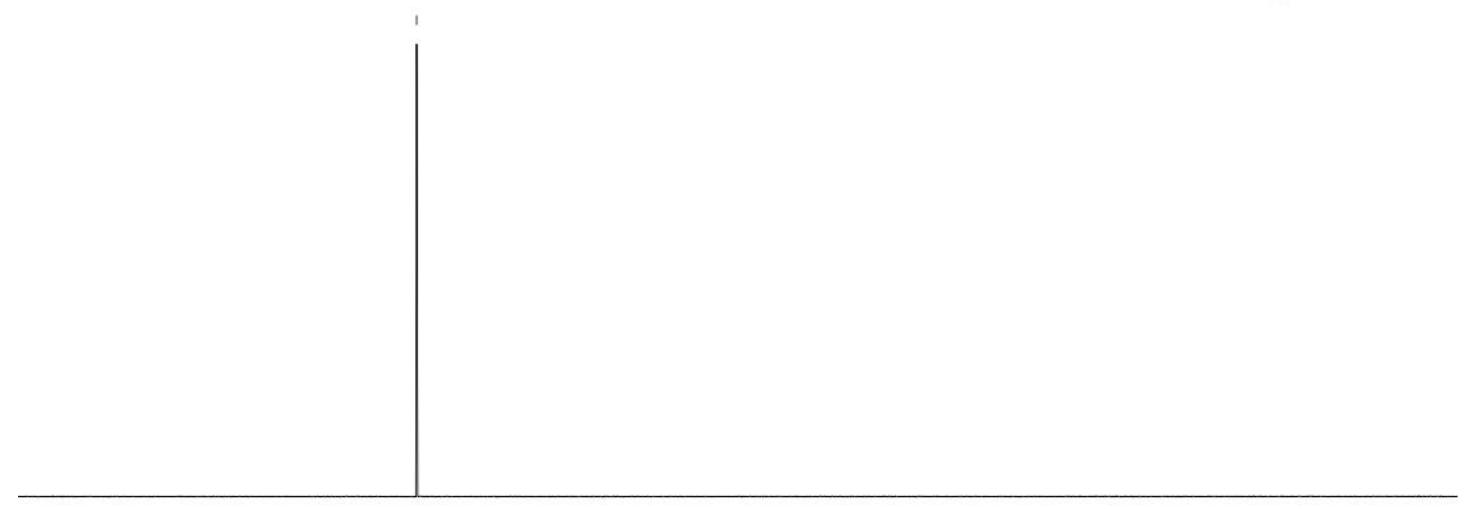

$\begin{array}{lllllllllllllllllllllll}110 & 100 & 90 & 80 & 70 & 60 & 50 & 40 & 30 & 20 & 10 & 0 & 0 & -10 & -20 & -30 & -40 & -50 & -60 & -70 & -80 & -90 & -100\end{array}$

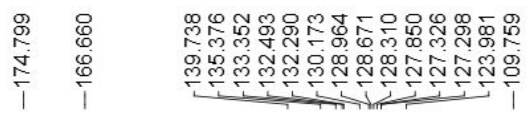

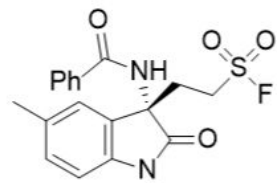

$3 \mathrm{~g}$

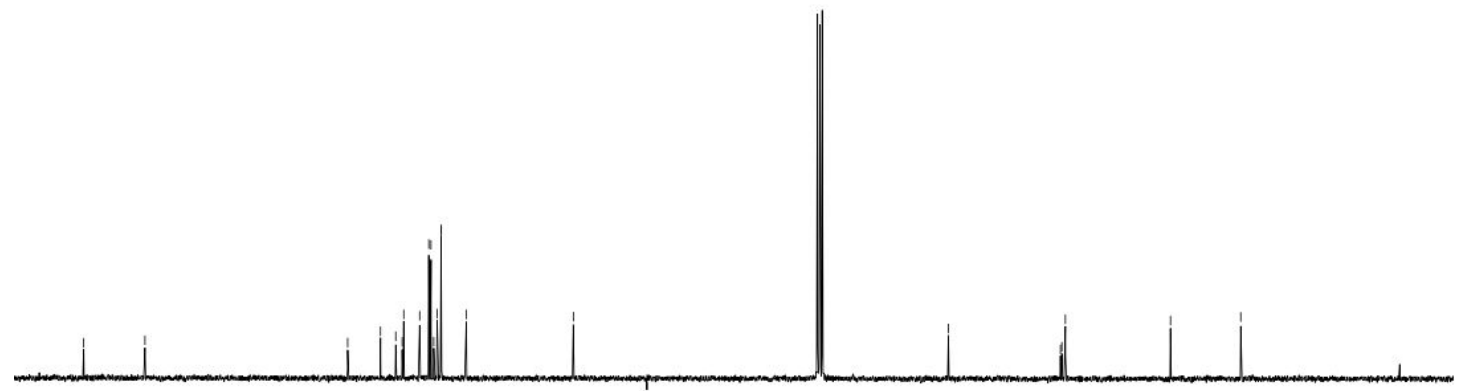

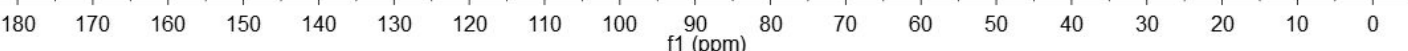

${ }^{1} \mathrm{H},{ }^{19} \mathrm{~F}$ and ${ }^{13} \mathrm{C}$ NMR spectra of $\mathbf{3 g}$ 


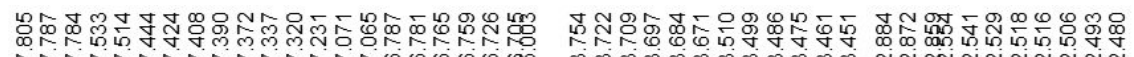

\section{(1)}

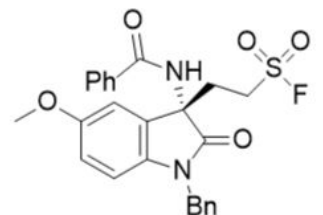

$3 \mathrm{~h}$

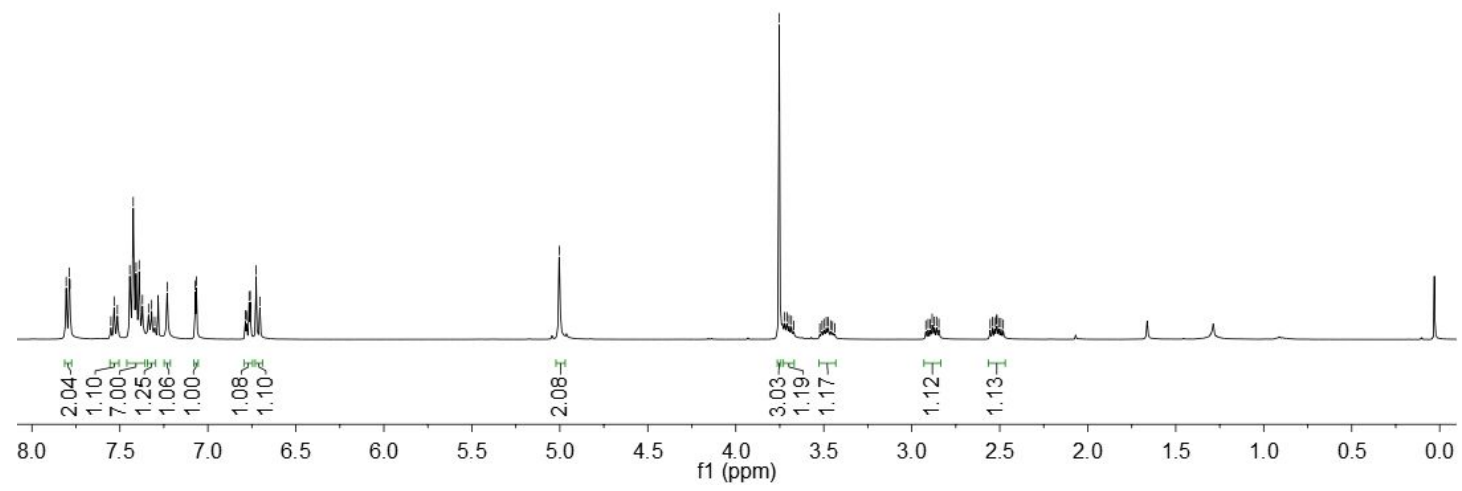

욜

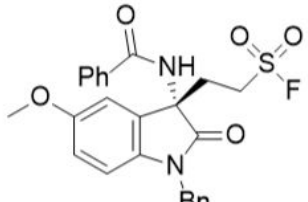

$3 \mathrm{~h}$

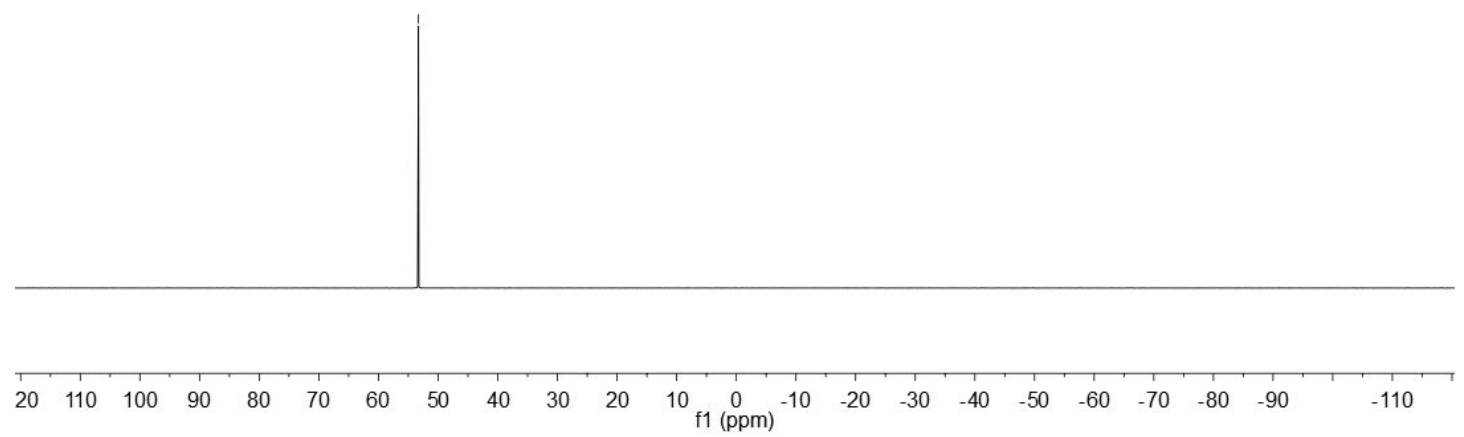




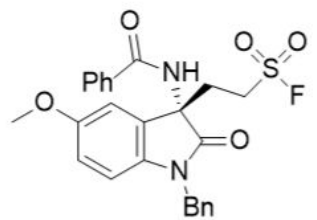

$3 \mathrm{~h}$

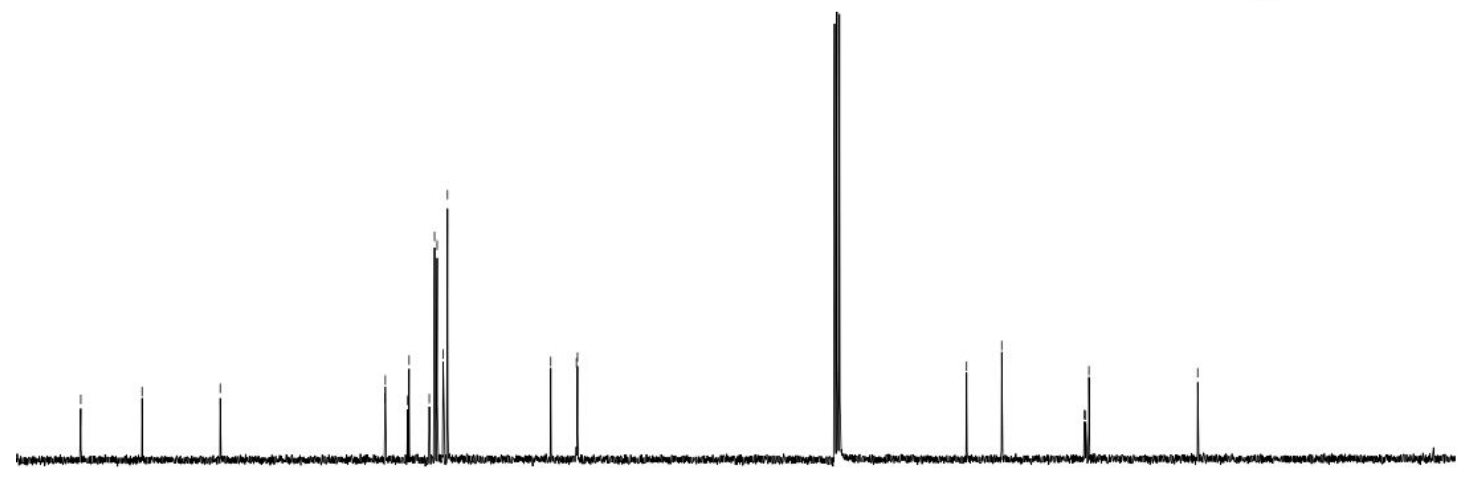

$\begin{array}{llllllllllllllllllll}180 & 170 & 160 & 150 & 140 & 130 & 120 & 110 & 100 & 90 & 80 & 70 & 60 & 50 & 40 & 30 & 20 & 10 & 0\end{array}$

${ }^{1} \mathrm{H},{ }^{19} \mathrm{~F}$ and ${ }^{13} \mathrm{C}$ NMR spectra of $\mathbf{3 h}$

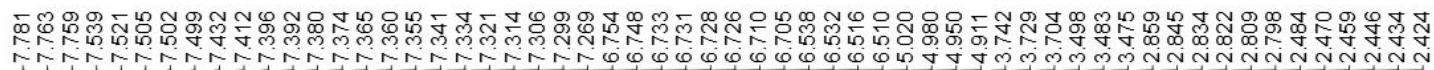
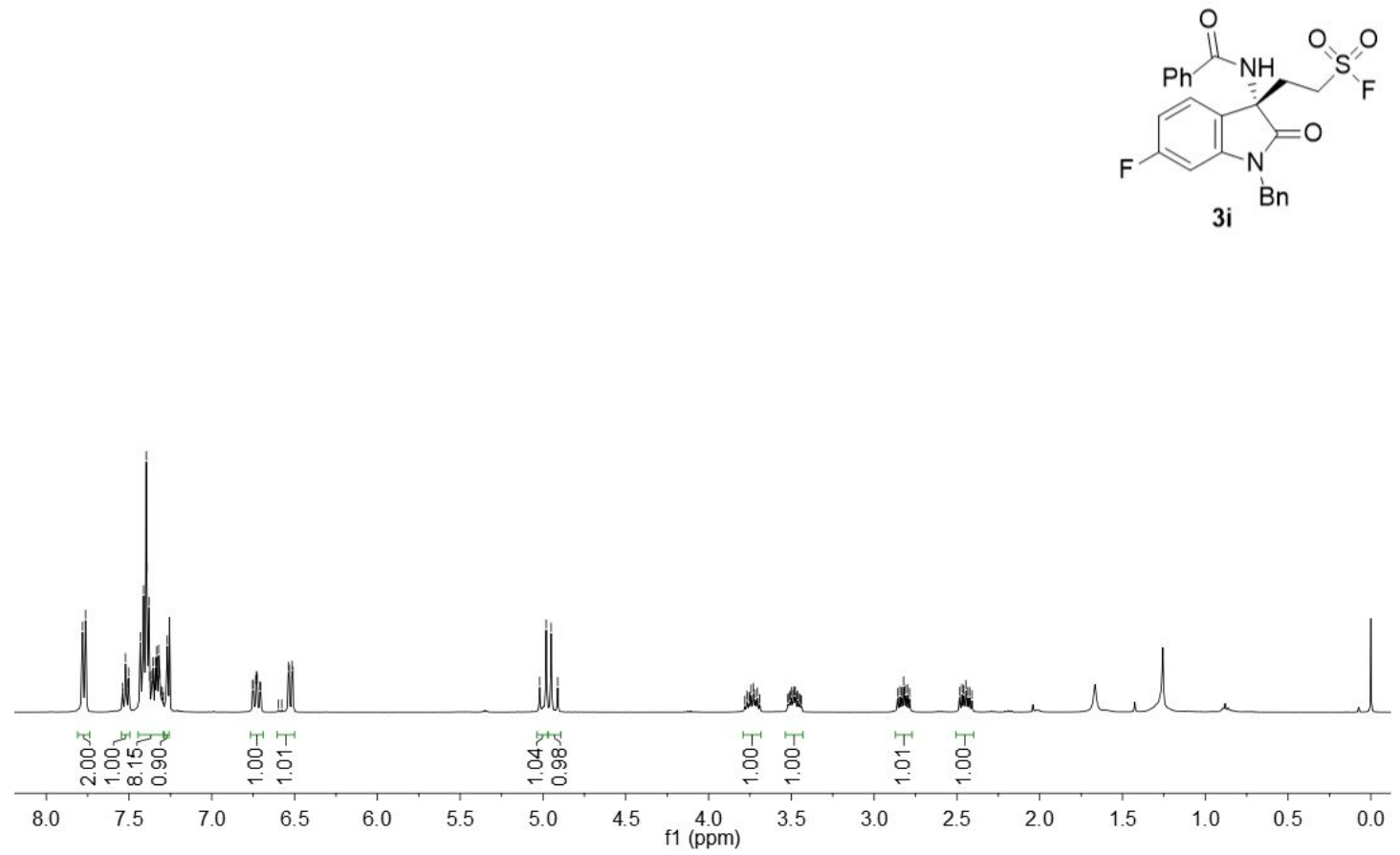


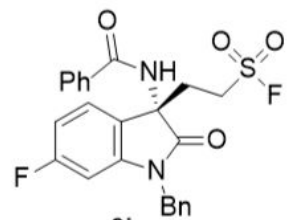

$3 i^{\mathrm{Bn}}$

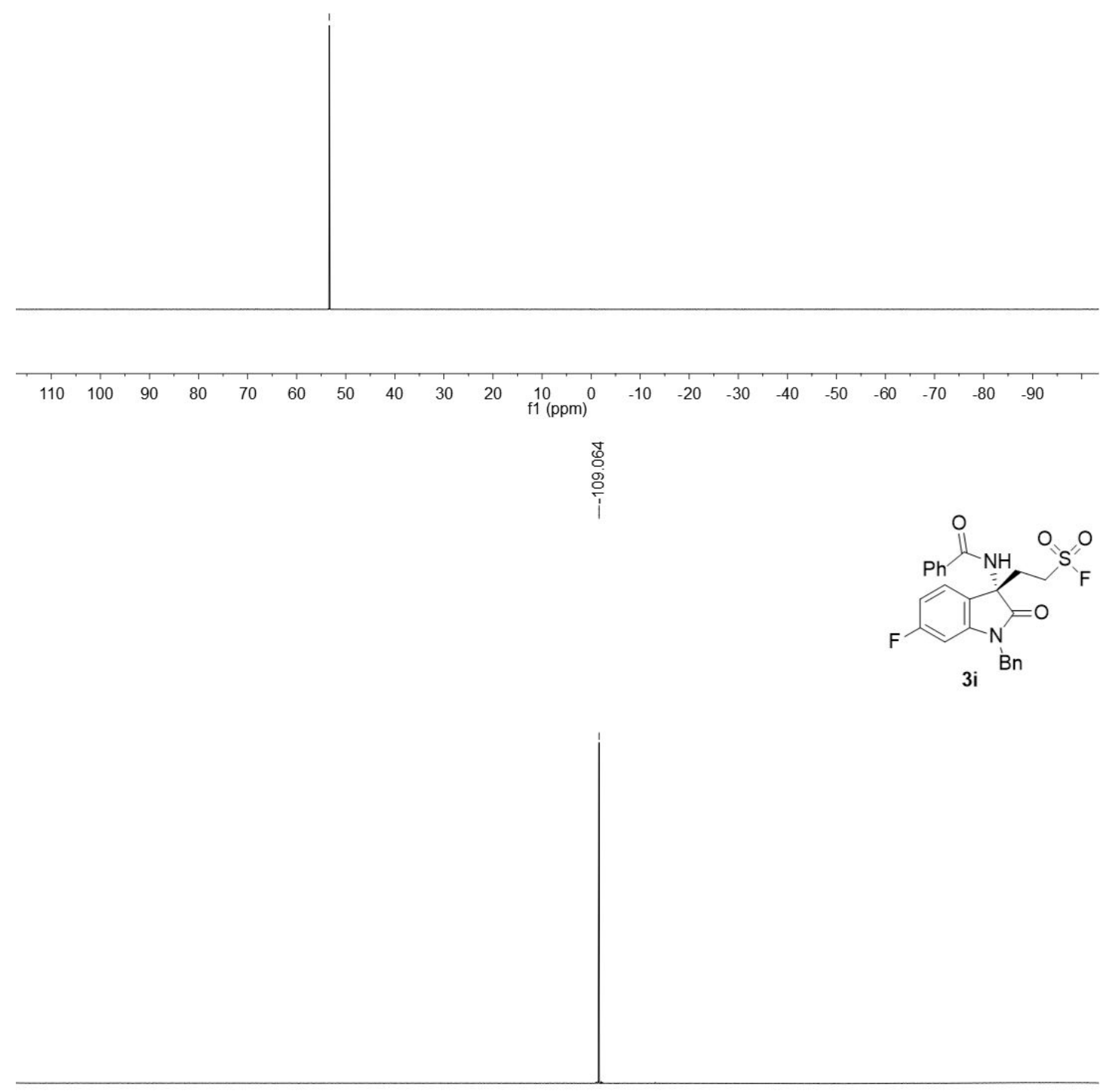

$\begin{array}{llllllllllll}10 & 0 & -10 & -20 & -30 & -40 & -50 & -60 & -70 & -80 & -90 & -100 \\ & & & & & & & & & & & \end{array}$ 

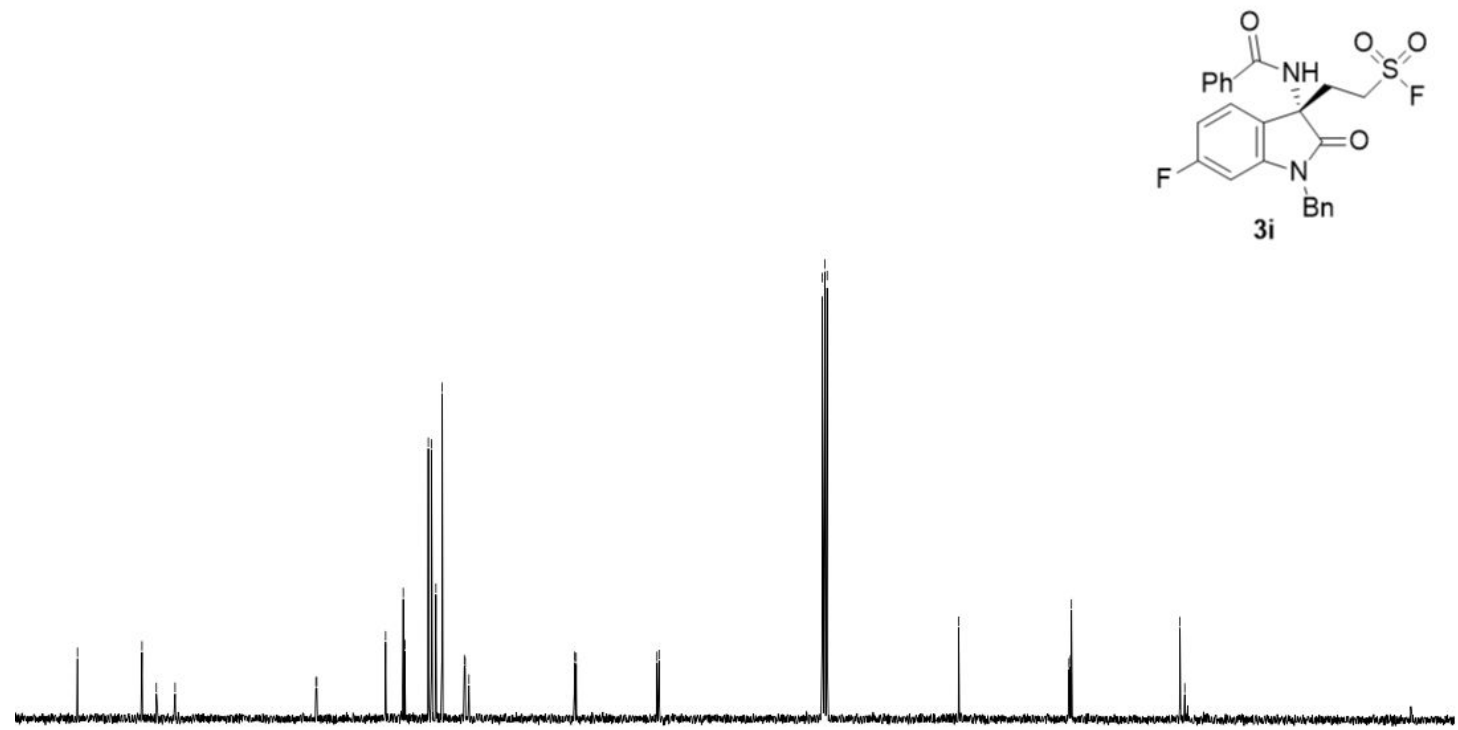

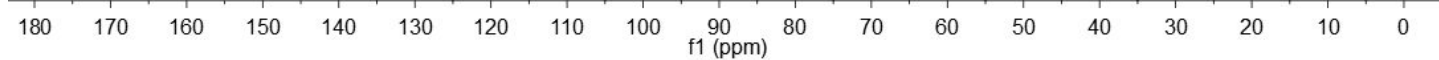

${ }^{1} \mathrm{H},{ }^{19} \mathrm{~F}$ and ${ }^{13} \mathrm{C}$ NMR spectra of $\mathbf{3 i}$

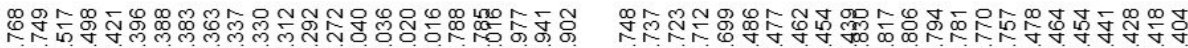

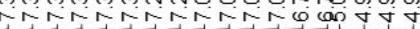

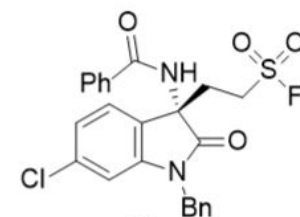

$3 \mathbf{j}$

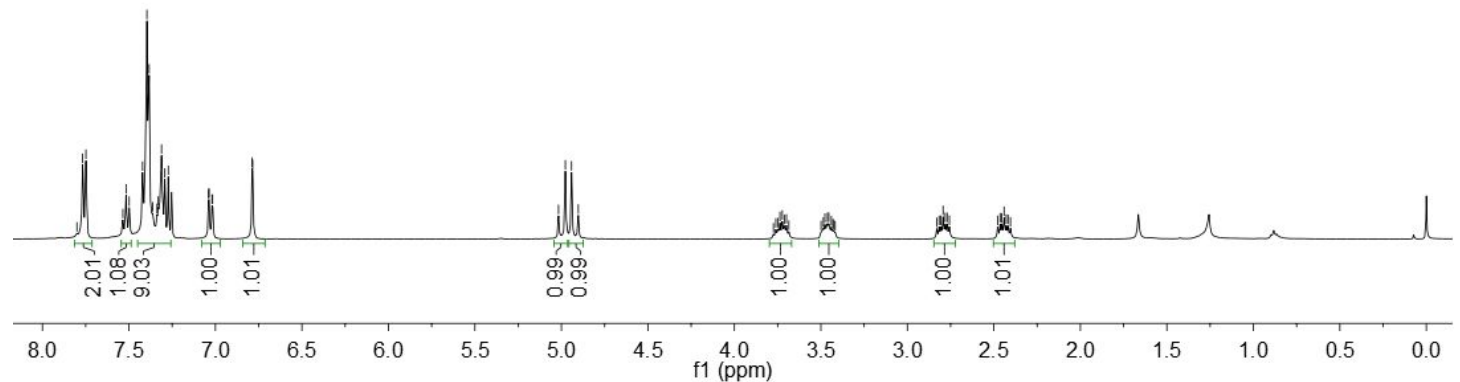



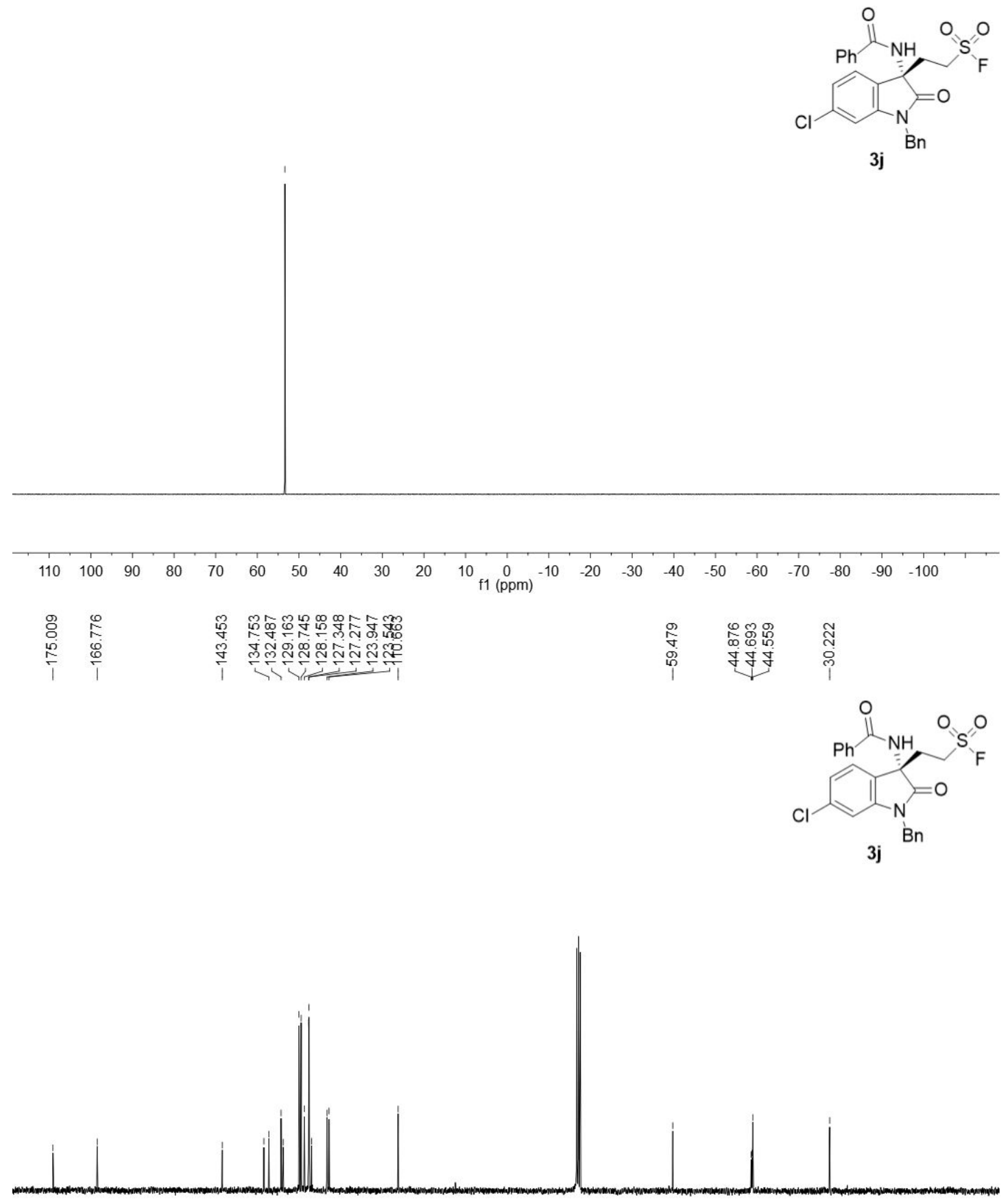

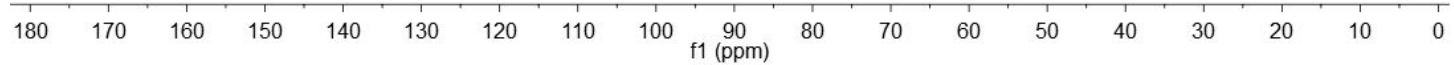

${ }^{1} \mathrm{H},{ }^{19} \mathrm{~F}$ and ${ }^{13} \mathrm{C}$ NMR spectra of $\mathbf{3 j}$ 


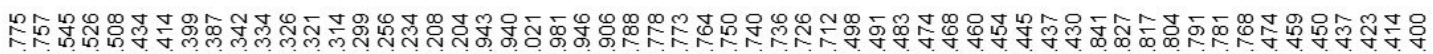
Niñ<smiles>O=C(N[C@@]1(CCS(=O)(=O)O)C(=O)N(Br)c2cc(Br)ccc21)c1ccccc1</smiles>

$3 k$

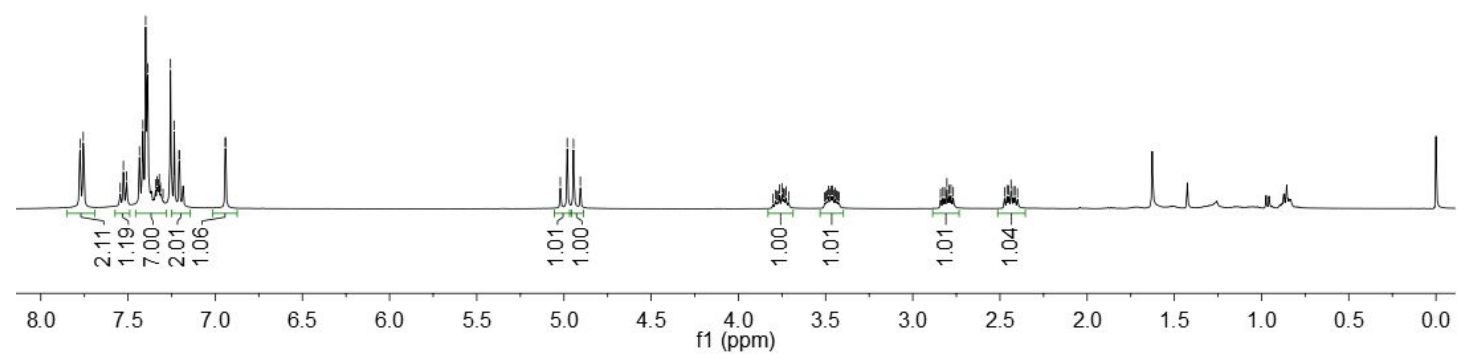

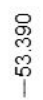
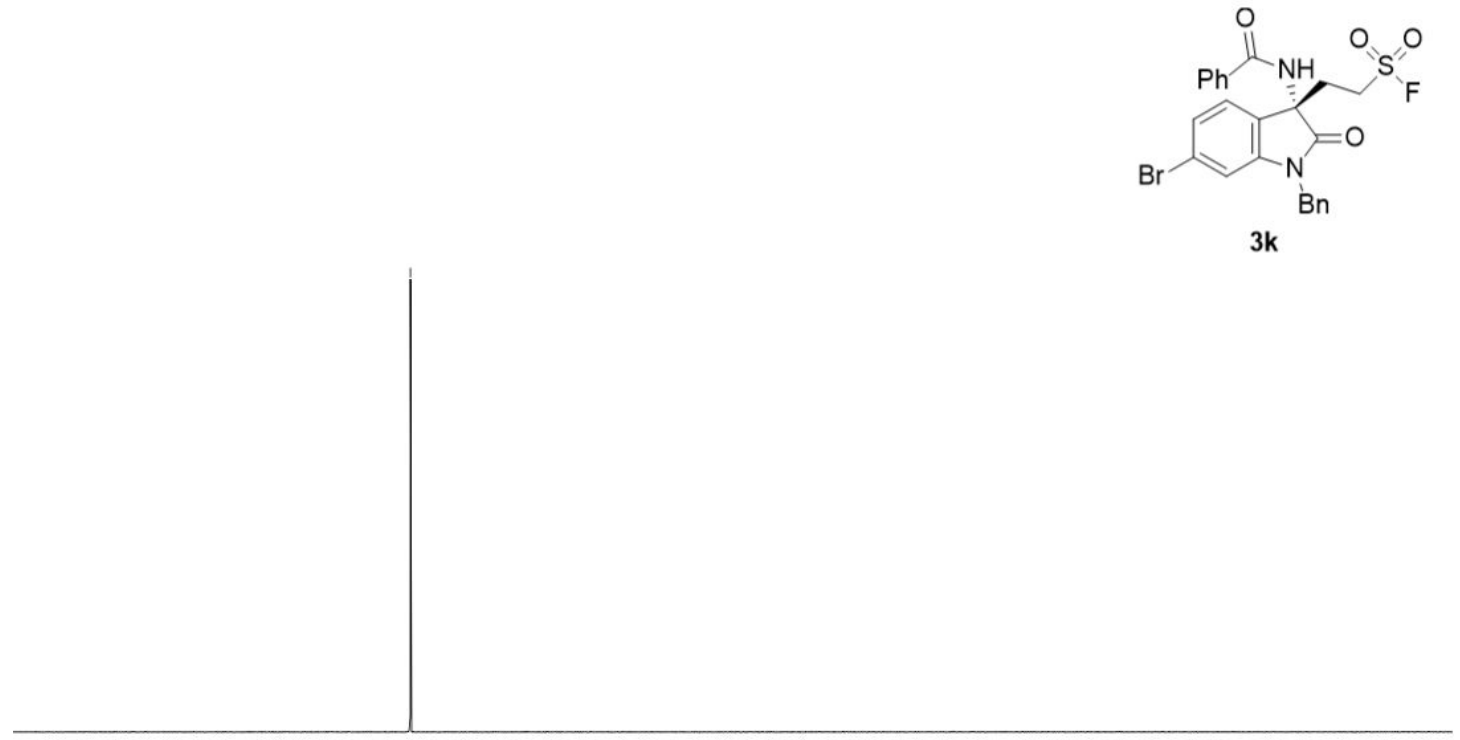

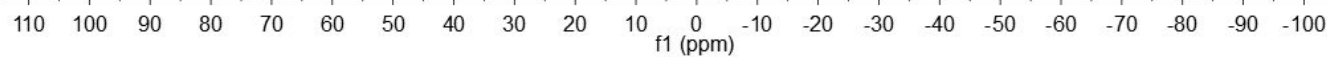




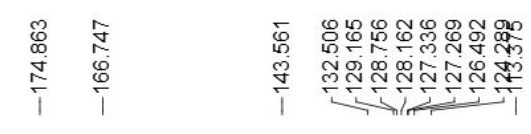

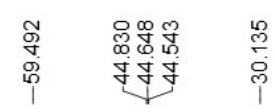

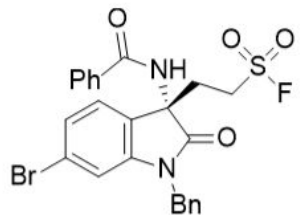

$3 \mathbf{k}$

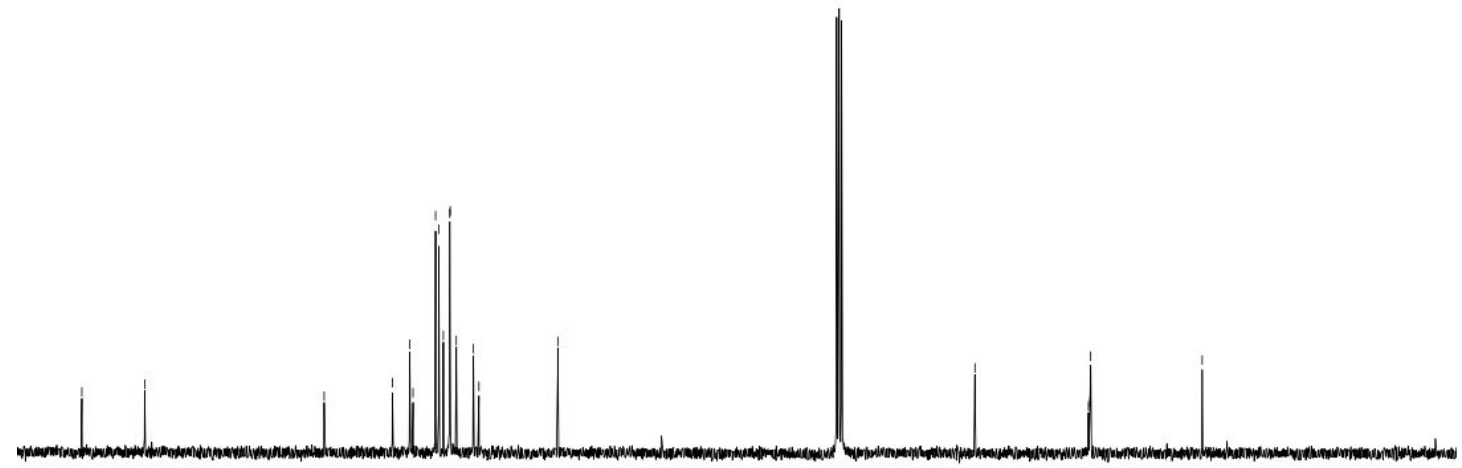

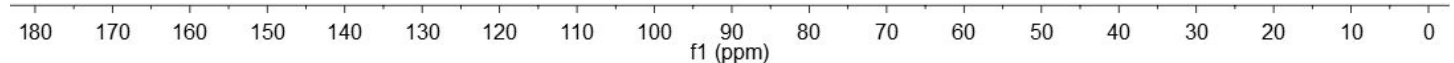

${ }^{1} \mathrm{H},{ }^{19} \mathrm{~F}$ and ${ }^{13} \mathrm{C}$ NMR spectra of $\mathbf{3 k}$

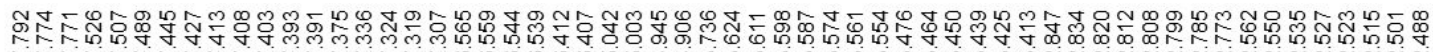
aning

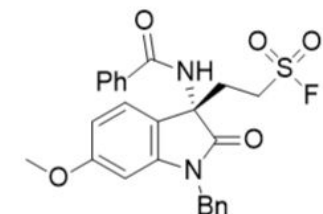

31

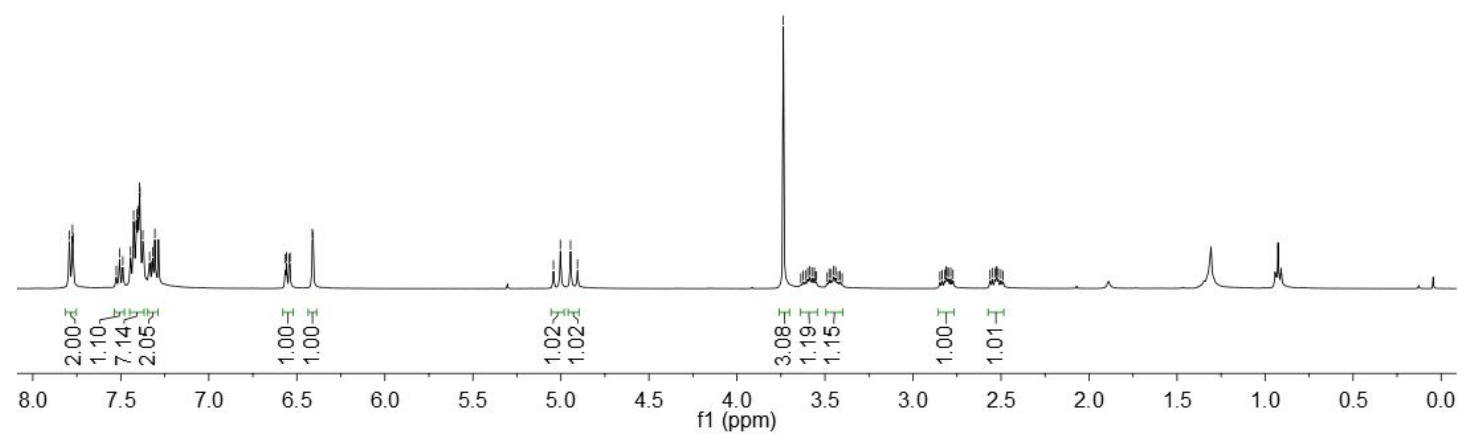




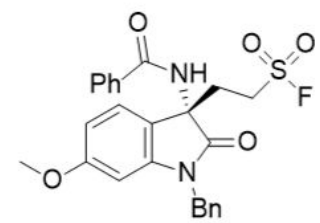

3!

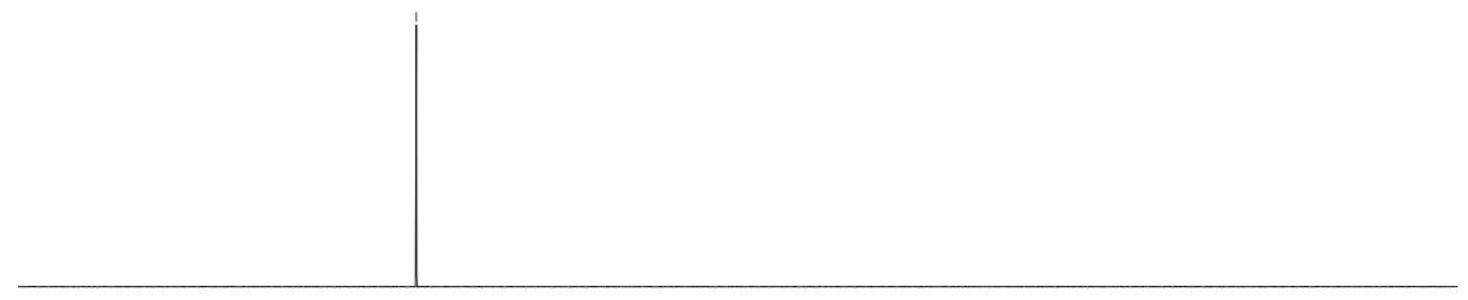

$\begin{array}{lllllllllllllllllllll}110 & 100 & 90 & 80 & 70 & 60 & 50 & 40 & 30 & 20 & 10 & 0 & 0 \\ \mathrm{f} 1(\mathrm{ppm}) & -10 & -20 & -30 & -40 & -50 & -60 & -70 & -80 & -90 & -100 & \end{array}$

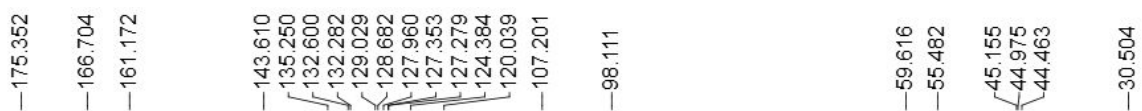

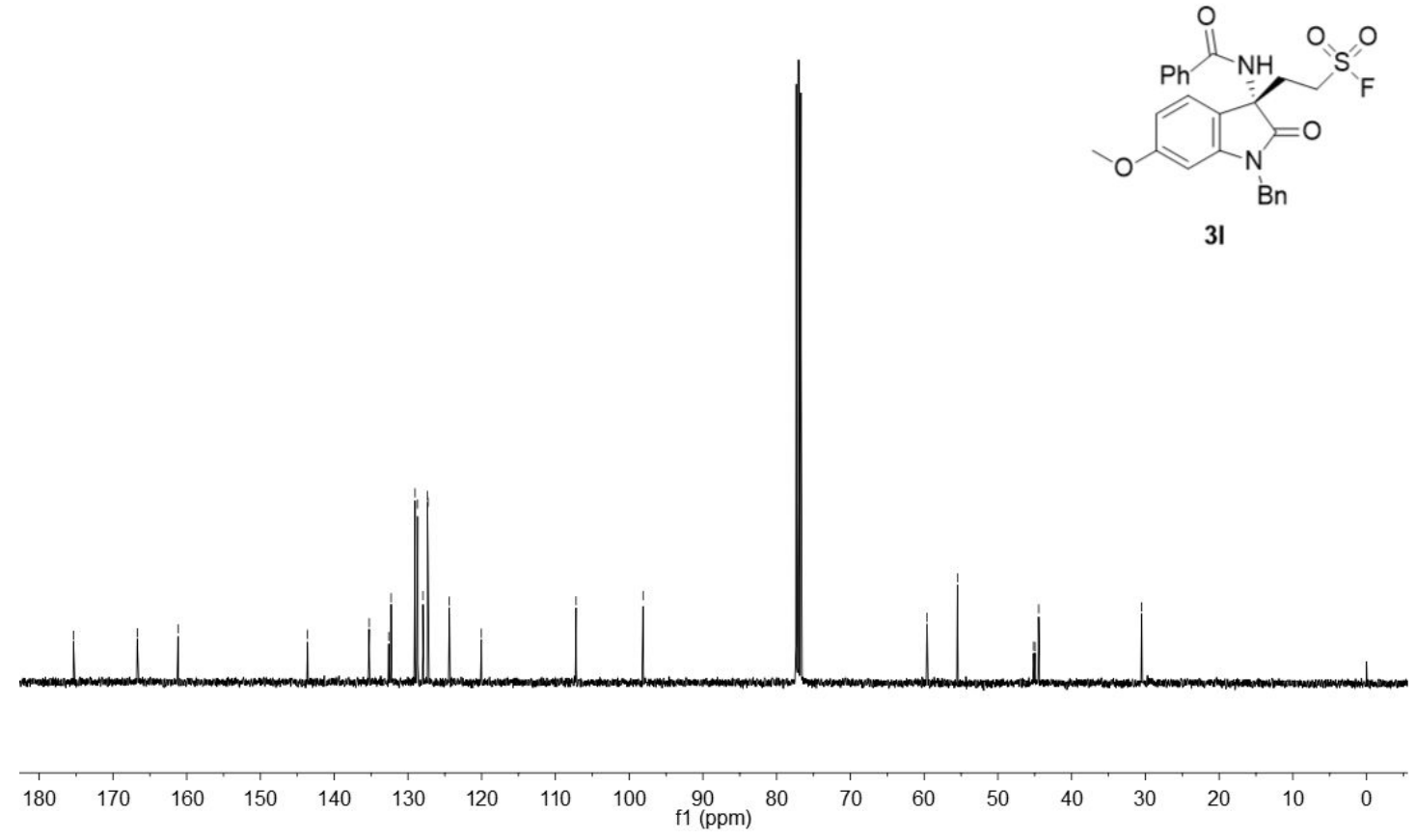

${ }^{1} \mathrm{H},{ }^{19} \mathrm{~F}$ and ${ }^{13} \mathrm{C}$ NMR spectra of $\mathbf{3 l}$ 


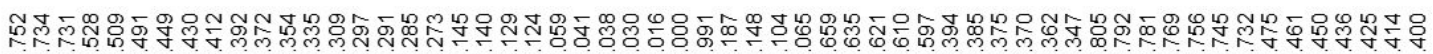

NNTn
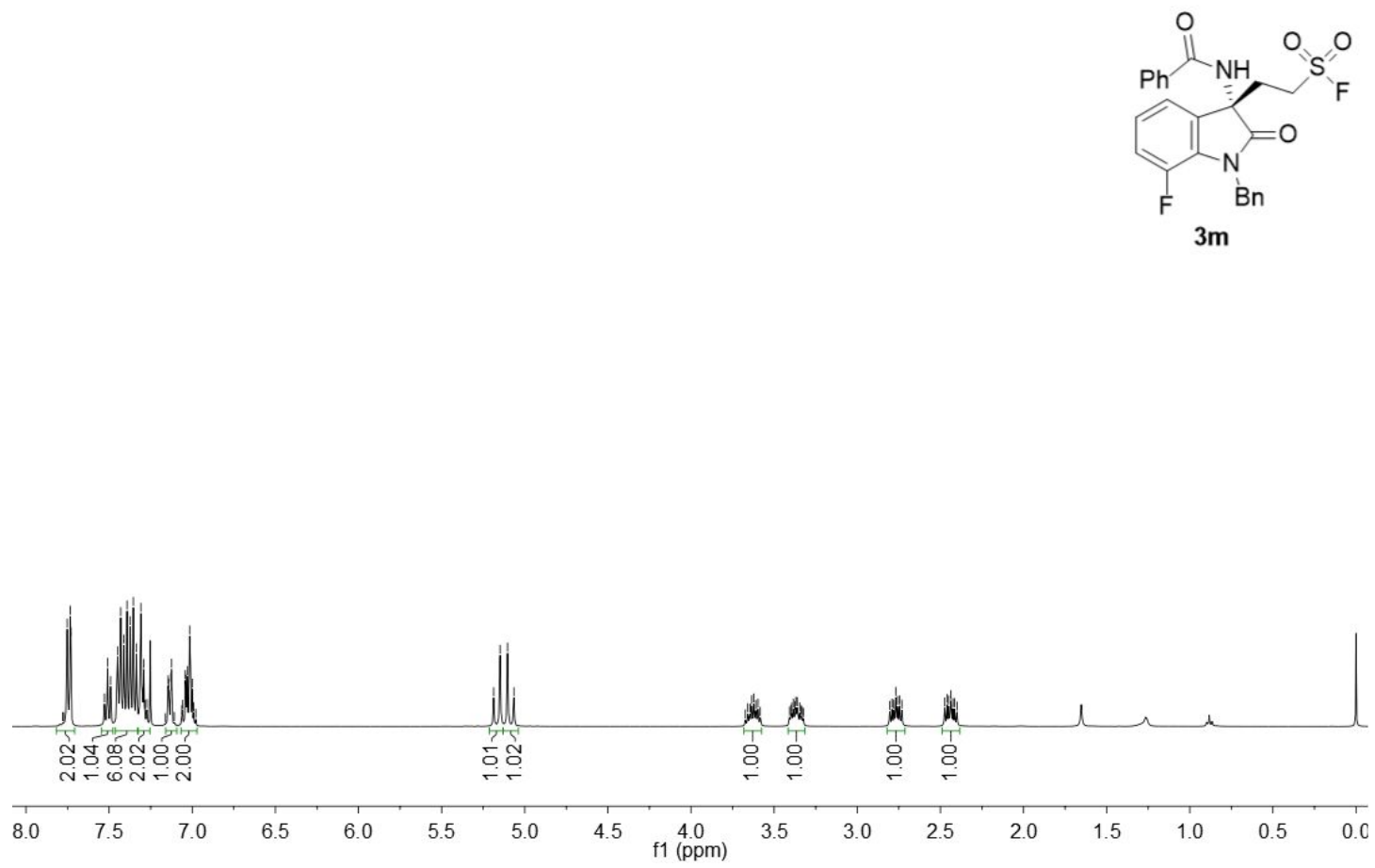

$\stackrel{\substack{m \\ \stackrel{n}{i}}}{i}$

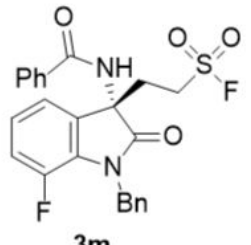

$3 m$

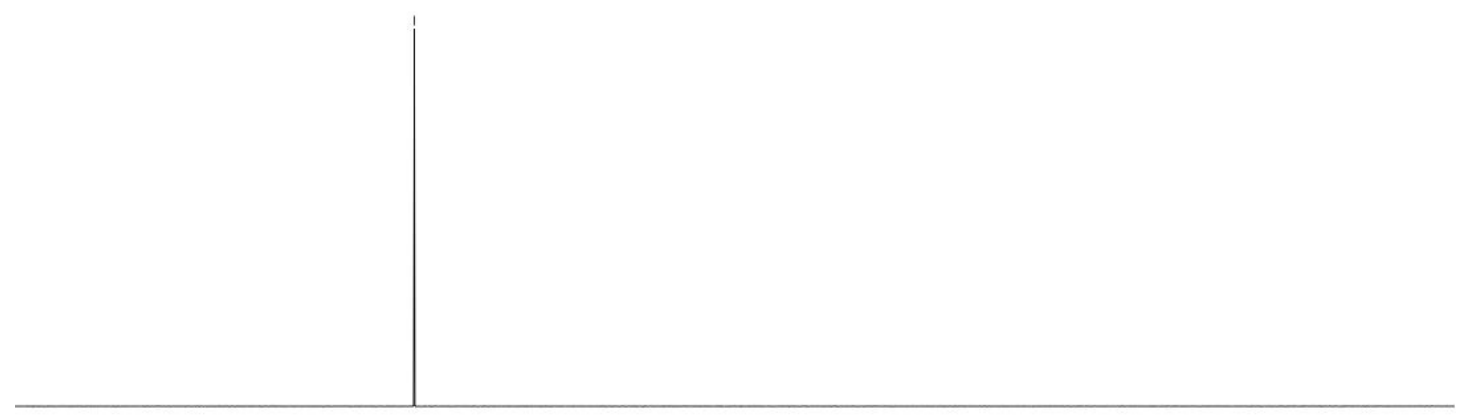

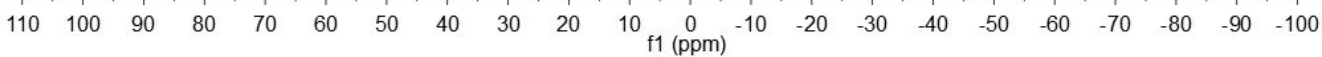



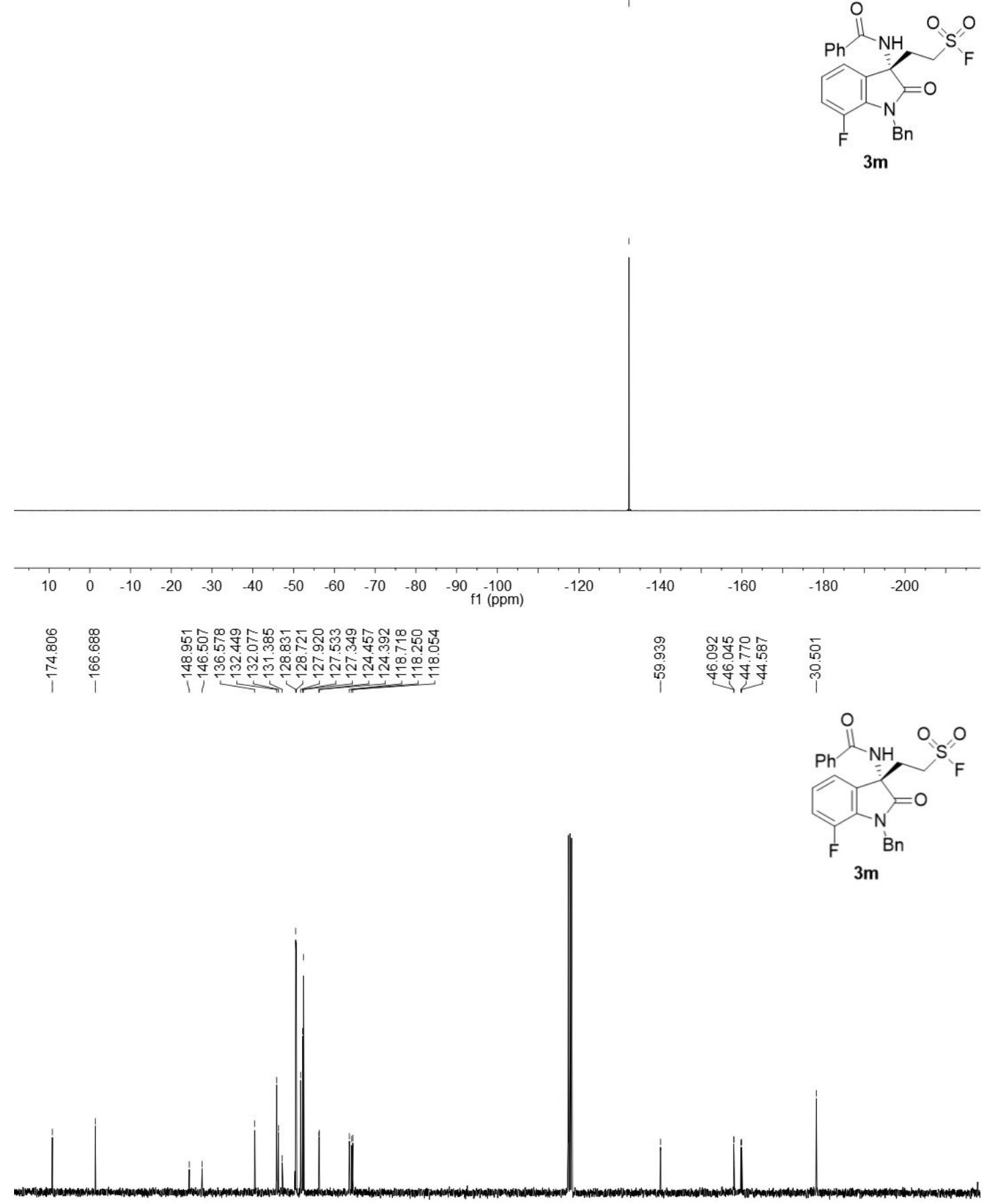

\begin{tabular}{lllllllllllllllllllll}
\hline 180 & 170 & 160 & 150 & 140 & 130 & 120 & 110 & 100 & $\begin{array}{c}90 \\
\mathrm{f} 1(\mathrm{ppm})\end{array}$ & 80 & 70 & 60 & 50 & 40 & 30 & 20 & 10 & 0
\end{tabular}

${ }^{1} \mathrm{H},{ }^{19} \mathrm{~F}$ and ${ }^{13} \mathrm{C}$ NMR spectra of $\mathbf{3 m}$ 


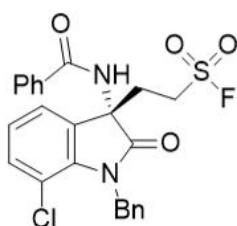

$3 n$

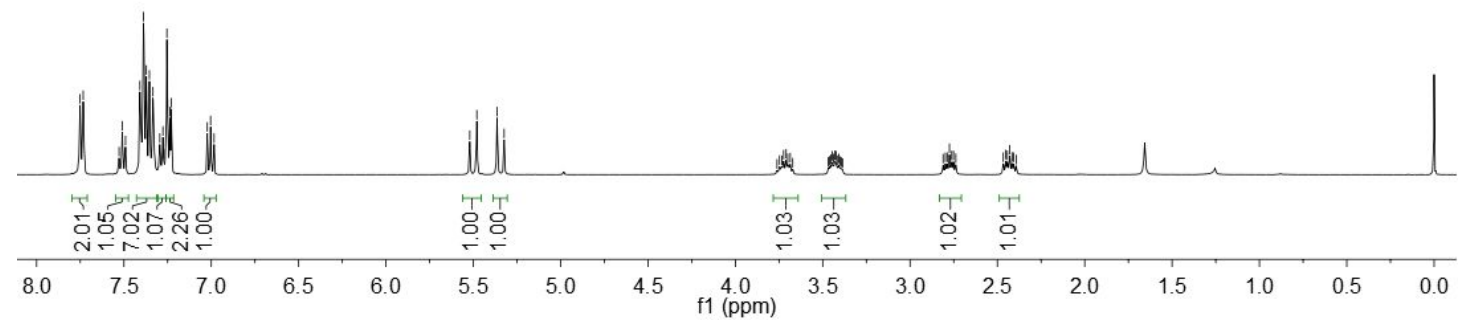

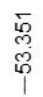

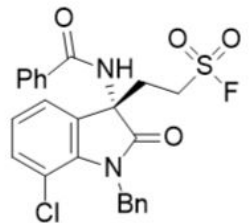

$3 n$

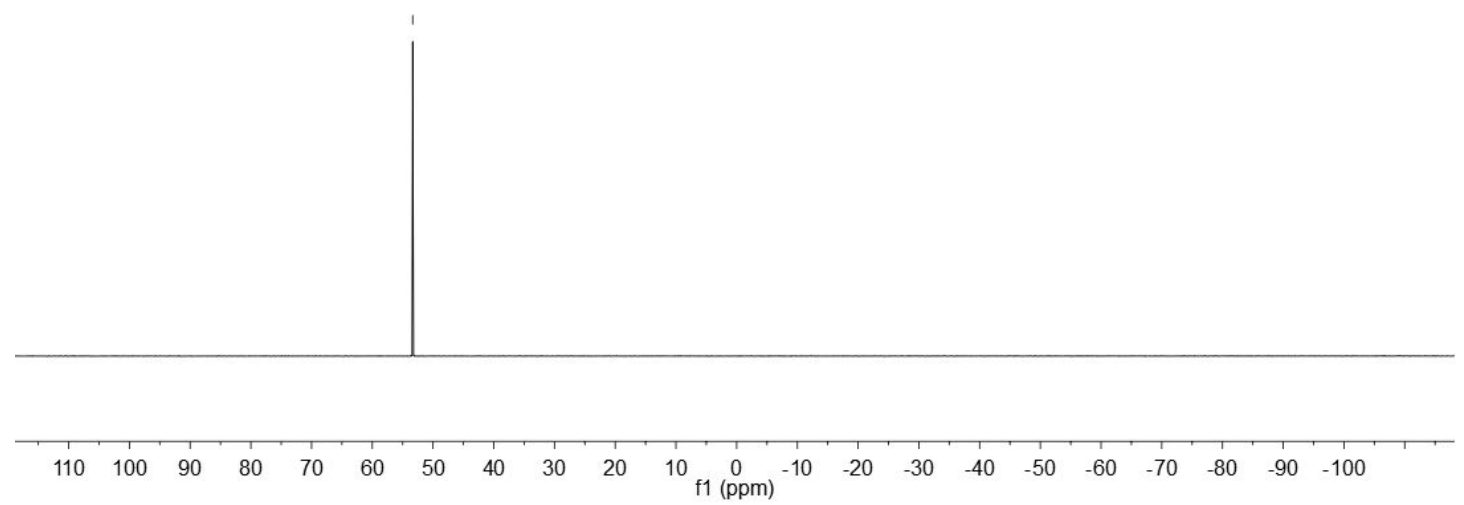




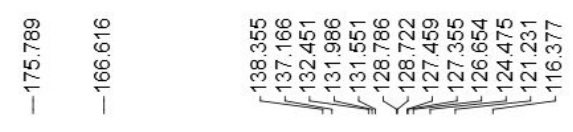

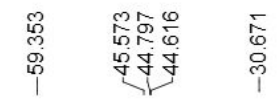

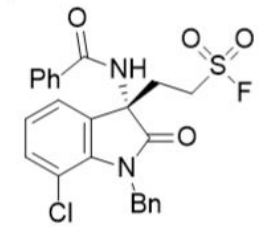

$3 n$
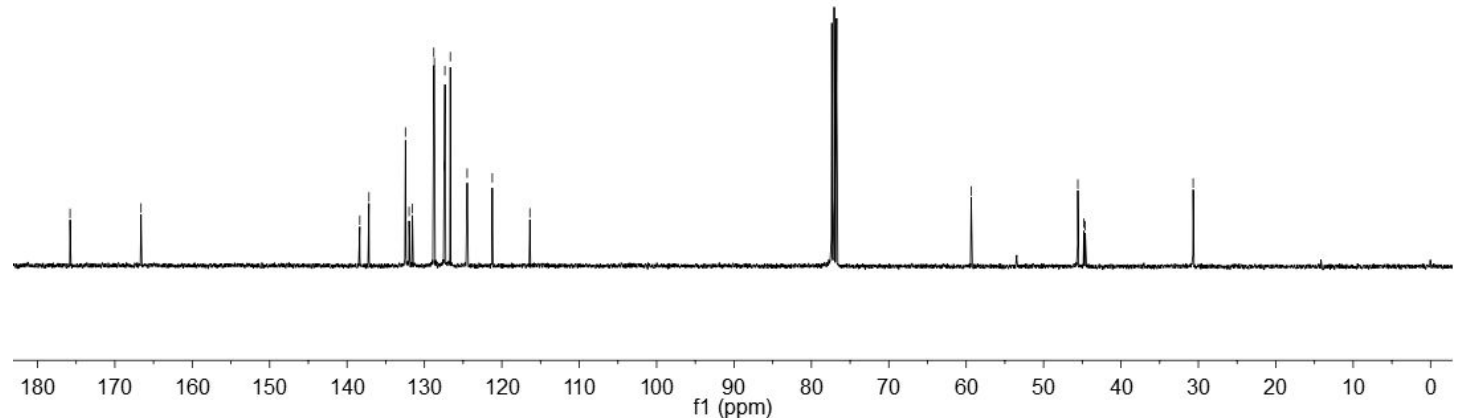

${ }^{1} \mathrm{H},{ }^{19} \mathrm{~F}$ and ${ }^{13} \mathrm{C}$ NMR spectra of $\mathbf{3 n}$

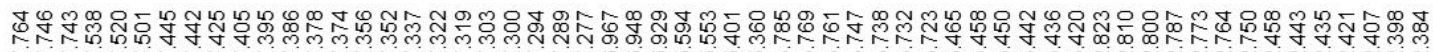

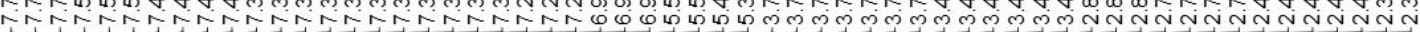<smiles>O=C(NC1(CCS(=O)(=O)F)C(=O)N(Cc2ccccc2)c2c(Br)cccc21)c1ccccc1</smiles>

3o

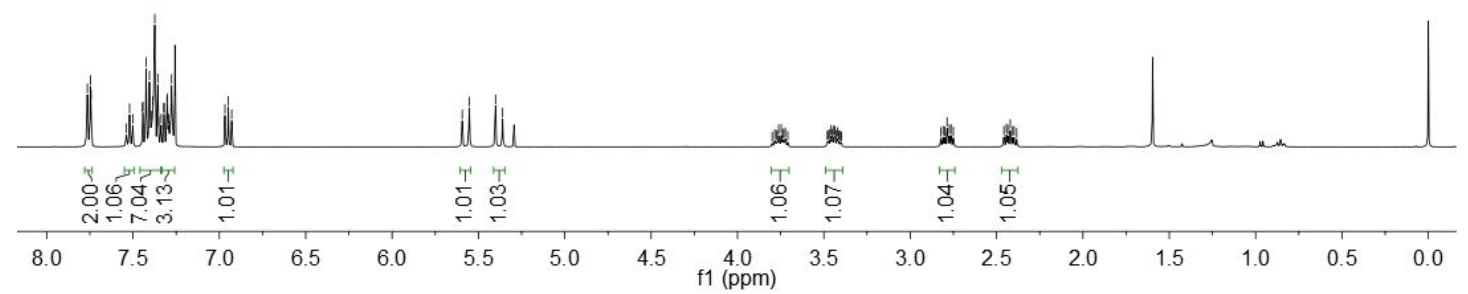




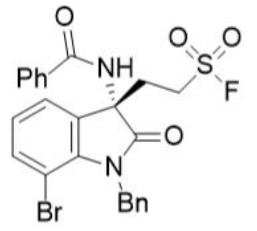

30

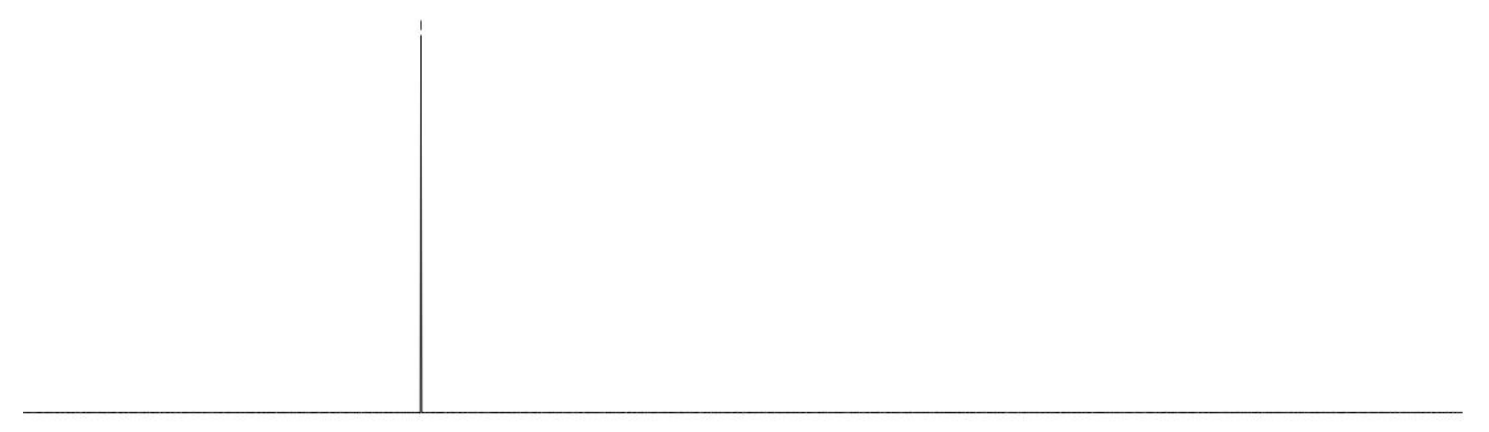

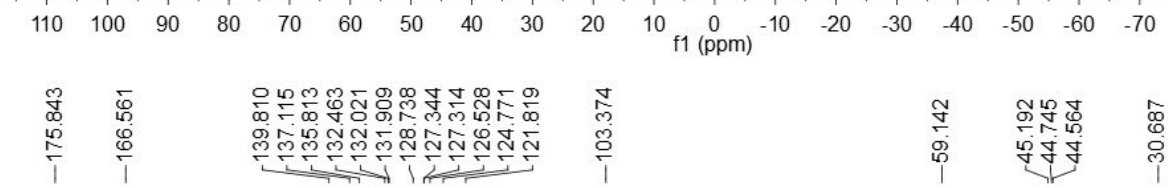

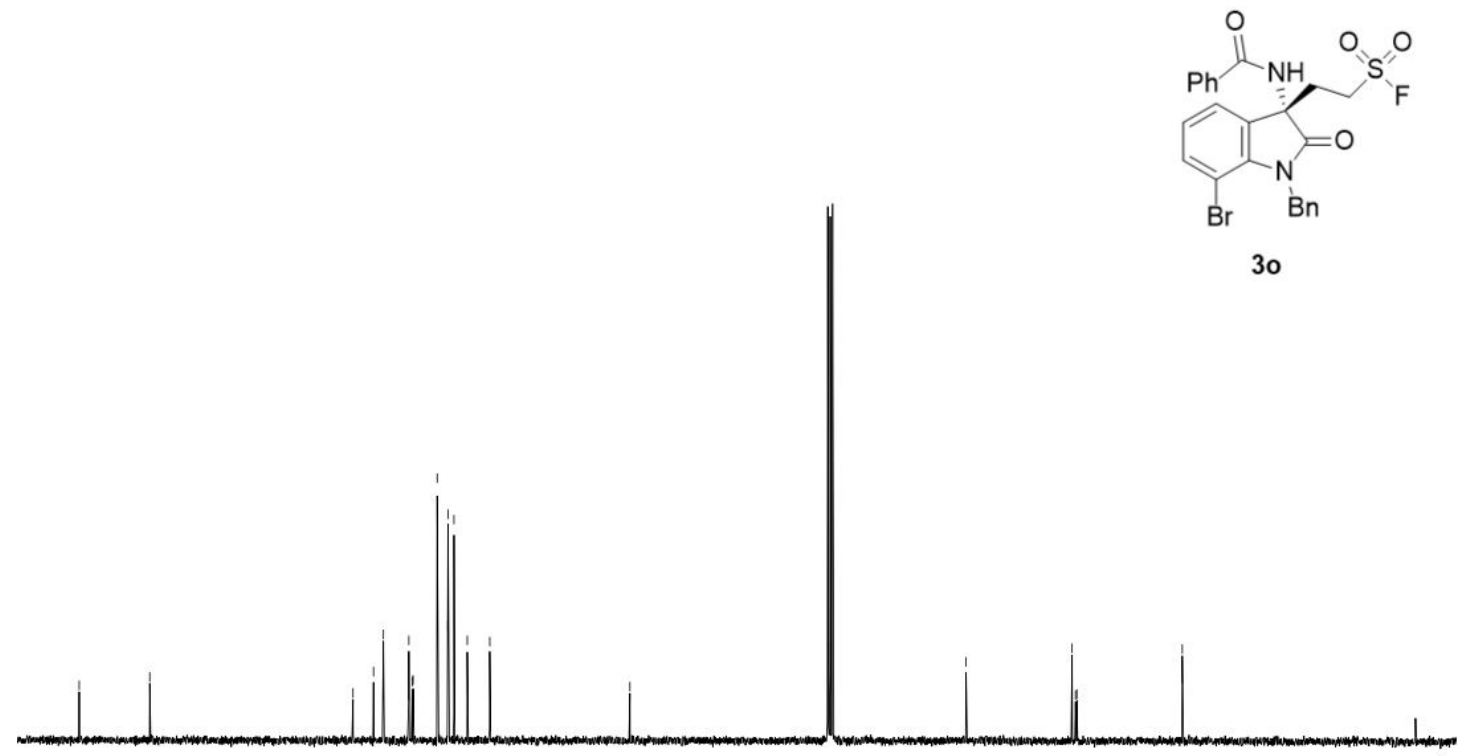

$\begin{array}{lllllllllllllllllllll}180 & 170 & 160 & 150 & 140 & 130 & 120 & 110 & 100 & \underset{\mathrm{f} 1}{9(\mathrm{ppm})} & 80 & 70 & 60 & 50 & 40 & 30 & 20 & 10 & 0\end{array}$

${ }^{1} \mathrm{H},{ }^{19} \mathrm{~F}$ and ${ }^{13} \mathrm{C}$ NMR spectra of $\mathbf{3 o}$ 


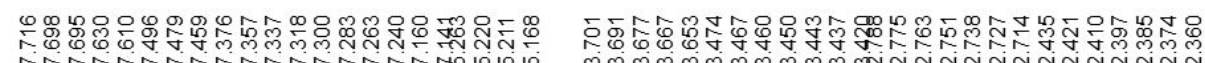

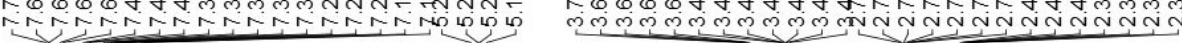

(N)

$3 p$

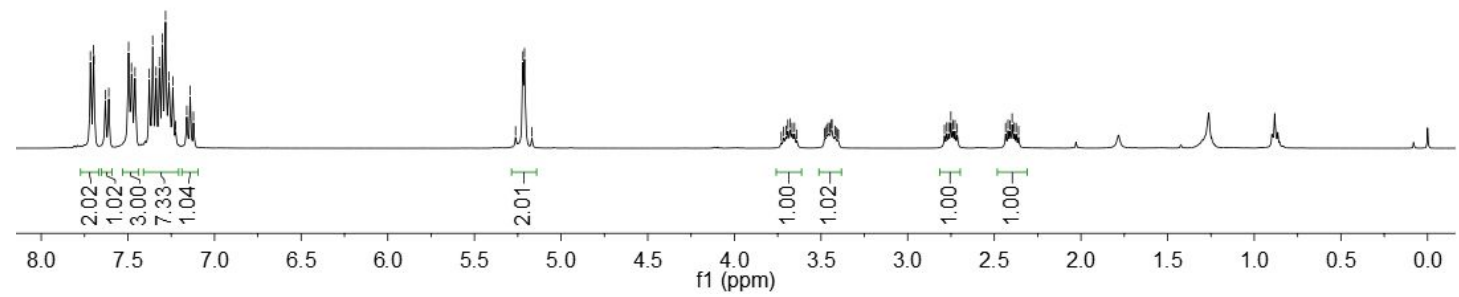

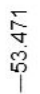

ণิ

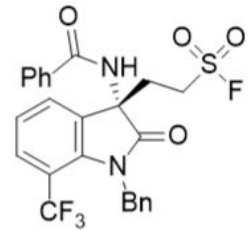

$3 p$

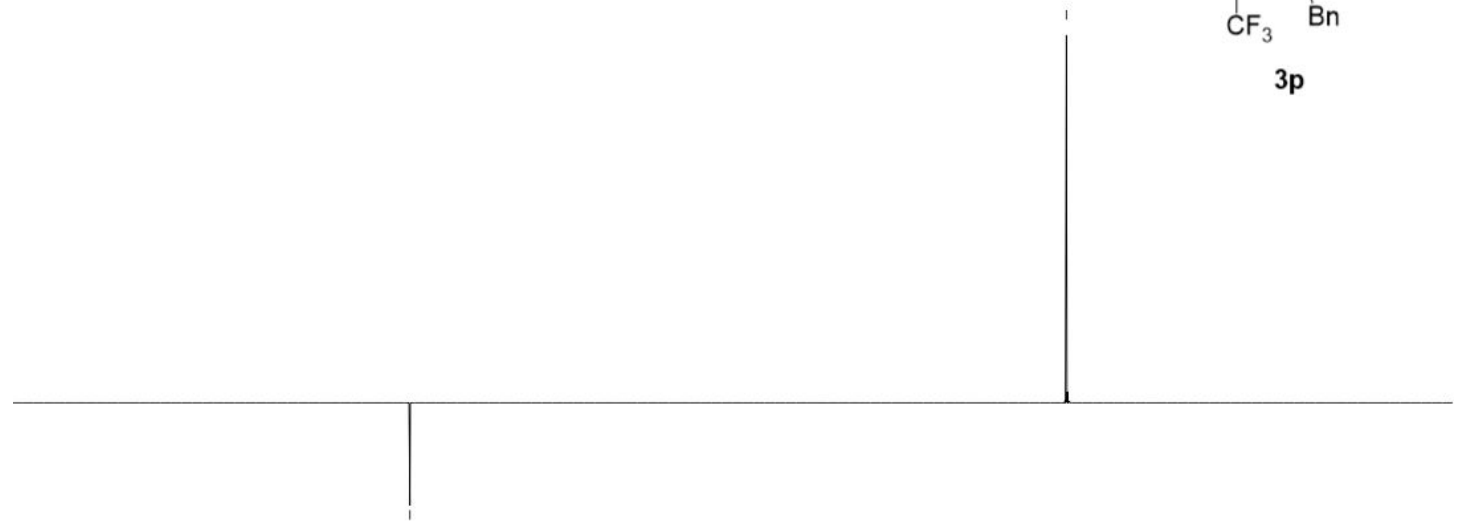

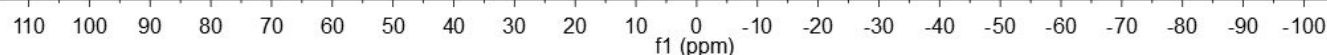




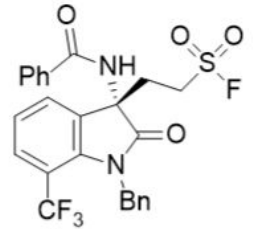

$3 p$
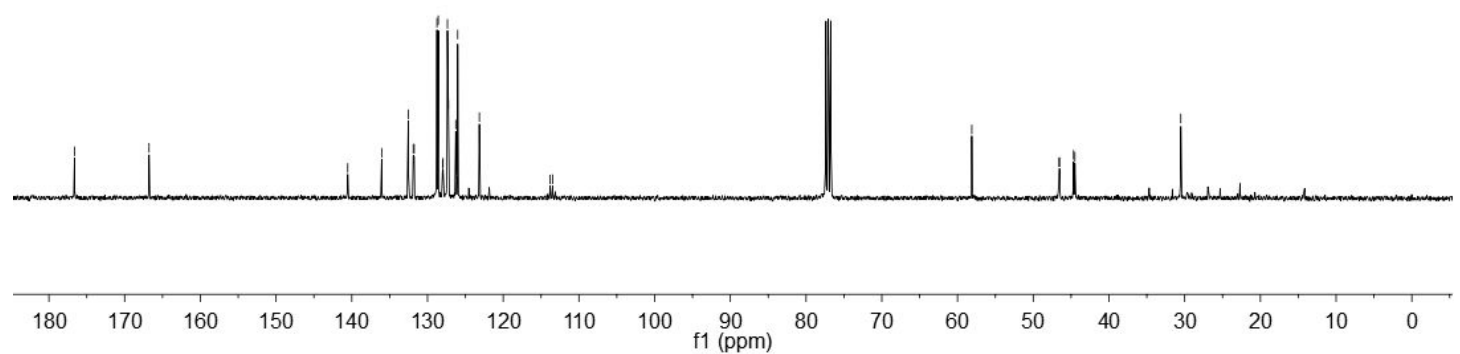

${ }^{1} \mathrm{H},{ }^{19} \mathrm{~F}$ and ${ }^{13} \mathrm{C}$ NMR spectra of $\mathbf{3 p}$

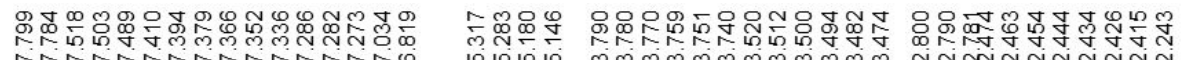

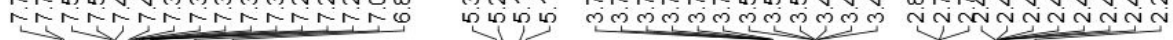

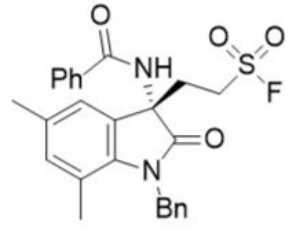

$3 q$

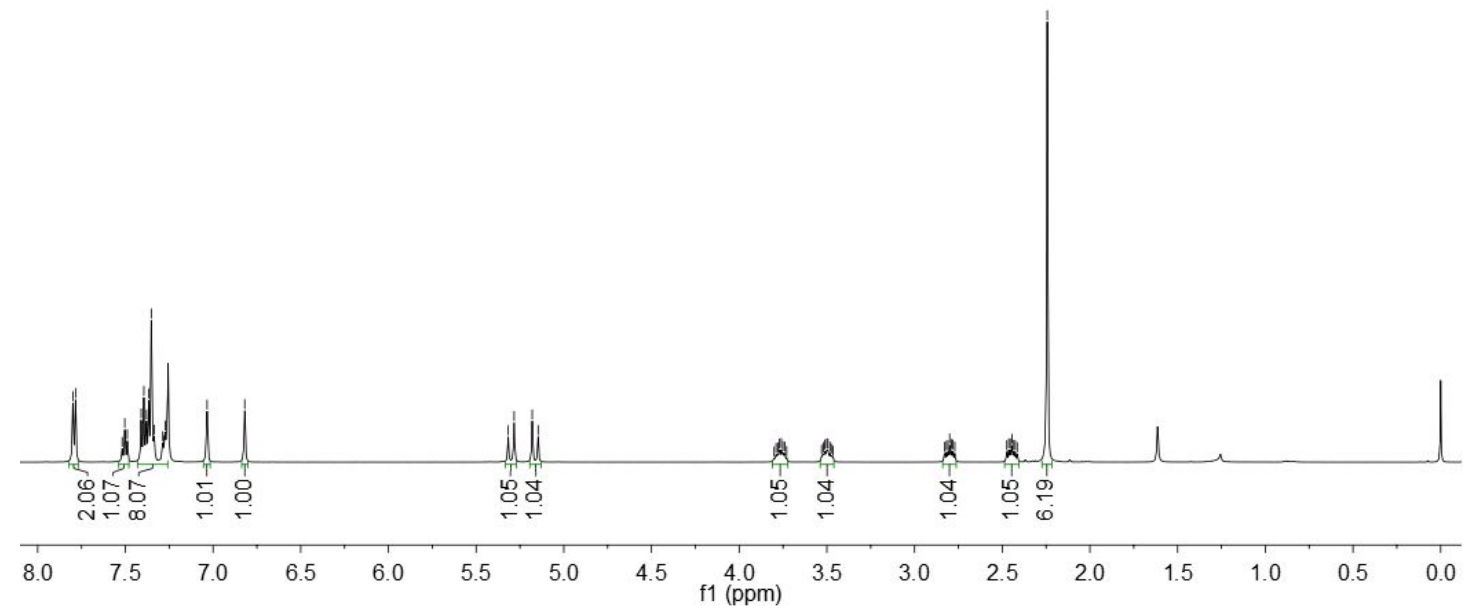




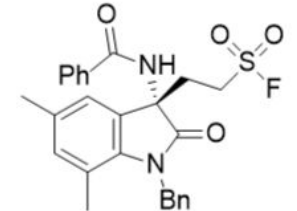

$3 q$

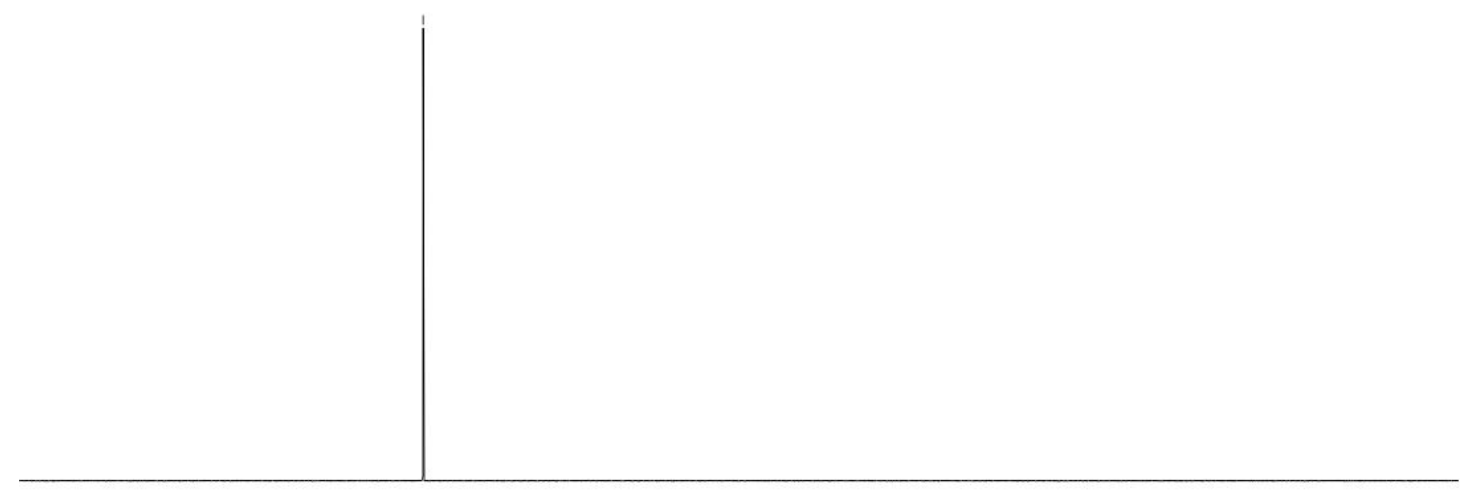

$\begin{array}{lllllllllllllllllllllll}20 & 110 & 100 & 90 & 80 & 70 & 60 & 50 & 40 & 30 & 20 & 10 & 0 & -10 & -20 & -30 & -40 & -50 & -60 & -70 & -80 & -90 & -110\end{array}$

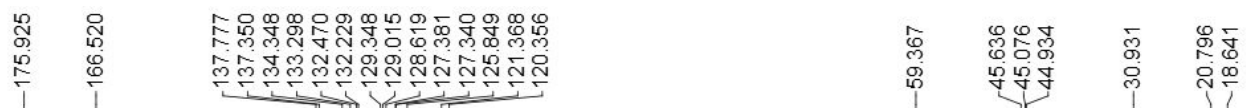

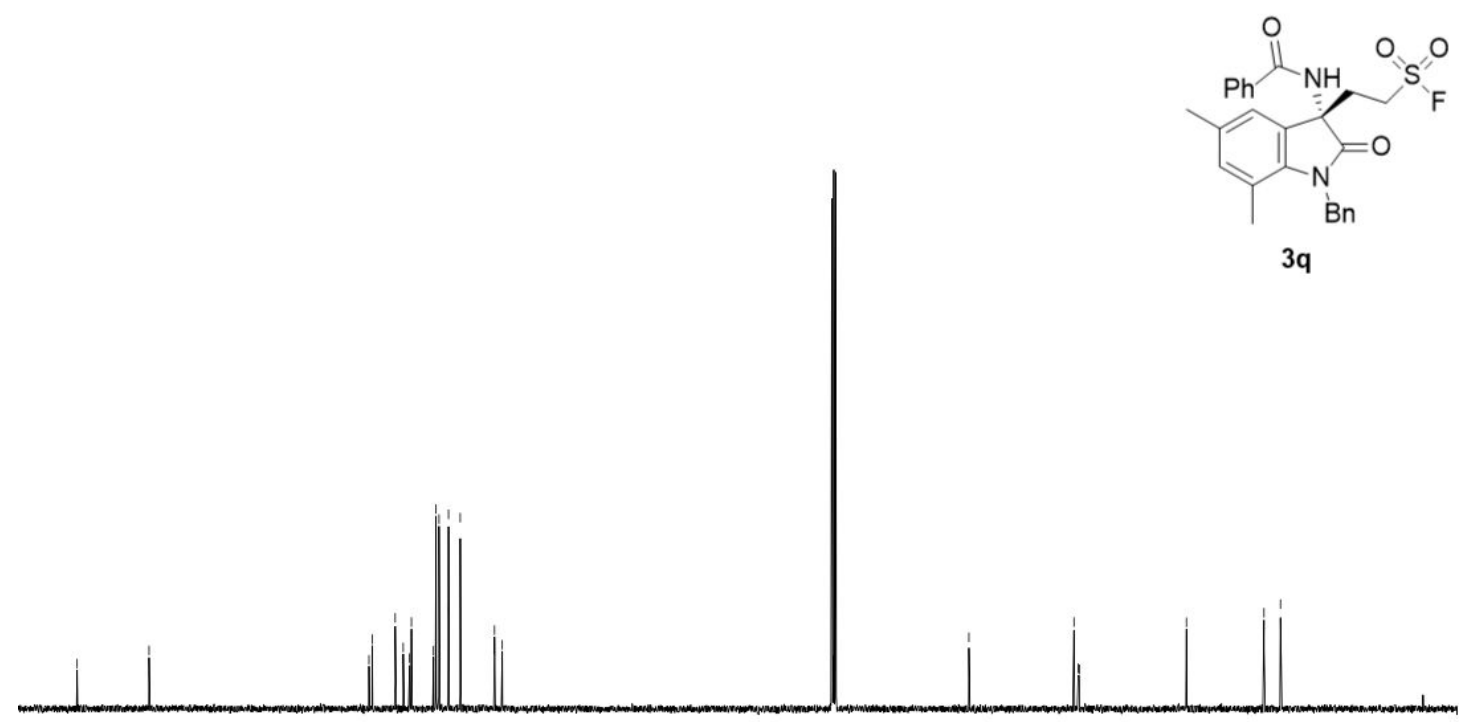



${ }^{1} \mathrm{H},{ }^{19} \mathrm{~F}$ and ${ }^{13} \mathrm{C}$ NMR spectra of $\mathbf{3 q}$ 

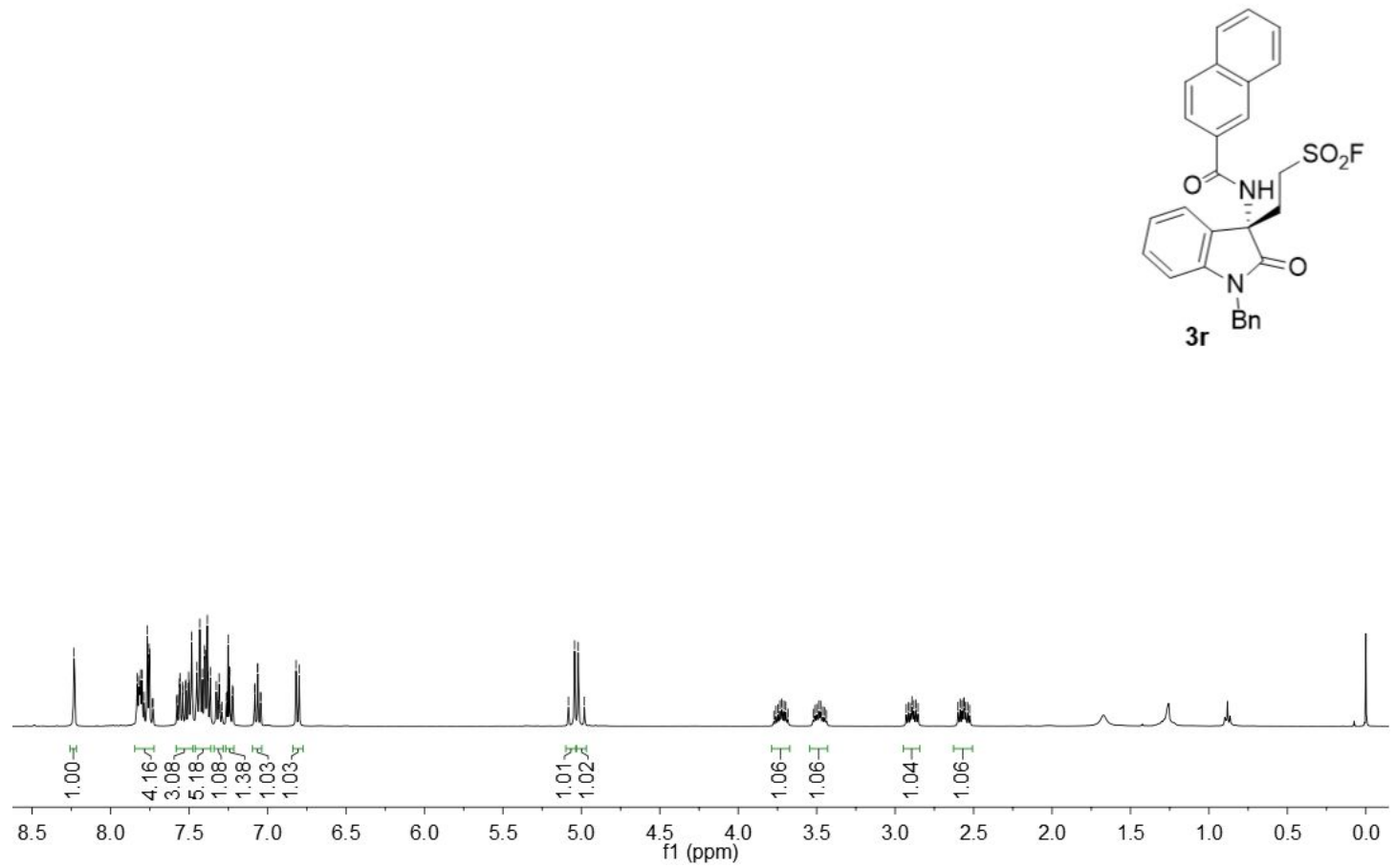

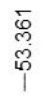

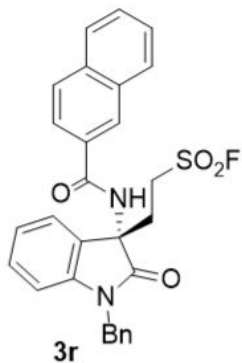

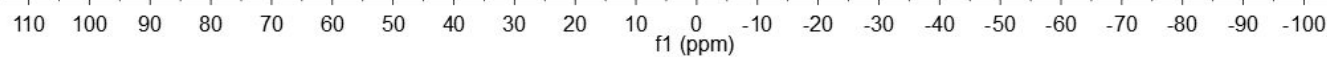



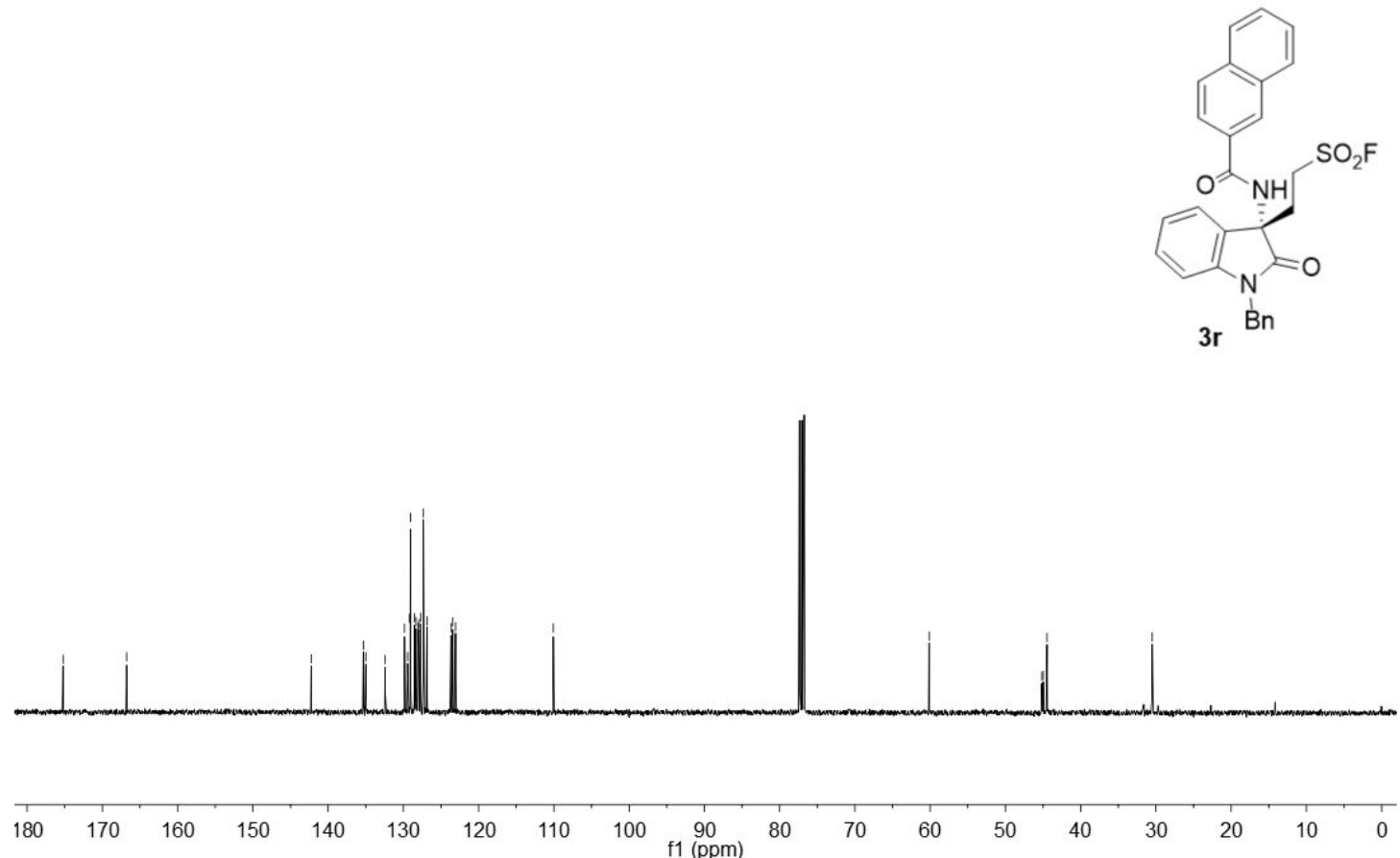

${ }^{1} \mathrm{H},{ }^{19} \mathrm{~F}$ and ${ }^{13} \mathrm{C}$ NMR spectra of $\mathbf{3 r}$

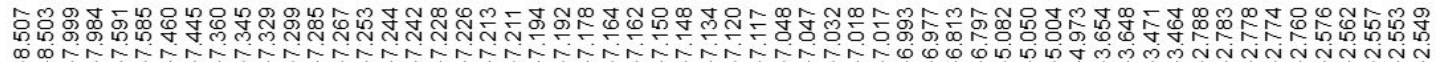

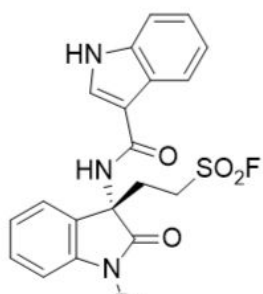

3s

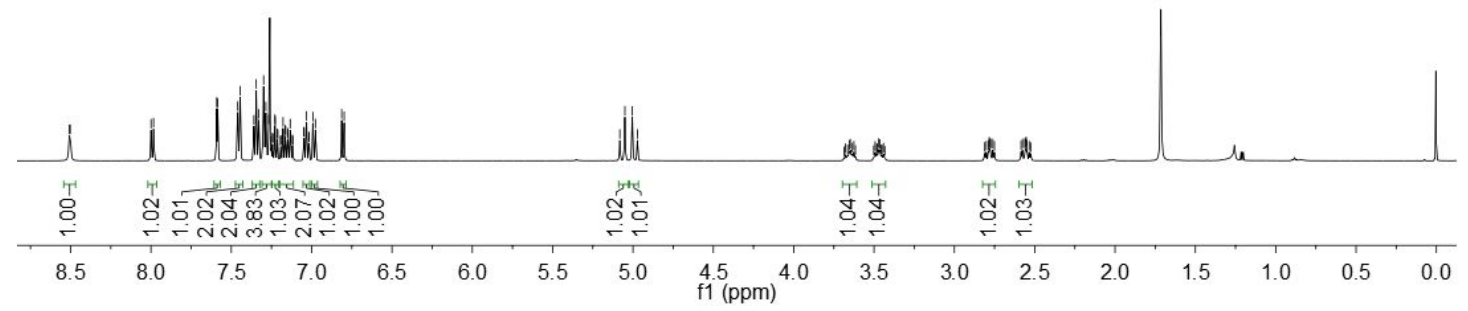


ल

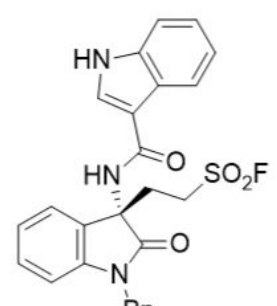

3s $\mathrm{Bn}$

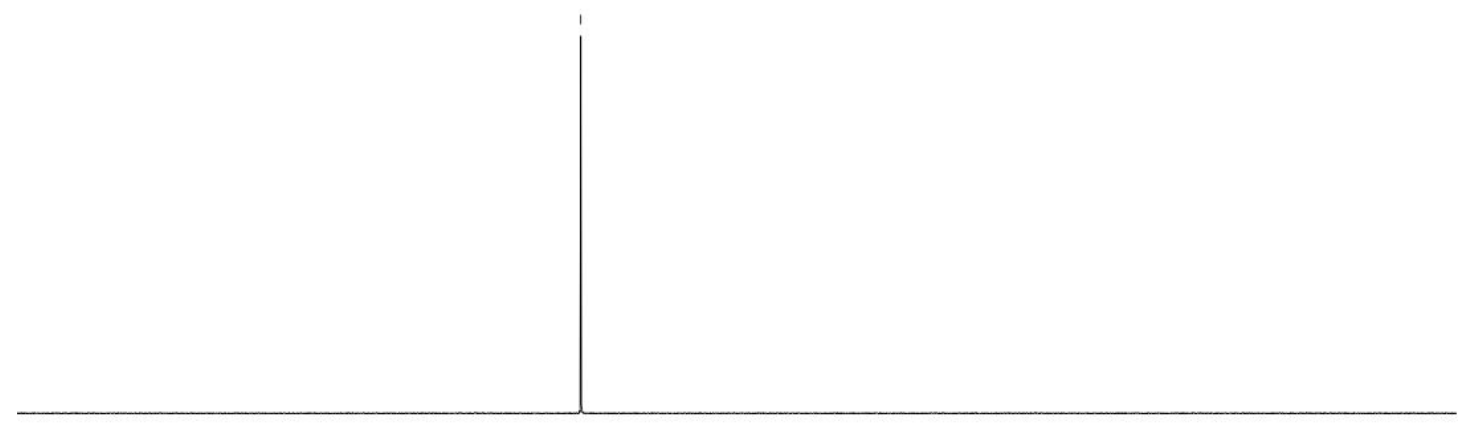

$\begin{array}{llllllllllllllllllllllllll}00 & 95 & 90 & 85 & 80 & 75 & 70 & 65 & 60 & 55 & 50 & 45 & 40 & 35 & 30 & 25 & 20 & 15 & 10 & 5 & 0 & -5 & -10 & -15 & -2\end{array}$

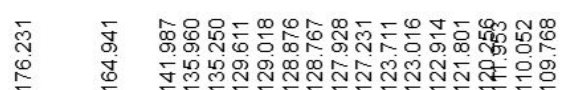

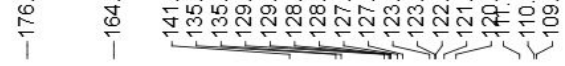

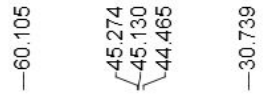

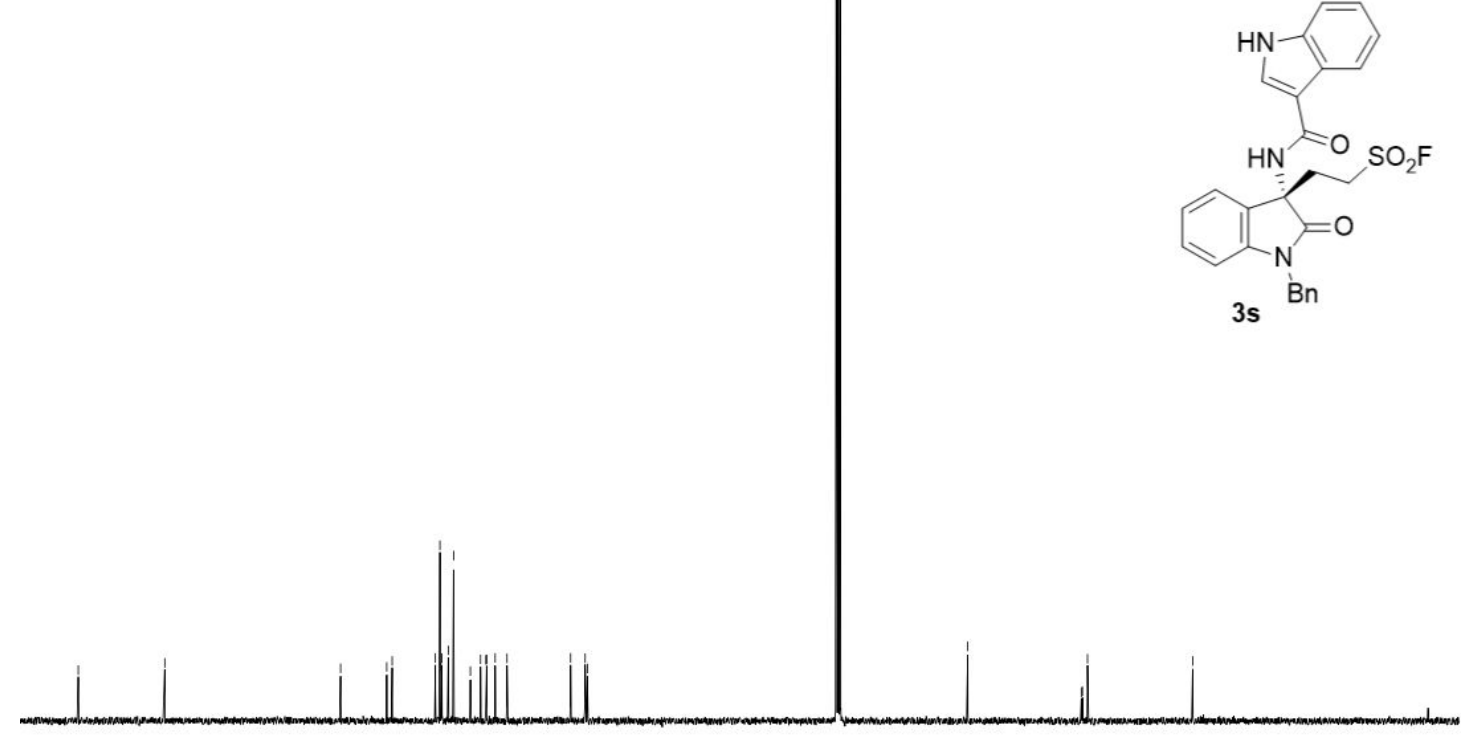

$\begin{array}{lllllllllllllllllll}180 & 170 & 160 & 150 & 140 & 130 & 120 & 110 & 100 & \begin{array}{c}90 \\ \mathrm{f} 1(\mathrm{ppm})\end{array} & 80 & 70 & 60 & 50 & 40 & 30 & 20 & 10 & 0\end{array}$

${ }^{1} \mathrm{H},{ }^{19} \mathrm{~F}$ and ${ }^{13} \mathrm{C}$ NMR spectra of $\mathbf{3 s}$ 

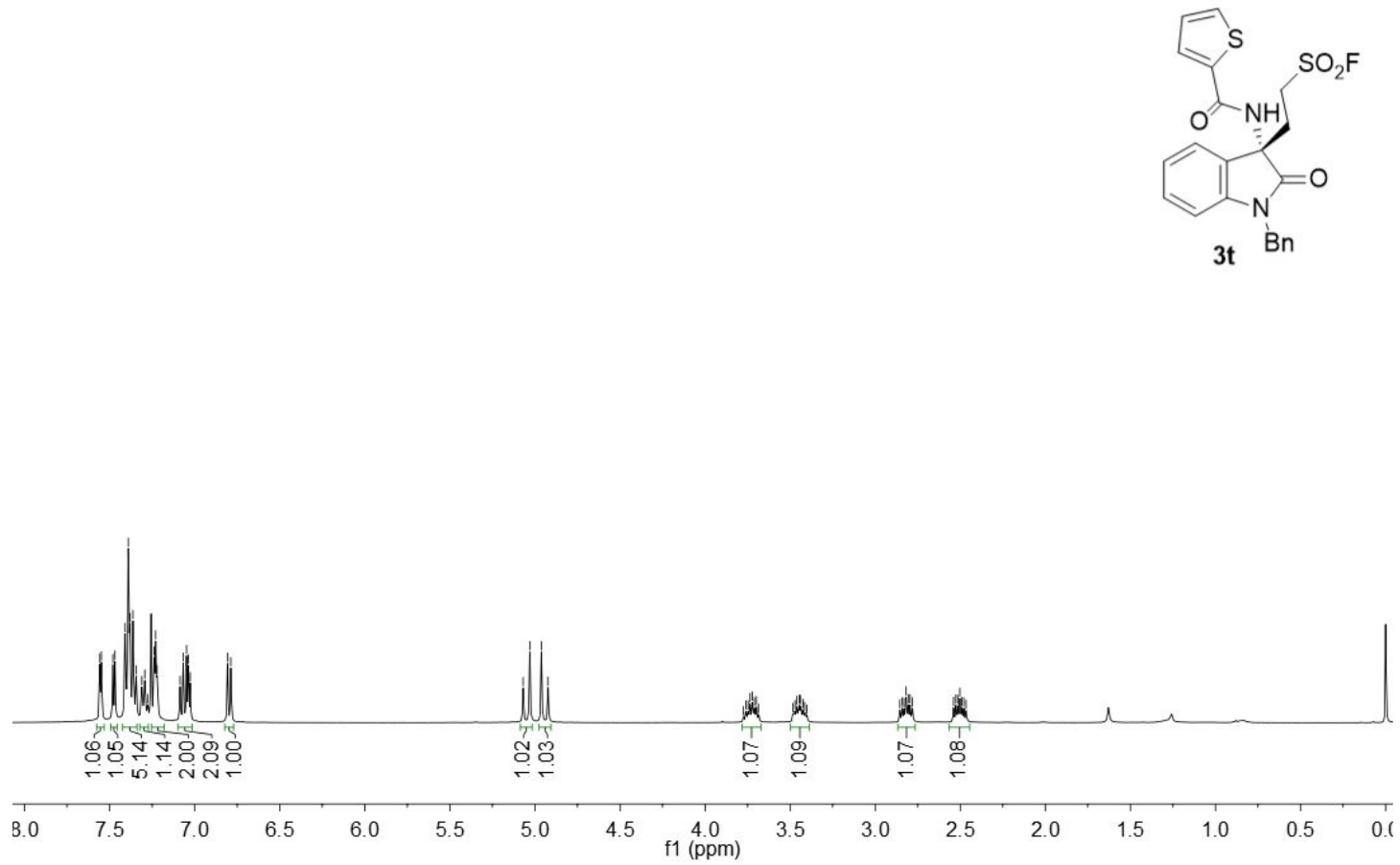

$\underset{\substack{\text { ले } \\ \text { in }}}{i}$

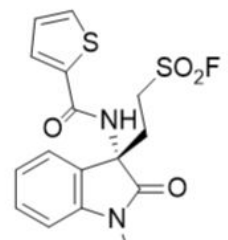

3t Bn

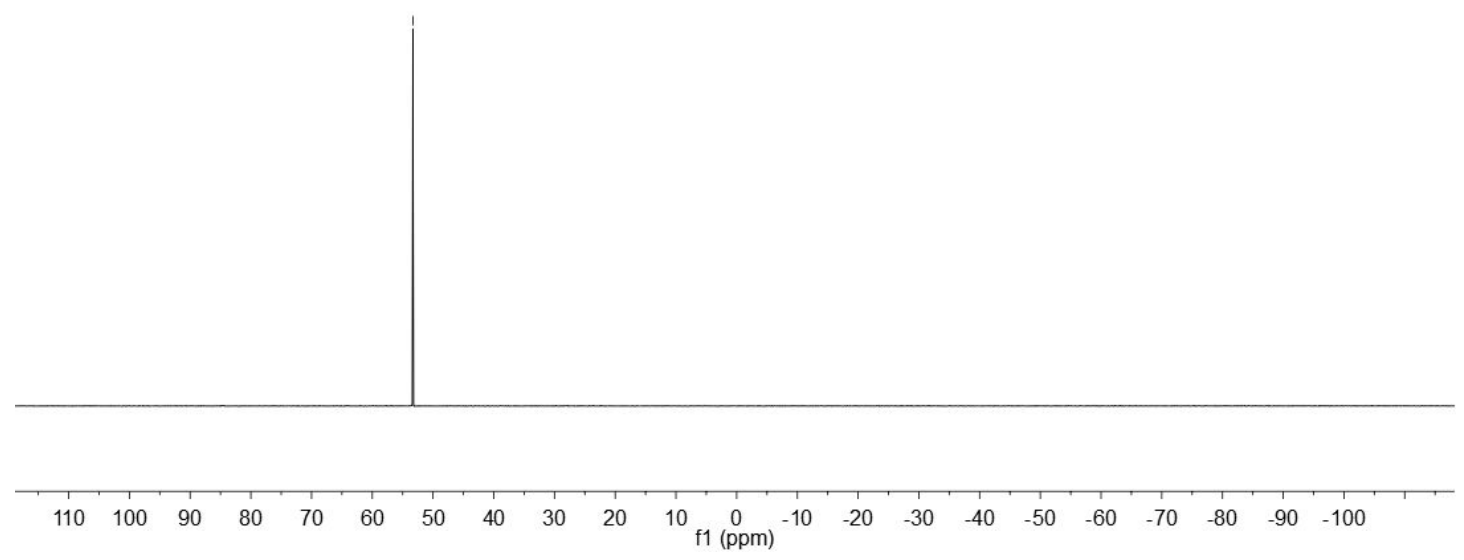



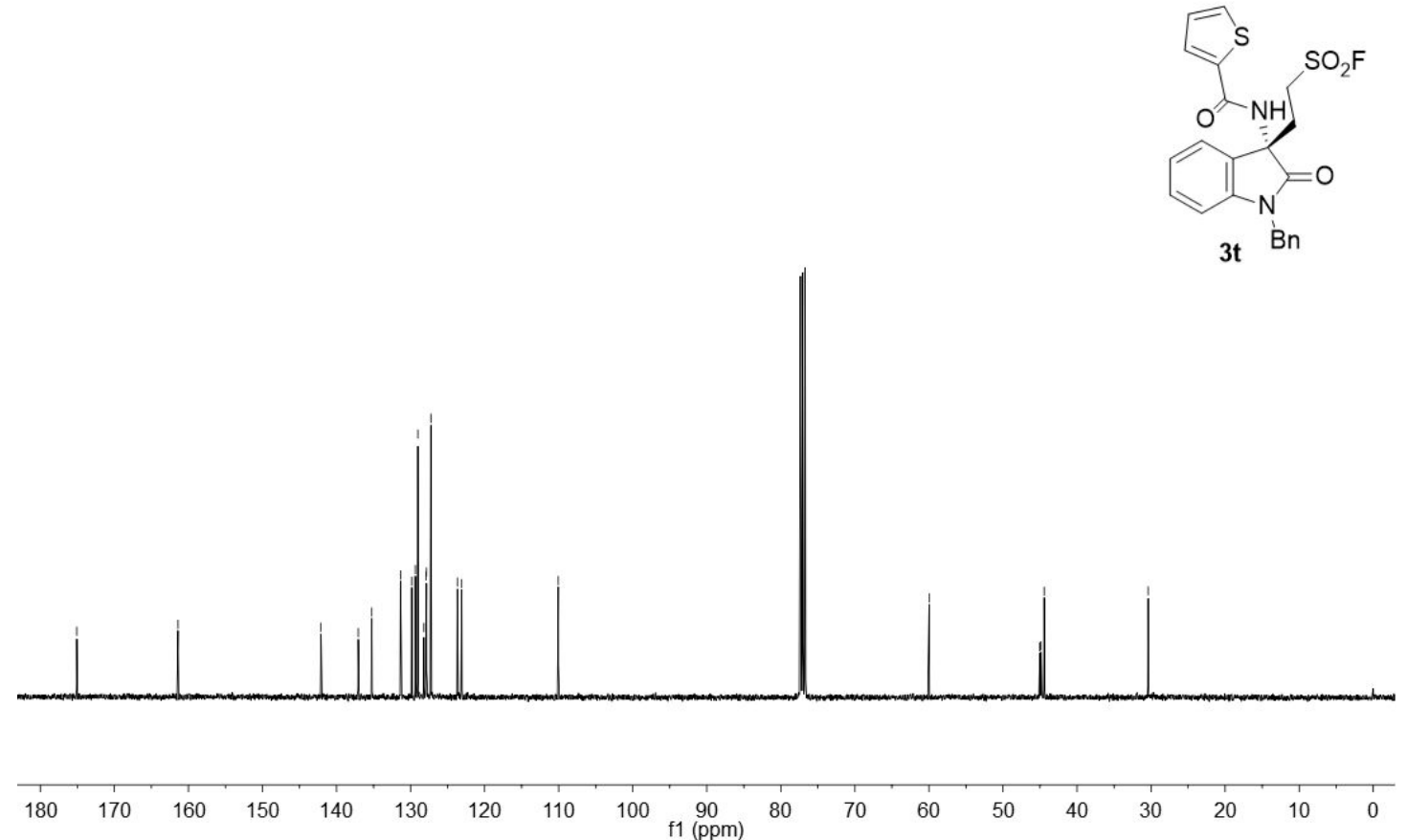

${ }^{1} \mathrm{H},{ }^{19} \mathrm{~F}$ and ${ }^{13} \mathrm{C}$ NMR spectra of $\mathbf{3 t}$

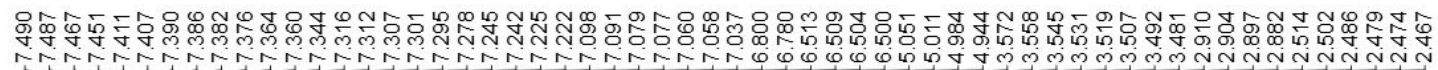
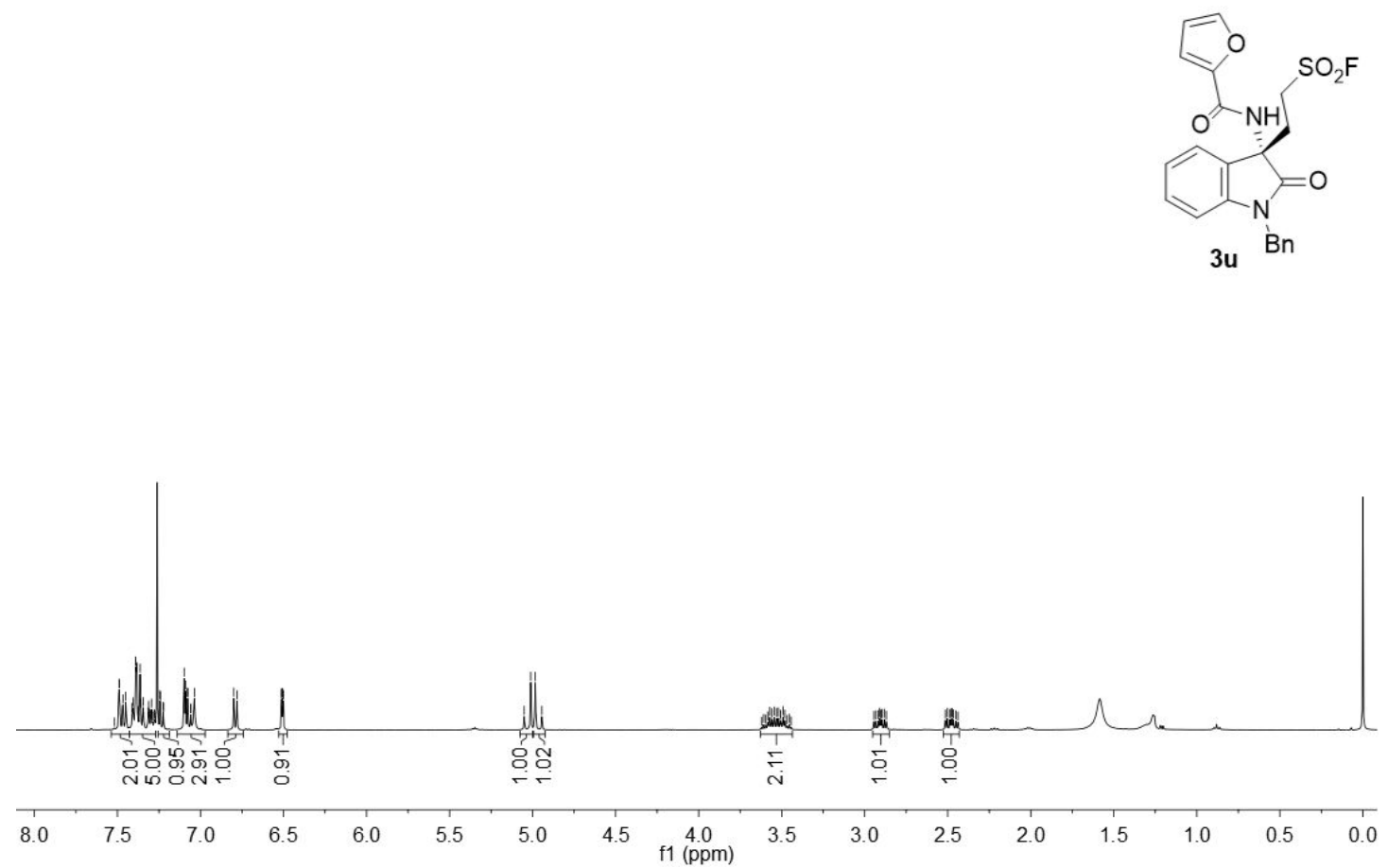

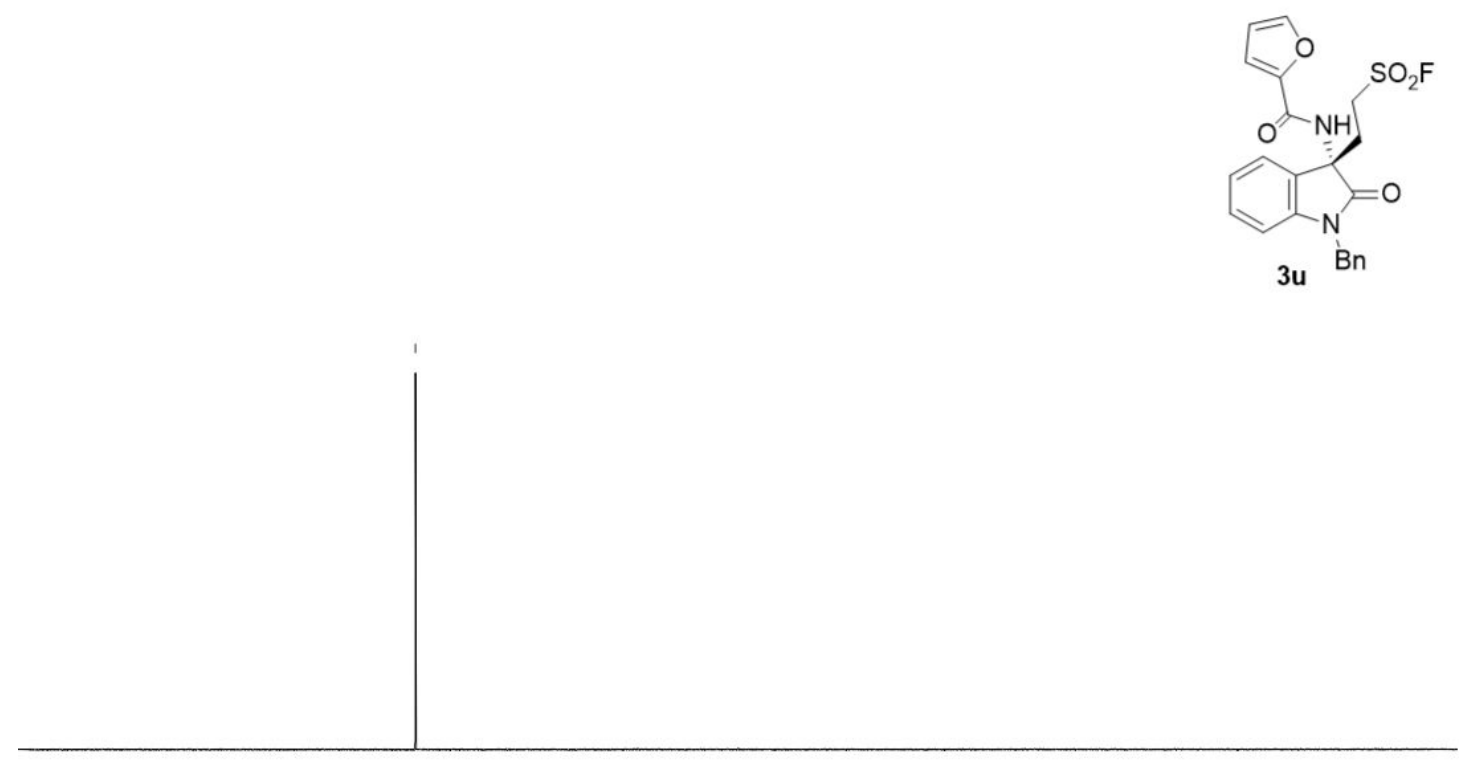

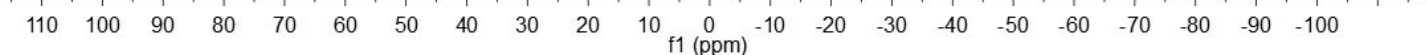

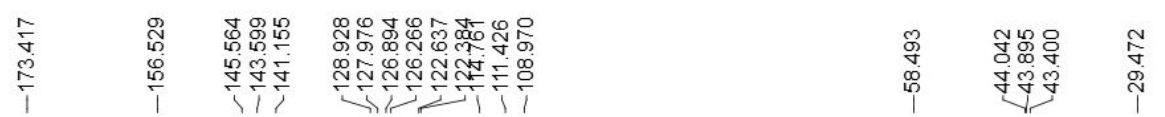

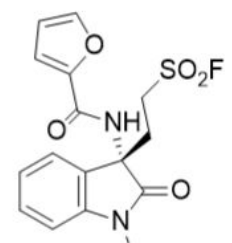

$3 \mathrm{u} \mathrm{Bn}$

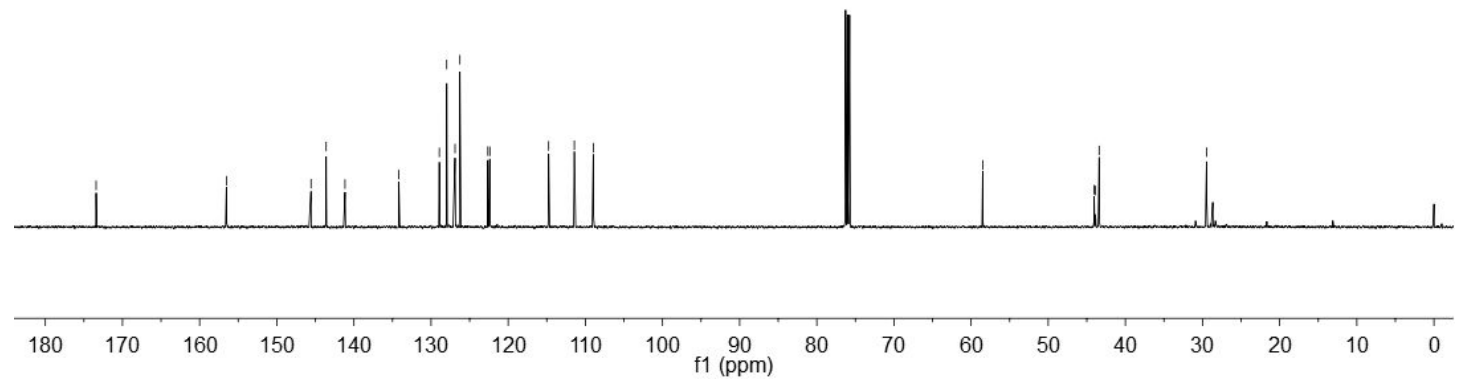

${ }^{1} \mathrm{H},{ }^{19} \mathrm{~F}$ and ${ }^{13} \mathrm{C}$ NMR spectra of $\mathbf{3 u}$ 


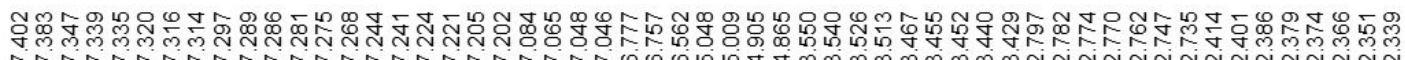

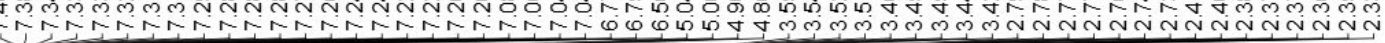

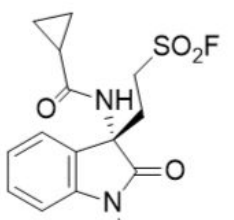

$3 v^{B n}$

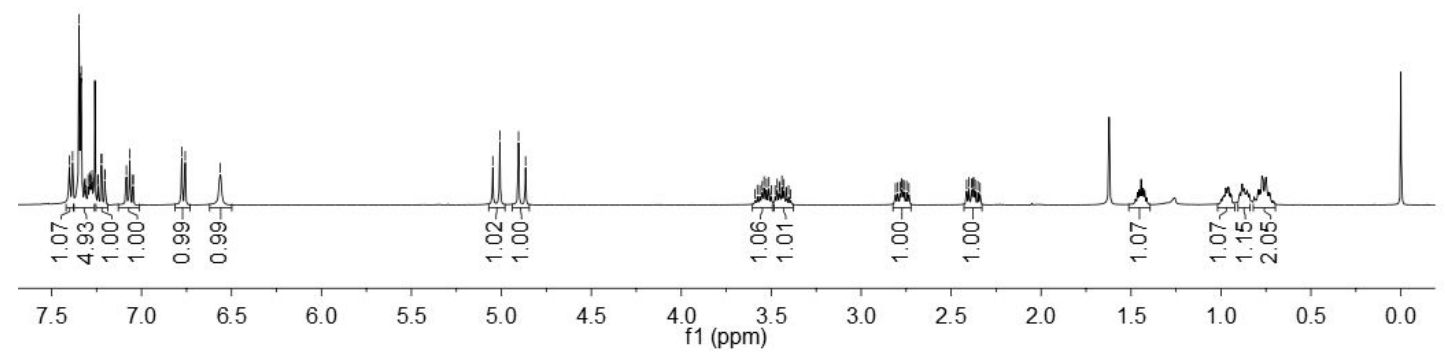

$\underset{\substack{0 \\ N}}{0}$

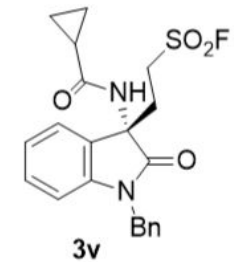

$\begin{array}{llllllllllllllllllllll}110 & 100 & 90 & 80 & 70 & 60 & 50 & 40 & 30 & 20 & 10 & 0 & 0 \\ \mathrm{f} 1(\mathrm{ppm}) & -10 & -20 & -30 & -40 & -50 & -60 & -70 & -80 & -90 & -100\end{array}$ 

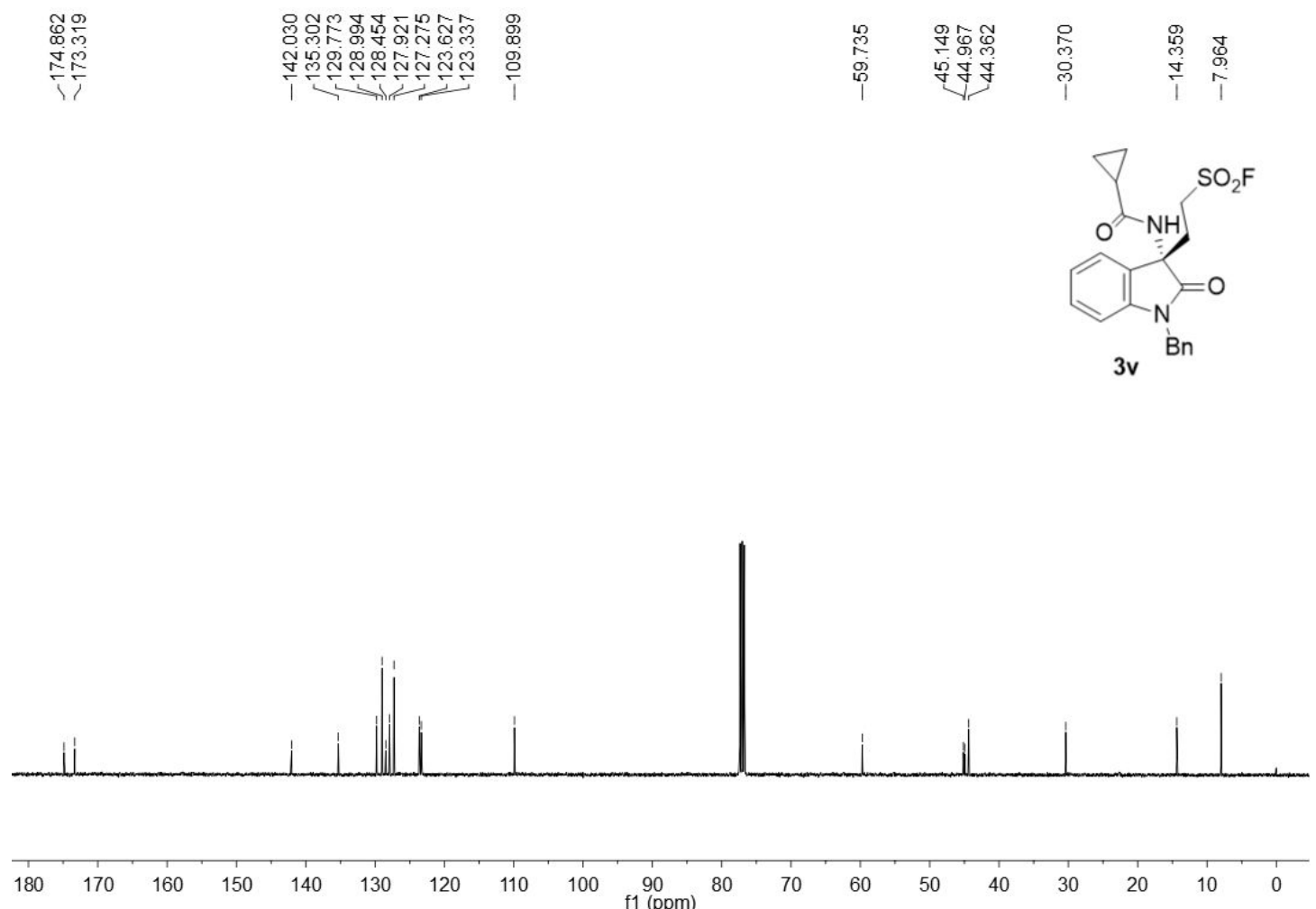

${ }^{1} \mathrm{H},{ }^{19} \mathrm{~F}$ and ${ }^{13} \mathrm{C}$ NMR spectra of $\mathbf{3 v}$

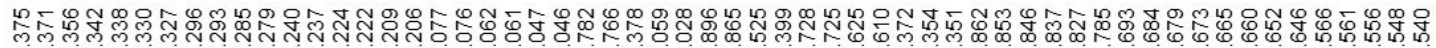

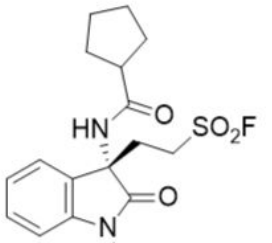

3w Bn

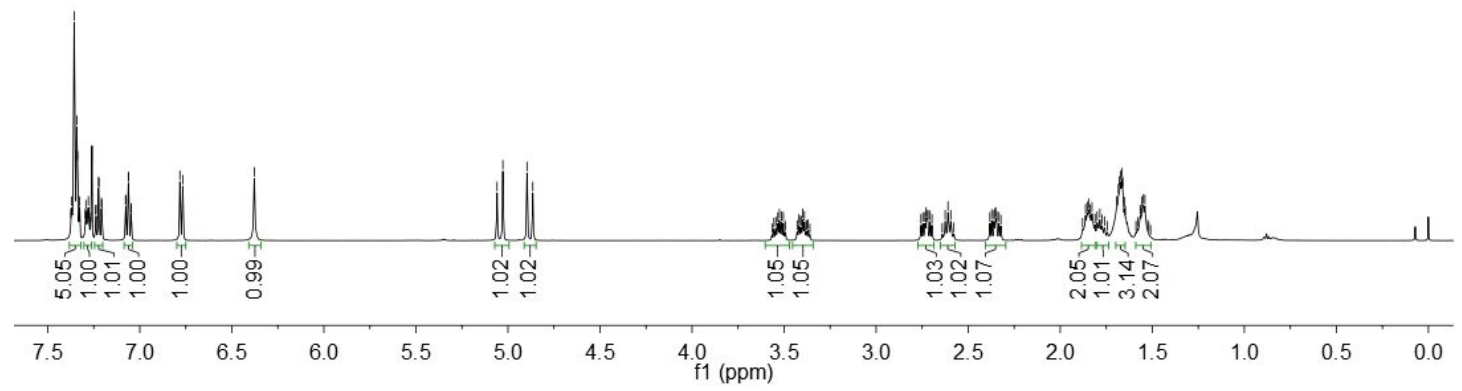




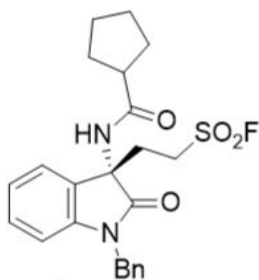

$3 w$

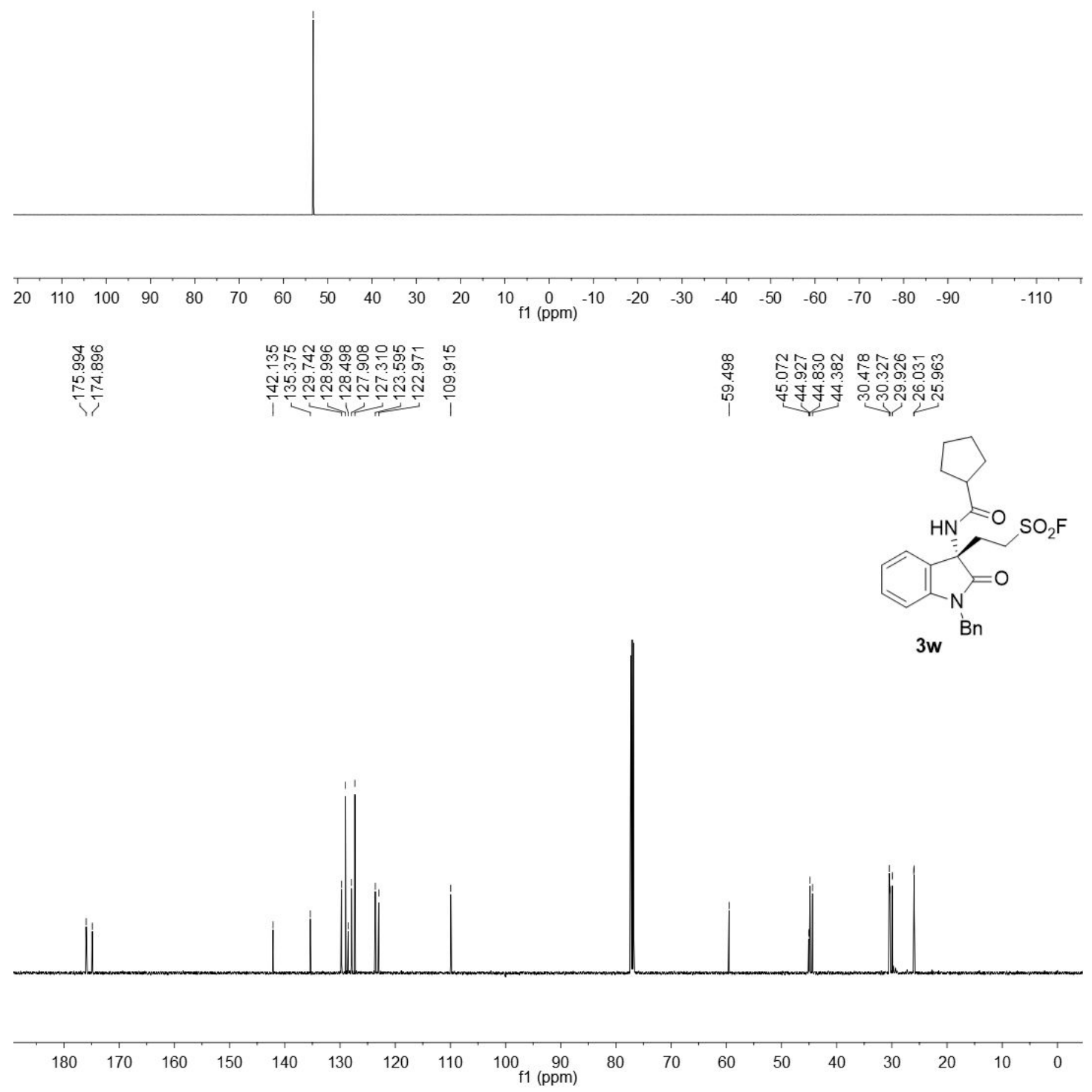

${ }^{1} \mathrm{H},{ }^{19} \mathrm{~F}$ and ${ }^{13} \mathrm{C}$ NMR spectra of $\mathbf{3} \mathbf{w}$ 


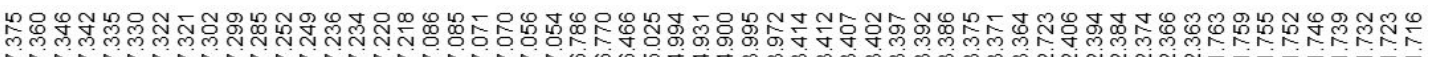

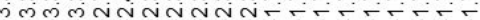

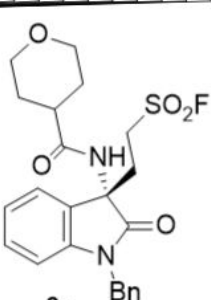

$3 x$

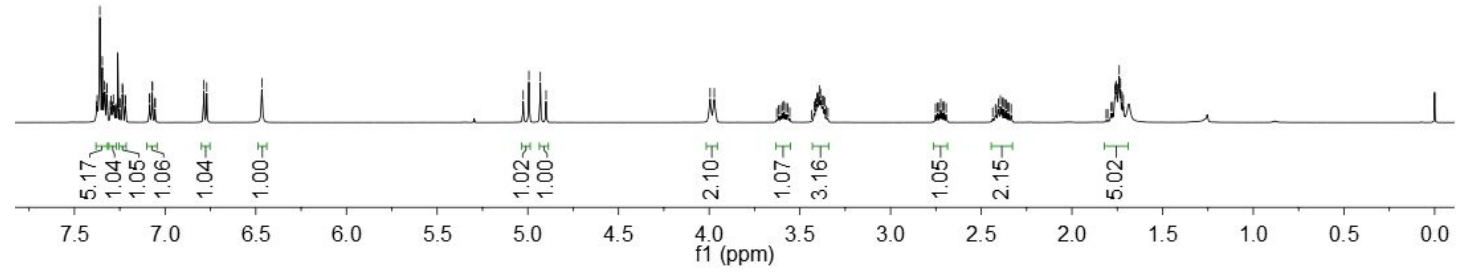

חָ

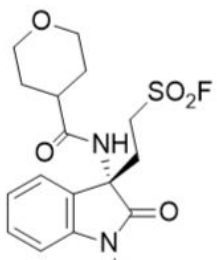

$3 \mathbf{x}$

$\mathrm{Bn}$

$\begin{array}{lllllllllllllllllllll}0 & 110 & 100 & 90 & 80 & 70 & 60 & 50 & 40 & 30 & 20 & 10 & 0 \\ \mathrm{f} 1(\mathrm{ppm}) & -10 & -20 & -30 & -40 & -50 & -60 & -70 & -80 & -90 & -110\end{array}$ 


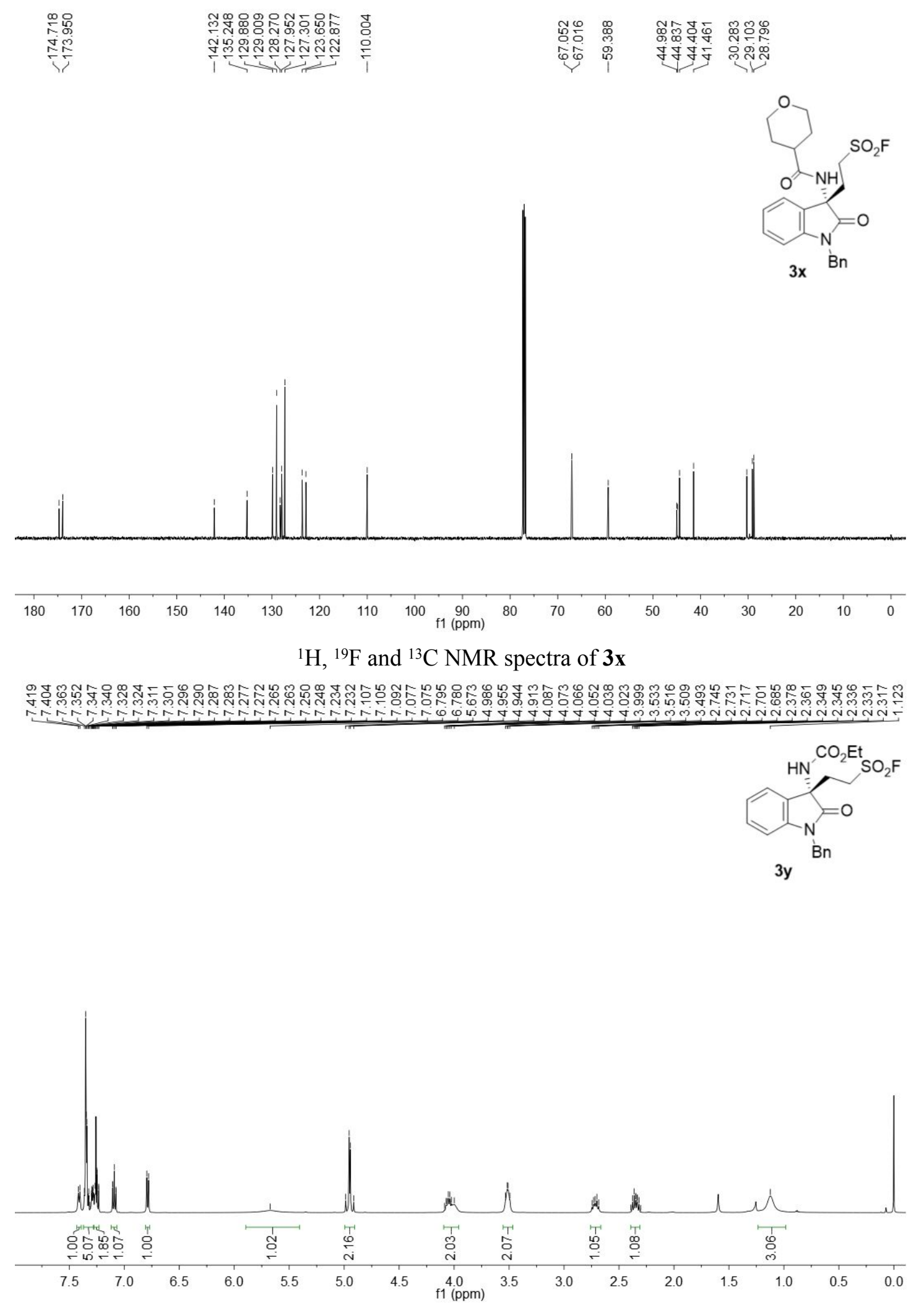




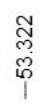
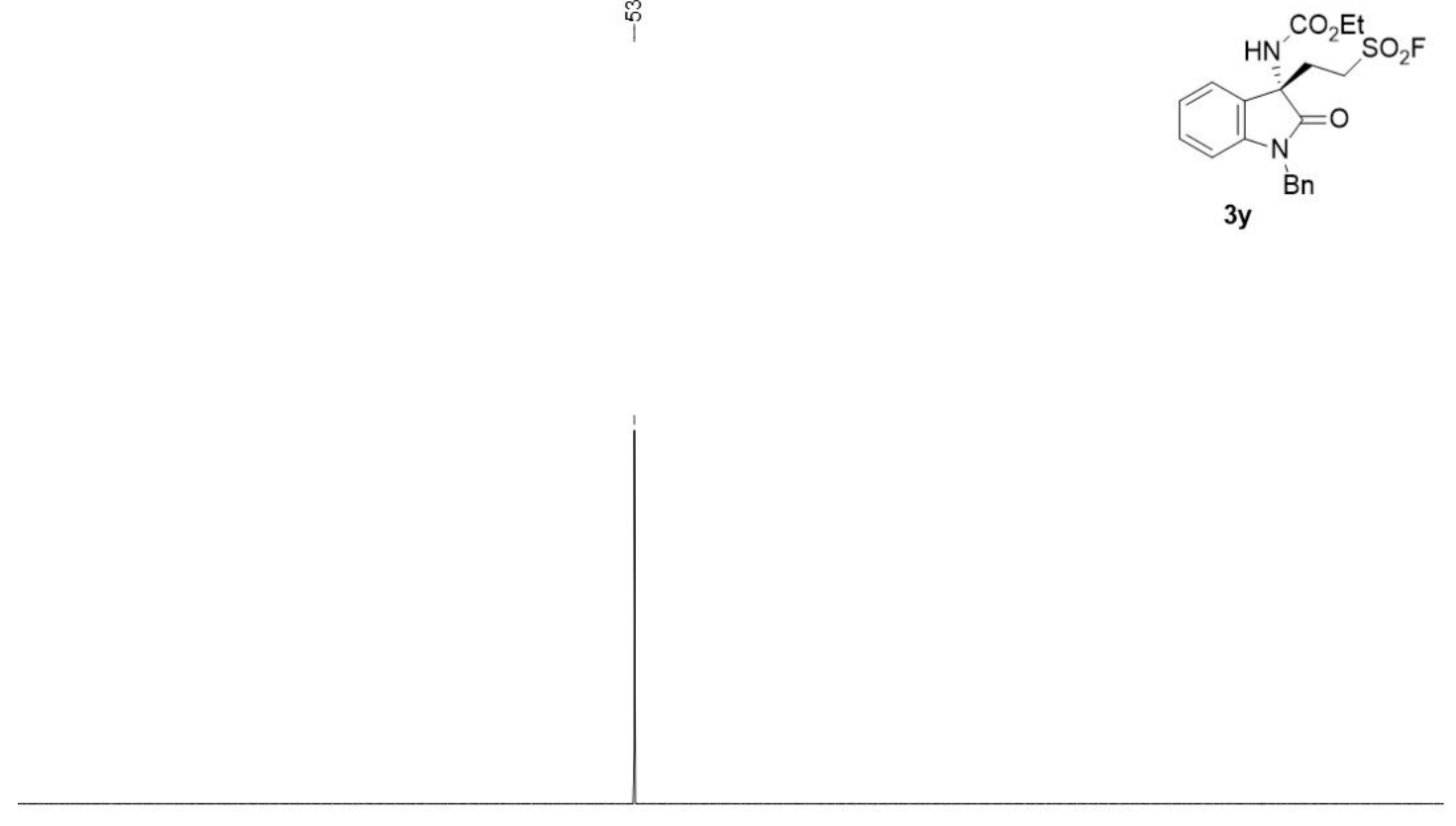

$\begin{array}{lllllllllllllllllllllllllllllllll}05 & 100 & 95 & 90 & 85 & 80 & 75 & 70 & 65 & 60 & 55 & 50 & \begin{array}{l}45 \\ \mathrm{fpm})\end{array} & 40 & 35 & 30 & 25 & 20 & 15 & 10 & 5 & 0 & -5 & -10 & -1\end{array}$

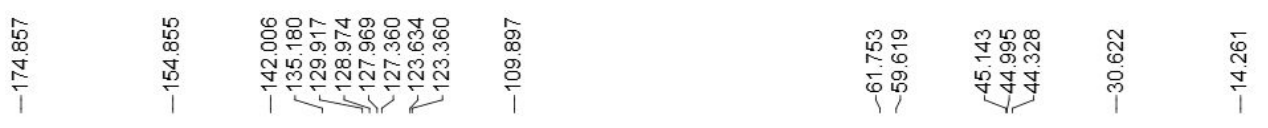



${ }^{1} \mathrm{H},{ }^{19} \mathrm{~F}$ and ${ }^{13} \mathrm{C}$ NMR spectra of $\mathbf{3 y}$ 


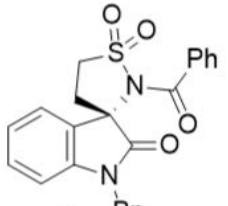

5a Bn

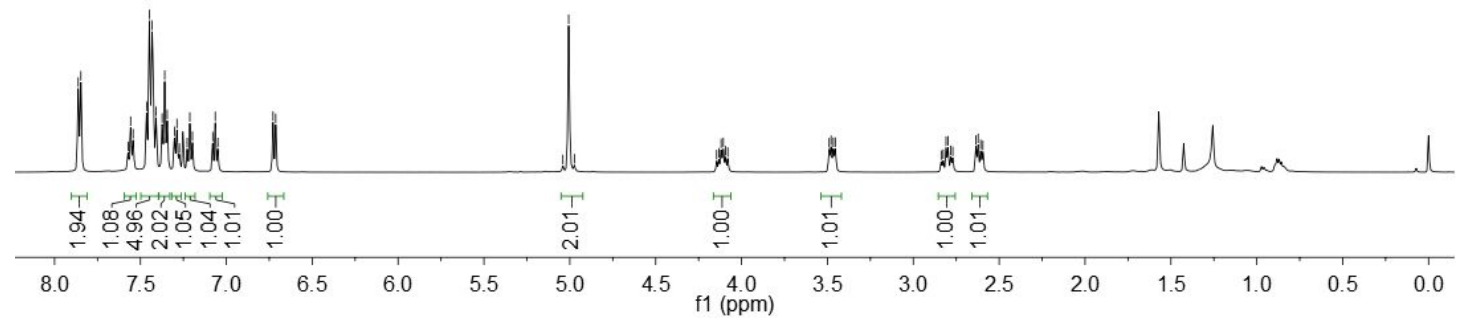

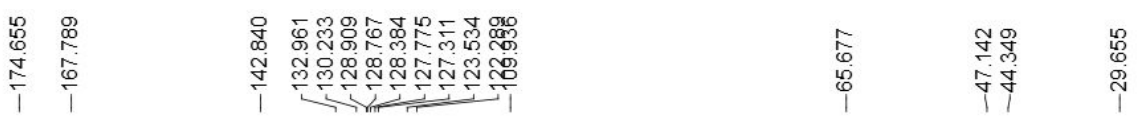

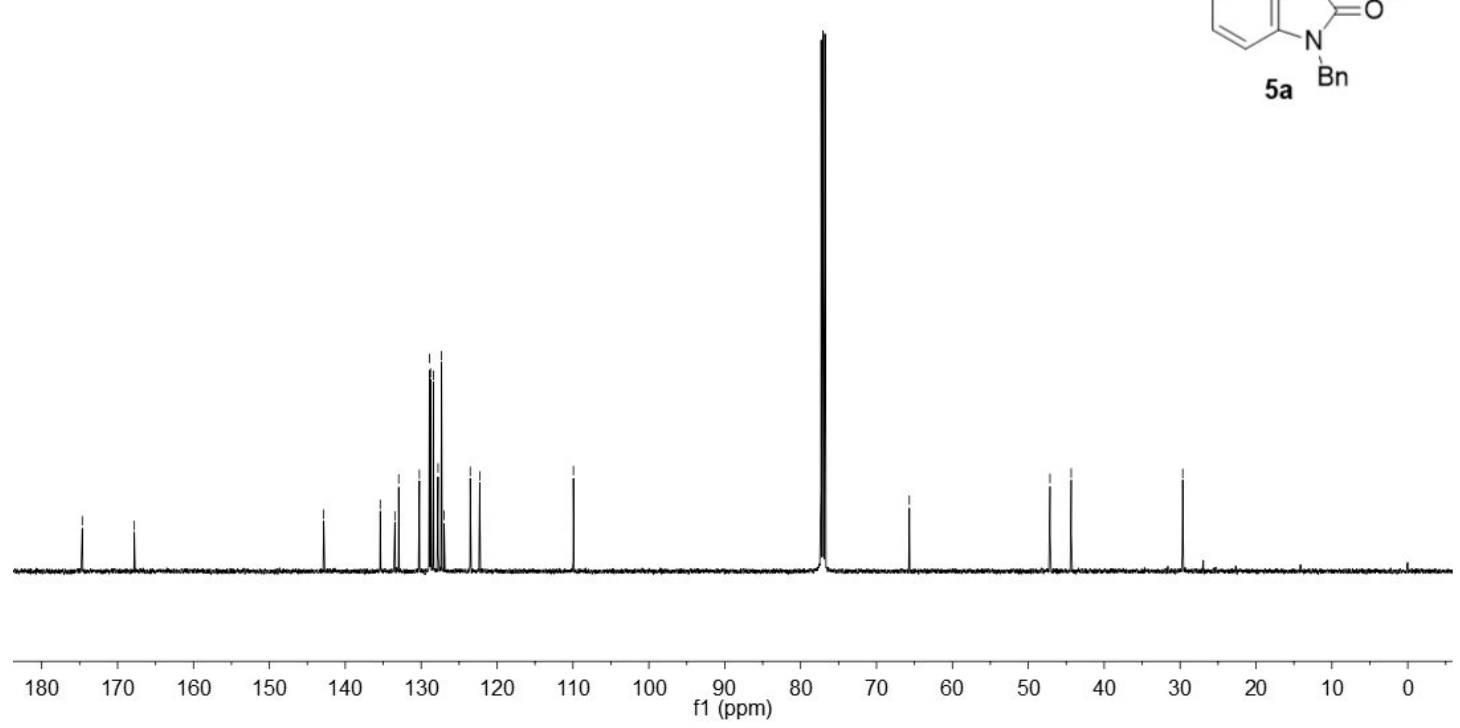

${ }^{1} \mathrm{H}$ and ${ }^{13} \mathrm{C}$ NMR spectra of $\mathbf{5 a}$ 

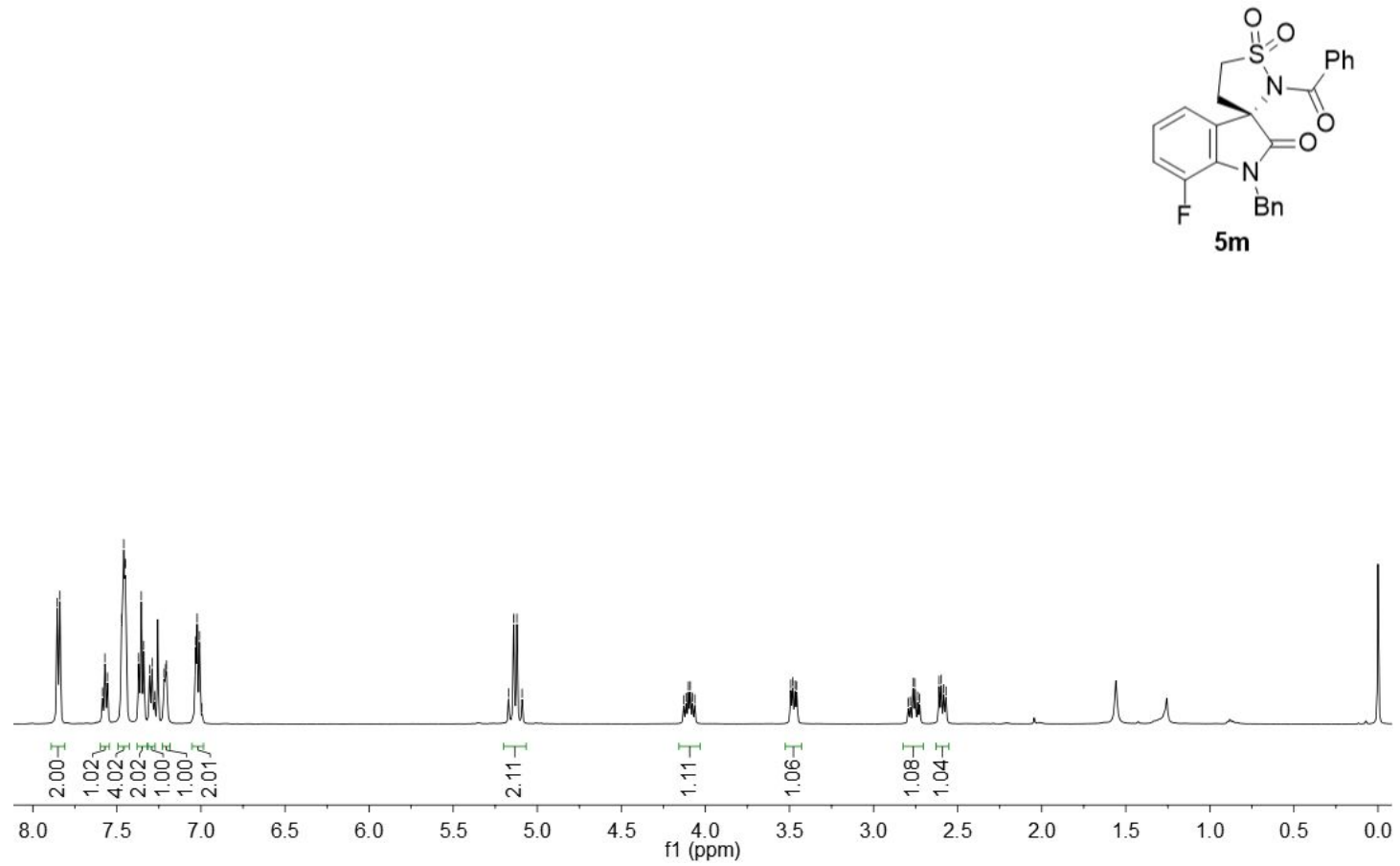

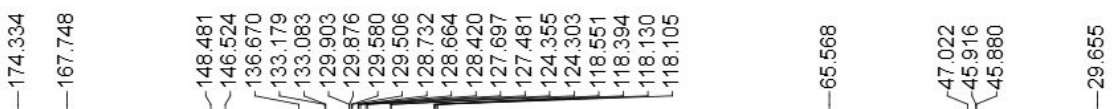

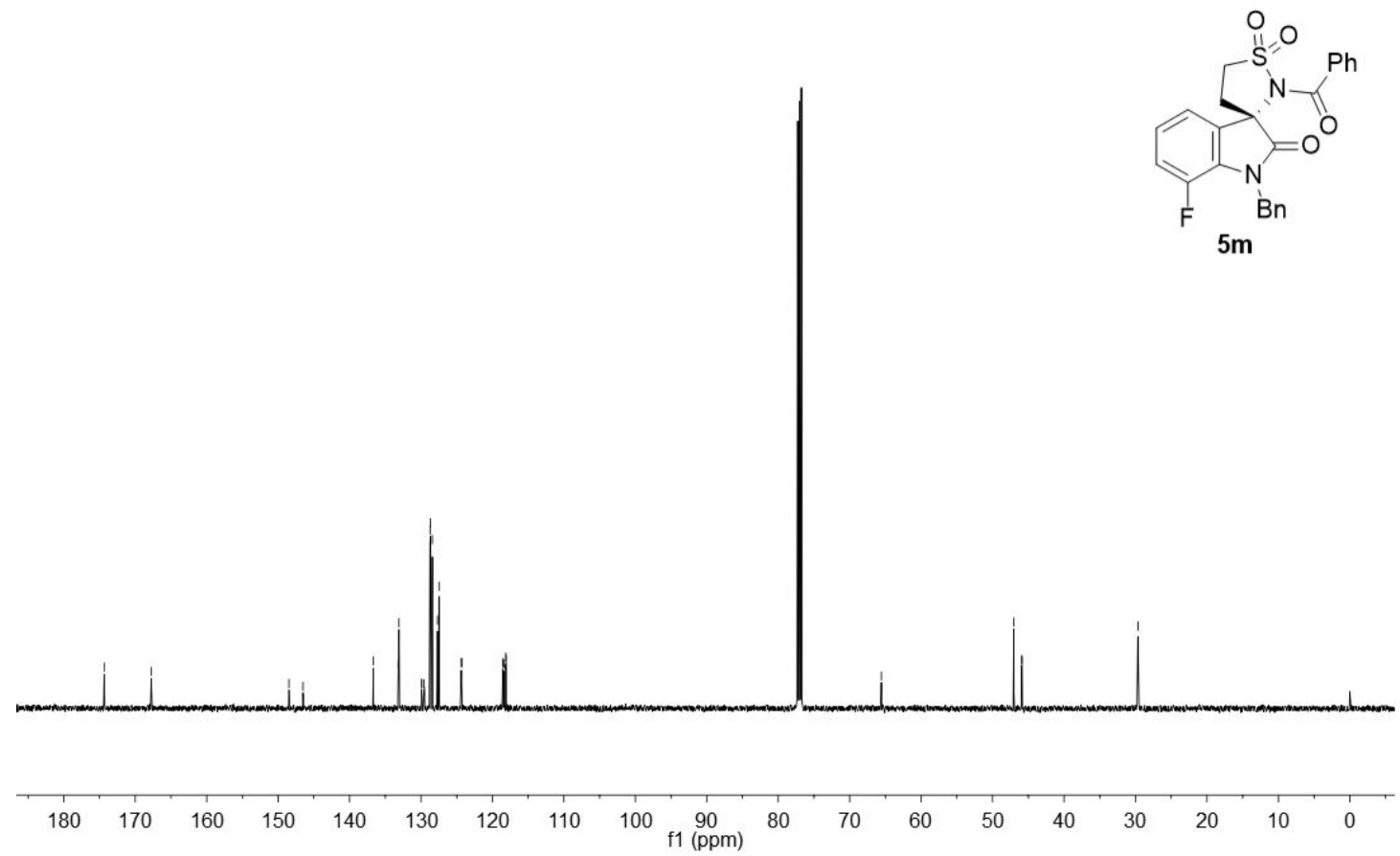

${ }^{1} \mathrm{H}$ and ${ }^{13} \mathrm{C}$ NMR spectra of $\mathbf{5 m}$ 

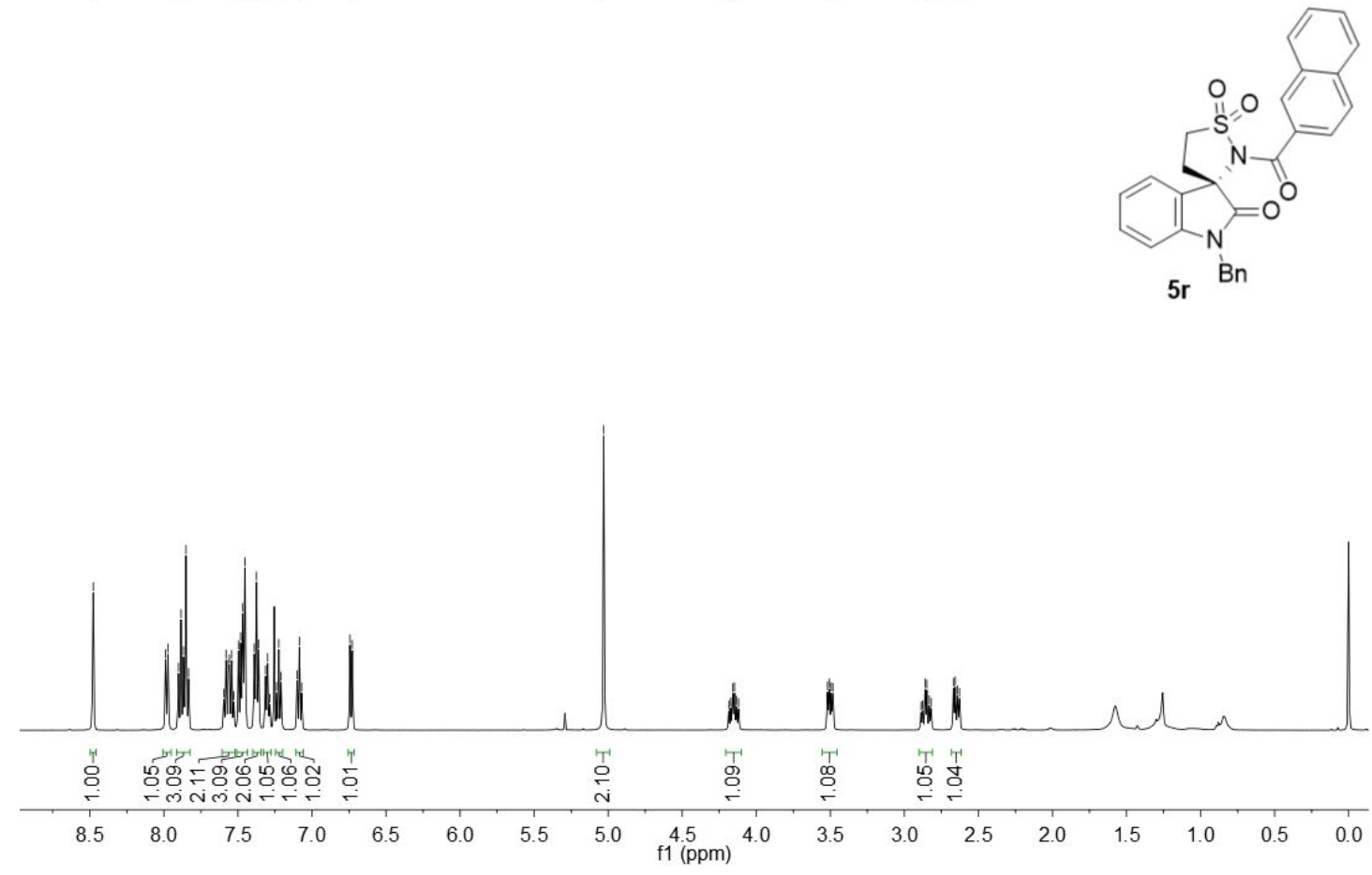

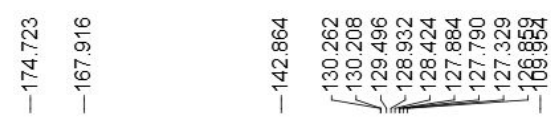

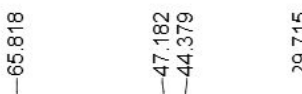

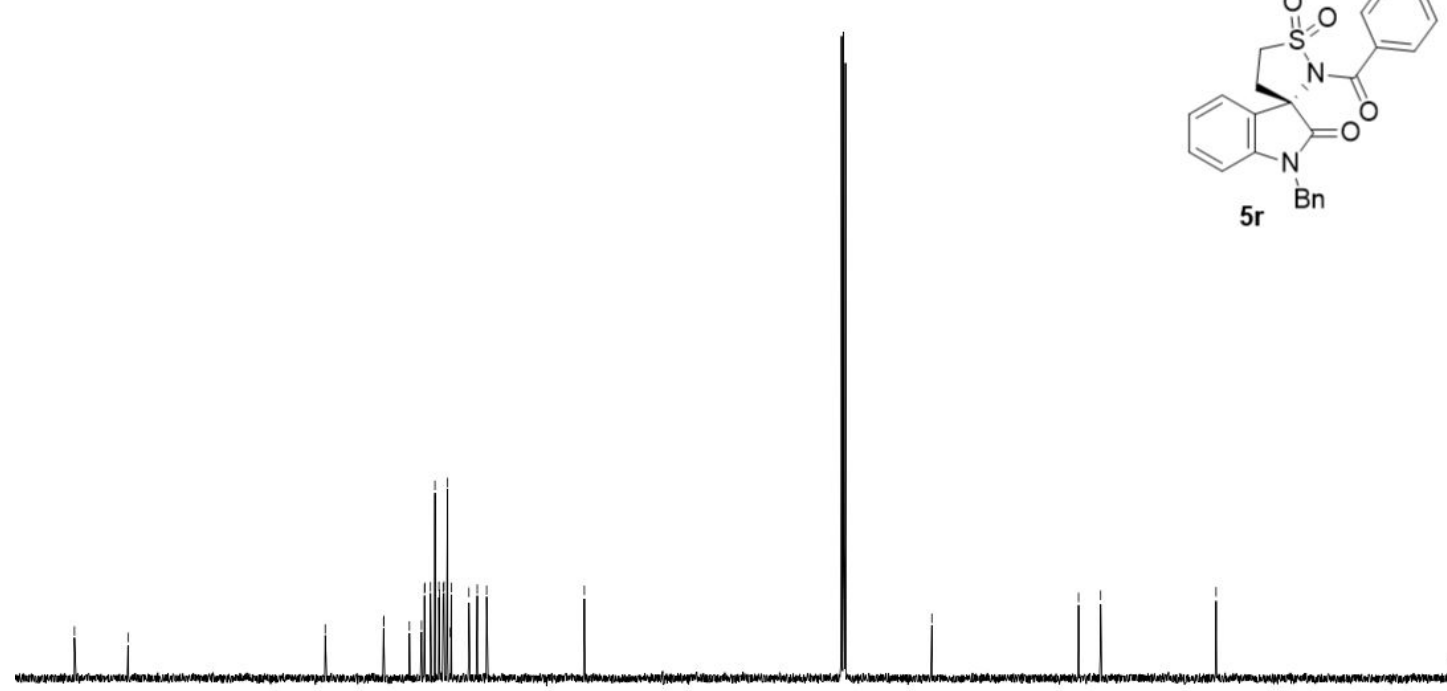

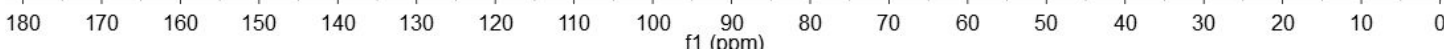

${ }^{1} \mathrm{H}$ and ${ }^{13} \mathrm{C}$ NMR spectra of $\mathbf{5 r}$ 

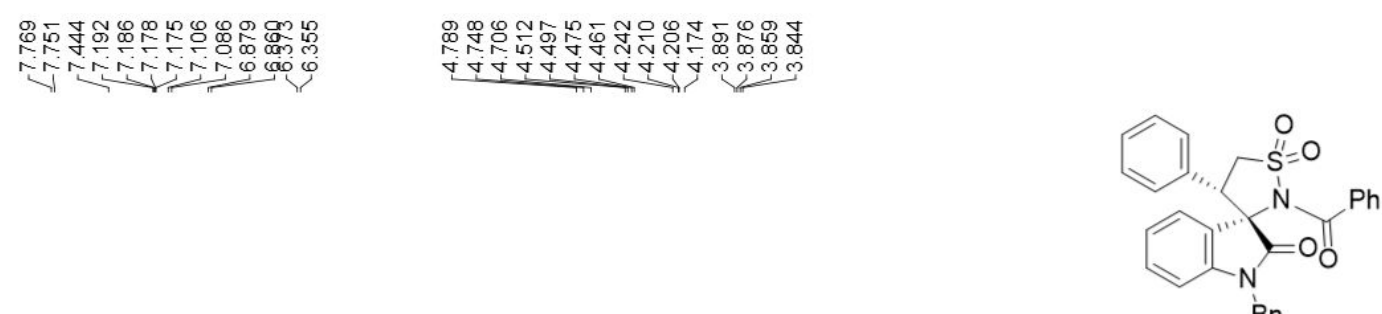

6ab ${ }^{\mathrm{Bn}}$

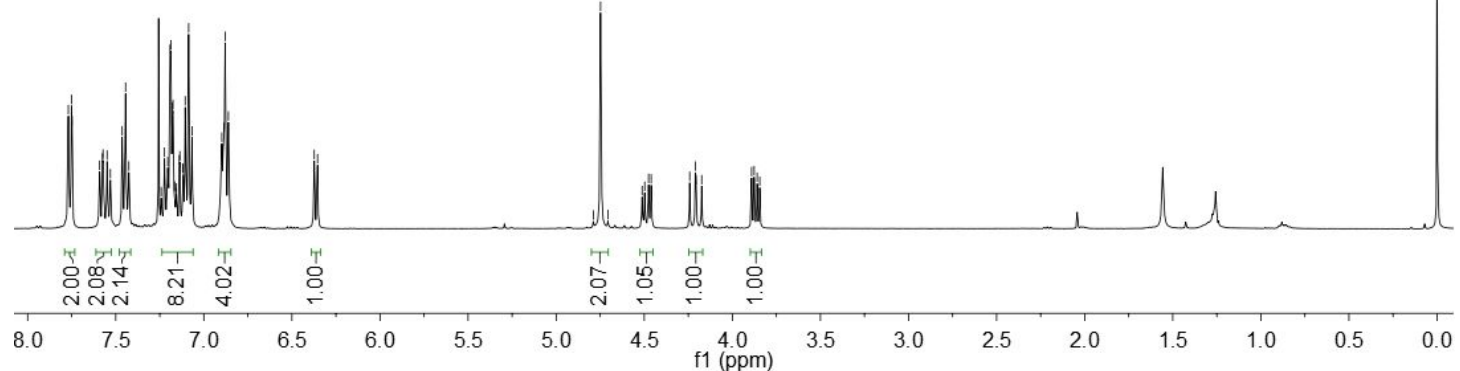

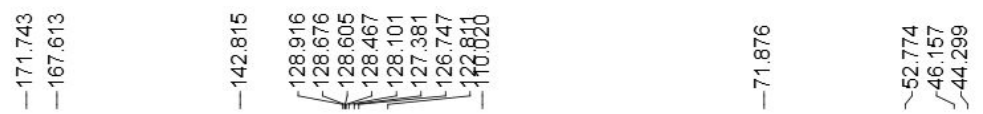

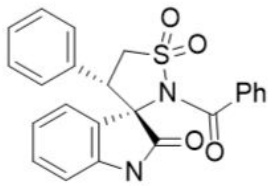

$6 \mathrm{ab}^{\mathrm{Bn}}$

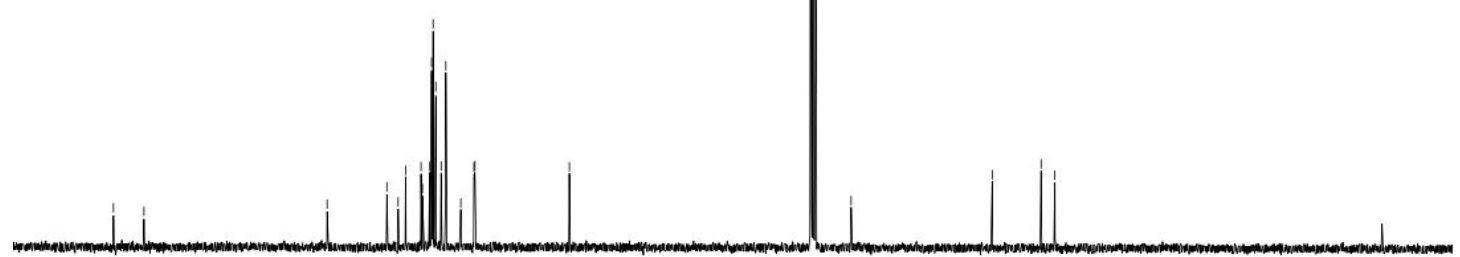

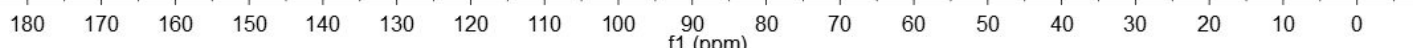

${ }^{1} \mathrm{H}$ and ${ }^{13} \mathrm{C}$ NMR spectra of $\mathbf{6 a b}$ 
$\mathrm{Cl}$

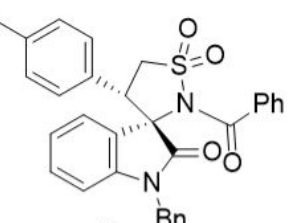

6ac ${ }^{B n}$

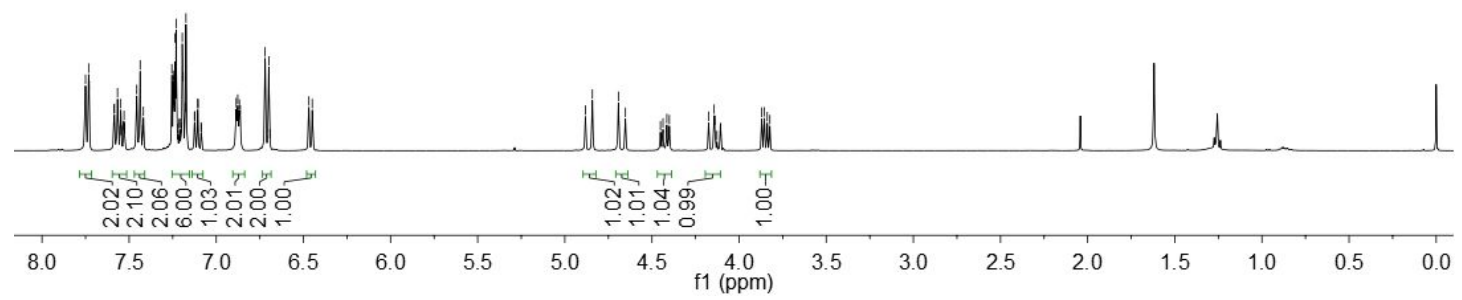

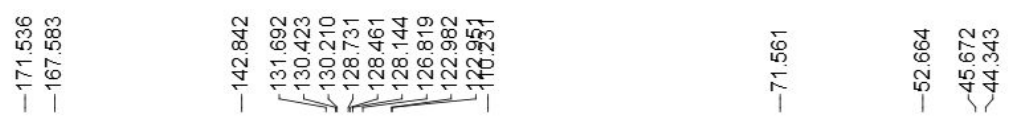
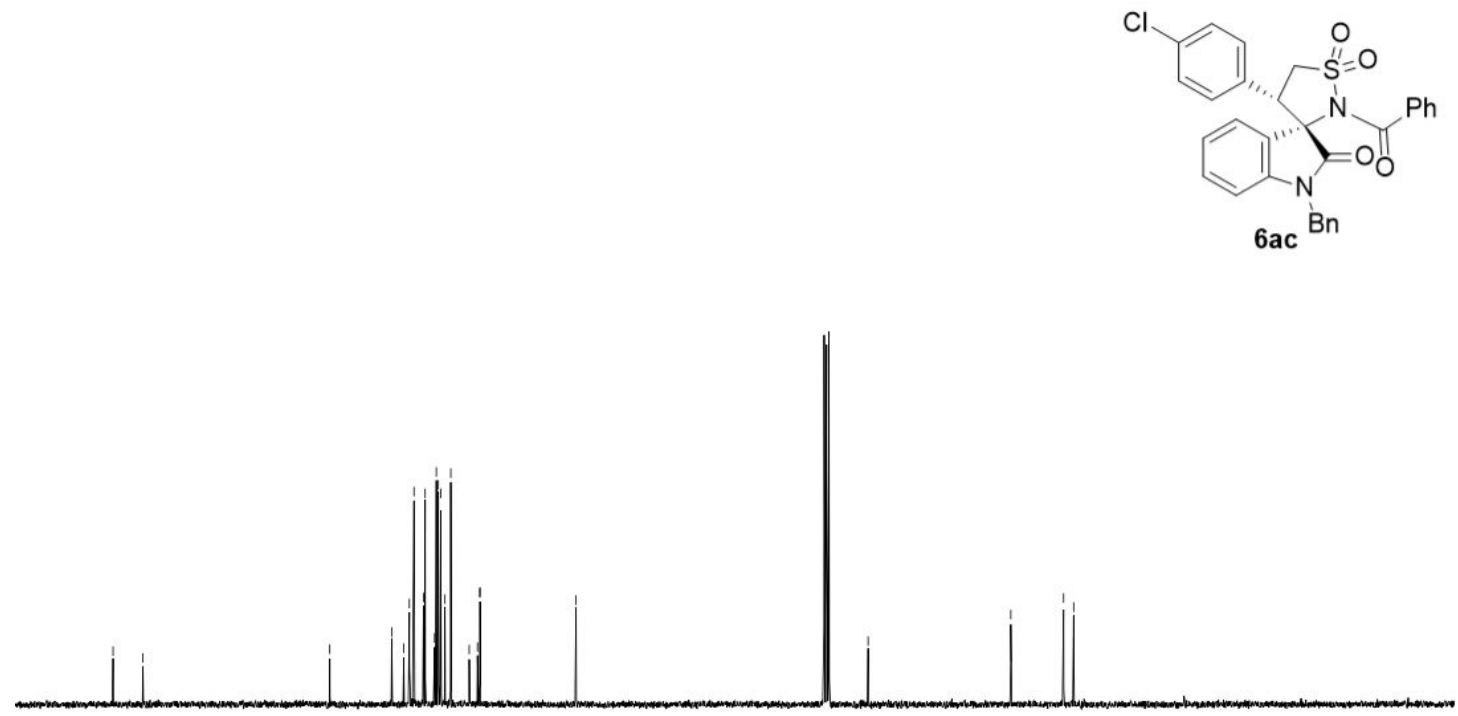

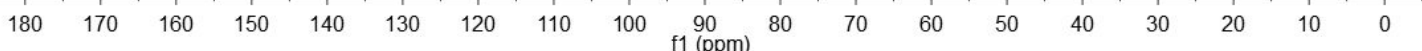

${ }^{1} \mathrm{H}$ and ${ }^{13} \mathrm{C}$ NMR spectra of $\mathbf{6 a c}$ 


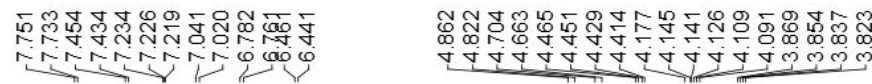
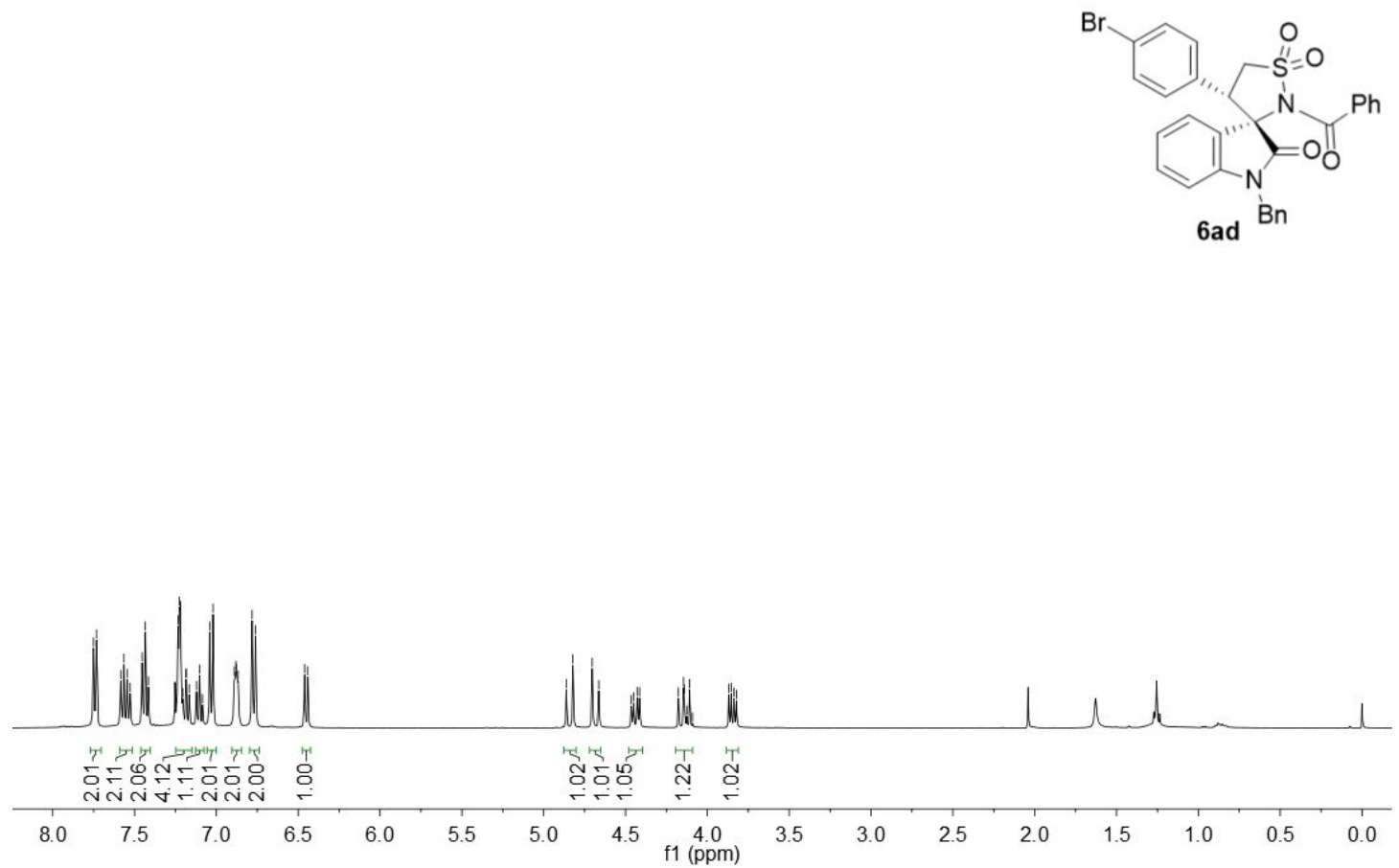

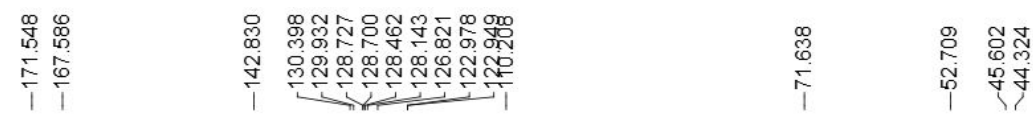

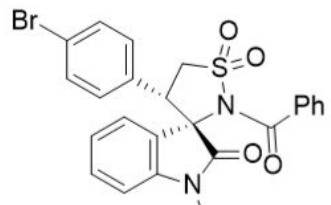

6ad Bn

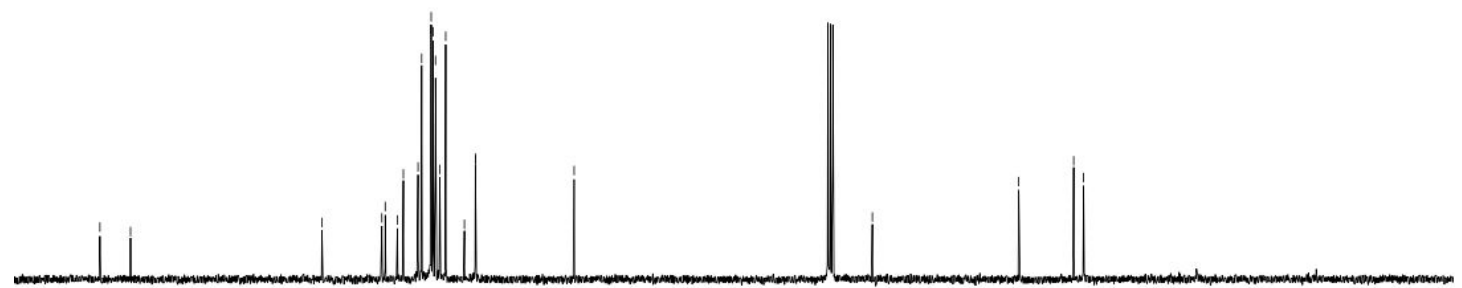

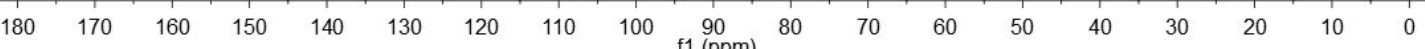

${ }^{1} \mathrm{H}$ and ${ }^{13} \mathrm{C}$ NMR spectra of $\mathbf{6 a d}$ 


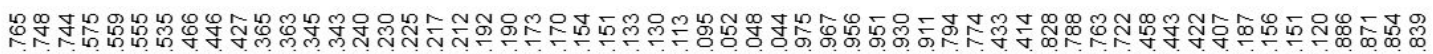

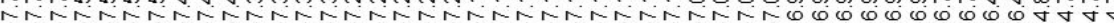<smiles>O=C1c2ccccc2N1NC(=O)C1(c2ccccc2)Cc2ccccc2S1(=O)=O</smiles>

6ae Bn

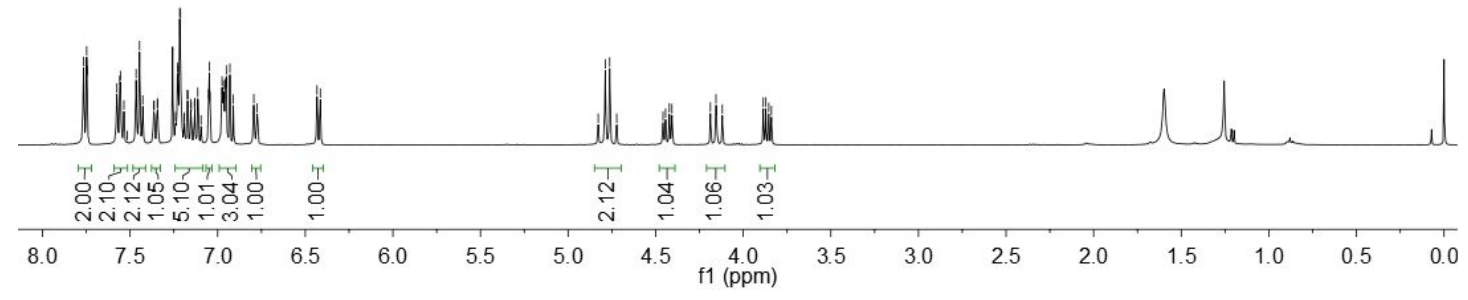
象
$\stackrel{1}{\div}$

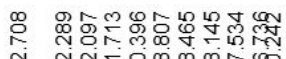
ป

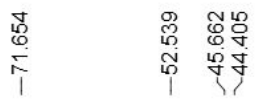

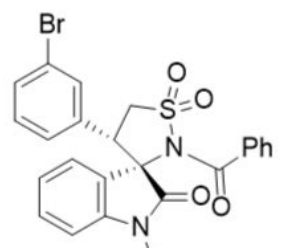

6ae $\mathrm{Bn}$

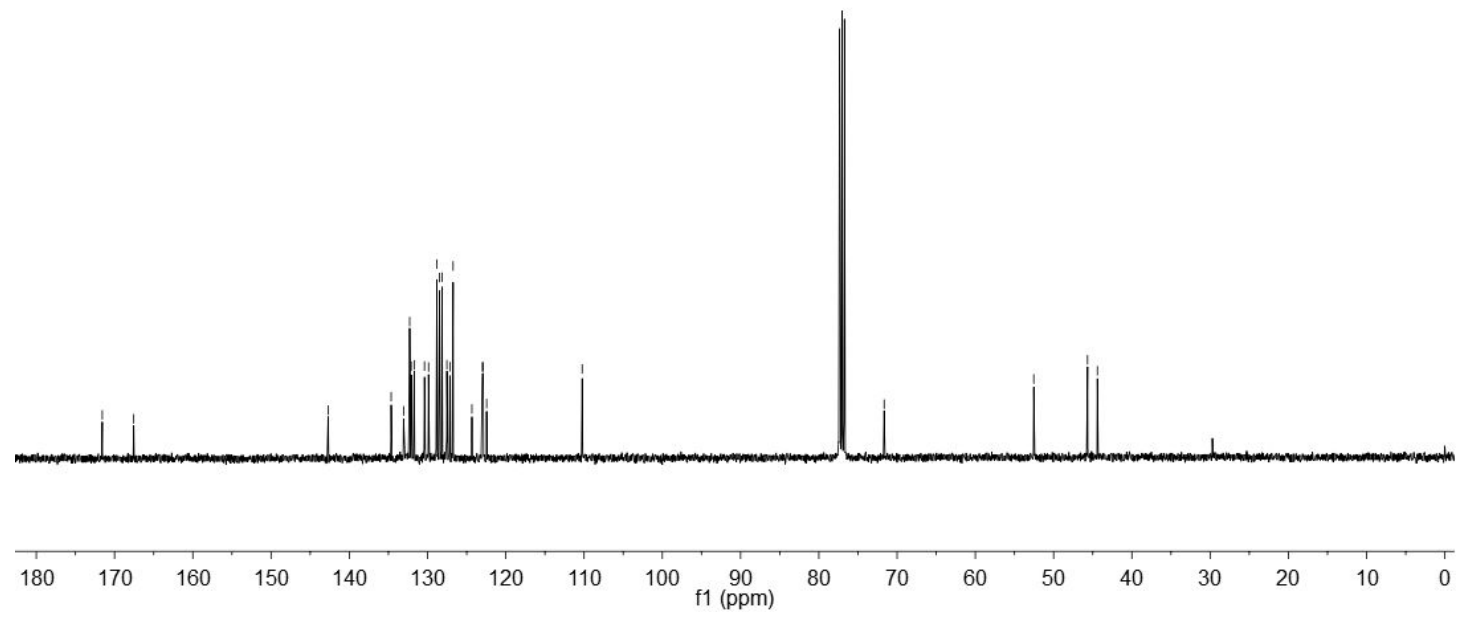

${ }^{1} \mathrm{H}$ and ${ }^{13} \mathrm{C}$ NMR spectra of $6 \mathbf{6 e}$ 


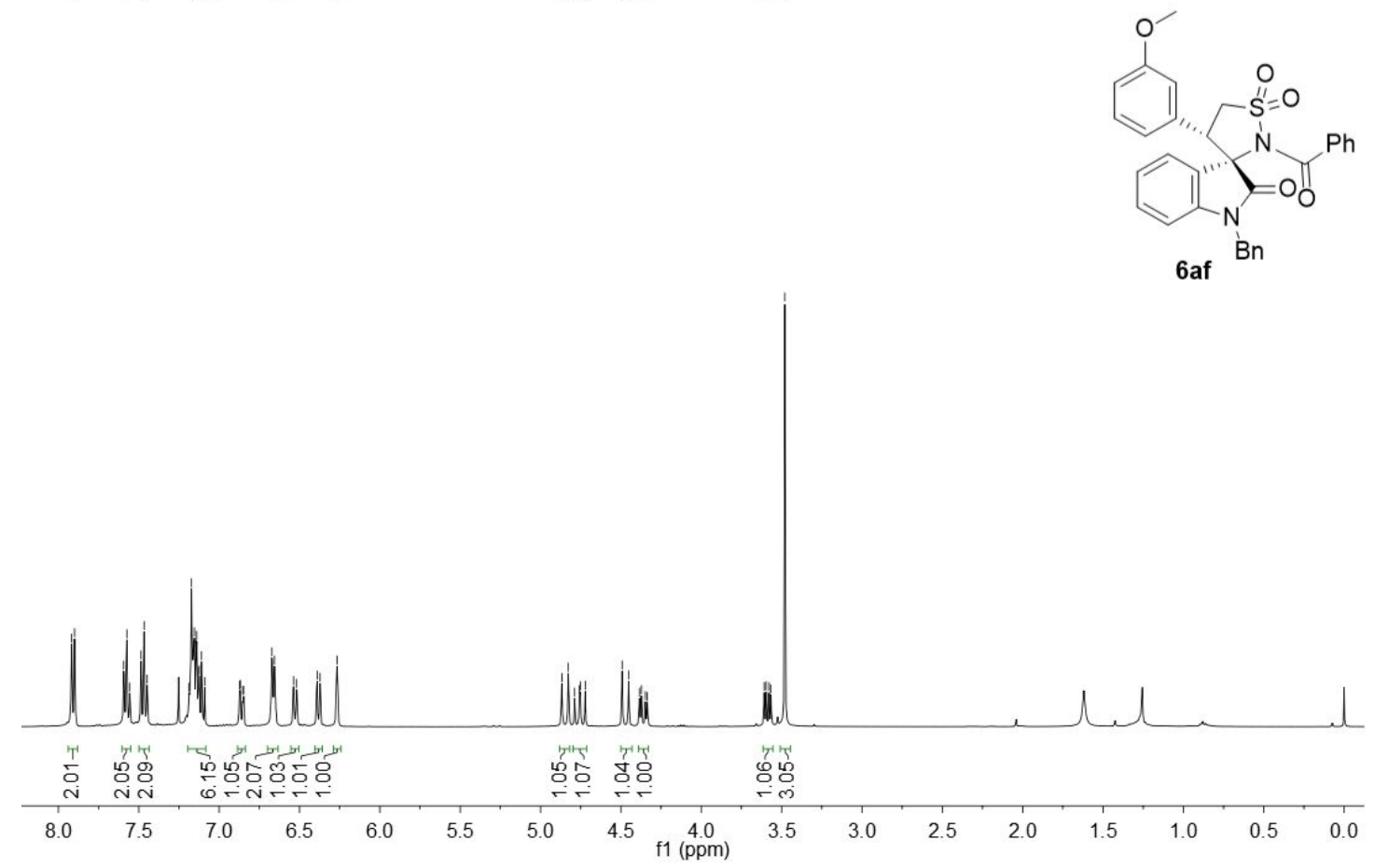

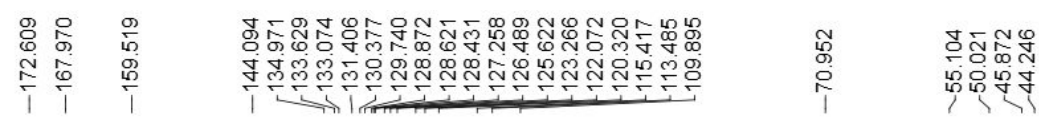
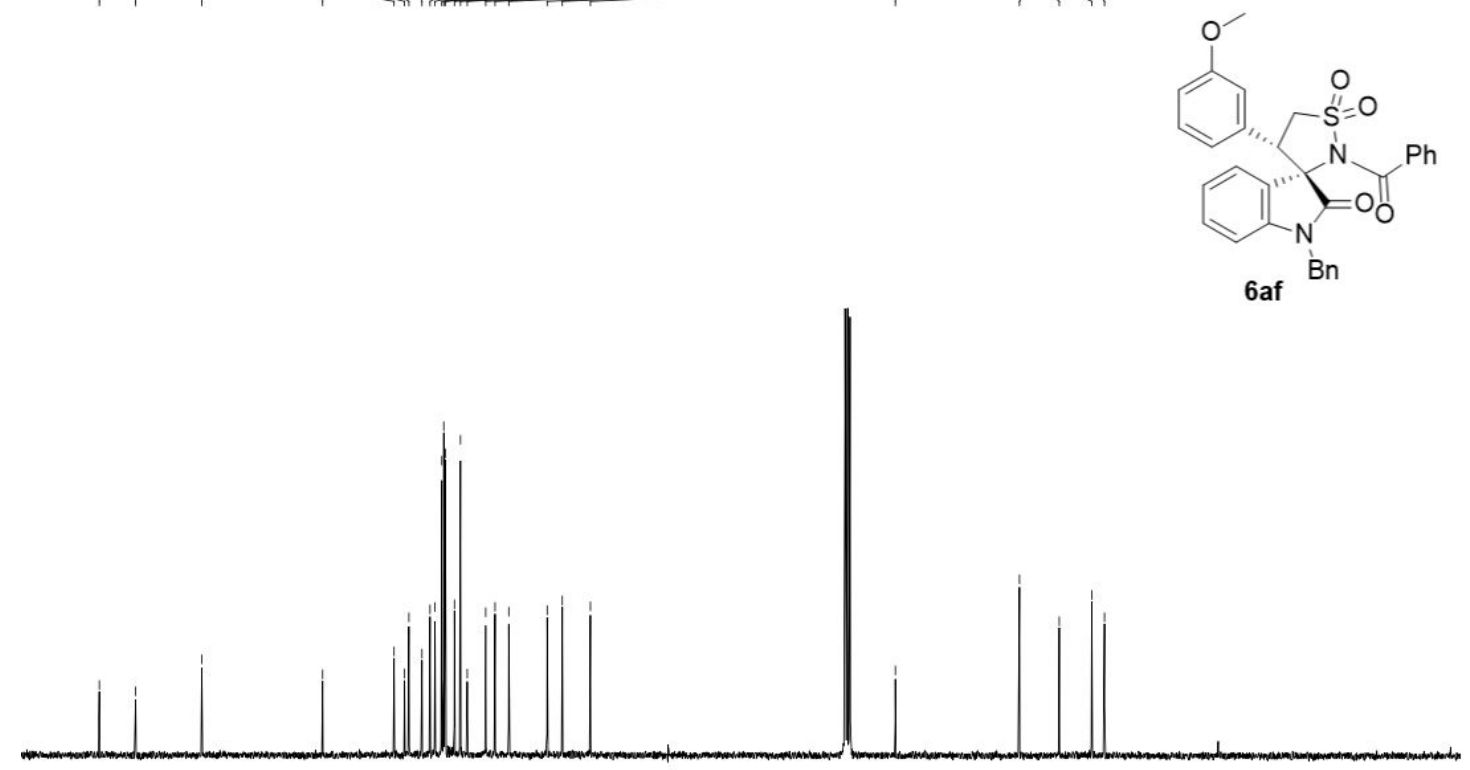

6af $\mathrm{Bn}$

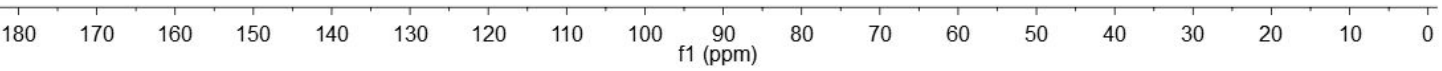

${ }^{1} \mathrm{H}$ and ${ }^{13} \mathrm{C}$ NMR spectra of $\mathbf{6 a f}$ 


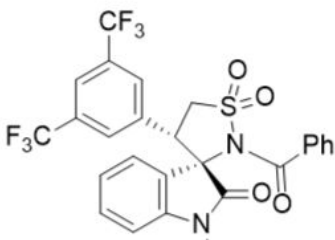

6ag Bn

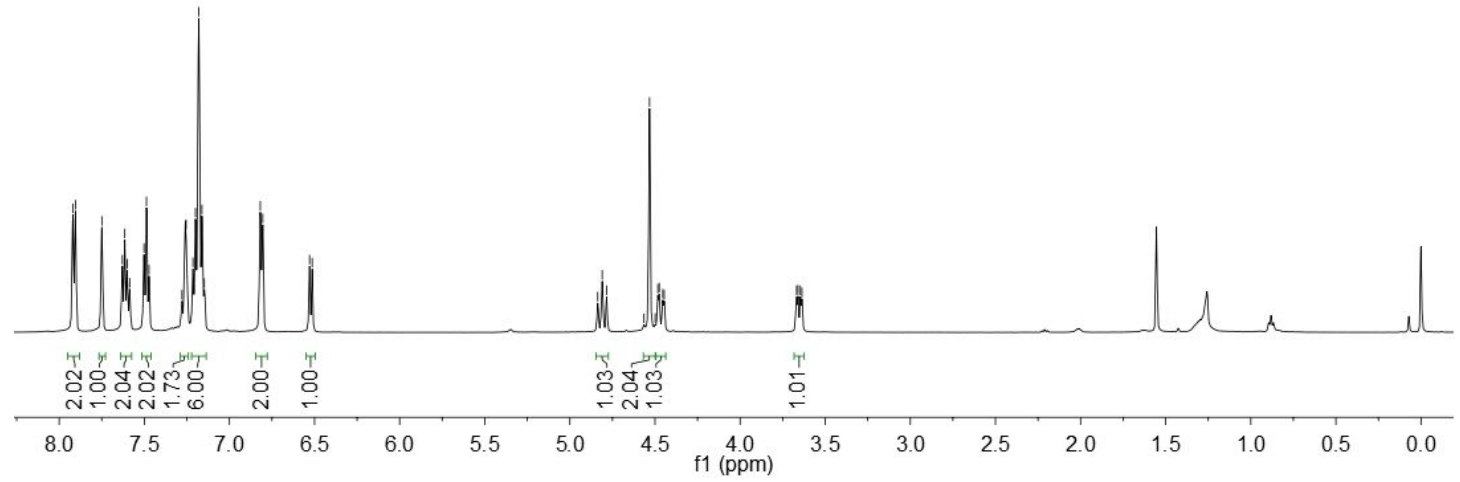

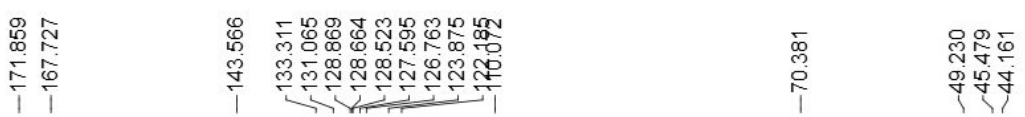

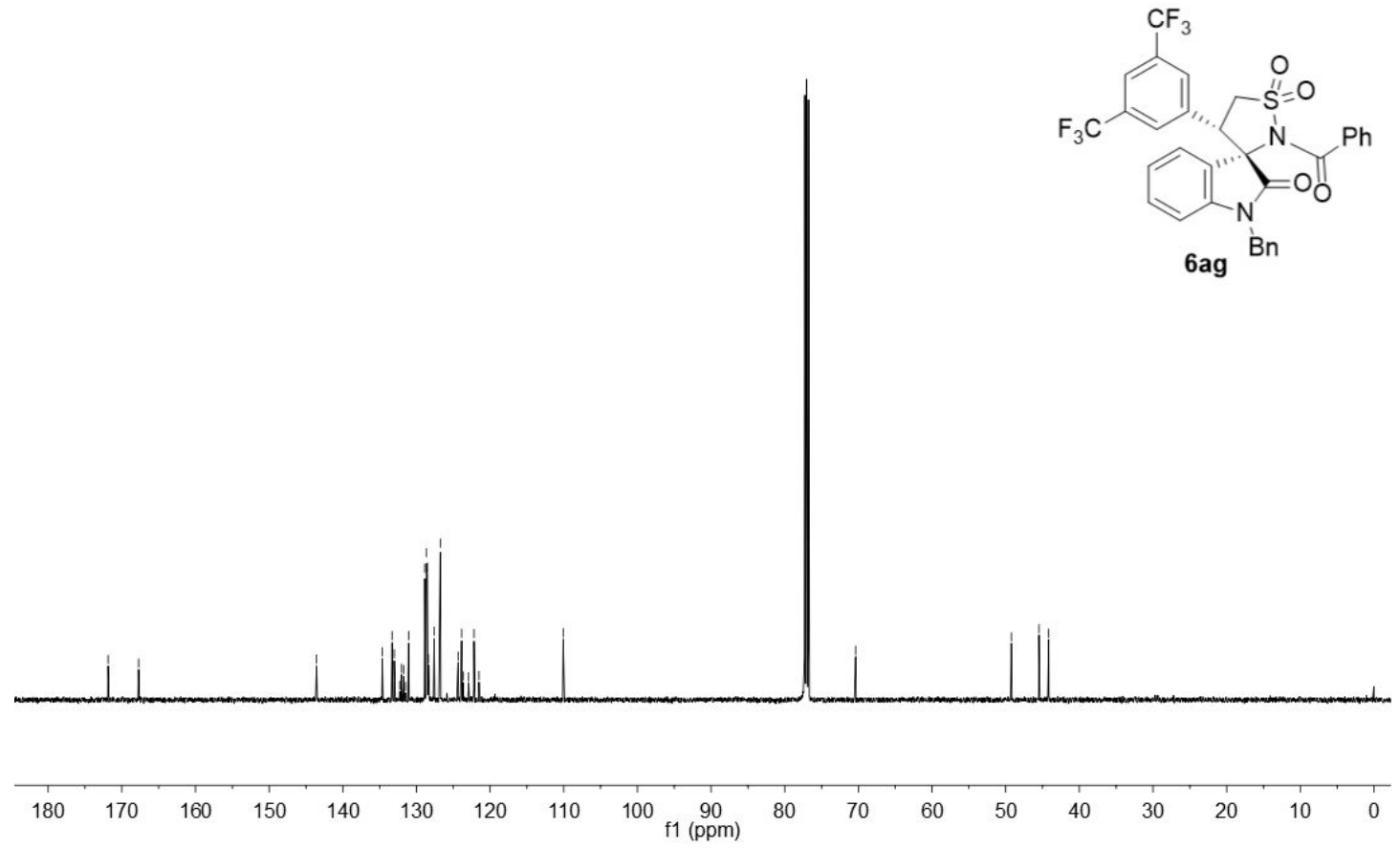

${ }^{1} \mathrm{H}$ and ${ }^{13} \mathrm{C}$ NMR spectra of $\mathbf{6 a g}$ 


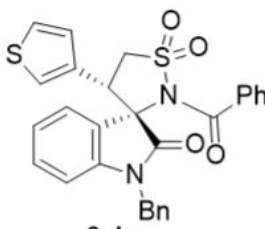

6ah

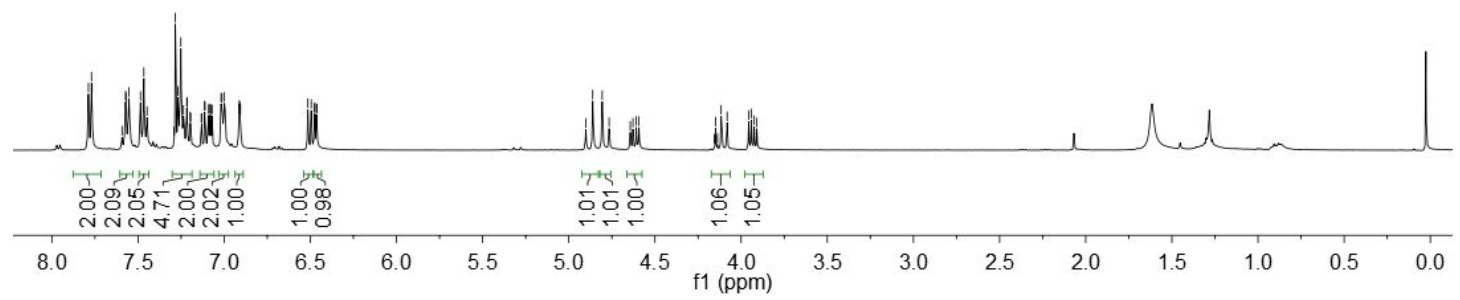

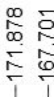
Z
范

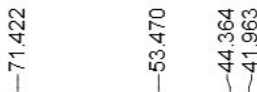

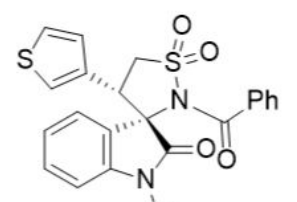

$6 a{ }^{B}$

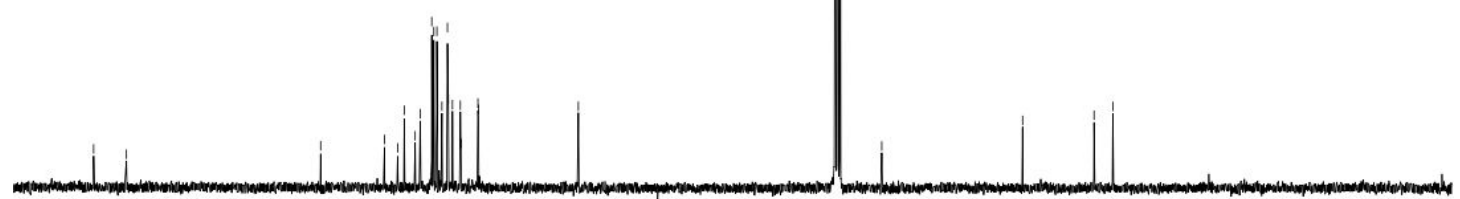

180

$\begin{array}{lllllllll}170 & 160 & 150 & 140 & 130 & 120 & 110 & 100 & \begin{array}{c}90 \\ \mathrm{f} 1(\mathrm{ppm})\end{array}\end{array}$

${ }^{1} \mathrm{H}$ and ${ }^{13} \mathrm{C}$ NMR spectra of $\mathbf{6 a h}$ 


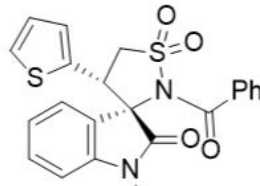

6ai $\mathrm{Bn}$

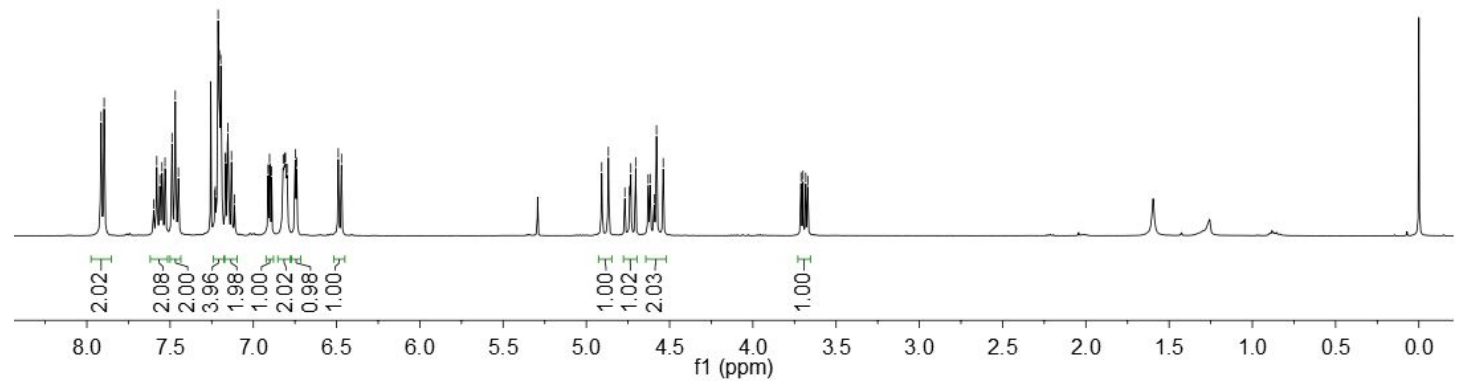

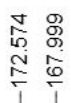

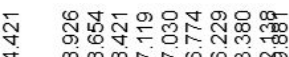

ํํㄴำ

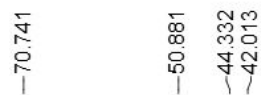

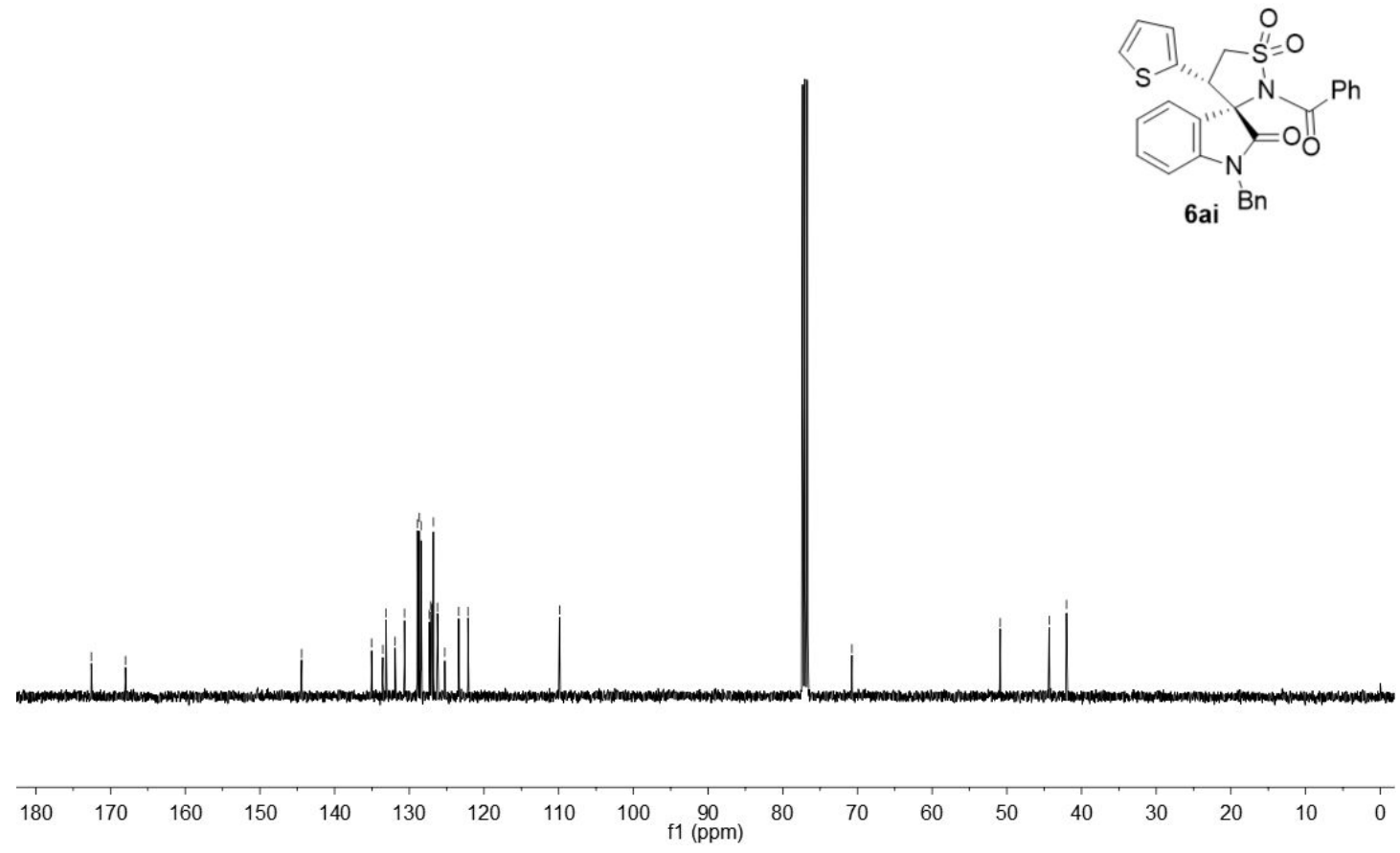

${ }^{1} \mathrm{H}$ and ${ }^{13} \mathrm{C}$ NMR spectra of $\mathbf{6 a i}$ 


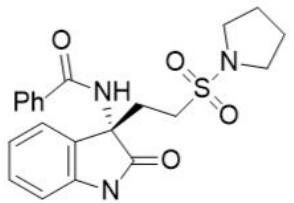

7a Bn

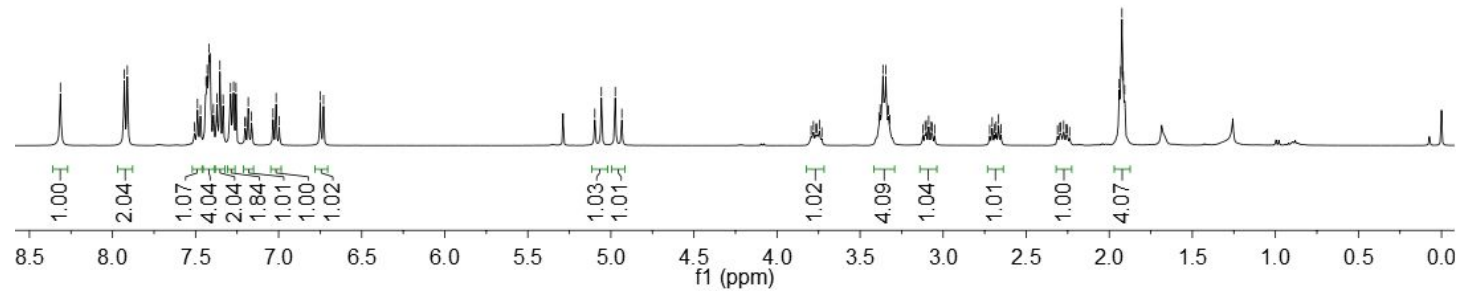

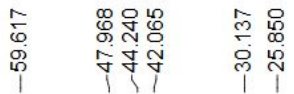

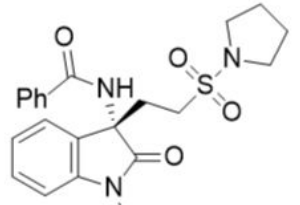

$7 \mathrm{Ban}^{\mathrm{Bn}}$

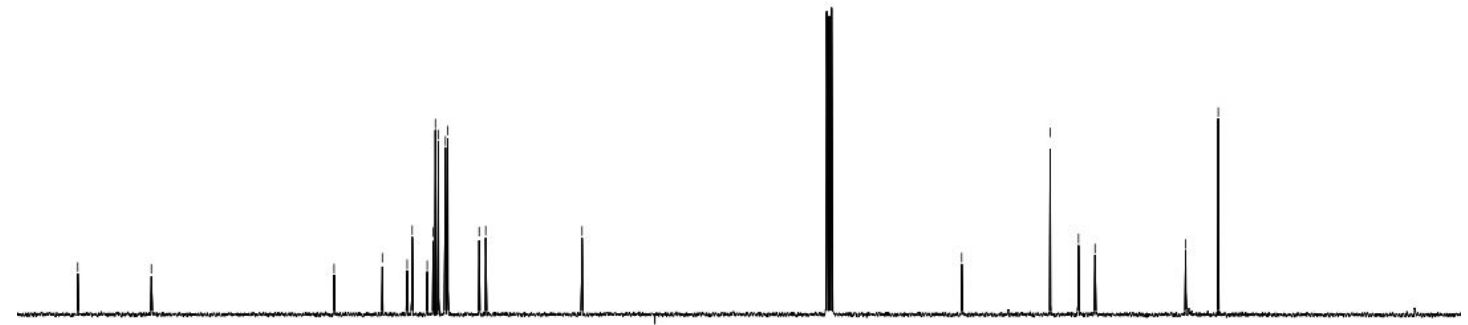

$\begin{array}{lllllllllllllllllll}180 & 170 & 160 & 150 & 140 & 130 & 120 & 110 & 100 & \begin{array}{c}\mathrm{f} 1(\mathrm{ppm}) \\ 80\end{array} & 70 & 60 & 50 & 40 & 30 & 20 & 10 & 0\end{array}$

${ }^{1} \mathrm{H}$ and ${ }^{13} \mathrm{C}$ NMR spectra of $7 \mathbf{a}$ 


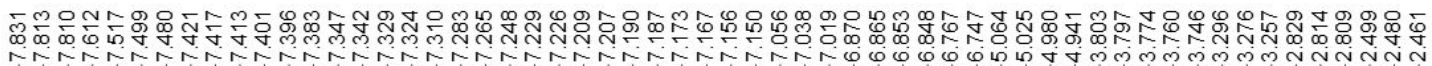

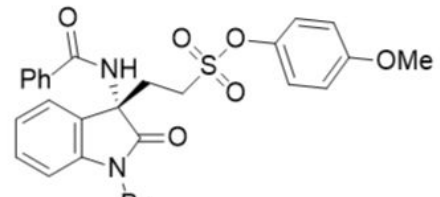

$8 \mathbf{a}$

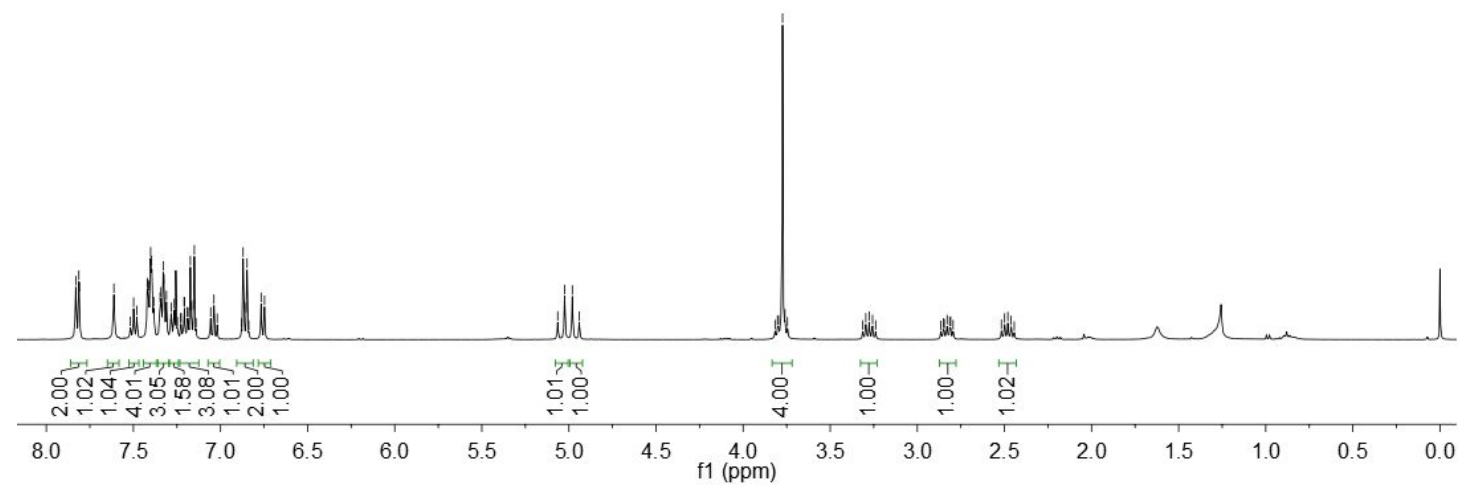

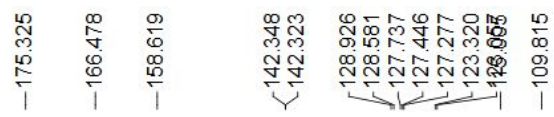

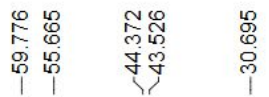

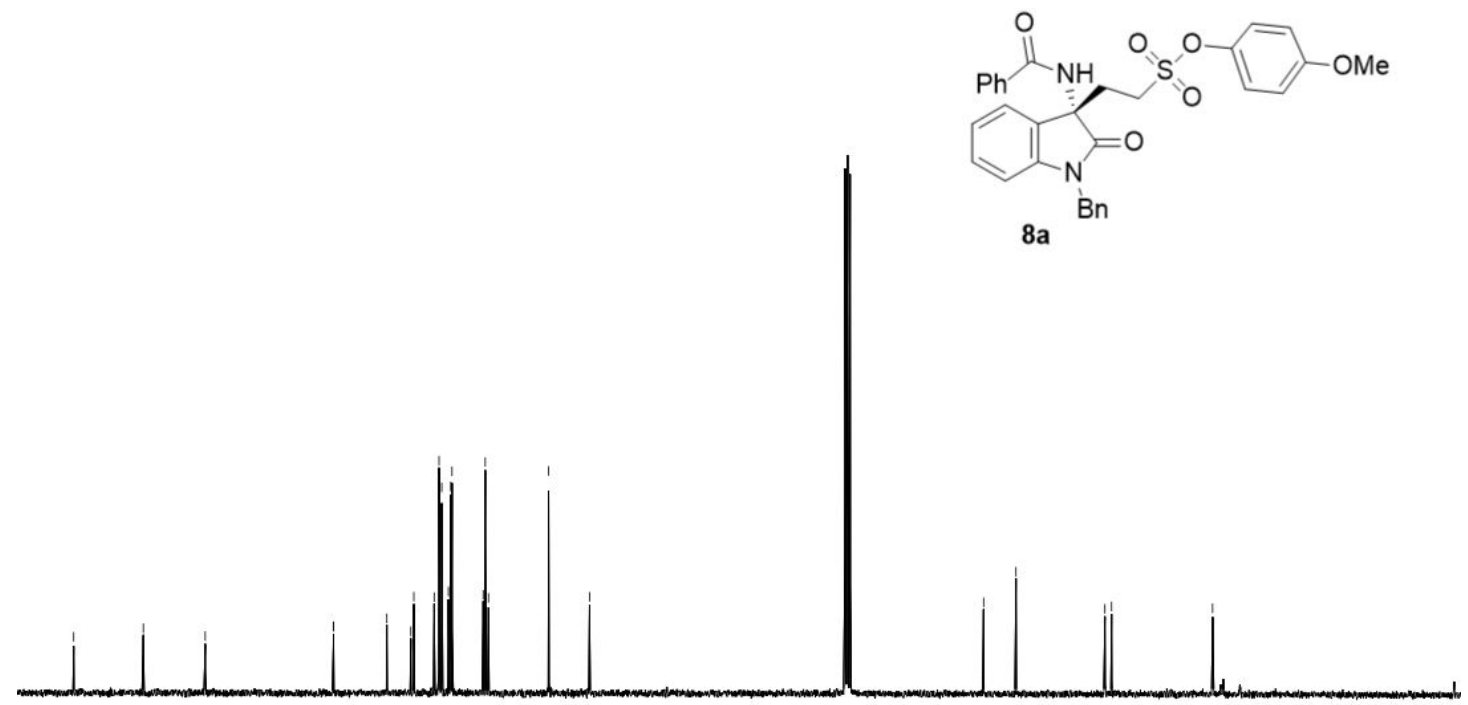

$00 \underset{\mathrm{f} 1(\mathrm{ppm})}{90}$

70

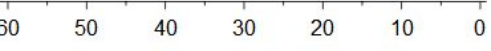

${ }^{1} \mathrm{H}$ and ${ }^{13} \mathrm{C}$ NMR spectra of $\mathbf{8 a}$ 